Magnetic Resonance Imaging of the Prostate

Liang Wang 
ISBN: 978-90-9021799-4

Cover \& Lay-out: Ton Everaers

Illustrations: Liang Wang

Printed by: Print Partners Ipskamp

(c) 2007, L. Wang

Chapter 2, $\odot 2006$ by the Radiological Society of North America

Chapters 3 \& 4, $\odot 2004$ by the Radiological Society of North America

Chapter 5, $\odot 2007$ by the Radiological Society of North America

Chapter 6, ๑ 2007 by the American Roentgen Ray Society

Chapter 7, $\odot 2006$ by the American Roentgen Ray Society

Chapter 8, ๑ 2004 by the American Cancer Society

No part of this book may be reproduced, distributed, stored in a retrievalsystem or transmitted in any form or by any means, without permission of the author, or, when appropriate, of the publishers of the publications. 


\title{
Magnetic Resonance Imaging of the Prostate
}

Beeldvorming van het prostaat met behulp van magnetische resonantie

\author{
Thesis \\ to obtain the degree of Doctor from the \\ Erasmus University Rotterdam \\ by command of the \\ rector magnificus \\ Prof.dr. S.W.J. Lamberts \\ and in accordance with the decision of the Doctorate Board.
}

The public defence shall be held on

Wednesday, 9 May 2007 at 15:45 hrs

by

Liang Wang

born in Lanzhou, China 


\section{Doctoral Committee}

Promotors: Prof. dr. G.P. Krestin

Prof. dr. H. Hricak

Other members: Prof. dr. M.G.M. Hunink

Prof. dr. C.H. Bangma

Prof. dr. J.O. Barentsz

The financial support by the Departments of Radiology of the Erasmus MC - University Medical Center Rotterdam, NL and of the Memorial Sloan-Kettering Cancer Center in New York, NY, USA for the publication of this thesis is gratefully acknowledged. 
To my dear family: father Wang Xinchun, mother Chen Tiejv, wife Qiubai, and son Ming 


\section{CONTENTS}

\section{Chapter 1}

Introduction

\section{Chapter 2}

Prediction of organ-confined prostate cancer: Incremental value of MR imaging and MR spectroscopic imaging to staging nomograms

(Radiology 238:597-603, 2006)

\section{Chapter 3}

Prostate cancer: Incremental value of endorectal MR imaging findings for prediction of extracapsular extension (Radiology 232:133-9, 2004)

\section{Chapter 4}

Prostate cancer: Detection of extracapsular extension by genitourinary and general body radiologists at MR imaging (Radiology 232:140-6, 2004)

\section{Chapter 5}

Prediction of seminal vesicle invasion in prostate cancer: Incremental value of adding endorectal MR imaging to the Kattan nomogram (Radiology 242:182-8, 2007)

\section{Chapter 6}

Incremental value of multiplanar cross-referencing for prostate cancer staging by endorectal MRI (Am J Roentgenology 188:99-104, 2007)

\section{Chapter 7}

Combined endorectal and phased array MRI in the prediction of pelvic lymph node metastasis in prostate cancer (Am J Roentgenology 186:743-8, 2006)

\section{Chapter 8}

The role of preoperative endorectal magnetic resonance imaging in the decision regarding whether to preserve or resect neurovascular bundles during radical retropubic prostatectomy (Cancer 100:2655-63, 2004)

\section{Chapter 9}

The role of MRI in improving pre-operative nomograms to predict biochemical recurrence-free probability in patients undergoing radical prostatectomy (submitted, J Urology) 


\section{Chapter 10}

Assessment of biological aggressiveness of prostate cancer: Correlation of MR signal intensity with Gleason grade on whole-mount step-section pathologic analysis after radical prostatectomy (accepted for publication in Radiology)

\section{Chapter 11}

Post processing of dynamic gadolinium-enhanced MRI exams of the liver:

Explanation and potential clinical applications for color-coded qualitative and quantitative analysis(accepted for publication in Acta Radiologica)

\section{Chapter 12}

\section{Chapter 14}

\section{Chapter 15}

\section{Chapter 16}

\section{Chapter 17}

Publications

\section{Chapter 18}


Criphestroduction 


\section{INTRODUCTION}

$\mathbf{P}$

rostate cancer is a major public health problem as well as an economic burden in the United States and European Union (1-4). Currently, prostate cancer is the most common non-cutaneous cancer and the third leading cause of cancer death in men in the United State and European Union (1, 4). An estimated 234,460 new cases of prostate cancer will be diagnosed in the United States and 27,350 people will die of the disease in 2006 (1). In the next decades the increasing natural life expectation will result in a further increase of both the incidence of and the related deaths from prostate cancer $(1,5)$.

The high incidence and protracted natural history of prostate cancer, combined with earlier detection, and an increasing proportion of patients with smaller-volume and lower-grade lesions at diagnosis, have complicated the management of the disease and raised controversial issues regarding screening, selection of patients for treatment, and superiority among treatment modalities $(1,6-8)$. The changes due to prostate-specific antigen (PSA) screening have led to an unfortunate trend of overdetection and overtreatment of biologically insignificant disease $(9,10)$. One challenge that clinicians and patients have to face in the modern PSA era is to differentiate men who have disease destined to progress and cause morbidity/ mortality from those who will not require immediate, or possibly even delayed, therapeutic intervention (11-14).

Patients with prostate cancer have to choose from a wide range of treatments, and the decision significantly affects both quality of life (QOL) and survival. Primary therapies with curative intent (surgery or radiation) provide excellent long-term cancer control but are accompanied by a risk of treatment-related morbidity $(15,16)$.There is a growing demand for patient-specific therapies that can reduce treatment morbidity while maximizing treatment benefits. An objective prior to any cancer therapy is to obtain a comprehensive and accurate knowledge of tumor location, size, locoregional extent, and biologic potential. Better tools are needed to help physicians and patients decide what type of treatment is most appropriate, or whether any treatment is needed at all.

Scientific discoveries, technologic advances and research efforts are profoundly changing our understanding of prostate cancer and our ability to treat it effectively (5). The Nobel prize-winning discoveries of Paul C. Lauterbur (17) and Sir Peter Mansfield (18) concerning magnetic resonance imaging (MRI) and Richard R. Ernst (19) concerning the development of the methodology of high resolution nuclear magnetic resonance spectroscopic imaging (MRSI) led to the development of modern MR imaging to take pictures of anatomical structures and MR spectroscopy to measure amounts of important chemicals within the body noninvasively - a breakthrough in medical diagnostics and research. Historically, in 1984 Hricak, et al used MR imaging as a local staging modality for prostate cancer (20, 21). In 1991 picture archiving and communication systems (PACS) started to be available for clinical use (22). PACS is a collection of technologies used to facilitate digital medical imaging display and distribution. In 1995 Krestin, et al demonstrated the use of contrast-enhanced endorectal coil MRI in local staging of prostate cancer (23). In 1995, Hricak, et al showed integration of endorectal-pelvic phased-array coils for MRI of prostate cancer $(24,25)$. In 1996 Kurhanewicz, et al reported that combined endorectal MRI/MRSI could be considered a "one-stop shop" imaging modality for assessing anatomic and metabolic features of prostate cancer (26). In 2002 Harisinghani et al. showed that high-resolution MRI with lymphotropic superparamagnetic nanoparticles to detect small and otherwise undetectable lymph node metastasis (LNM) in prostate cancer (27).

Although reports on the value of MRI in prostate cancer management have been contradictory, there is no doubt that the use of endorectal MRI and MRSI for the noninvasive evaluation of tumor location, local extent [extracapsular extension (ECE) and/or seminal vesicle invasion (SVI)], volume, and aggressiveness is generating strong clinical interest $(6,28-34)$. Advances in prostate MRI have improved the pre-treatment characterization of prostate cancer (35, 36). In addition to patient selection, MRI may aid in directing therapy, evaluating results, and monitoring for early recognition of treatment failure, leading to the best cancer-control and QOL outcomes $(6,37)$.

The central subject of this dissertation is the ability of state-of-the-art MRI/MRSI to non-invasively improve staging, treatment planning, and the assessment of tumor aggressiveness and recurrence-free probabilities in patients with prostate cancer. The dissertation also explores the potential clinical application of color-coded parameter imaging derived from postprocessing of dynamic multiphasic gadolinium-enhanced MRI (DMGE-MRI) of abdominal tumors. 


\section{OUtLINE OF THE DISSERTATION}

Chapter $\mathbf{2}$ of the dissertation describes the incremental value of endorectal coil MR imaging and combined endorectal MRI/MRSI to the staging nomograms for predicting organ-confined prostate cancer (OCPC). Chapter $\mathbf{3}$ discusses a study showing that endorectal MR imaging contributes significant incremental value to clinical variables in the prediction of ECE. Chapter $\mathbf{4}$ shows that the predictive value of endorectal MR imaging findings in the detection of ECE is significantly affected by the reader's subspecialty experience. Intraobserver variability, as well as inconsistency in the accuracy of diagnoses determined with MR imaging findings, has been reported. Chapter $\mathbf{5}$ discusses a study in a large, contemporary cohort of patients that showed that endorectal MRI findings contribute incremental value to the Kattan nomogram for the prediction of prostate cancer SVI. Chapter $\mathbf{6}$ describes a study showing that the cross-referencing tool on PACS improves prostate cancer tumor staging by three dimensional (3D) endorectal MRI. Chapter $\mathbf{7}$ presents an investigation of the role of combined endorectal and phased-array MRI in the detection of lymph node metastasis (LNM) in a representative sample of the current prostate cancer patient population, with its low incidence of LNM. The prospective study described in Chapter $\mathbf{8}$ shows that the use of endorectal MRI before radical prostatectomy (RP) could help the surgeon make a more appropriate management decision regarding whether to spare or resect the neurovascular bundles during RP. The surgeon's ultimate goal is to excise the cancer completely while preserving the nearby normal structures, thus avoiding positive surgical margin and minimizing the chances of recurrence and allowing recovery of sexual function and avoidance of impotence (erectile dysfunction). Chapter 9 investigates whether the addition of endorectal MRI findings to an established preoperative nomogram would improve prediction of freedom from biochemical recurrence. Chapter $\mathbf{1 0}$ shows that signal intensity evaluation of T2-weighted MR images may facilitate noninvasive assessment of prostate cancer biological aggressiveness. Chapter 11 illustrates the potential clinical application of color-coded parameter imaging derived from postprocessing of dynamic multiphasic gadolinium-enhanced MRI (DMGE-MRI) of abdominal tumors. In Chapter 12 the data presented in the preceding chapters are discussed as a whole and prospects and recommendations regarding diagnostic MRI of prostate cancer are given.

\section{REFERENCES:}

1. Jemal A, Siegel R, Ward E, et al. Cancer statistics, 2006. CA Cancer J Clin 2006; 56:106-130.

2. Parkin DM, Bray F, Ferlay J, Pisani P. Global cancer statistics, 2002. CA Cancer J Clin 2005; 55:74-108.

3. Kamangar F, Dores GM, Anderson WF. Patterns of cancer incidence, mortality, and prevalence across five continents: defining priorities to reduce cancer disparities in different geographic regions of the world. J Clin Oncol 2006; 24:2137-2150.

4. Boyle P, Ferlay J. Cancer incidence and mortality in Europe, 2004. Ann Oncol 2005; 16:481-488.

5. Scardino PT. New technology and the changing world of cancer. Nat Clin Pract Urol 2005; 2:403.

6. Hricak H. MR imaging and MR spectroscopic imaging in the pre-treatment evaluation of prostate cancer. Br J Radiol 2005; 78 Spec No 2: S103-111.

7. Stamey TA, Caldwell M, McNeal JE, Nolley R, Hemenez M, Downs J. The prostate specific antigen era in the United States is over for prostate cancer: what happened in the last 20 years? J Urol 2004; 172:1297-1301.

8. Galper SL, Chen MH, Catalona WJ, Roehl KA, Richie JP, D'Amico AV. Evidence to support a continued stage migration and decrease in prostate cancer specific mortality. J Urol 2006; 175:907-912.

9. Carroll PR. Early stage prostate cancer--do we have a problem with over-detection, overtreatment or both? J Urol 2005; 173:1061-1062.

10. Cooperberg MR, Lubeck DP, Meng MV, Mehta SS, Carroll PR. The changing face of low-risk prostate cancer: trends in clinical presentation and primary management. J Clin Oncol 2004; 22:2141-2149.

11. Freedland SJ, Partin AW. Prostate-specific antigen: update 2006. Urology 2006; 67:458-460.

12. Schroder FH. Prostate specific antigen and other markers for prostate cancer. J Urol 2006; 175:1199-1200.

13. Lieberman R. Evidence-based medical perspectives: the evolving role of PSA for early detection, monitoring of treatment response, and as a surrogate end point of efficacy for interventions in men with different clinical risk states for the prevention and progression of prostate cancer. Am J Ther 2004; 11:501-506.

14. Hricak H, Schoder H, Pucar D, et al. Advances in imaging in the postoperative patient with a rising prostate-specific antigen level. Semin Oncol 2003; 30:616-634

15. Saranchuk JW, Kattan MW, Elkin E, Touijer AK, Scardino PT, Eastham JA. Achieving optimal outcomes after radical prostatectomy. J Clin Oncol 2005; 23:4146-4151

16. Bianco FJ, Jr., Scardino PT, Eastham JA. Radical prostatectomy: long-term cancer control and recovery of sexual and urinary function ("trifecta"). Urology 2005; 66:83-94.

17. Lauterbur PC. Nobel Lecture. All science is interdisciplinary--from magnetic moments to molecules to men. Biosci Rep 2004; 24:165-178.

18. Mansfield P. Snapshot magnetic resonance imaging (Nobel lecture). Angew Chem Int Ed Engl 2004; 43:5456-5464.

19. Ernst RR. Nobel Lecture. Nuclear magnetic resonance Fourier transform spectroscopy. Biosci Rep 1992; 12:143-187.

20. Hricak H, Williams RD. Magnetic resonance imaging and its application in urology. Urology 1984; 23:442-454.

21. Williams RD, Hricak H. Magnetic resonance imaging in urology. J Urol 1984; 132:641-649.

22. Miyamoto K, Abe S, Kawakami Y. Picture archiving and communication system in Hokkaido University Hospital: advantage and disadvantage of HU-PACS chest roentgenogram images in the outpatient clinic. J Digit Imaging 1991;4:28-31. 
23. Huch Boni RA, Boner JA, Lutolf UM, Trinkler F, Pestalozzi DM, Krestin GP. Contrast-enhanced endorectal coil MRI in local staging of prostate carcinoma. J Comput Assist Tomogr 1995; 19:232-237.

24. Hricak H, White S, Vigneron D, et al. Carcinoma of the prostate gland: MR imaging with pelvic phased-array coils versus integrated endorectal--pelvic phased-array coils. Radiology 1994; 193:703-709.

25. Hricak H. Given the improvement in pelvic coils for MR, is an endorectal coil necessary to evaluate prostate carcinoma? AJR Am J Roentgenol 1995; 165:733-734.

26. Kurhanewicz J, Vigneron DB, Hricak H, Narayan P, Carroll P, Nelson SJ. Three-dimensional H-1 MR spectroscopic imaging of the in situ human prostate with high (0.24-0.7-cm3) spatial resolution. Radiology 1996; 198:795-805.

27. Harisinghani MG, Barentsz JO, Hahn PF, et al. MR lymphangiography for detection of minimal nodal disease in patients with prostate cancer. Acad Radiol 2002; 9 Suppl 2:S312-313.

28. Trabulsi EJ, Merriam WG, Gomella LG. New imaging techniques in prostate cancer. Curr Urol Rep 2006; 7:175-180.

29. Wang L, Hricak H, Kattan MW, Chen HN, Scardino PT, Kuroiwa K. Prediction of organ-confined prostate cancer: incremental value of MR imaging and MR spectroscopic imaging to staging nomograms. Radiology 2006; 238:597-603.

30. Coakley FV, Qayyum A, Kurhanewicz J. Magnetic resonance imaging and spectroscopic imaging of prostate cancer. J Urol 2003; 170:S6975; discussion S75-66.

31. Wang L, Mullerad M, Chen HN, et al. Prostate cancer: incremental value of endorectal MR imaging findings for prediction of extracapsular extension. Radiology 2004; 232:133-139.

32. Sala E, Akin O, Moskowitz CS, et al. Endorectal MR imaging in the evaluation of seminal vesicle invasion: diagnostic accuracy and multivariate feature analysis. Radiology 2006; 238:929-937.

33. Jager GJ, Severens JL, Thornbury JR, de La Rosette JJ, Ruijs SH, Barentsz JO. Prostate cancer staging: should MR imaging be used?--A decision analytic approach. Radiology 2000; 215:445-451.

34. Engelbrecht MR, Jager GJ, Laheij RJ, Verbeek AL, van Lier HJ, Barentsz JO. Local staging of prostate cancer using magnetic resonance imaging: a meta-analysis. Eur Radiol 2002; 12:2294-2302.

35. Rajesh A, Coakley FV. MR imaging and MR spectroscopic imaging of prostate cancer. Magn Reson Imaging Clin N Am 2004; 12:557-579, vii.

36. van Dorsten FA, van der Graaf M, Engelbrecht MR, et al. Combined quantitative dynamic contrast-enhanced MR imaging and (1)H MR spectroscopic imaging of human prostate cancer. J Magn Reson Imaging 2004; 20:279-287.

37. Huzjan R, Sala E, Hricak H. Magnetic resonance imaging and magnetic resonance spectroscopic imaging of prostate cancer. Nat Clin Pract Urol 2005; 2:434-442. 
1 
Prediftion of Organ-Confined Prostate Cancer: Incremental Value of MR Imaging and MR Spectroscopic Imaging to Staging Nomograms 


\title{
Prediction of Organ-Confined Prostate Cancer: Incremental Value of MR Imaging and MR Spectroscopic Imaging to Staging Nomograms
}

\author{
Supported by National Institutes of Health grant R01 CA7642
}

Liang Wang, Hedvig Hricak, Michael W. Kattan, Hui-Ni Chen, Peter T. Scardino and Kentaro Kuroiwa

From the Departments of Radiology (L.W., H.H., H.N.C.) and Urology (M.W.K., P.T.S., K.K.), Memorial SloanKettering Cancer Center, 1275 York Ave, New York, NY 10021. From the 2004 RSNA Annual Meeting. Received November 10, 2004; revision requested December 16; revision received May 9, 2005; accepted May 19; final version accepted June 17.

\section{Abstract}

Purpose: To assess retrospectively the incremental value of endorectal coil magnetic resonance (MR) imaging and combined endorectal MR imaging-MR spectroscopic imaging to the staging nomograms for predicting organconfined prostate cancer (OCPC).

Materials and Methods: The institutional review board approved this HIPAA-compliant study and issued a waiver of informed consent for review of the MR reports and clinical data. Between November 1, 1999, and November 1, 2004, 229 patients underwent endorectal MR imaging and 383 underwent combined endorectal MR imaging-MR spectroscopic imaging before radical prostatectomy. Mean patient age was 58 years (range, 32-74 years). MR studies were interpreted prospectively by 12 radiologists who were informed of patients' clinical data. On the basis of the MR reports, the risks of extracapsular extension, seminal vesicle invasion, and lymph node metastasis were scored retrospectively from 1 to 5; the highest score was subtracted from 6 to determine a score (from 1 to 5 ) for the likelihood of OCPC on MR studies. The staging nomograms were used to calculate the likelihood of OCPC on the basis of serum prostate-specific antigen level, Gleason grade at biopsy, and clinical stage. Histopathologic findings constituted the reference standard. Logistic regression was used to estimate the multivariable relations between OCPC and MR findings. The area under the receiver operator characteristic curve was calculated for each model. The jackknife method was used for bias correction.

Results: MR findings contributed significant incremental value $(P \leq .02)$ to the nomograms in the overall study population. The contribution of MR findings was significant in all risk groups but was greatest in the intermediate- and high-risk groups ( $P<.01$ for both). Accuracy in the prediction of OCPC with MR was higher when MR spectroscopic imaging was used, but the difference was not significant.

Conclusion: Endorectal MR imaging and combined endorectal MR imaging-MR spectroscopic imaging contribute significant incremental value to the staging nomograms in predicting OCPC.

() RSNA, 2005 


\section{INTRODUCTION}

$\mathbf{T}$ oday, with the widespread use of screening of prostate-specific antigen (PSA)' levels and with better methods for diagnosis at biopsy, $90 \%$ of newly diagnosed cases of prostate cancer are considered to be of a local or regional stage (1). When clinically localized cancers are confined to the prostate pathologically, more than $85 \%$ are curable with radical prostatectomy $(2,3)$.

Effective treatment planning, whether for radiation treatment or surgery, requires accurate prediction of the pathologic stage of the cancer. Various methods have been proposed for predicting that a clinically localized prostate cancer is, in fact, pathologically confined to the prostate (4-8). An important advance in accurate prediction was the development of nomograms that combined clinical stage (determined by means of digital rectal examination), serum PSA levels, and the Gleason grade in the biopsy specimen to predict the pathologic stage of the cancer $(9,10)$. These "Partin Tables" are a validated predictive tool widely used for patient counseling (11-13). However, as a treatment-planning tool they are limited because they do not incorporate anatomic data that could guide interventions to control local disease. If cancer extends outside the prostate, the chances of cure are substantially diminished, and the surgical or radiation treatment planning must be adapted to ensure complete eradication of the cancer. The role of endorectal magnetic resonance (MR) imaging in prostate cancer management has been emerging with improved MR techniques, such as MR spectroscopic imaging, and with better interpretation of MR images of the prostate by experienced radiologists (14-19). The present study was designed to assess retrospectively the incremental value of endorectal coil MR imaging and combined endorectal MR imaging-MR spectroscopic imaging to the staging nomograms for predicting organ-confined prostate cancer (OCPC).

\section{Materials and Methods}

\section{Patients}

Between November 1, 1999, and November 1, 2004, 612 consecutive patients with prostate cancer were referred from the urology department (P.T.S.) for MR imaging before radical retropubic prostatectomy and pelvic lymphadenectomy. Three hundred eighty-three patients underwent endorectal MR imaging combined with MR spectroscopic imaging, and 229 underwent endorectal MR imaging alone. From November 1999 through June 2003, imaging was performed as part of an ongoing National Institutes of Health study investigating the use of MR imaging in patients with prostate cancer; all patients gave informed consent before enrollment in the prospective National Institutes of Health study, which was compliant with the Health Insurance Portability and Accountability Act, or HIPAA. Subsequent to June 2003, imaging was performed as part of our accepted practice for patient evaluation. Our institutional review board issued a waiver of informed consent for the review of the MR reports and clinical data for this retrospective study, which was also HIPAA compliant. Mean patient age was 58 years (range, 32-74 years). None of the patients received neoadjuvant hormonal or radiation therapy prior to surgery. A tissue diagnosis of prostate cancer was made at biopsy in all patients. Clinical stage (determined by means of digital rectal examination), serum PSA level, and Gleason grade in the biopsy specimen, as well as MR data, were recorded retrospectively from the patients' medical records by two coauthors (L.W. and K.K.). A segment of the study population (371 of 612 patients) has been included in previous publications $(14,16,17)$.

\footnotetext{
${ }^{1}$ Abbreviations

$\mathrm{ECE}=$ extracapsular extension $\cdot \mathrm{LNM}=$ lymph node metastasis $\cdot \mathrm{OCPC}=$ organ-confined prostate cancer $\cdot \mathrm{PSA}=$ prostate-specific antigen $\cdot \mathrm{ROC}=$ receiver operating characteristic $\cdot S \mathrm{VI}=$ seminal vesicle invasion
} 


\section{Staging Nomograms and Risk Groups}

The likelihood of OCPC according to the 2001 version of the Partin Tables was recorded on the basis of the serum PSA level, Gleason grade, and clinical staging (L.W. and K.K.) (10). Furthermore, the same coauthors divided the patients into three categories: low, intermediate, or high risk for cancer spread outside the prostate. The low-risk group had clinical stage T1 or T2 prostate cancer, with a Gleason score of 6 or less and a PSA level lower than $10 \mathrm{ng} / \mathrm{mL}$. The intermediate-risk group had clinical stage T1 or T2 prostate cancer, with a Gleason score of 7 and/or a PSA level of 10.1-20 ng/mL. The high-risk group had clinical stage T3 or T4 prostate cancer or a Gleason score greater than 7 or a PSA level greater than $20 \mathrm{ng} / \mathrm{mL}(20-22)$.

\section{Imaging and Interpretation}

Endorectal MR imaging and hydrogen 1 MR spectroscopic imaging had been performed by using a 1.5-T wholebody MR imager (Signa Horizon; GE Medical Systems, Milwaukee, Wis). Patients were examined in the supine position by using the body coil for excitation and a pelvic phased-array coil (GE Medical Systems) in combination with a commercially available balloon-covered expandable endorectal coil (Medrad, Pittsburgh, Pa) for signal reception. T1-weighted transverse and spin-echo MR images were obtained from the aortic bifurcation to the symphysis pubis by using the following parameters: repetition time msec/echo time msec, 700/8; section thickness, $5 \mathrm{~mm}$; intersection gap, 1 mm; field of view, 24 cm; matrix, 256 x 192; frequency direction, transverse (to prevent obstruction of the pelvic node by endorectal coil motion artifact); and one signal acquired. Thin-section, high-spatial-resolution transverse and coronal T2-weighted fast spin-echo MR images of the prostate and seminal vesicles were obtained by using the following parameters: repetition time msec/effective echo time msec, 5000/96; echo train length, 16; section thickness, $3 \mathrm{~mm}$; intersection gap, $0 \mathrm{~mm}$; field of view, $14 \mathrm{~cm}$; matrix, $256 \times 192$; frequency direction, anteroposterior (to prevent obstruction of the prostate by endorectal coil motion artifact); and three signals acquired. MR spectroscopic imaging was performed by using point-resolved spectroscopy voxel excitation, with band-selective inversion with gradient dephasing water and lipid suppression (23) and spatial encoding by means of chemical shift imaging (24) at 6.25-mm resolution in all three dimensions. Timing parameters were 1000/130, and imaging time was 17 minutes.

Data processing was performed at a workstation (Sun Ultra 10; Sun Microsystems, Mountain View, Calif) and included 2-Hz Lorentzian spectral apodization, four-dimensional Fourier transform, and automated frequency, phase, and baseline correction. Spectral data were zero filled to $3.1-\mathrm{mm}$ resolution in the superior-inferior dimension and overlaid on corresponding transverse T2-weighted MR images. Peak areas were calculated by using numeric integration. To provide a noise measurement, we calculated the standard deviation of the MR signal intensity in a region of the spectrum containing only noise. Metabolite peak areas were then normalized with respect to the noise standard deviation to yield an approximate signal-to-noise ratio.

MR studies had been interpreted prospectively by $12 \mathrm{MR}$ radiologists during their regular clinical assignment to the MR imaging service. All of the readers were trained in body MR imaging (nine had an MR imaging fellowship and the others had been involved with MR imaging since it was introduced to clinical practice). The level of experience reading MR images since fellowship ranged from 4 to 17 years among the readers. Each radiologist made his or her determination of MR prostate findings on the basis of his or her own continuous medical training and knowledge of previously described MR imaging features of extracapsular extension (ECE), seminal vesicle invasion (SVI), and lymph node metastasis (LNM) (16,25-31). All readers had access to the patients' medical records, including PSA level and biopsy findings as per their regular clinical practice. In addition, all readers had access to MR spectroscopic imaging data when available. The use of MR spectroscopic imaging data consisted of the evaluation of the location and number of abnormal voxels (voxels classified as suspicious or definitive for cancer by a spectroscopist). As described by Yu et al (25), when there are more than 4 voxels of cancer per section present at spectroscopy, the probability of ECE increases. Spectroscopy does not play a role in the assessment of the probability of SVI or LNM.

On the basis of the radiologists' written reports, the likelihood of ECE, SVI, and LNM was scored retrospectively by a single observer (L.W., 4 years of experience with research on MR imaging of the prostate). Three separate scores were assigned (one for ECE, one for SVI, and one for LNM) by using the following rating scale: score of 5, definite yes; score of 4, probable yes; score of 3, possible yes; score of 2, probable no; and score of 1, definite no. The greatest of the three scores was then used to calculate the likelihood of OCPC at MR evaluation with the following formula: OCPC $=6$ - maximal score (ie, the ECE, SVI, or LNM score). The rating scale for the likelihood of OCPC at MR evaluation was as follows: score of 1, definitely no OCPC; score of 2, probably no OCPC; score of 3, possible OCPC; score 
of 4, probable OCPC; and score of 5, definite OCPC. Thus, if the scores for ECE, SVI, and LNM were 1 (definitely no ECE), 1 (definitely no SVI), and 1 (definitely no LNM), respectively, the score for the likelihood of OCPC was 5 (definite OCPC). The formula is an attempt to assess one minus the probability of the patient's highest risk factor, though on a five-point ordinal scale. It was designed and validated by a statistician (M.W.K.) with extensive experience in prostate cancer outcome research.

\section{Histologic Evaluation}

Core biopsies had been evaluated for Gleason grade, greatest percentage of cancer in all biopsy cores, percentage of positive cores in all biopsy cores, and perineural invasion. Histology reports were reviewed by one author (L.W.). Radical prostatectomy specimens were examined by the institutional pathology department, as previously described by Yossepowitch et al (32). Specimens were fixed in formalin, with the external surface of the right and left sides dyed in two colors. The apical prostate was truncated perpendicular to the prostatic urethra and subsequently sectioned as slices parallel to the prostatic urethra. The bladder neck margin was obtained by sampling portions of soft tissue at the junction of the rough prostatic capsule and smooth bladder neck or most proximal portion of the submitted specimen corresponding to the anatomic bladder neck. The remaining prostate was completely transected at 3-5-mm intervals in a plane perpendicular to the urethra. The final pathology report following surgery was used to determine the presence of OCPC. OCPC was defined as the absence of cancer cells beyond the prostate capsule. Patients with LNM were considered not to have OCPC regardless of whether there was penetration through the prostate capsule.

\section{Statistical Analysis}

Logistic regression was used to estimate the relations between clinical variables and MR findings and the prediction of OCPC. Model discrimination was assessed by using the receiver operator characteristic (ROC) curves, which were plotted, calculated, and compared. When the staging nomograms were combined with MR results in the model, the jackknife method, a form of resampling that reduces the optimistic bias, was used to obtain the bias-corrected predicted probabilities and construct the ROC curves (33). The areas under the ROC curves were evaluated for models of OCPC prediction on the basis of the staging nomograms alone, MR findings alone (from endorectal MR imaging or combined endorectal MR imaging-MR spectroscopic imaging), and the staging nomograms plus MR findings (H.N.C., M.W.K.). To assess the incremental value of MR findings to the staging nomograms, the model for the prediction of OCPC based on the staging nomograms plus MR findings was compared with the model of

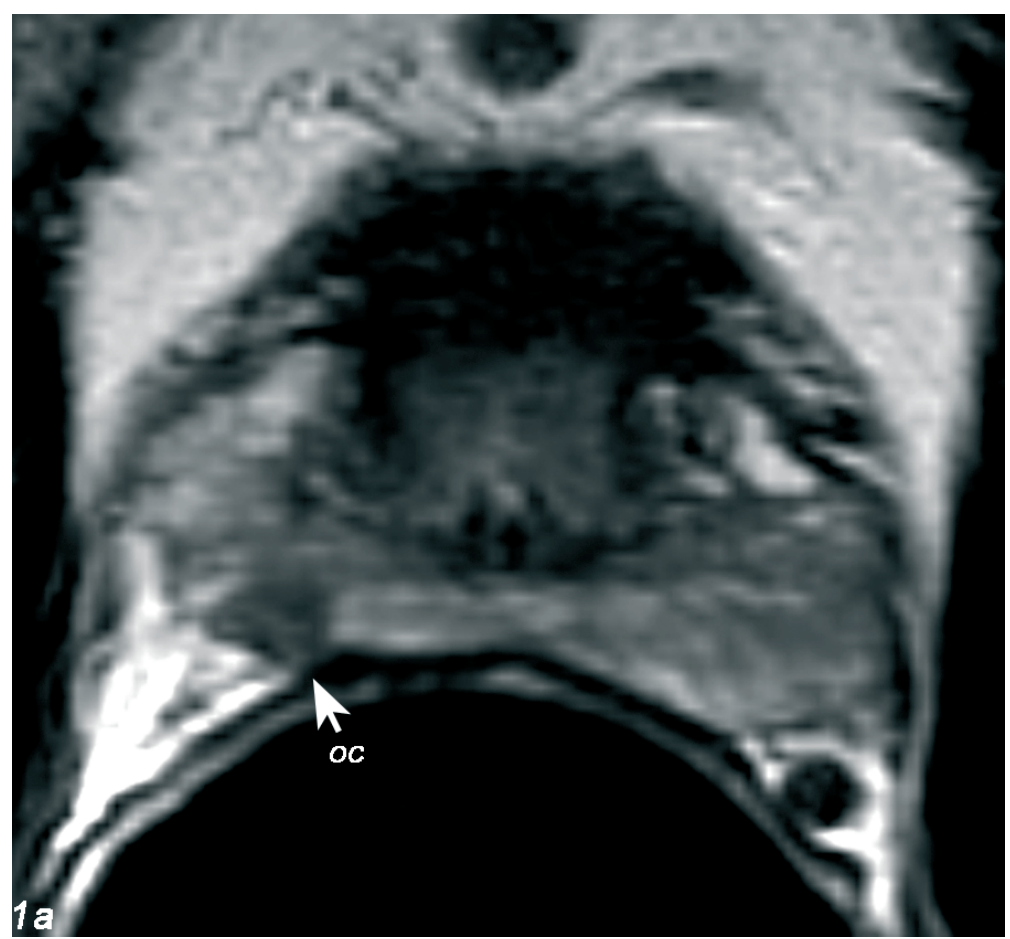

Figure 1a: Images in a 55-year-old man with a small palpable clinical stage T2 prostate nodule and PSA level of $0.80 \mathrm{ng} /$ $\mathrm{mL}$. Sextant biopsy results showed Gleason grade $3+3$ cancer involving 10\% of submitted tissue from the right side $(50 \%$ of the cores) and no perineural invasion. By using staging nomograms, the likelihood of OCPC was determined to be 81\%. (a-c) T2-weighted fast spin-echo MR images. (a) Transverse 3-mm-thick MR (4900/118) image shows OCPC (arrow) in the right apex. (b) Sagittal 4-mm-thick MR (6000/97) image shows a focus of cancer (arrow) in the right apex and midgland. (c) Coronal 3-mm-thick MR (4666/96) image shows cancer (arrow) in the right apex and midgland. (d) Whole-mount serial section of the removed prostate shows organ-confined cancer (arrow) involving the right posterior quadrant of the prostate. 

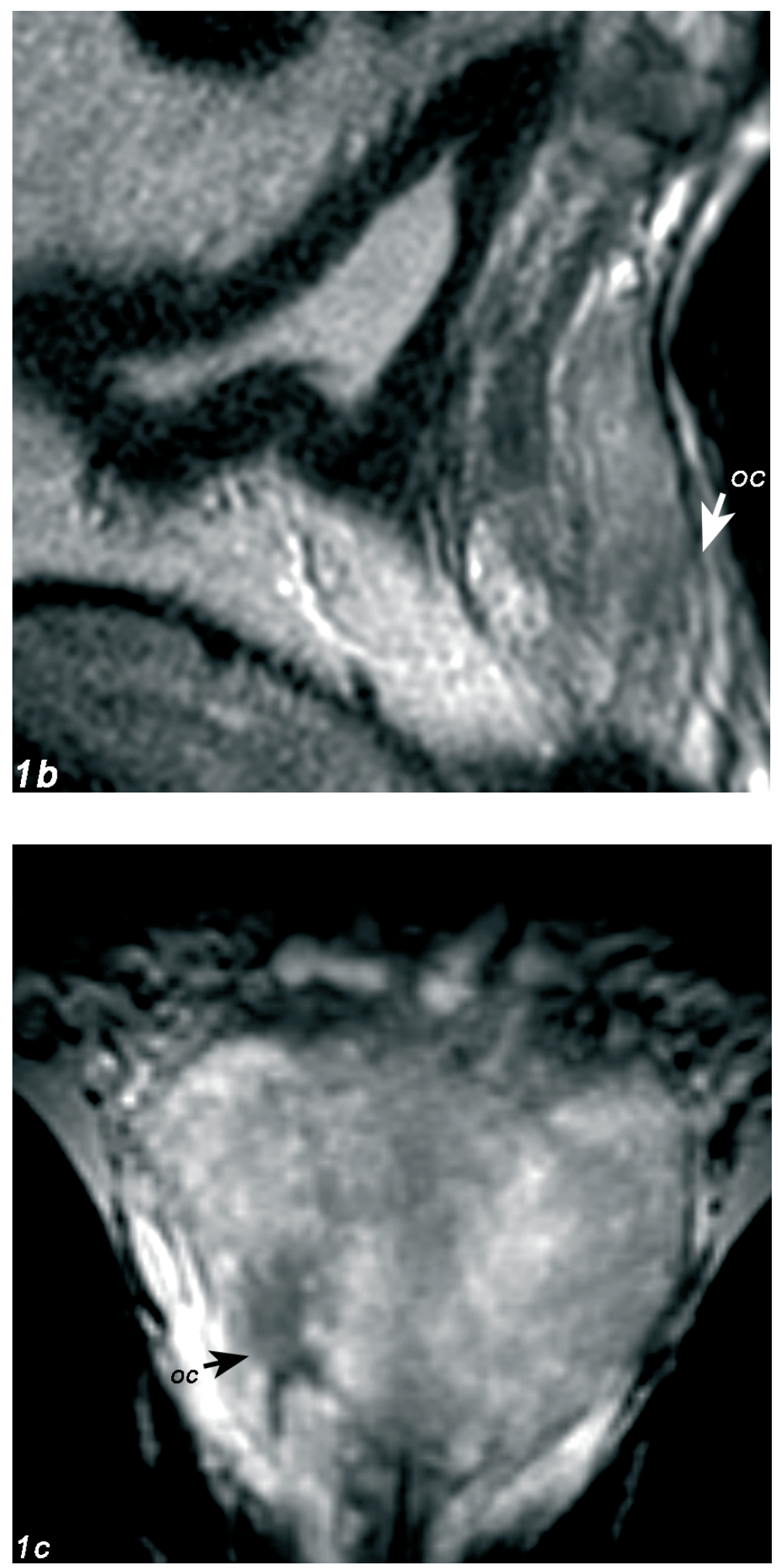

Figure 1b: Images in a 55-year-old man with a small palpable clinical stage $T 2$ prostate nodule and PSA level of $0.80 \mathrm{ng} / \mathrm{mL}$. Sextant biopsy results showed Gleason grade $3+3$ cancer involving $10 \%$ of submitted tissue from the right side $50 \%$ of the cores) and no perineural invasion. By using staging nomograms, the likelihood of OCPC was determined to be $81 \%$. (a-c) T2-weighted fast spin-echo MR images. (a) Transverse 3-mm-thick MR (4900/118) image shows OCPC (arrow) in the right apex. (b) Sagittal 4-mm-thick MR (6000/97) image shows a focus of cancer (arrow) in the right apex and midgland. (c) Coronal 3mm-thick MR (4666/96) image shows cancer (arrow) in the right apex and midgland. (d) Whole-mount serial section of the removed prostate shows organ-confined cancer (arrow) involving the right posterior quadrant of the prostate.
Figure 1c: Images in a 55-year-old man with a small palpable clinical stage T2 prostate nodule and PSA level of $0.80 \mathrm{ng} /$ $\mathrm{mL}$. Sextant biopsy results showed Gleason grade $3+3$ cancer involving $10 \%$ of submitted tissue from the right side $(50 \%$ of the cores) and no perineural invasion. By using staging nomograms, the likelihood of OCPC was determined to be $81 \%$. (a-c) T2-weighted fast spin-echo MR images. (a) Transverse 3-mm-thick MR (4900/118) image shows OCPC (arrow) in the right apex. (b) Sagittal 4-mm-thick MR (6000/97) image shows a focus of cancer (arrow) in the right apex and midgland. (c) Coronal 3mm-thick MR (4666/96) image shows cancer (arrow) in the right apex and midgland. (d) Whole-mount serial section of the removed prostate shows organ-confined cancer (arrow) involving the right posterior quadrant of the prostate. 


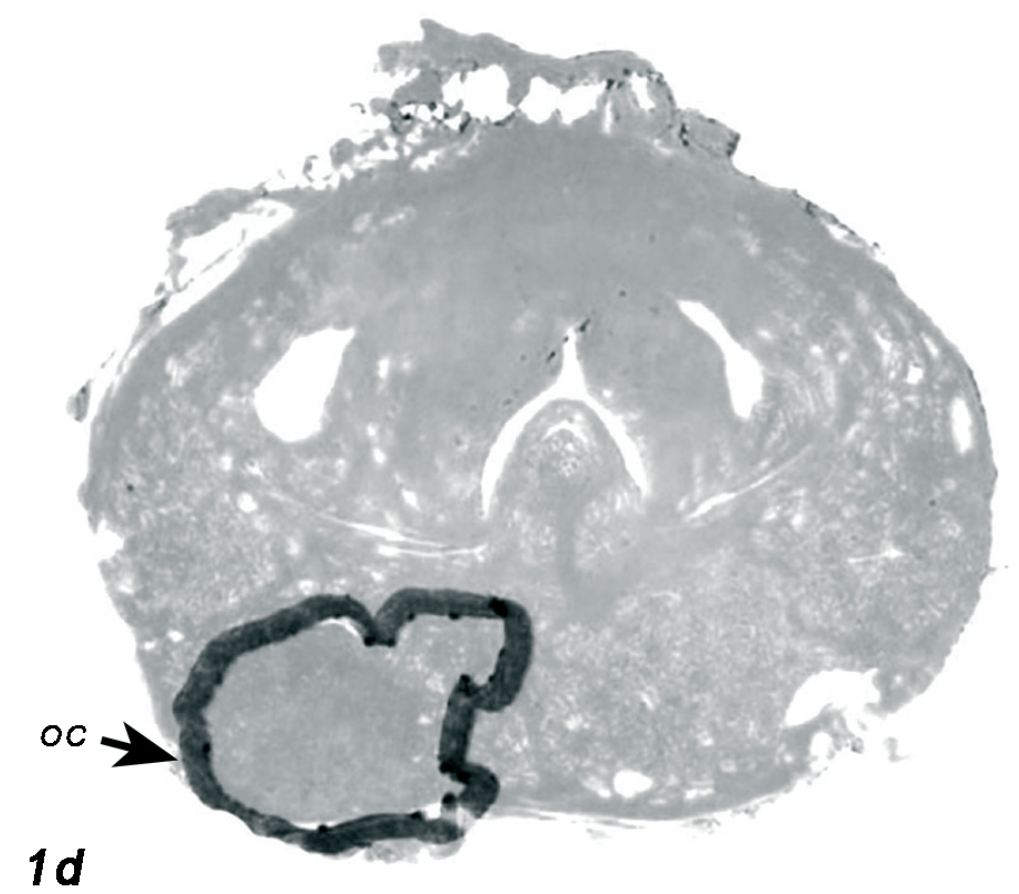

Figure 1d: Images in a 55-year-old man with a small palpable clinical stage T2 prostate nodule and PSA level of 0.80 $\mathrm{ng} / \mathrm{mL}$. Sextant biopsy results showed Gleason grade $3+3$ cancer involving $10 \%$ of submitted tissue from the right side ( $50 \%$ of the cores) and no perineural invasion. By using staging nomograms, the likelihood of OCPC was determined to be $81 \%$. (a-c) T2-weighted fast spinecho MR images. (a) Transverse 3-mmthick MR (4900/118) image shows OCPC (arrow) in the right apex. (b) Sagittal 4mm-thick MR (6000/97) image shows a focus of cancer (arrow) in the right apex and midgland. (c) Coronal 3-mm-thick MR (4666/96) image shows cancer (arrow) in the right apex and midgland. (d) Whole-mount serial section of the removed prostate shows organ-confined cancer (arrow) involving the right posterior quadrant of the prostate.

(Color version available in chapter 18).

\section{Table 1. Distribution of Preoperative Clinical Variables}

\begin{tabular}{|lc}
\hline Clinical Variable & Finding \\
\hline PSA level $^{*+}$ & $\begin{array}{c}5.64 \pm 7.82 \\
(0.10-13.40)\end{array}$ \\
\hline Gleason grade & $377(62)$ \\
\hline $3+3$ & $145(24)$ \\
\hline $3+4$ & $48(8)$ \\
\hline $4+3$ & $10(2)$ \\
\hline $4+4 / 3+5$ & $10(2)$ \\
\hline $4+5$ & $2(0)$ \\
\hline $5+5$ & $0.21 \pm 0.27$ \\
\hline Greatest percentage of cancer ${ }^{*}$ & $(0.01-1.00)$ \\
\hline Percentage of positive cores ${ }^{* 5}$ & $0.38 \pm 0.26$ \\
\hline Perineural invasion & $(0.04-1.00)$ \\
\hline Absent & \\
\hline Present & $530(87)$ \\
\hline Clinical stage & $82(13)$ \\
\hline T1 & $0(0)$ \\
\hline T2 & $371(61)$ \\
\hline T3a & $241(39)$ \\
\hline
\end{tabular}

Note.-Unless otherwise indicated, data are numbers of patients, and data in parentheses are percentages.

* Data are median results \pm standard deviations, and data in parentheses are ranges.

† Results obtained in all 612 patients.

₹ Greatest percentage of cancer in all biopsy cores (results obtained in 599 patients).

$\S$ Percentage of positive cores in all biopsy cores (results obtained in 539 patients).

|| Indicates lymph node metastasis. 
Table 2. Predicted Probability of OCPC according to the 2001 Partin Tables

\begin{tabular}{lcccc} 
& & \multicolumn{3}{c}{ Finding } \\
\cline { 3 - 5 } Risk Group & No. of Patients & Mean & Standard Deviation & Range \\
\hline Low risk & 332 & 0.77 & 0.41 & $0.46-0.9$ \\
Intermediate risk & 224 & 0.45 & 0.18 & $0.07-0.8$ \\
High risk & 56 & 0.29 & 0.16 & $0.06-0.62$
\end{tabular}

Table 3. Final Pathologic Stage

\begin{tabular}{lc} 
TNM Stage & No. of Patients \\
\hline pT1 & $2(0)$ \\
pT2 & $443(72)$ \\
pT3a & $115(19)$ \\
pT3b & $38(6)$ \\
pT4 & $14(2)$ \\
pTanyN1* & $30(5)$ \\
\hline
\end{tabular}

Note.-Data in parentheses are percentages. Staging is according to the 2002 American Joint Committee on Cancer.

* Indicates lymph node metastasis.

Table 4. Areas under ROC Curves of OCPC Prediction Models

\begin{tabular}{|c|c|c|c|c|c|}
\hline Patient Group & $\begin{array}{c}\text { No. of } \\
\text { Patients }\end{array}$ & $\begin{array}{c}\text { MR } \\
\text { Findings }\end{array}$ & $\begin{array}{l}\text { Partin } \\
\text { Tables }\end{array}$ & $\begin{array}{l}\text { MR Findings } \\
\text { and Partin Tables* }\end{array}$ & $P_{\text {Value }}^{\dagger}$ \\
\hline All & 612 & 0.81 & 1.80 & 0.88 & $<.01$ \\
\hline \multicolumn{6}{|l|}{ MR group ${ }^{\ddagger}$} \\
\hline Endorectal imaging only & 229 & 0.77 & 0.79 & 0.84 & .045 \\
\hline Combined imaging & 383 & 0.84 & 0.81 & 0.90 & $<.01$ \\
\hline \multicolumn{6}{|l|}{ Risk Group } \\
\hline Low & 332 & 0.76 & 0.60 & 0.78 & .02 \\
\hline Intermediate & 224 & 0.80 & 0.70 & 0.83 & $<.01$ \\
\hline High & 56 & 0.83 & 0.74 & 0.87 & $<.01$ \\
\hline
\end{tabular}

* Jackknife method used.

$+P$ values are for Partin Tables alone versus MR findings and Partin Tables.

₹ Patients underwent endorectal coil MR imaging alone or in combination with MR spectroscopic imaging. 
Index

Partin OCPC + MR OCPC $\{$ AUC $=0.876\}$

Partin OCPC $\{A U C=0.800\}$

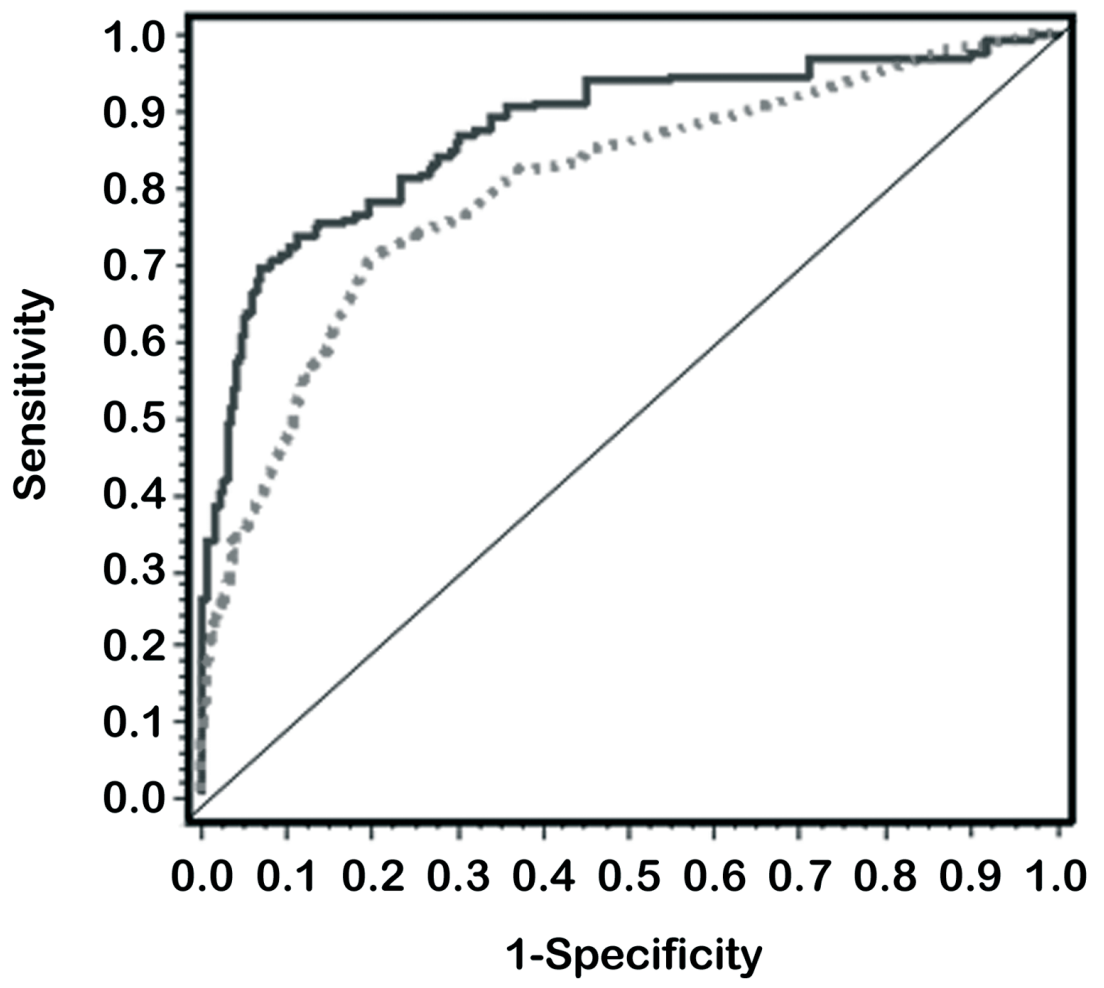

Figure 2: Graph shows ROC curvesforjackknife-predicted probabilities of OCPC for two models for the study population: one based only on clinical staging nomograms (Partin OCPC) and one based on staging nomograms plus MR findings (Partin OCPC + MR OCPC). The model with MR findings has a significantly greater area under the ROC curve $(A \cup C)$ than does the model lacking MR findings (0.876 vs $0.80, P<.01$ ).

prediction based on the staging nomograms alone. $\mathrm{P}<.05$ was considered to indicate a statistically significant difference. Software programs used for data analysis were SAS (version 8.2; SAS Institute, Cary, NC) and S-PLUS (version 2000; Insightful, Seattle, Wash).

\section{RESULTS}

At surgical histopathologic evaluation, 445 (73\%) of 612 patients had evidence of OCPC (Fig 1). Table 1 summarizes the distribution of the preoperative clinical variables, Table 2 shows the predicted probabilities of OCPC (derived from the 2001 Partin Tables) according to risk group, and Table 3 demonstrates the distribution of final pathologic staging.

Overall, in the prediction of OCPC, the area under the ROC curve for the staging nomograms was 0.80 , while the area under the ROC curve for the staging nomograms plus MR findings was 0.88; the difference was significant (P $<$.01) (Table 4) (Fig 2).

\section{Risk Groups}

The incremental value of MR findings to the staging nomograms was significant in all three risk groups, although it was greater in the intermediate- and high-risk groups $(P<.01$ for both) than in the low-risk group $(P=$ .02) (Table 4). 
Table 5. Accuracy of MR Imaging Prediction of Prostate Cancer with and without MR Spectroscopic Imaging

\begin{tabular}{|c|c|c|c|}
\hline Risk Group and Type of MR* & $\begin{array}{l}\text { No. of } \\
\text { Patients }\end{array}$ & $\begin{array}{l}\text { Area under the } \\
\text { ROC Curve }^{+}\end{array}$ & $P$ Value \\
\hline \multicolumn{4}{|l|}{ All } \\
\hline Combined & 383 & $0.84(0.79,0.89)$ & $>.05$ \\
\hline Endorectal imaging only & 229 & $0.77(0.70,0.84)$ & \\
\hline \multicolumn{4}{|l|}{ Low } \\
\hline Combined & 206 & $0.81(0.69,0.92)$ & $>.05$ \\
\hline Endorectal imaging only & 126 & $0.72(0.59,0.85)$ & \\
\hline \multicolumn{4}{|l|}{ Intermediate } \\
\hline Combined & 146 & $0.84(0.77,0.90)$ & $>.05$ \\
\hline Endorectal imaging only & 78 & $0.74(0.63,0.85)$ & \\
\hline \multicolumn{4}{|l|}{ High } \\
\hline Combined & 31 & $0.85(0.71,0.99)$ & $>.05$ \\
\hline Endorectal imaging only & 25 & $0.80(0.66,0.95)$ & \\
\hline
\end{tabular}

* Patients underwent endorectal coil MR imaging alone or in combination with MR spectroscopic imaging. † Data in parentheses are $95 \%$ confidence intervals.

\section{MR Spectroscopic Imaging and Endorectal MR Imaging}

In the combined endorectal MR imaging-MR spectroscopic imaging group, the areas under the ROC curves were 0.81 for the staging nomograms and 0.90 for the staging nomograms plus MR findings; the difference was significant $(\mathrm{P}<.01)$ (Table 4).

Prediction of OCPC with MR findings plus the staging nomograms was better in the endorectal MR imaging-MR spectroscopic imaging group (area under the ROC curve, 0.84 ; 95\% confidence interval: $0.79,0.89$ ) than in the endorectal MR imaging-only group (area under the ROC curve, $0.77 ; 95 \%$ confidence interval: $0.70,0.84$ ), but the difference was not significant $(P>.05)$ (Table 4). The incremental value of MR spectroscopic imaging to endorectal MR imaging was slightly greater in the low- and intermediate-risk groups than in the high-risk group (Table 5), but the difference was not significant in any of the risk groups.

\section{Discussion}

Pretreatment knowledge of OCPC is important for treatment selection and planning, regardless of whether the treatment method ultimately chosen is watchful waiting, surgery, or radiation therapy. Radical prostatectomy in patients with pathologically organ-confined cancer results in a survival rate comparable with that of age-matched controls without prostate cancer (34-39). Since the introduction of the Partin Tables in 1997, investigators have repeatedly validated the nomograms' capacity to help predict the pathologic stage of clinically localized prostate cancer (11-13). In 2001, the nomograms were updated based on a more contemporary cohort of disease features (10). The accuracy of the staging nomograms in predicting OCPC is high, with reports of the area under the ROC curve ranging from 0.79 to $0.82(12,13)$. Our study results demonstrated similar accuracy for the staging nomograms in predicting OCPC, with an overall area under the ROC curve of 0.80 . Despite the strong predictive ability and the cost-effectiveness of the staging nomograms, there is room for improved accuracy of prediction, particularly since clinical staging in the staging nomograms is based only on digital rectal examination. Moreover, the staging nomograms cannot assist in the localization of ECE, which is critical for optimal treatment planning $(5,9)$.

Debate persists regarding whether MR imaging should be used routinely for presurgical evaluation of prostate cancer. Because of the high incidence of prostate cancer and the high cost of MR examinations, it has been stated that routine use of endorectal MR imaging alone or in combination with MR spectroscopic imaging might merely 
add a financial burden to the health care system unless it prevents unnecessary surgery or improves treatment planning and outcomes (40). It has been shown that endorectal MR imaging adds value in all risk groups $(5,41)$; in the prediction of ECE, the greatest incremental value of endorectal MR imaging to the Partin Tables has been found in high-risk patients $(14,16)$. In our study, the addition of MR findings to the Partin Tables (2001 version) significantly improved the prediction of OCPC for the overall patient population $(P<.01)$. The addition of MR findings also increased the area under the ROC curve significantly in the low-risk group $(P=.02)$ and in the intermediate- and high-risk groups $(\mathrm{P}<.01$ for both).

The contribution of MR spectroscopic imaging findings to endorectal MR imaging in the prediction of OCPC was also assessed in our study. The magnitude and extent of metabolic abnormality on MR spectroscopic images is indicative of tumor aggressiveness, volume, and stage $(42,43)$. Yu et al $(25)$ demonstrated that the combined use of endorectal MR imaging and MR spectroscopic imaging decreased interobserver variability and, for less experienced radiologists, significantly improved the detection of ECE. In our study, the accuracy of radiologists' predictions of OCPC was higher in the combined MR group than in the endorectal MR imaging-only group, but the difference was not significant; MR findings contributed significant incremental value to the staging nomograms in the prediction of OCPC in both of these groups.

Our study was limited by the fact that it was designed to assess the value of MR readings as used in clinical practice; the readers were not blinded to clinical data such as PSA level and biopsy results. Furthermore, only one reader evaluated each case once, so we could not assess interobserver and intraobserver variability. With respect to the assessment of the incremental value of MR spectroscopic imaging, separate readings with and without MR spectroscopic imaging were not performed and therefore the true incremental value of MR spectroscopic imaging needs further analysis.

In conclusion, although further multicenter confirmatory studies would be helpful, our results show that MR findings (from endorectal MR imaging or combined endorectal MR imaging-MR spectroscopic imaging) contribute significant incremental value to clinical staging nomograms in the prediction of OCPC. The incorporation of endorectal MR imaging into future staging nomograms for the prediction of OCPC may therefore be warranted.

\section{References}

1. Jemal A, Murray T, Ward E, et al. Cancer statistics, 2005. CA Cancer J Clin 2005;55:10-30.

2. Ung JO, Richie JP, Chen MH, Renshaw AA, D'Amico AV. Evolution of the presentation and pathologic and biochemical outcomes after radical prostatectomy for patients with clinically localized prostate cancer diagnosed during the PSA era. Urology 2002;60:458-463.

3. Catalona WJ, Ramos CG, Carvalhal GF. Contemporary results of anatomic radical prostatectomy. CA Cancer J Clin 1999;49:282296.

4. Gohji K, Okamoto M, Morisue K, Fujii A. Usefulness of digital rectal examination, serum prostate-specific antigen, transrectal ultrasonography and systematic prostate biopsy for the detection of organ-confined prostate cancer. Int J Urol 1995;2:116120.

5. Poulakis $V$, Witzsch $U$, De Vries $R$, et al. Preoperative neural network using combined magnetic resonance imaging variables, prostate specific antigen and Gleason score to predict prostate cancer stage. J Urol 2004;172:1306-1310.

6. Ogawa O, Egawa S, Arai Y, Tobisu K, Yoshida O, Kato T. Preoperative predictors for organ-confined disease in Japanese patients with stage T1c prostate cancer. Int J Urol 1998;5:454-458.

7. Ennis RD, Flynn SD, Fischer DB, Peschel RE. Preoperative serum prostate-specific antigen and Gleason grade as predictors of pathologic stage in clinically organ confined prostate cancer: implications for the choice of primary treatment. Int J Radiat Oncol Biol Phys 1994;30:317-322.

8. Catalona WJ, Smith DS, Ratliff TL, Basler JW. Detection of organ-confined prostate cancer is increased through prostate-specific antigenbased screening. JAMA 1993;270:948-954.

9. Partin AW, Kattan MW, Subong EN, et al. Combination of prostate-specific antigen, clinical stage, and Gleason score to predict pathological stage of localized prostate cancer: a multi-institutional update. JAMA 1997;277:1445-1451.

10. Partin AW, Mangold LA, Lamm DM, Walsh PC, Epstein JI, Pearson JD. Contemporary update of prostate cancer staging nomograms (Partin Tables) for the new millennium. Urology 2001;58:843-848.

11. Blute ML, Bergstralh EJ, Partin AW, et al. Validation of Partin tables for predicting pathological stage of clinically localized prostate cancer. J Urol 2000;164:1591-1595.

12. Augustin H, Eggert T, Wenske S, et al. Comparison of accuracy between the Partin Tables of 1997 and 2001 to predict final pathological stage in clinically localized prostate cancer. J Urol 2004;171:177-181.

13. Graefen M, Augustin H, Karakiewicz PI, et al. Can predictive models for prostate cancer patients derived in the United States of America be utilized in European patients? a validation study of the Partin tables. Eur Urol 2003;43:6-11.

14. Hricak $H$, Wang $L$, Wei DC, et al. The role of preoperative endorectal magnetic resonance imaging in the decision regarding whether to preserve or resect neurovascular bundles during radical retropubic prostatectomy. Cancer 2004;100:2655-2663. 
15. Hricak $H$, Schoder $H$, Pucar D, et al. Advances in imaging in the postoperative patient with a rising prostate-specific antigen level. Semin Oncol 2003;30:616-634.

16. Wang L, Mullerad M, Chen HN, et al. Prostate cancer: incremental value of endorectal MR imaging findings for prediction of extracapsular extension. Radiology 2004;232:133-139.

17. Mullerad M, Hricak H, Wang L, Chen HN, Kattan MW, Scardino PT. Prostate cancer: detection of extracapsular extension by genitourinary and general body radiologists at MR imaging. Radiology 2004;232:140-146.

18. Qayyum A, Coakley FV, Lu Y, et al. Organ-confined prostate cancer: effect of prior transrectal biopsy on endorectal MRI and MR spectroscopic imaging. AJR Am J Roentgenol 2004;183:1079-1083.

19. Claus FG, Hricak H, Hattery RR. Pretreatment evaluation of prostate cancer: role of MR imaging and $1 \mathrm{H}$ MR spectroscopy. RadioGraphics 2004;24(suppl 1):S167-S180.

20. D'Amico AV, Schnall M, Whittington R, et al. Endorectal coil magnetic resonance imaging identifies locally advanced prostate cancer in select patients with clinically localized disease. Urology 1998;51:449-454.

21. Hermansen DK, Whitmore WF Jr. Frozen section lymph node analysis in pelvic lymphadenectomy for prostate cancer. J Urol 1988;139:10731074.

22. Khan MA, Partin AW. Management of high-risk populations with locally advanced prostate cancer. Oncologist 2003;8:259-269.

23. Star-Lack J, Nelson SJ, Kurhanewicz J, Huang LR, Vigneron DB. Improved water and lipid suppression for 3D PRESS CSI using RF band selective inversion with gradient dephasing (BASING). Magn Reson Med 1997;38:311-321.

24. Brown TR, Kincaid BM, Ugurbil K. NMR chemical shift imaging in three dimensions. Proc Natl Acad Sci U S A 1982;79:3523-3526.

25. Yu KK, Scheidler J, Hricak H, et al. Prostate cancer: prediction of extracapsular extension with endorectal MR imaging and threedimensional proton MR spectroscopic imaging. Radiology 1999;213:481-488.

26. Sala E, Akin O, Moskowitz CS, et al. The role of endorectal MR imaging in the evaluation of seminal vesicle invasion: diagnostic accuracy and multivariate feature analysis. Radiology (in press).

27. Wang L, Hricak H, Kattan MW, et al. Combined endorectal and phased array MRI in the prediction of pelvic lymph node metastasis in prostate cancer. AJR Am J Roentgenol (in press).

28. David V. MR imaging of the prostate and seminal vesicles. Magn Reson Imaging Clin N Am 1996;4:497-518.

29. Secaf E, Nuruddin RN, Hricak H, McClure RD, Demas B. MR imaging of the seminal vesicles. AJR Am J Roentgenol 1991;156:989994.

30. Harisinghani MG, Barentsz J, Hahn PF, et al. Noninvasive detection of clinically occult lymph-node metastases in prostate cancer. N Engl J Med 2003;348:2491-2499.

31. Tempany CM, Zhou X, Zerhouni EA, et al. Staging of prostate cancer: results of Radiology Diagnostic Oncology Group project comparison of three MR imaging techniques. Radiology 1994;192:47-54.

32. Yossepowitch $\mathrm{O}$, Sircar K, Scardino PT, et al. Bladder neck involvement in pathological stage pT4 radical prostatectomy specimens is not an independent prognostic factor. J Urol 2002;168:2011-2015.

33. Efron B, Tibshirani RJ. An introduction to the bootstrap. New York, NY: Chapman \& Hall, 1993.

34. Graefen M, Haese A, Pichlmeier U, et al. A validated strategy for side specific prediction of organ confined prostate cancer: a tool to select for nerve sparing radical prostatectomy. J Urol 2001;165:857-863.

35. Do V, Choo R, De Boer G, et al. The role of serial free/total prostate-specific antigen ratios in a watchful observation protocol for men with localized prostate cancer. BJU Int 2002;89:703-709.

36. Hoff B, Pow-Sang JM. Observation in the management of localized prostate cancer. Cancer Control 2001;8:151-154.

37. Brasso K, Friis S, Juel K, Jorgensen T, Iversen P. Mortality of patients with clinically localized prostate cancer treated with observation for 10 years or longer: a population based registry study. J Urol 1999;161:524-528.

38. Moon TD, Brawer MK, Wilt TJ. Prostate Intervention Versus Observation Trial (PIVOT): a randomized trial comparing radical prostatectomy with palliative expectant management for treatment of clinically localized prostate cancer. PIVOT Planning Committee. J Natl Cancer Inst Monogr 1995;19:69-71.

39. D'Amico AV, Whittington R, Malkowicz SB, et al. A multivariate analysis of clinical and pathological factors that predict for prostate specific antigen failure after radical prostatectomy for prostate cancer. J Urol 1995;154:131-138.

40. Penson DF, Litwin MS, Aaronson NK. Health related quality of life in men with prostate cancer. J Urol 2003;169:1653-1661.

41. Bostwick DG, Qian J, Schlesinger C. Contemporary pathology of prostate cancer. Urol Clin North Am 2003;30:181-207.

42. Kurhanewicz J, Vigneron DB, Nelson SJ. Three-dimensional magnetic resonance spectroscopic imaging of brain and prostate cancer. Neoplasia 2000;2:166-189.

43. Zakian KL, Sircar K, Hricak H, et al. Correlation of proton MR spectroscopic imaging with Gleason score based on step-section pathologic analysis after radical prostatectomy. Radiology 2005;234:804-814. 
2 
Prostate Cancer: Incremental Value of Endorectal MR Imaging Findings for Prediction of Extracapsular Extension 


\section{Prostate Cancer: Incremental Value of Endorectal MR Imaging Findings for Prediction of Extracapsular Extension}

\section{Supported by National Institutes of Health grant R01 CA76423.}

Liang Wang, Michael Mullerad, Hui-Ni Chen, Steven C. Eberhardt, Michael W. Kattan, Peter T. Scardino and Hedvig Hricak

From the Departments of Radiology (L.W., H.N.C., S.C.E., H.H.) and Urology (M.M., M.W.K., P.T.S.), Memorial Sloan-Kettering Cancer Center, 1275 York Ave, New York, NY 10021. From the 2002 RSNA scientific assembly. Received July 10, 2003; revision requested September 16; revision received December 19; accepted January 13, 2004.

\section{Abstract}

Purpose: To assess the incremental value of endorectal magnetic resonance (MR) imaging findings in addition to clinical variables for prediction of extracapsular extension (ECE) in patients with prostate cancer.

Materials and Methods: In this cohort study, 344 consecutive patients with biopsy-proved prostate cancer underwent endorectal MR imaging prior to surgery; 216 of these patients also underwent MR spectroscopic imaging. MR images were interpreted by 10 attending radiologists. The likelihood of ECE was scored retrospectively on the basis of MR imaging reports. Clinical variables included serum prostate-specific antigen (PSA) level, Gleason score, clinical stage of tumor, greatest percentage of cancer in all core biopsy specimens, percentage of cancer-positive core specimens in all core biopsy specimens, and presence of perineural invasion. For data analysis, receiver operating characteristic (ROC) curves and univariate and multivariate logistic regression analyses were used. Jackknife analysis was used for prediction of probability from a model that included clinical variables as tested comparatively with a model that included the clinical variables plus endorectal MR imaging findings. A difference with $\mathrm{P}<.05$ was considered significant.

Results: At univariate analysis, all variables were associated with ECE. At ROC univariate analysis, endorectal MR imaging findings had the largest area under the ROC curve. At multivariate analysis, serum PSA level, percentage of cancer in all core biopsy specimens, and endorectal MR imaging findings $(P=.001, P=.001$, and $P<.001$, respectively) were predictors of ECE. Areas under ROC curve for two models, with and without endorectal MR imaging findings, were 0.838 and 0.772 , respectively $(P=.022)$.

Conclusion: A model containing endorectal MR imaging findings has a significantly larger area under the ROC curve than a model containing only clinical variables; thus, endorectal MR imaging findings add incremental value in the prediction of ECE.

(c) RSNA, 2004 


\section{INTRODUCTION}

$\mathbf{P}$

rostate cancer continues to be the second leading cause of cancer death in American men. In 2003, 28,900 men were expected to die from the disease (1). In the past decade, because of screening with prostate-specific antigen (PSA) level, there has been a dramatic downward trend in the stage of prostate cancer determined at the time of diagnosis (2). As demonstrated by the Surveillance, Epidemiology, and End Results Program of the National Cancer Institute, in 2003, 85\% of prostate cancers were local or regional at diagnosis, compared with $72 \%$ in 1993 (1,3). This change in stage at diagnosis brings new challenges to the presurgical detection of extracapsular extension (ECE) in patients who are at risk for it.

Various methods for the prediction of ECE have been proposed. The established presurgical clinical variables for the prediction of pathologic stage are clinical tumor stage, which includes digital rectal examination findings; serum PSA level; and Gleason score (4-6). Nomograms that are based on presurgical data were introduced to help evaluate patient risk for ECE, seminal vesicle invasion, and metastasis to lymph nodes, as well as 5-year freedom from cancer recurrence. These models have high accuracy for prediction with either aggressive or clinically indolent tumors. The assessment of additional presurgical variables to enhance the specificity and sensitivity of the present models is of great interest.

The aim of this study was to assess the incremental value of endorectal magnetic resonance (MR) imaging findings in addition to clinical variables for prediction of ECE in patients with prostate cancer.

\section{Materials and Methods}

\section{Patients}

Between May 1999 and January 2003, 344 consecutive patients with prostate cancer were referred from the urology department at our institution to undergo endorectal MR imaging before they underwent radical retropubic prostatectomy. Two hundred sixteen patients underwent endorectal MR imaging combined with MR spectroscopic imaging, and 128 patients underwent endorectal MR imaging alone. Approval of the institutional review board was obtained, and informed consent was obtained from all patients.

Mean patient age was 57.5 years (range, 32-74 years). None of the patients received neoadjuvant hormonal or radiation therapy prior to surgery. Tissue diagnosis of prostate cancer was established with biopsy specimens in all patients. Clinical and MR imaging data were recorded retrospectively from the patients' medical records by one author (L.W.). Data in the same patient cohort are reported in the study by Mullerad et al (7).

\section{Imaging and Image Interpretation}

Endorectal MR imaging and hydrogen 1 MR spectroscopic imaging were performed with a 1.5-T whole-body MR imaging unit (Signa Horizon; GE Medical Systems, Milwaukee, Wis). The examination was performed with patients in the supine position. A body coil was used for excitation, and a pelvic phased-array coil (GE Medical Systems) combined with a commercially available balloon-covered expandable endorectal coil (Medrad, Pittsburgh, Pa) was used for signal reception. Transverse T1-weighted and spin-echo MR images were obtained from the aortic bifurcation to the symphysis pubis with the following parameters: repetition time msec/echo time msec, 700/8; section thickness, 5 mm; intersection gap, 1 mm; field of view, 24 cm; matrix, $256 \times 192$; and frequency direction, transverse (to prevent obstruction of the pelvic node from endorectal coil motion artifact). One signal was acquired.

Transverse and coronal thin-section high-spatial-resolution T2-weighted fast spin-echo MR images of the prostate and seminal vesicle were obtained with the following parameters: 5,000/96 (effective); echo train length, 16; section thickness, 3 mm; intersection gap, 0 mm; field of view, 14 cm; matrix, 256 × 192; frequency direction, anteroposterior (to prevent obstruction of the prostate from endorectal coil motion artifact); and number of signals acquired, three.

\section{${ }^{1}$ Abbreviations}

$\mathrm{ECE}=$ extracapsular extension, $\mathrm{PNI}=$ perineural invasion, $\mathrm{PSA}=$ prostate-specific antigen, $\mathrm{ROC}=$ receiver operating characteristic 
MR spectroscopic imaging was performed by using point-resolved spectroscopic voxel excitation (8), band-selective inversion with gradient dephasing water and lipid suppression (9), and spatial encoding with chemical shift imaging (10) at 6.25-mm resolution in all three dimensions (left-right, anterior-posterior, superior-inferior dimensions). Parameters were 1,000/130, and imaging time was 17 minutes.

Data processing was performed at a workstation (Sun Ultra 10; Sun Microsystems, Mountain View, Calif) and included 2-Hz lorentzian spectral apodization; four-dimensional Fourier transform; and automated frequency, phase, and baseline correction (11). Spectral data were zero filled to 3.1-mm resolution in the superior-inferior dimension and overlaid on corresponding transverse T2-weighted MR images. Peak areas were calculated by using numeric integration. To provide a noise measurement, we calculated the SD of the MR signal intensity in a region of the spectrum containing only noise. Metabolite peak areas were then normalized with respect to the noise SD to yield an approximate signal-to-noise ratio.

MR images were interpreted by 10 body MR imaging radiologists (including H.H., S.C.E.) during their clinical assignment to the MR imaging service. The readers' experience in interpretation of clinical MR images since fellowship ranged from 4 to more than 15 years. Official pretreatment MR image readings were used for the data analysis. There was no initial meeting or training to establish the criteria for ECE. Rather, radiologists made their determinations on the basis of their own continuing medical training and knowledge of previously described MR imaging features of ECE. The diagnostic criteria used by the radiologists to determine ECE on endorectal MR images included irregular capsular bulge, periprostatic fat infiltration, obliteration of the rectoprostatic angle, and asymmetry or direct involvement of the neurovascular bundles (12). MR spectroscopic imaging results, when available, were provided for all readers. The extent to which MR spectroscopic imaging data were used in image interpretation differed among radiologists. On the basis of the radiologists' written reports, one author (L.W.) retrospectively scored the likelihood for ECE with a five-point scale as follows: score 1, no ECE; score 2, probably no ECE (cannot be ruled out though there is no clear evidence of it); score 3, possible ECE (a lesion is suspected of demonstrating ECE); score 4, probable ECE (a lesion is highly suspected of demonstrating ECE); score 5, definite ECE.

\section{Histologic Analysis}

Histologic analysis reports at core biopsy were evaluated for Gleason score, greatest percentage of cancer in all core biopsy specimens, percentage of cancer-positive core specimens in all core biopsy specimens (the number of cancer-positive core specimens divided by the total number of core biopsy specimens), and presence of perineural invasion (PNI). The greatest percentage of cancer was determined in each patient by examining each core biopsy specimen and dividing the length of the core specimen tissue with cancer by the whole core specimen length; the core specimen with the highest percentage of cancer defined the patient's greatest percentage of cancer. This parameter has been previously described by Rubin et al (13) and Bismar et al (14).

Specimens removed at radical prostatectomy were examined in the pathology department at our institution, as previously described by Yossepowitch et al (15). In short, specimens were fixed in formalin, with the external surface of the right and left sides inked with two colors. The apical prostate was truncated perpendicular to the prostatic urethra and was subsequently sectioned as slices parallel to the prostatic urethra. The bladder neck margin was obtained by sampling portions of soft tissue at the junction of the rough prostatic capsule and smooth bladder neck or the most proximal portion of the submitted specimen that corresponded to the anatomic bladder neck. The remaining prostate was completely transected at 3-5-mm intervals in a plane perpendicular to the urethra. The final pathologic report following surgery was used to determine the presence of ECE. The presence of cancer cells beyond the capsular margin was used as the definition of ECE.

\section{Statistical Analysis}

Both univariate and multivariate analyses were performed for all clinical and imaging variables tested. Predictor variables that we tested included serum PSA level, Gleason score, clinical stage of tumor, greatest percentage of cancer in all core biopsy specimens, percentage of cancer-positive core specimens in all core biopsy specimens, and presence of PNI. With receiver operating characteristic (ROC) univariate analysis, the incremental value of MR spectroscopic imaging findings was assessed by comparing findings in the group of 216 patients who underwent both endorectal MR imaging and MR spectroscopic imaging with those in the group of 128 patients who underwent only endorectal MR imaging. 
We evaluated the area under the ROC curve for each variable. In addition, we compared two models: one that contained all clinical variables plus endorectal MR imaging findings and another that contained only the clinical variables. To judge the value of endorectal MR imaging findings as a marker, the predictions analyzed with jackknife methods from these models were compared for their ability to predict ECE. By using bootstrapping for bias correction, a P value was derived to test for a difference in the predictive ability of these two models (16). A difference with $\mathrm{P}<.05$ was considered significant. Software programs (SAS, version 8.2, SAS Institute, Cary, NC; S-Plus, version 2000, Insightful, Seattle, Wash; and Stata, version 7.0, Stata, College Station, Tex) were used for data analysis.

\section{RESULTS}

At surgical histopathologic analysis, 83 (24.1\%) of 344 patients had evidence of ECE. Table 1 summarizes the distribution of the presurgical clinical variables, and Table 2 demonstrates the distribution of clinical and final pathologic stages. Performance characteristics for each variable are listed in Table 3. In the evaluation of ECE, endorectal MR imaging findings had sensitivity, specificity, positive predictive value, and negative predictive value of $42.2 \%, 95.4 \%$, $74.5 \%$, and $83.8 \%$, respectively (Table 3 ). Specificity of endorectal MR imaging findings was the highest among all variables, while sensitivity was second best after that of clinical stage of tumor.

\section{Table 1. Distribution of Presurgical Variables}

\begin{tabular}{|c|c|c|}
\hline Presurgical Variable & $\begin{array}{c}\text { No. of } \\
\text { Patients }\end{array}$ & Value \\
\hline PSA level (ng/mL) & 344 & $7.64 \pm 9.19(0.74-113.40)^{*}$ \\
\hline Gleason score & 344 & $5-10^{+}$ \\
\hline $\begin{array}{l}\text { Greatest percentage of cancer in all core } \\
\text { biopsy specimens }\end{array}$ & $322^{\ddagger}$ & $41 \pm 27(4-100)^{*}$ \\
\hline $\begin{array}{l}\text { Percentage of cancer-positive core } \\
\text { specimens in all core biopsy specimens }{ }^{\S}\end{array}$ & 344 & $33.71 \pm 27.29(1-100)^{*}$ \\
\hline \multicolumn{3}{|l|}{ PNI } \\
\hline Present & 48 & $14.0 \%$ \\
\hline Not present & 296 & $86.0 \%$ \\
\hline \multicolumn{3}{|l|}{ Clinical stagell } \\
\hline T1c & 195 & $56.7 \%$ \\
\hline $\mathrm{T} 2 \mathrm{a}$ and $\mathrm{T} 2 \mathrm{~b}$ & 116 & $33.7 \%$ \\
\hline $\mathrm{T} 2 \mathrm{C}$ & 33 & $9.6 \%$ \\
\hline $\mathrm{T} 3, \mathrm{~T} 4, \mathrm{~N}+$, and $\mathrm{M}+$ & 0 & $0 \%$ \\
\hline
\end{tabular}

* Data are the mean $\pm S D$. Data in parentheses are ranges.

+ Data are the range.

\# Data were not recorded in 22 patients.

$\S$ Values were calculated by dividing the number of cancer-positive core specimens by the total number of core biopsy specimens.

\|l Clinical stage of tumor was categorized according to TNM classifications. 
Table 2. Clinical and Final Pathologic Stage of Tumor

\begin{tabular}{|c|c|}
\hline Stage of Tumor & No. of Patients \\
\hline \multicolumn{2}{|l|}{ Clinical $^{*}$} \\
\hline $\mathrm{T} 1 \mathrm{C}$ & $195(56.7)$ \\
\hline $\mathrm{T} 2 \mathrm{a}$ and $\mathrm{T} 2 \mathrm{~b}$ & $116(33.7)$ \\
\hline $\mathrm{T} 2 \mathrm{C}$ & $33(9.6)$ \\
\hline $\mathrm{T} 3 \mathrm{a}$ and $\mathrm{T} 3 \mathrm{~b}$ & 0 \\
\hline $\mathrm{T} 3 \mathrm{c}$ & 0 \\
\hline T4 & 0 \\
\hline N1 & 0 \\
\hline M & 0 \\
\hline Final pathologic $^{+}$ & 116 \\
\hline TO & $2(0.6)$ \\
\hline T2a & 64 (18.6) \\
\hline $\mathrm{T} 2 \mathrm{~b}$ & $189(54.9)$ \\
\hline T3a & $57(16.6)$ \\
\hline T3b & $19(5.5)$ \\
\hline $\mathrm{T} 4$ & $13(3.8)$ \\
\hline N1 & $17(4.9)$ \\
\hline M & 0 \\
\hline
\end{tabular}

Note--Data in parentheses are percentages.

* Clinical stage of tumor was categorized according to TNM classifications (17).

${ }^{\dagger}$ Final pathologic stage of tumor was categorized according to TNM classifications (18).

Table 3. Performance Characteristics of Presurgical Variables at Select Cutoffs for Prediction of ECE

\begin{tabular}{lllll} 
Presurgical Variable & Sensitivity & Specificity & $\begin{array}{c}\text { Positive } \\
\text { Predictive } \\
\text { Value }\end{array}$ & $\begin{array}{c}\text { Negative } \\
\text { Predictive } \\
\text { Value }\end{array}$ \\
\hline Serum PSA level* & $27.7(23 / 83)$ & $87.4(228 / 261)$ & $41.1(23 / 56)$ & $79.2(228 / 288)$ \\
\hline $\begin{array}{l}\text { Gleason score } \\
\text { Clinical stage of tumor }\end{array}$ & $30.1(25 / 83)$ & $90.4(236 / 261)$ & $50.0(25 / 50)$ & $80.3(236 / 294)$ \\
$\begin{array}{l}\text { Greatest percentage of cancer in all core } \\
\text { biopsy specimens }\end{array}$ & $56.6(47 / 83)$ & $60.9(159 / 261)$ & $31.5(47 / 149)$ & $81.5(159 / 195)$ \\
\hline $\begin{array}{l}\text { Percentage of cancer-positive core speci } \\
\text { mens in all core biopsy specimens }\end{array}$ & $36.1(30 / 83)$ & $89.6(232 / 259)$ & $52.6(30 / 57)$ & $81.4(232 / 285)$ \\
\hline $\begin{array}{l}\text { PNI presence at needle biopsyll } \\
\text { Endorectal MR imaging findings }\end{array}$ & $35.0(28 / 80)$ & $81.0(196 / 242)$ & $37.8(28 / 74)$ & $79.0(196 / 248)$ \\
\hline & $25.3(21 / 83)$ & $89.7(234 / 261)$ & $43.8(21 / 48)$ & $79.1(234 / 296)$ \\
\hline
\end{tabular}

Note.-Data are percentages. Data in parentheses are the numbers used to calculate the percentages.

${ }^{*}$ Cutoff was $0-10.00 \mathrm{ng} / \mathrm{mL}$ versus $10.10 \mathrm{ng} / \mathrm{mL}$ or more.

+ Cutoff was $3+2,3+3$, and $3+4$ versus $3+5,4+3,4+4,4+5$, and $5+5$.

₹ Cutoff was T1c versus T2a, T2b, and T2c (categorized according to TNM classifications).

$\S$ Cutoff was $0 \%-60 \%$ versus more than $60 \%$.

\| Cutoff was present versus not present.

\# Cutoff was one to three versus four to five. 
Univariate analysis (Table 4) results showed that all variables were associated with ECE. At ROC univariate analysis, however (Fig 1), endorectal MR imaging findings had the largest area under the ROC curve, followed by greatest percentage of cancer in all core biopsy specimens and presurgical PSA level. Figure 1 demonstrates that at high specificity MR imaging findings had the best sensitivity, compared with all other variables. In a comparison of image interpretation between 216 patients who underwent both endorectal MR imaging and MR spectroscopic imaging (area under ROC curve $=0.777$ ) and 128 patients who underwent only endorectal MR imaging (area under ROC curve $=0.697)$, there was no significant difference $(P<.206)$.

\section{Table 4. Univariate Analysis: Assessment of Variables for Prediction of ECE}

\begin{tabular}{lcc} 
Predictor Variable* & P Value & Area under ROC Curve \\
\hline PSA level & $<.001$ & 0.670 \\
\hline Gleason score & $<.001$ & 0.603 \\
Clinical stage of tumor & $<.001$ & 0.588 \\
$\begin{array}{l}\text { Greatest percentage of cancer in all core } \\
\text { biopsy specimens }\end{array}$ & $<.001$ & 0.738 \\
$\begin{array}{l}\text { Percentage of cancer-positive core } \\
\text { specimens in all core biopsy specimens }\end{array}$ & $<.001$ & 0.635 \\
PNI presence & $<.001$ & 0.575 \\
Endorectal MR imaging findings & $<.001$ & 0.743
\end{tabular}

* Predictor variables were treated as continuous variables except for Gleason score (categorized as 3+2, 3+ 3, and 3+4 vs 3+5,4+3,4+4,4+5, and $5+5$ ) and clinical stage of tumor (categorized according to TNM classifications as T1c vs T2a, T2b, and T2c).

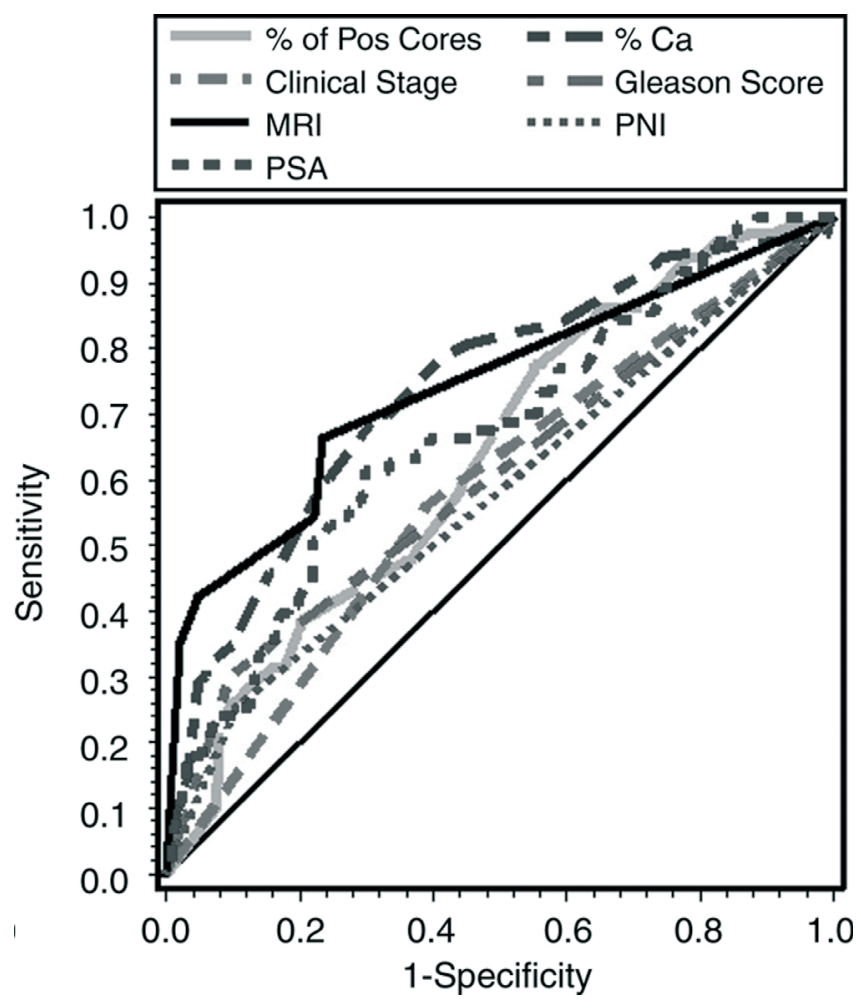

Figure 1. Graph shows comparison of ROC curves of seven variables for prediction of ECE. Area under ROC curve for MR imaging findings is 0.743 . Diagonal line indicates area under ROC curve of 0.500 (ie, no separation between patients with and patients without ECE). Predictor variables treated as continuous variables except for Gleason score (categorized as $3+2,3+3$, and $3+4$ vs $3+5,4+$ $3,4+4,4+5$, and $5+5)$ and clinical stage of cancer (categorized as T1c vs T2a, T2b, and T2c). \% Ca = greatest percentage of cancer in all core biopsy specimens, \% of Pos Cores = percentage of cancer-positive core specimens in all core biopsy specimens. 
With multivariate analysis, serum PSA level, greatest percentage of cancer in all core biopsy specimens, and endorectal MR imaging findings were significant predictors of $E C E(P=.001, P=.001$, and $P<.001$, respectively) (Figs 2, 3). The Gleason score had borderline significance as a predictor in both models, with and without endorectal MR imaging findings ( $P=.074$ and .057 , respectively) (Table 5). When the two models were compared, the area under the ROC curve of 0.838 for the model with clinical variables and endorectal MR imaging findings was greater than that of 0.772 for the model with clinical variables alone $(P=.022)$ (Table 5). The ROC curves for the models are shown in Figure 4.
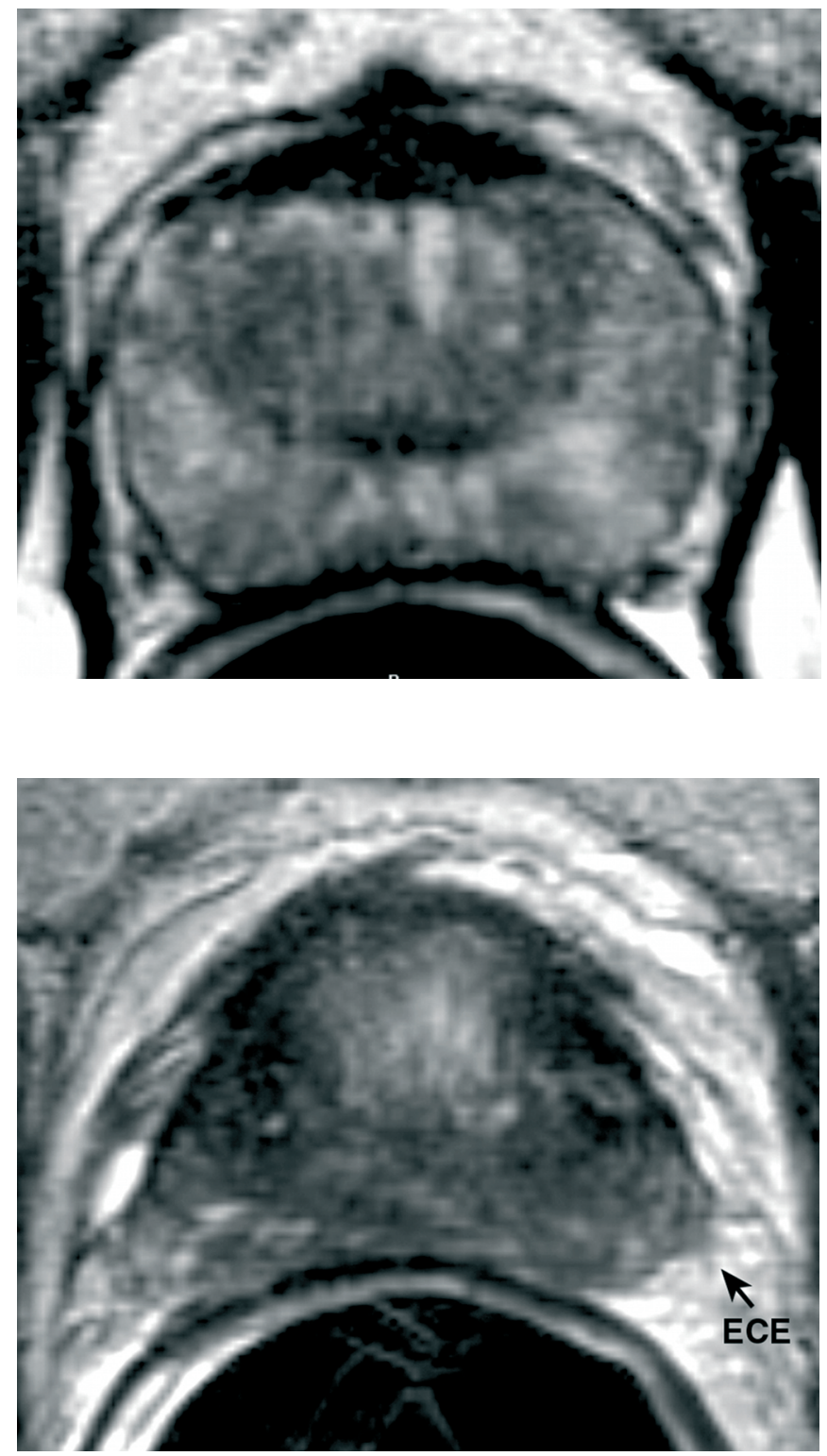

Figure 2. Transverse T2-weighted fast spin-echo 3mm-thick MR image $(5,266 / 121)$ in 61-year-old man with clinical stage T1c cancer shows no ECE. PSA level was $13.67 \mathrm{ng} / \mathrm{mL}$, sextant biopsy results indicated PNI in right middle area, and tumors with Gleason score of $4+$ 5 involved $50 \%$ of submitted tissue in both sides ( $14 \%$ of cores). Findings at final histopathologic analysis of step section indicated that tumor invaded prostate capsule but did not extend beyond it.
Figure 3. Transverse T2-weighted fast spin-echo 3-mmthick MR image $(5,933 / 121)$ in 61-year-old man with clinical stage T1c cancer and PSA level of $5.00 \mathrm{ng} / \mathrm{mL}$ shows ECE (arrow) at left posterior area. Sextant biopsy results were negative for PNI, and tumors with Gleason score of $4+4$ involved $30 \%$ of submitted tissue in the left side (43\% of cores). Findings at final histopathologic analysis of step section indicated focal ECE at left posterior area. 
Table 5. Multivariate Analysis: Assessment of Variables for Prediction of ECE

\begin{tabular}{|c|c|}
\hline Predictor Variable & $P$ Value \\
\hline \multicolumn{2}{|l|}{ Model without MR findings* } \\
\hline PSA level ${ }^{+}$ & $<.001$ \\
\hline Gleason score ${ }^{\ddagger}$ & .057 \\
\hline Clinical stage of tumor ${ }^{\S}$ & .323 \\
\hline $\begin{array}{l}\text { Greatest percentage of cancer in all score biopsy } \\
\text { specimens }^{\dagger}\end{array}$ & $<.001$ \\
\hline $\begin{array}{l}\text { Percentage of cancer-positive core specimens in all core } \\
\text { biopsy specimens }^{\dagger}\end{array}$ & .497 \\
\hline PNI presence & .987 \\
\hline \multicolumn{2}{|l|}{ Model with MR findings\| } \\
\hline PSA level ${ }^{\dagger}$ & .001 \\
\hline Gleason score ${ }^{\ddagger}$ & .074 \\
\hline Clinical stage of tumor ${ }^{5}$ & .523 \\
\hline $\begin{array}{l}\text { Greatest percentage of cancer in all core biopsy } \\
\text { specimens }^{\dagger}\end{array}$ & $<.001$ \\
\hline $\begin{array}{l}\text { Percentage of cancer-positive core specimens in all core } \\
\text { biopsy specimens }^{\dagger}\end{array}$ & .910 \\
\hline PNI presence & .914 \\
\hline ECE on MR images ${ }^{\dagger}$ & .001 \\
\hline
\end{tabular}

* Area under ROC curve was 0.772 , corrected with bootstrap method, for this model. + Variables analyzed as continuous variables.

\# Gleason score was obtained at biopsy (categorized as $3+2,3+3$, and $3+4$ vs $3+5,4+3,4+4,4+5$, and $5+5$ ).

$\S$ Clinical stage of tumor was categorized according to TNM classifications as T1c versus T2a, T2b, and T2c.

\| Area under ROC curve was 0.838 , corrected with bootstrap method, for this model. This model had a significantly larger area under the ROC curve $(0.838$ vs $0.772, P=.022)$.

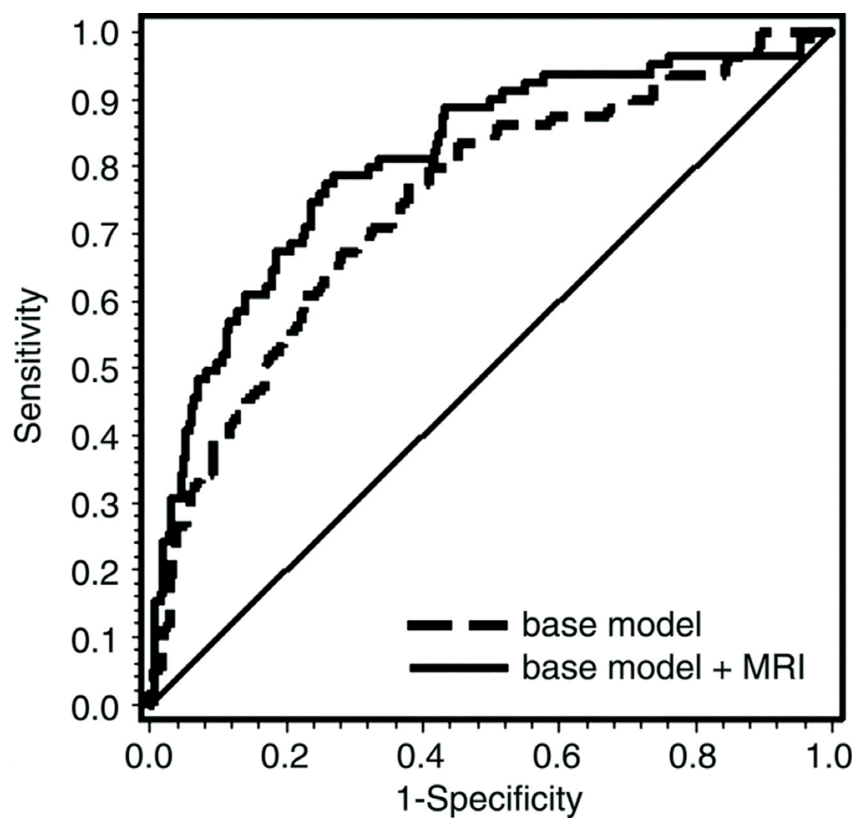

Figure 4. Graph shows ROC curves for comparison of models with and without MR imaging for prediction of ECE. Base model included the following variables: PSA level, Gleason score, clinical stage of tumor, greatest percentage of cancer in all core biopsy specimens, percentage of cancer-positive core specimens in all core biopsy specimens, and presence of PNI. Base model with endorectal MR imaging findings included MR imaging findings with all other variables. Predicted probability of both logistic regression models was bias corrected with jackknife method. Base model with endorectal MR imaging findings has greater area under ROC curve of 0.838 . (Color version available in chapter 18 ). 


\section{Discussion}

Pretreatment knowledge of ECE is important for both treatment selection and treatment planning. In treatment planning for surgery or radiation therapy, knowledge of ECE location is helpful for selection of the appropriate treatment approach. During the past years, studies in which the association between a number of clinical variables and the prediction of ECE was examined have been performed. The variables included serum PSA level, Gleason score, clinical stage of tumor, greatest percentage of cancer in all core biopsy specimens, percentage of cancer-positive core specimens in all core biopsy specimens, and presence of PNI $(4,19-21)$.

Findings in another investigation (22) and in our study indicate that serum PSA levels are associated with ECE with both univariate and multivariate analysis $(P \leq .001)$. In our study, Gleason score and clinical stage of tumor were significantly associated with ECE only with univariate analysis $(P<.001$ and $P=.005$, respectively). With multivariate analysis, Gleason score had borderline significance as a predictor in both models, with and without endorectal MR imaging findings ( $P=.074$ and .057 , respectively), while clinical stage of tumor was not significant. Similar findings were reported by Horiguchi et al (23). They demonstrated that MR imaging findings, along with Gleason score (categorized as 6 or 7) and PSA density, were significantly associated with ECE with multivariate logistic regression analysis $(P<.01)$.

Several features demonstrated at prostate biopsy were extensively examined as predictors of final pathologic stage (eg, greatest percentage of cancer in all core biopsy specimens, percentage of cancer-positive core specimens in all core biopsy specimens, and presence of PNI). Both Ravery et al (24) and Freedland et al (25) found percentage of cancer at core biopsy to be predictive of pathologic stage and risk of biochemical recurrence when they used multivariate analysis. Linson et al (26), however, found that only the percentage of cancer-positive core specimens was predictive of biochemical recurrence with multivariate analysis and therefore concluded that pursuing percentage of cancer in cancer-positive core specimens is an unnecessary and time-consuming task. In our study, with univariate analysis, both greatest percentage of cancer in all core biopsy specimens and percentage of cancer-positive core specimens in all core biopsy specimens were significant for the prediction of ECE ( $P<.001$ for both values). When multivariate analysis was used, however, only greatest percentage of cancer in all core biopsy specimens remained a significant predictor $(P<.001)$. This finding was not affected when results of endorectal MR imaging were integrated into the analysis.

Although there is controversy in regard to the value of PNI for the prediction of ECE, some urologists still use the finding of PNI in surgical planning (27). It has been suggested that the ipsilateral nerve bundle should be excised during radical prostatectomy when $\mathrm{PNI}$ is present $(27,28)$. Investigators in several studies $(20,29,30)$ address the utility of the finding of PNI in the prediction of ECE. Our results agree with the findings of Vargas et al (20) and of Egan and Bostwick (31) and show that the presence of PNI is a significant predictor of ECE with univariate analysis. In addition, progression of disease has also been examined.

In a regression analysis, Stone et al (32) found that the presence of PNI in a biopsy specimen correlated with lymph node metastasis at radical prostatectomy $(P=.04)$. D'Amico et al (33) and de la Taille et al (34) demonstrated that presence of PNI significantly correlates with PSA recurrence after radical prostatectomy. O'Malley et al (35), however, were not able to show a significant difference in PSA recurrence when they compared patients with PNI and patients without it who were treated with radical prostatectomy $(29,36)$. With our multivariate analysis, presence of PNI was not significant for the prediction of ECE ( $P=.987)$.

The quest for a better diagnostic test that can help differentiate between advanced and localized disease and assist a physician in treatment planning led to the evaluation of endorectal MR imaging in prostate cancer localization and staging. The routine use of MR imaging for presurgical evaluation of prostate cancer is controversial. The high incidence of the disease combined with the high cost of the test might burden the health care system with additional expenses unless its use prevents unnecessary surgery or aids in treatment planning and results in better outcomes (37). D'Amico et al (38) concluded that although MR imaging findings add significant predictive value (ie, prediction of the risk of developing biochemical failure following radical prostatectomy in $20 \%$ of patients), this result did not justify the routine use of the technique. Furthermore, endorectal MR imaging results demonstrated high interobserver variability, which limited the widespread use of the technique $(39,40)$. 
It has been suggested that treatment decisions should not be altered because of either endorectal MR imaging or transrectal ultrasonographic findings (41). In our study of the comparison of the value of endorectal MR imaging findings and clinical and histologic variables, endorectal MR imaging findings had a high negative predictive value and a high positive predictive value (83.8\% and $74.5 \%$, respectively). When assessed with univariate analysis, endorectal MR imaging findings demonstrated excellent prediction of ECE $(P<.001)$. In our study, the addition of MR spectroscopic imaging did not result in a significant improvement in image interpretation $(P<.206)$. Previously published data support the value of MR spectroscopic imaging in the detection and staging of prostate cancer (12). The incremental value of MR spectroscopic imaging was greater for the less experienced reader (12). Notably, MR spectroscopic technology in our institution was introduced in 2000, and there was a steep learning curve in the acquisition and interpretation of MR spectroscopic imaging data during the past 4 years.

With multivariate analysis, endorectal MR imaging findings, PSA level, and greatest percentage of cancer in all core biopsy specimens were all predictors of ECE $(P<.001)$. Because we recognized that results of such an analysis are insufficient for judgment of endorectal MR imaging findings as a new marker (16), we examined the incremental effect of endorectal MR imaging findings on predictive accuracy. When we compared the two models, one with and one without endorectal MR imaging findings, at multivariate analysis, we found that the area under the ROC curve of 0.838 for the model with endorectal MR imaging findings was significantly greater than that of 0.772 for the model without endorectal MR imaging findings $(P=.022)$. These data demonstrate the incremental value of endorectal MR imaging findings. In addition, endorectal MR imaging findings are spatially localized, and therefore, unlike clinical variables, they have the potential to allow tailored treatment modifications (12,42-44).

The strengths and limitations of our study are drawn from the fact that it was conducted in the routine clinical setting of the radiology department. Ten body MR imaging radiologists interpreted the images as part of their routine clinical assignments. There were differences in training and experience in prostate imaging among the readers, and their experience in interpretation of clinical MR images ranged from 4 to more than 15 years since fellowship. This may have resulted in a lack of data uniformity and in interobserver variability in the diagnosis of ECE. Since each image was interpreted by one reader, we could not assess the effect of interobserver variability on the accuracy of prediction of ECE at endorectal MR imaging. In a recently published study of tumor staging with meta-analysis at MR imaging, Engelbrecht et al (45) demonstrated considerable heterogeneity between endorectal MR imaging studies; they concluded that further studies are needed to establish the effect of the reader's experience, as well as the effect of the clinical information given to the reader, as they hypothesized that these factors may cause considerable differences in staging accuracy between the studies. A contrast material-enhanced study was not used in our protocol, though recent data suggest that such a study might improve accuracy in tumor staging (46).

With consideration of all these factors, our results show that endorectal MR imaging findings have remarkable strength in the prediction of ECE. Although further multicenter confirmatory study findings would be helpful, we suggest that endorectal MR imaging findings play an important role in the evaluation of prostate cancer and the prediction of ECE.

In conclusion, endorectal MR imaging findings are significant presurgical predictors of ECE in patients with prostate cancer, and they add incremental value to clinical variables.

\section{References}

1. Jemal A, Murray T, Samuels A, Ghafoor A, Ward E, Thun MJ. Cancer statistics, 2003. CA Cancer J Clin 2003; 53:5-26.

2. Polascik TJ, Oesterling JE, Partin AW. Prostate specific antigen: a decade of discovery-what we have learned and where we are going. J Urol 1999; 162:293-306.

3. Boring CC, Squires TS, Tong T. Cancer statistics, 1993. CA Cancer J Clin 1993; 43:7-26.

4. Partin AW, Mangold LA, Lamm DM, Walsh PC, Epstein JI, Pearson JD. Contemporary update of prostate cancer staging nomograms (Partin Tables) for the new millennium. Urology 2001; 58:843-848.

5. Kattan MW, Stapleton AM, Wheeler TM, Scardino PT. Evaluation of a nomogram used to predict the pathologic stage of clinically localized prostate carcinoma. Cancer 1997; 79:528-537.

6. Graefen M, Karakiewicz PI, Cagiannos I, et al. Validation study of the accuracy of a postoperative nomogram for recurrence after radical prostatectomy for localized prostate cancer. J Clin Oncol 2002; 20:951-956.

7. Mullerad M, Hricak H, Wang L, Chen HN, Kattan MW, Scardino PT. Prostate cancer: detection of extracapsular extension at MR imaging by genitourinary and general body radiologists. Radiology 2004; 232:140-146.

8. Bottomley PA. Selective volume method for performing localized MR spectroscopy. U.S. patent 44802281984. 
9. Star-Lack J, Nelson SJ, Kurhanewicz J, Huang LR, Vigneron DB. Improved water and lipid suppression for 3D PRESS CSI using RF band selective inversion with gradient dephasing (BASING). Magn Reson Med 1997; 38:311-321.

10. Brown TR, Kincaid BM, Ugurbil K. NMR chemical shift imaging in three dimensions. Proc Natl Acad Sci U S A 1982; 79:3523-3526.

11. Nelson S, Day MR, Carvajal L, et al. Methods for analysis of serial volume MRI and $1 \mathrm{H}$ MRS data for the assessment of response to therapy in patients with brain tumors (abstr) In: Proceedings of the Society of Magnetic Resonance in Medicine and the European Society of Magnetic Resonance in Medicine and Biology. Berkeley, Calif: Society of Magnetic Resonance in Medicine, 1995.

12. Yu KK, Scheidler J, Hricak H, et al. Prostate cancer: prediction of extracapsular extension with endorectal MR imaging and threedimensional proton MR spectroscopic imaging. Radiology 1999; 213:481-488.

13. Rubin MA, Bassily N, Sanda M, Montie J, Strawderman MS, Wojno K. Relationship and significance of greatest percentage of tumor and perineural invasion on needle biopsy in prostatic adenocarcinoma. Am J Surg Pathol 2000; 24:183-189.

14. Bismar TA, Lewis JS, Jr, Vollmer RT, Humphrey PA. Multiple measures of carcinoma extent versus perineural invasion in prostate needle biopsy tissue in prediction of pathologic stage in a screening population. Am J Surg Pathol 2003; 27:432-440.

15. Yossepowitch O, Sircar K, Scardino PT, et al. Bladder neck involvement in pathological stage pT4 radical prostatectomy specimens is not an independent prognostic factor. J Urol 2002; 168:2011-2015.

16. Kattan MW. Judging new markers by their ability to improve predictive accuracy. J Natl Cancer Inst 2003; 95:634-635.

17. American Joint Committee on Cancer. Manual for staging of cancer 4th ed. Philadel phia, Pa: Lippincott, 1992.

18. American Joint Committee on Cancer. AJCC cancer staging manual 5th ed. Philadel phia, Pa: Lippincott-Raven, 1997.

19. Graefen M, Karakiewicz PI, Cagiannos I, et al. International validation of a preoperative nomogram for prostate cancer recurrence after radical prostatectomy. J Clin Oncol 2002; 20:3206-3212.

20. Vargas SO, Jiroutek M, Welch WR, Nucci MR, D'Amico AV, Renshaw AA. Perineural invasion in prostate needle biopsy specimens: correlation with extraprostatic extension at resection. Am J Clin Pathol 1999; 111:223-228.

21. Roach M, 3rd, Chen A, Song J, Diaz A, Presti J, Jr, Carroll P. Pretreatment prostate-specific antigen and Gleason score predict the risk of extracapsular extension and the risk of failure following radiotherapy in patients with clinically localized prostate cancer. Semin Urol Oncol 2000; 18:108-114.

22. Paquette EL, Connelly RR, Sun L, Paquette LR, Moul JW. Predictors of extracapsular extension and positive margins in African American and white men. Urol Oncol 2003; 21:33-38.

23. Horiguchi A, Nakashima J, Horiguchi Y, et al. Prediction of extraprostatic cancer by prostate specific antigen density, endorectal MRI, and biopsy Gleason score in clinically localized prostate cancer. Prostate 2003; 56:23-29.

24. Ravery V, Chastang C, Toublanc M, Boccon-Gibod L, Delmas V. Percentage of cancer on biopsy cores accurately predicts extracapsular extension and biochemical relapse after radical prostatectomy for T1-T2 prostate cancer. Eur Urol 2000; 37:449-455.

25. Freedland SJ, Csathy GS, Dorey F, Aronson WJ. Percent prostate needle biopsy tissue with cancer is more predictive of biochemical failure or adverse pathology after radical prostatectomy than prostate specific antigen or Gleason score. J Urol 2002; 167:516-520.

26. Linson PW, Lee AK, Doytchinova T, et al. Percentage of core lengths involved with prostate cancer: does it add to the percentage of positive prostate biopsies in predicting postoperative prostate-specific antigen outcome for men with intermediate-risk prostate cancer? Urology 2002; 59:704-708.

27. Epstein Jl, Potter SR. The pathological interpretation and significance of prostate needle biopsy findings: implications and current controversies. J Urol 2001; 166:402-410.

28. Bastacky SI, Walsh PC, Epstein Jl. Relationship between perineural tumor invasion on needle biopsy and radical prostatectomy capsular penetration in clinical stage B adenocarcinoma of the prostate. Am J Surg Pathol 1993; 17:336-341.

29. Bastacky SS, Epstein Jl. The sensitivity and specificity of perineural invasion on needle-biopsy of prostate-cancer in predicting capsular penetration at radical prostatectomy (abstr). Lab Invest 1991; 64:A43.

30. de la Taille A, Katz A, Bagiella E, Olsson CA, O'Toole KM, Rubin MA. Perineural invasion on prostate needle biopsy: an independent predictor of final pathologic stage. Urology 1999; 54:1039-1043.

31. Egan AJ, Bostwick DG. Prediction of extraprostatic extension of prostate cancer based on needle biopsy findings: perineural invasion lacks significance on multivariate analysis. Am J Surg Pathol 1997; 21:1496-1500.

32. Stone NN, Stock RG, Parikh D, Yeghiayan P, Unger P. Perineural invasion and seminal vesicle involvement predict pelvic lymph node metastasis in men with localized carcinoma of the prostate. J Urol 1998; 160:1722-1726.

33. D'Amico AV, Wu Y, Chen MH, Nash M, Renshaw AA, Richie JP. Perineural invasion as a predictor of biochemical outcome following radical prostatectomy for select men with clinically localized prostate cancer. J Urol 2001; 165:126-129.

34. de la Taille A, Rubin MA, Bagiella E, et al. Can perineural invasion on prostate needle biopsy predict prostate specific antigen recurrence after radical prostatectomy? J Urol 1999; 162:103-106.

35. O'Malley KJ, Pound CR, Walsh PC, Epstein JI, Partin AW. Influence of biopsy perineural invasion on long-term biochemical disease-free survival after radical prostatectomy. Urology 2002; 59:85-90.

36. D'Amico AV. Perineural invasion as a predictor of PSA outcome following local therapy for patients with clinically localized prostate cancer. Cancer J 2001; 7:375-376.

37. Penson DF, Litwin MS, Aaronson NK. Health related quality of life in men with prostate cancer. J Urol 2003; 169:1653-1661.

38. D'Amico AV, Whittington R, Malkowicz B, et al. Endorectal magnetic resonance imaging as a predictor of biochemical outcome after radical prostatectomy in men with clinically localized prostate cancer. J Urol 2000; 164:759-763.

39. Tempany CM. MR staging of prostate cancer: how we can improve our accuracy with decisions aids and optimal techniques. Magn Reson Imaging Clin N Am 1996; 4:519-532.

40. Tempany CM, Zhou X, Zerhouni EA, et al. Staging of prostate cancer: results of Radiology Diagnostic Oncology Group project comparison of three MR imaging techniques. Radiology 1994; 192:47-54.

41. May F, Treumann T, Dettmar P, Hartung R, Breul J. Limited value of endorectal magnetic resonance imaging and transrectal ultrasonography in the staging of clinically localized prostate cancer. BJU Int 2001; 87:66-69.

42. Cornud F, Flam T, Chauveinc L, et al. Extraprostatic spread of clinically localized prostate cancer: factors predictive of pT3 tumor and of positive endorectal MR imaging examination results. Radiology 2002; 224:203-210.

43. D'Amico AV, Cormack RA, Tempany CM. MRI-guided diagnosis and treatment of prostate cancer. N Engl J Med 2001; 344:776-777.

44. Scheidler J, Hricak H, Vigneron DB, et al. Prostate cancer: localization with three-dimensional proton MR spectroscopic imagingclinicopathologic study. Radiology 1999; 213:473-480. 


\section{B}

45. Engelbrecht MR, Jager GJ, Laheij RJ, Verbeek AL, van Lier HJ, Barentsz JO. Local staging of prostate cancer using magnetic resonance imaging: a meta-analysis. Eur Radiol 2002; 12:2294-2302.

46. Ogura K, Maekawa S, Okubo K, et al. Dynamic endorectal magnetic resonance imaging for local staging and detection of neurovascular bundle involvement of prostate cancer: correlation with histopathologic results. Urology 2001; 57:721-726. 


\section{Prostate Cancer: Detection of Extracapsular Extension by Genitourinary and General Body Radiologists at MR Imaging}




\title{
Prostate Cancer: Detection of Extracapsular Extension by Genitourinary and General Body Radiologists at MR Imaging
}

\author{
Supported by National Institutes of Health grant R01 CA76423.
}

Michael Mullerad, Hedvig Hricak, Liang Wang, Hui-Ni Chen, Michael W. Kattan, and Peter T. Scardino

From the Departments of Urology (M.M., M.W.K., P.T.S.) and Radiology (H.H., L.W., H.N.C.), Memorial Sloan-Kettering Cancer Center, 1275 York Ave, New York, NY 10021. Received August 6, 2003; revision requested September 18; revision received December 19; accepted January 13, 2004.

\section{Abstract}

Purpose: To determine whether predictive value of endorectal magnetic resonance (MR) imaging findings in detection of prostate cancer extracapsular extension (ECE) is significantly affected by the reader's subspecialty experience.

Materials and Methods: In this cohort study, 344 consecutive patients with biopsy-proved prostate cancer underwent endorectal MR imaging followed by surgery. Likelihood of ECE described in MR imaging reports was compared with clinical predictor variables. ECE was determined from the final pathologic report on specimens resected at surgery. Readers of MR images were classified into genitourinary MR imaging radiologists $(n=4)$ and general body MR imaging radiologists $(n=6)$. For data analysis, Wilcoxon rank sum and 2 tests, as well as receiver operating characteristic (ROC) curves and univariate and multivariate logistic regression analyses, were used. A difference with $\mathrm{P}<.05$ was considered significant.

Results: Univariate analysis results demonstrated that all predictors except clinical stage were significantly associated with detection of ECE in both groups of readers $(P<.05)$. In the genitourinary MR imaging radiologist group of patients, area under the ROC curve for endorectal MR imaging findings (0.833) was larger than areas under the curves for all other predictors (0.566-0.701). In the general body MR imaging radiologist group of patients, area under the ROC curve for endorectal MR imaging findings (0.646) was not larger than areas under the curves for all other predictors (0.582-0.793). Results of multivariate analysis of two models, one with all predictors and another with all predictors except endorectal MR imaging findings, demonstrated a significant increase in area under the ROC curve with endorectal MR images interpreted by genitourinary MR imaging radiologists $(P=.019$ and .31 , respectively).

Concusion: Endorectal MR imaging findings are significant predictors for detection of ECE when MR images are interpreted by genitourinary radiologists experienced with MR imaging of the prostate.

() RSNA, 2004 


\section{INTRODUCTION}

$\mathbf{T}$ he increase in early detection of prostate cancer has created new challenges in diagnosis and treatment. Today, the treatment of prostate cancer is customized to the status of the disease in each patient. As a result, a precise determination of disease extension and of risk of cancer recurrence must be made before treatment can be applied.

Presurgical variables commonly used for the prediction of pathologic stage of prostate cancer are prostate-specific antigen (PSA) level, Gleason score, and clinical tumor stage (evaluated with findings at digital rectal examination and transrectal ultrasonography) (1-3). Nomographic models have been created to help physicians predict the final pathologic stage of the tumor and the risk of cancer recurrence within 5 years on the basis of the patient's individual parameters (1-3). These nomograms provide a statistical prediction but lack anatomic data that can assist in intervention aimed to control local disease. Therefore, the quest for a better diagnostic test that would enable physicians to differentiate between advanced and localized disease and improve treatment planning led to the evaluation of the role of endorectal magnetic resonance (MR) imaging in the localization and the staging of prostate cancer. Researchers in early studies reported inter- and intraobserver variability, as well as inconsistency in the accuracy of diagnoses determined with MR imaging findings (4-8). Data in these reports in part may explain why 20 years after the introduction of MR imaging to clinical practice, referring physicians tend to regard this modality as having limited value for evaluation of patients with prostate cancer (9).

The aim of our study was to determine whether the predictive value of endorectal MR imaging findings in the detection of ECE is significantly affected by the reader's subspecialty experience.

\section{Materials and Methods}

\section{Patients}

Between May 1998 and January 2003, patients referred for endorectal MR imaging of the prostate prior to radical prostatectomy were enrolled in the study; all had biopsy-proved prostate cancer. Mean patient age was 57.5 years (range, 32-74 years). Patients who received neoadjuvant hormonal or radiation therapy before surgery were excluded from the study. Overall, 344 patients were included in the data analysis; 216 of these patients underwent endorectal MR imaging and MR spectroscopic imaging. The same patient population was used for the study of Wang et al (10). The study was approved by the institutional review board, and each patient signed an informed consent form before enrollment.

\section{Imaging and Image Interpretation}

Endorectal MR imaging and hydrogen 1 MR spectroscopic imaging were performed with a 1.5-T whole-body MR imaging unit (Signa Horizon; GE Medical Systems, Milwaukee, Wis). The examination was performed with patients in the supine position. A body coil was used for excitation, and a pelvic phased-array coil (GE Medical Systems) combined with a commercially available balloon-covered expandable endorectal coil (Medrad, Pittsburgh, Pa) was used for signal reception. Transverse T1-weighted and spin-echo MR images were obtained from the aortic bifurcation to the symphysis pubis with the following parameters: repetition time msec/echo time msec, 700/8; section thickness, $5 \mathrm{~mm}$; intersection gap, $1 \mathrm{~mm}$; field of view, $24 \mathrm{~cm}$; matrix, $256 \times 192$; and frequency direction, transverse (to prevent obstruction of the pelvic node from endorectal coil motion artifact). One signal was acquired.

Transverse and coronal thin-section high-spatial-resolution T2-weighted fast spin-echo MR images of the prostate and seminal vesicle were obtained with the following parameters: 5,000/96 (effective); echo train length, 16; section thickness, 3 mm; intersection gap, 0 mm; field of view, 14 cm; matrix, 256 × 192; frequency direction, anteroposterior (to prevent obstruction of the prostate from endorectal coil motion artifact); and number of signals acquired, three.

\section{${ }^{1}$ Abbreviations}

$\mathrm{ECE}=$ extracapsular extension, $\mathrm{PNI}=$ perineural invasion, $\mathrm{PSA}=$ prostate-specific antigen, $\mathrm{ROC}=$ receiver operating characteristic 
MR spectroscopic imaging was performed by using point-resolved spectroscopic voxel excitation (11), band-selective inversion with gradient dephasing water and lipid suppression (12), and spatial encoding with chemical shift imaging (13) at 6.25-mm resolution in all three dimensions (left-right, anterior-posterior, superior-inferior dimensions). Parameters were 1,000/130, and imaging time was 17 minutes.

Data processing was performed at a workstation (Sun Ultra 10; Sun Microsystems, Mountain View, Calif) and included 2-Hz Lorentzian spectral apodization; four-dimensional Fourier transform; and automated frequency, phase, and baseline correction (14). Spectral data were zero filled to 3.1-mm resolution in the superior-inferior dimension and overlaid on corresponding transverse T2-weighted MR images. Peak areas were calculated by using numeric integration. To provide a noise measurement, we calculated the SD of the MR signal intensity in a region of the spectrum containing only noise. Metabolite peak areas were then normalized with respect to the noise SD to yield an approximate signal-to-noise ratio.

MR images were interpreted by 10 MR imaging radiologists (four genitourinary MR imaging radiologists and six general body MR imaging radiologists), including one of the authors (H.H.), during their regular clinical assignment to the MR imaging service. All readers were trained in body MR imaging (seven had an MR imaging fellowship and the others had been involved with MR imaging since it was introduced to clinical practice), but the genitourinary MR imaging radiologists had extensive experience in genitourinary imaging and more than 3 years of experience in prostate imaging. They regularly attended urology grand rounds and prostate tumor board conferences and were involved in the field academically. Experience in interpretation of MR images ranged from 4 to 15 years since fellowship for the genitourinary MR imaging radiologists and from 6 to 10 years since fellowship for the general body MR imaging radiologists.

In 344 patients, MR images in 163 patients were evaluated by the genitourinary MR imaging radiologists and those in the remaining 181 patients were evaluated by the general body MR imaging radiologists. There was no initial meeting or training to establish the diagnostic criteria for ECE on endorectal MR images. Rather, radiologists made their determinations on the basis of their own continuing medical training and knowledge of previously described MR imaging features of ECE. The diagnostic criteria used by the radiologists included irregular capsular bulge, periprostatic fat infiltration, obliteration of the rectoprostatic angle, and asymmetry or direct involvement of the neurovascular bundles (15). All readers had access to MR spectroscopic imaging data when available, but such data were not always used. In addition, all readers had access to the patients' medical records, including PSA level and biopsy findings. On the basis of the radiologists' written reports, one observer (L.W.) retrospectively scored the likelihood of ECE with a five-point scale as follows: score 1, no ECE; score 2, probably no ECE (cannot be ruled out though there is no clear evidence of it); score 3, possible ECE (a lesion is suspected of demonstrating ECE); score 4, probable ECE (a lesion is highly suspected of demonstrating ECE); score 5, definite ECE.

\section{Clinical Data and Reference Standards}

Presurgical clinical data, as well as pathologic reports at biopsy were evaluated for PSA level, Gleason score, greatest percentage of cancer in all core biopsy specimens, percentage of cancer-positive core specimens in all core biopsy specimens (the number of cancer-positive core specimens divided by the total number of core biopsy specimens), presence of perineural invasion (PNI), and clinical stage of tumor. The greatest percentage of cancer was determined in each patient by examining each core biopsy specimen and dividing the length of the core specimen tissue with cancer by the whole core specimen length; the core specimen with the highest percentage of cancer defined the patient's greatest percentage of cancer. This parameter has been previously described by Rubin et al (16) and Bismar et al (17). Results from the final pathologic report following surgery were used to determine ECE. ECE was defined as the presence of tumor cells outside the prostate capsule. All 344 patients had undergone radical prostatectomy.

Specimens removed at radical prostatectomy were examined by the institutional pathology department, with methods previously described by Yossepowitch et al (18). Biopsy was performed at our institution, or biopsy findings were reviewed by our pathologists.

\section{Statistical Analysis}

The Wilcoxon rank sum test was used to compare the presurgical continuous predictor variables of the two groups of patients (one composed of patients whose MR images were interpreted by general body MR imaging radiologists and the other composed of patients whose MR images were interpreted by genitourinary MR imaging radi- 
ologists). Hereafter, these groups will be referred to as the general body radiologist group and the genitourinary radiologist group, respectively. The 2 test was used to evaluate the differences between the categorical predictor variables of the two groups, as well as to evaluate the difference between the percentages of the two groups in regard to the number of final pathologic specimens that showed ECE. Predictor variables that we tested included PSA level, Gleason score, greatest percentage of cancer in all core biopsy specimens, percentage of cancer-positive core specimens in all core biopsy specimens, presence of $\mathrm{PNI}$, and clinical stage of tumor, as well as the probability of ECE as scored retrospectively on the basis of findings in endorectal MR imaging reports. We calculated the area under the receiver operating characteristic (ROC) curve for each variable in the univariate regression analysis for the prediction of ECE. To determine the predictive value of endorectal MR imaging findings, we used multivariate regression analysis. Two models were constructed: One model included all the clinical variables with endorectal MR imaging findings, and the other model included only the clinical variables without endorectal MR imaging findings. Jackknife-predicted probabilities of ECE for models with and without endorectal MR imaging findings were used to construct the ROC curves. $P$ values were bias corrected by using the bootstrap method. A difference with $P<.05$ was considered significant.

\section{RESULTS}

Tables 1-4 summarize the distribution of clinical variables between the genitourinary radiologist group and the general body radiologist group, as well as the prevalence of ECE in final pathologic specimens. Eighty-three (24.1\%) patients with prostate cancer had evidence of ECE in the final pathologic specimen. There were no significant differences in predictor variables or in the prevalence of ECE between the two groups.

\section{Table 1. Distribution of Predictor Variables according to Group}

\begin{tabular}{|c|c|c|}
\hline Predictor Variable & $\begin{array}{c}\text { Genitourinary } \\
\text { Radiologist Group }\end{array}$ & $\begin{array}{c}\text { General Body } \\
\text { Radiologist Group }\end{array}$ \\
\hline \multicolumn{3}{|l|}{ PSA level } \\
\hline No. of patients & 163 & 181 \\
\hline Mean $\pm S D^{*}$ & $7.76 \pm 9.81$ & $7.54 \pm 8.61$ \\
\hline Range* & $1.23-113.40$ & $0.74-78.44$ \\
\hline \multicolumn{3}{|l|}{ Gleason score } \\
\hline No. of patients & 163 & 181 \\
\hline Mean $\pm S D$ & $6.44 \pm 0.66$ & $6.47 \pm 0.76$ \\
\hline Range & $6-9$ & $5-10$ \\
\hline \multicolumn{3}{|c|}{ Greatest percentage of cancert } \\
\hline No. of patients & 163 & 179 \\
\hline Mean \pm SD & $34.55 \pm 27.39$ & $32.95 \pm 27.26$ \\
\hline Range & $1.00-98.00$ & $1.00-100.00$ \\
\hline \multicolumn{3}{|c|}{$\begin{array}{l}\text { Percentage of cancer-positive core biopsy } \\
\text { specimens } \neq\end{array}$} \\
\hline No. of patients & 157 & 165 \\
\hline Mean $\pm S D$ & $40 \pm 25$ & $42 \pm 28$ \\
\hline Range & $4-100$ & $6-100$ \\
\hline
\end{tabular}

Note.-P values in both groups were as follows: PSA level, .95; Gleason score, .96; greatest percentage of cancer, .40; and percentage of cancerpositive core specimens, 73

* Measured in nanograms per milliliter.

+ In all core biopsy specimens. Data missing for two patients.

${ }^{\ddagger}$ In all core biopsy specimens. Data missing for 22 patients. 
Table 2. Clinical Stage of Tumor according to Group

\begin{tabular}{lccc} 
Clinical Stage of Tumor* & $\begin{array}{c}\text { Genitourinary } \\
\text { Radiologist Group }\end{array}$ & $\begin{array}{c}\text { General Body } \\
\text { Radiologist Group }\end{array}$ & Total \\
\hline T1c & $94(57.7)$ & $101(55.8)$ & 195 \\
\hline T2a & $43(26.4)$ & $36(19.9)$ & 79 \\
T2b & $14(8.6)$ & $23(12.7)$ & 37 \\
\hline T2c & $12(7.4)$ & $21(11.6)$ & 33 \\
Overall & 163 & 181 & 344
\end{tabular}

Note.-Data are numbers of patients. Data in parentheses are percentages. Percentages were rounded. $P=.20,2$ test.

${ }^{*}$ According to TNM classifications.

Table 3. Presence of PNI at Biopsy according to Group

\begin{tabular}{lccc} 
PNI & $\begin{array}{c}\text { Genitourinary } \\
\text { Radiologist Group }\end{array}$ & $\begin{array}{c}\text { General Body } \\
\text { Radiologist Group }\end{array}$ & Total \\
\hline Not present & $138(84.7)$ & $158(87.3)$ & 296 \\
\hline Present & $25(15.3)$ & $23(12.7)$ & 48 \\
Overall & 163 & 181 & 344
\end{tabular}

Note.-Data are numbers of patients. Data in parentheses are percentages. Percentages were rounded. $P=.48,2$ test.

Table 4. Prevalence of ECE in Final Pathologic Specimens according to Group

\begin{tabular}{lccc} 
ECE & $\begin{array}{c}\text { Genitourinary } \\
\text { Radiologist Group }\end{array}$ & $\begin{array}{c}\text { General Body } \\
\text { Radiologist Group }\end{array}$ & Total \\
\hline Not present & $119(73.0)$ & $142(78.4)$ & 261 \\
Present & $44(27.0)$ & $39(21.5)$ & 83 \\
Overall & 163 & 181 & 344
\end{tabular}

Note.-Data are numbers of patients. Data in parentheses are percentages. Percentages were rounded. $P=.24,2$ test.

In Figure 1, the ROC curve for endorectal MR imaging findings in the genitourinary radiologist group demonstrates a combination of sensitivity and specificity that is superior to that of every other variable in the patient population. In the general body radiologist group, however, the ROC curve for endorectal MR imaging findings demonstrates a combination of sensitivity and specificity that is similar to those of the other predictors. With univariate analysis (Table 5), all variables tested were significantly associated with findings of ECE, with the exception of clinical stage of tumor in the genitourinary radiologist group. 


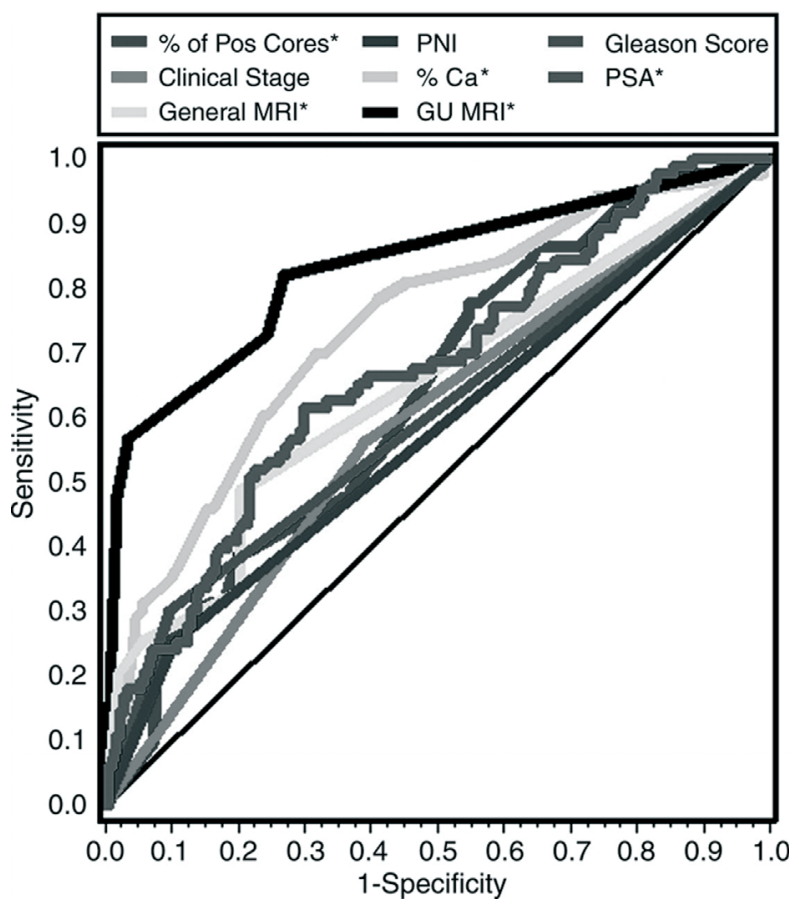

Figure 1. Graph shows ROC curves of univariate analysis results for all predictors of ECE. Predictors analyzed as continuous variables are indicated $(*)$. Gleason score was categorized as $3+2$ and $3+3$ versus $3+4,4+3,3+5,4+4,4+5$, and $5+5$. Clinical stage of tumor was categorized according to TNM classifications as T1c versus T2a, T2b, and T2c. Diagonal line indicates area under ROC curve of 0.500. General MRI = endorectal MR imaging findings for general body radiologist group, \% Ca = greatest percentage of cancer in all core biopsy specimens, GU $M R I=$ endorectal MR imaging findings for genitourinary radiologist group, \% of Pos Cores = percentage of cancer-positive core specimens in all core biopsy specimens.

(Color version available in chapter 18).

Table 5. Univariate Analysis: Assessment of Variables for Prediction of ECE according to Group

\begin{tabular}{|c|c|c|}
\hline Predictor Variable & PValue & Area under ROC Curve \\
\hline \multicolumn{3}{|l|}{ Genitourinary radiologist group } \\
\hline PSA level* & .002 & 0.701 \\
\hline Gleason score $^{t}$ & $<.001$ & 0.688 \\
\hline $\begin{array}{l}\text { Greatest percentage of cancer in all core } \\
\text { biopsy specimens* }\end{array}$ & $<.001$ & 0.681 \\
\hline $\begin{array}{l}\text { Percentage of cancer-positive core } \\
\text { specimens in all core biopsy specimens* }\end{array}$ & .012 & 0.639 \\
\hline PNI presence & $<.042$ & 0.566 \\
\hline Clinical stage of tumor ${ }^{\ddagger}$ & $<.120$ & 0.568 \\
\hline MR imaging findings* & $<.001$ & 0.833 \\
\hline \multicolumn{3}{|l|}{ General body radiologist group } \\
\hline PSA level* & .021 & 0.645 \\
\hline Gleason score $^{t}$ & $<.001$ & 0.683 \\
\hline $\begin{array}{l}\text { Greatest percentage of cancer in all core } \\
\text { biopsy specimens* }\end{array}$ & $<.001$ & 0.793 \\
\hline $\begin{array}{l}\text { Percentage of cancer-positive core } \\
\text { specimens in all core biopsy specimens* }\end{array}$ & .016 & 0.634 \\
\hline PNI presence & .009 & 0.582 \\
\hline Clinical stage of tumor ${ }^{\ddagger}$ & .015 & 0.611 \\
\hline MR imaging findings* & $<.001$ & 0.646 \\
\hline
\end{tabular}

* Predictor analyzed as continuous variable.

+ Gleason score was categorized as $3+$ 2 and $3+3$ versus $3+4,4+3,3+5,4+$ $4,4+5$, and $5+5$.

₹ Clinical stage of tumor was categorized as T1c versus $\mathrm{T} 2 \mathrm{a}, \mathrm{T} 2 \mathrm{~b}$, and $\mathrm{T} 2 \mathrm{c}$ according to TNM classifications. 
With multivariate analysis (Table 6), we examined the accuracy of prediction of ECE of our two models of variables (one with and one without endorectal MR imaging findings). Both models were used for both the genitourinary radiologist and the general body radiologist groups. The area under the ROC curve was calculated for each model.

Table 6. Multivariate Analysis: Assessment of Variables for Prediction of ECE according to Group and Model

\begin{tabular}{|c|c|}
\hline Predictor Variable & $P$ Value \\
\hline \multicolumn{2}{|c|}{ Genitourinary radiologist Group Model with imaging findings } \\
\hline PSA level* & .032 \\
\hline Gleason score $^{\dagger}$ & .097 \\
\hline $\begin{array}{l}\text { Greatest percentage of cancer in all core } \\
\text { biopsy specimens* }\end{array}$ & .476 \\
\hline $\begin{array}{l}\text { Percentage of cancer-positive core } \\
\text { specimens in all core biopsy specimens* }\end{array}$ & .862 \\
\hline PNI presence & .915 \\
\hline Clinical stage of tumor ${ }^{\ddagger}$ & .795 \\
\hline MR imaging findings & $<.001$ \\
\hline \multicolumn{2}{|l|}{ Model without MR imaging findings } \\
\hline PSA level* & .009 \\
\hline Gleason score $^{\dagger}$ & .016 \\
\hline $\begin{array}{l}\text { Greatest percentage of cancer in all core } \\
\text { biopsy specimens* }\end{array}$ & .032 \\
\hline $\begin{array}{l}\text { Percentage of cancer-positive core } \\
\text { specimens in all core biopsy specimens* }\end{array}$ & .412 \\
\hline PNI presence & .863 \\
\hline Clinical stage of tumor ${ }^{\ddagger}$ & .729 \\
\hline \multicolumn{2}{|c|}{ General body radiologist group model with MR imaging findings } \\
\hline PSA level & .032 \\
\hline Gleason score $^{\dagger}$ & .206 \\
\hline $\begin{array}{l}\text { Greatest percentage of cancer in all core } \\
\text { biopsy specimens* }\end{array}$ & .001 \\
\hline $\begin{array}{l}\text { Percentage of cancer-positive core } \\
\text { specimens in all core biopsy specimens* }\end{array}$ & .446 \\
\hline PNI presence & .929 \\
\hline Clinical stage of tumor & .281 \\
\hline MR imaging findings & .002 \\
\hline \multicolumn{2}{|l|}{ Model without MR imaging findings } \\
\hline PSA level & .048 \\
\hline Gleason score $^{\dagger}$ & .188 \\
\hline $\begin{array}{l}\text { Greatest percentage of cancer in all core } \\
\text { biopsy specimens* }\end{array}$ & .002 \\
\hline $\begin{array}{l}\text { Percentage of cancer-positive core } \\
\text { specimens in all core biopsy specimens* }\end{array}$ & .682 \\
\hline PNI presence & .918 \\
\hline Clinical stage of tumor ${ }^{\ddagger}$ & .277 \\
\hline
\end{tabular}

Note.-The model with endorectal MRimaging findings (area under ROC curve $=0.854$ ) had a significantly larger area under the ROC curve than did the model without these findings (area under ROC curve $=0.760$ ) for the genitourinary radiologist group $(P=.019)$. The area under the ROC curve for the model with MR imaging findings was 0.813 and that for the model without these findings was 0.788 for the general body radiologist group $(P=.31)$. All areas under the ROC curves were corrected with the bootstrap method.

* Predictor variable analyzed as continuous variable.

† Gleason score at biopsy was categorized as $3+2$ and $3+3$ versus $3+4,4+3,3+5,4+$ $4,4+5$, and $5+5$.

\# Clinical stage of tumor was categorized as $\mathrm{T} 1 \mathrm{c}$ versus $\mathrm{T} 2 \mathrm{a}, \mathrm{T} 2 \mathrm{~b}$, and $\mathrm{T} 2 \mathrm{c}$ according to TNM classifications.

In the genitourinary radiologist group, in the model without MR imaging findings, $P S A$ level $(P=.009)$, Gleason score $(P=.016)$, and greatest percentage of cancer in all core biopsy specimens $(P=.032)$ were all significant predictors of ECE. When included in the model, endorectal MR imaging findings were significant predictors of $E C E(P<.001)$ (Table 6). Furthermore, the model with endorectal MR imaging findings had a greater area under the ROC curve than did the model without endorectal MR imaging findings (area under the ROC curve $=0.854$ and 0.760 , respectively; $\mathrm{P}=$.019) (Fig 2a). 


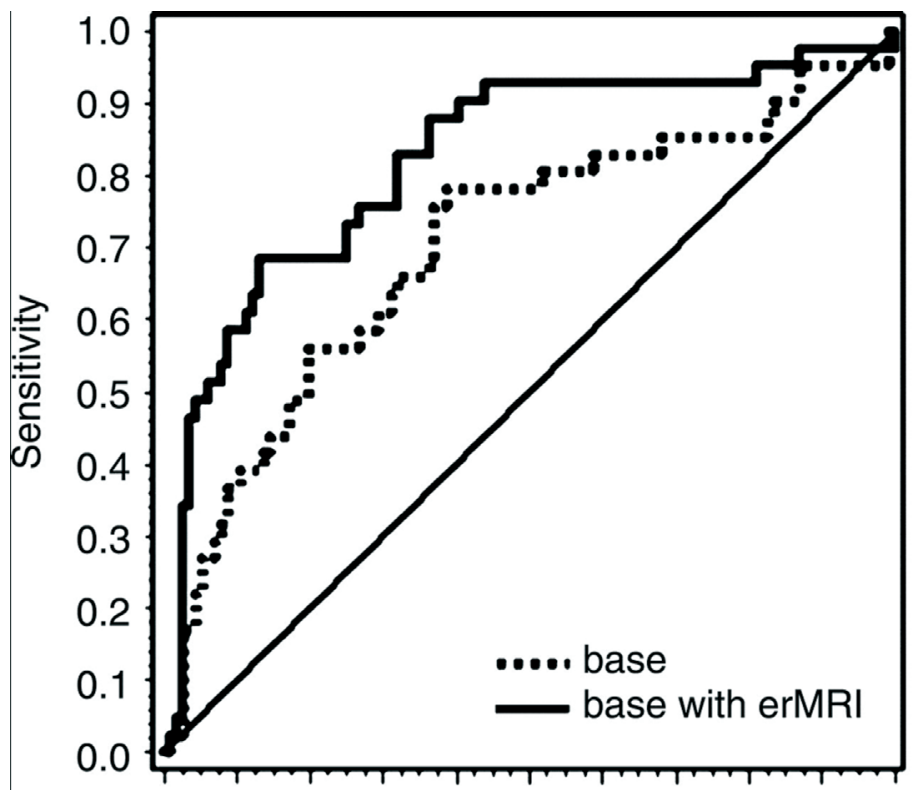

$\begin{array}{lllllllllll}0.0 & 0.1 & 0.2 & 0.3 & 0.4 & 0.5 & 0.6 & 0.7 & 0.8 & 0.9 & 1.0\end{array}$

\section{1-Specificity}

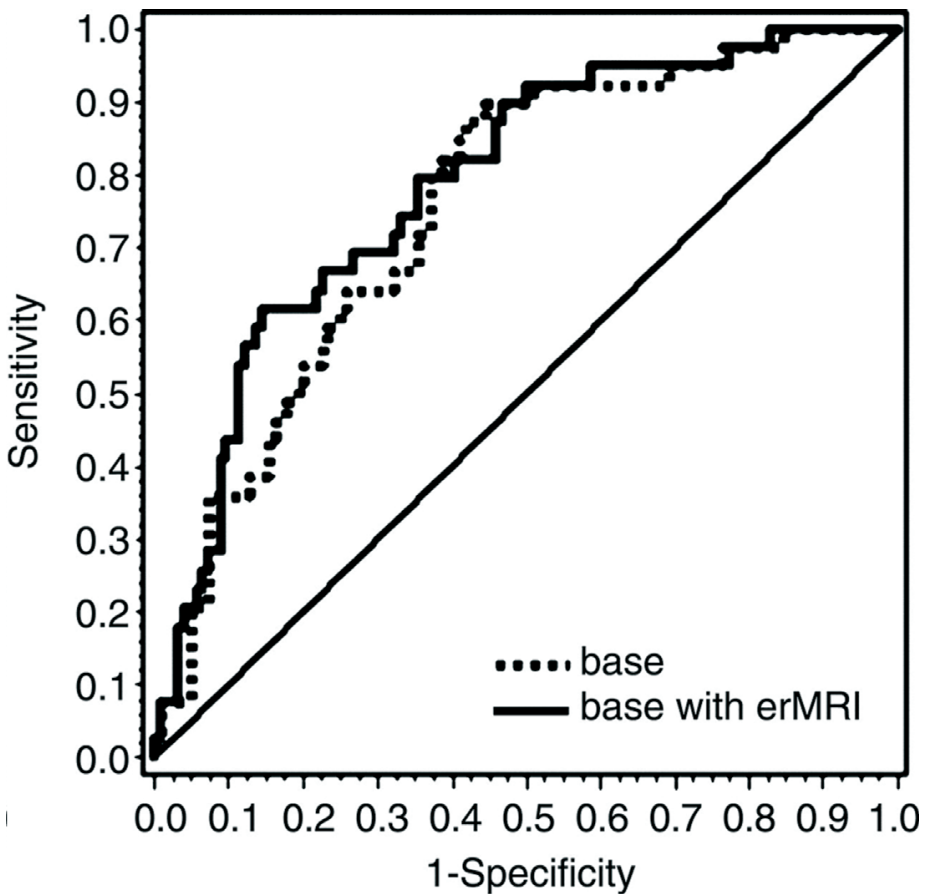

Figure 2a. Graphs show comparison of ROC curves for detection of ECE, with base model without and with endorectal MR imaging findings. Base model included the following variables: PSA level, Gleason score, greatest percentage of cancer in all core biopsy specimens, percentage of cancer-positive core specimens in all core biopsy specimens, presence of PNI, and clinical stage of tumor. Base model with endorectal MR imaging findings included MR imaging findings with all other variables. Predicted probability was calculated with jackknife method. Diagonal line indicates area under ROC curve of 0.500. (a) Graph shows ROC curves for genitourinary radiologist group. (b) Graph shows ROC curves for general body radiologist group.
Figure 2b. Graphs show comparison of ROC curves for detection of ECE, with base model without and with endorectal MR imaging findings. Base model included the following variables: PSA level, Gleason score, greatest percentage of cancer in all core biopsy specimens, percentage of cancer-positive core specimens in all core biopsy specimens, presence of $\mathrm{PNI}$, and clinical stage of tumor. Base model with endorectal MR imaging findings included MR imaging findings with all other variables. Predicted probability was calculated with jackknife method. Diagonal line indicates area under ROC curve of 0.500. (a) Graph shows ROC curves for genitourinary radiologist group. (b) Graph shows ROC curves for general body radiologist group.

In the general body radiologist group, in the model without MR imaging findings, PSA level $(P=.048)$ and greatest percentage of cancer in all core biopsy specimens $(P=.002)$ were predictors of ECE. When included in the model, endorectal MR imaging findings were significant predictors of ECE $(P=.002)$ (Table 6). The area under the ROC curve of the model with endorectal MR imaging findings, however, was not significantly greater than that of the model without endorectal MR imaging findings (area under the ROC curve $=0.813$ and 0.788 , respectively; $P=.31$ ) (Fig 2b). 


\section{Discussion}

The practice of medicine reflects a combination of knowledge and skills. As medicine advances, ever-narrowing subspecialties evolve. Patients benefit from the added clinical expertise (19-23). It has been demonstrated that the gain from treatment of a high volume of patients with a specific disease results in a better patient outcome (19).

Birkmeyer (20) estimated that for cardiovascular surgical procedures, such as coronary artery bypass grafting and abdominal aortic aneurysm repair, the mortality rates in patients treated by high-volume medical providers are 20\%-50\% lower than the mortality rates in those treated by low-volume providers. The association between patient volume and patient outcome does not stop at the cardiothoracic surgical ward. Researchers in studies (19) about the evaluation of cancer treatment demonstrated that patient volume can affect morbidity, local cancer recurrence, long-term survival, and mortality for a variety of cancer-related surgical procedures.

Investigators in several studies (21-23) have examined the relationship between patient volume and patient outcome with respect to the hospital or surgeon in patients who were undergoing radical prostatectomy. Findings in many of the studies indicated an inverse relationship between patient volume and mortality, morbidity, short- and long-term complications, and length of hospitalization (21-23). Not only surgeons succumb to the influences of experience and subspecialty training. When Gleason scores determined by pathologists were evaluated for accuracy, it was found that the pathologist's subspecialty influenced the disease grade. Gleason scores determined by general pathologists displayed only moderate interobserver reproducibility $(=0.435)$, whereas Gleason scores determined by genitourinary pathologists showed improved interobserver agreement $(=0.56-0.70)(24,25)$.

With consideration of these findings, it is not surprising that the accuracy of endorectal MR imaging findings and of MR image interpretation is related to radiologists' experience and subspecialty training (7,8,26-30). It has been demonstrated that endorectal MR imaging evaluations performed by experienced radiologists (those with more years of experience in interpretation of endorectal MR images of the prostate) have less interobserver variability and are more accurate than evaluations performed by inexperienced radiologists (31). Since the early reports about interobserver variability of MR imaging findings (6), both MR imaging technology and radiologists' skills have improved substantially. Yu et al (15) demonstrated that the combined use of endorectal MR imaging and MR spectroscopic imaging decreased interobserver variability and, for less experienced radiologists, significantly improved the detection of ECE in patients with prostate cancer.

Interobserver variability was not assessed in our study because interpretation of endorectal MR images was performed as part of the routine clinical service, and therefore only one radiologist interpreted the images for each case. For our data analysis, the ten MR imaging radiologists were classified as either genitourinary MR imaging radiologists or general body MR imaging radiologists. All of the readers were trained in body MR imaging (seven had an MR imaging fellowship and the others had been involved with MR imaging since it was introduced to clinical practice), but the genitourinary MR imaging radiologists had extensive experience in genitourinary imaging and more than 3 years of experience in prostate imaging. They regularly attended urology grand rounds and prostate tumor board conferences and were involved academically in the field.

Results of our study demonstrated that in the genitourinary radiologist group endorectal MR imaging findings displayed a combination of sensitivity and specificity that was superior to that of all other predictors tested. In the general body radiologist group, however, the combination of sensitivity and specificity of endorectal MR imaging findings was similar to that of the clinical predictors. Univariate analysis results revealed that most of the variables tested were significantly associated with findings of ECE. Results of multivariate analysis for assessment of two models of variables (one with and one without endorectal MR imaging findings) for their strength in the prediction of ECE demonstrated that in the genitourinary radiologist group a model with endorectal MR imaging findings had a greater area under the ROC curve than did a model without endorectal MR imaging findings (area under the ROC curve $=0.854$ and 0.760 , respectively; $P=.019$ ). In the general body radiologist group, however, the model with endorectal MR imaging findings did not have a significantly greater area under the ROC curve than did the model without endorectal MR imaging findings (area under the ROC curve $=0.813$ and 0.788 , respectively; $P=.31$ ). Nevertheless, endorectal MR imaging findings were significant predictors of ECE in both groups of patients. 
As stated by Kattan (32), predictors should not be judged by their $P$ value but rather by their ability to improve an existing set of clinically used variables. This argument applies to the evaluation of an expensive test such as endorectal MR imaging. Given the high incidence of prostate cancer, the cost of endorectal MR imaging might burden the health care system with additional expenses unless it results in fewer unnecessary surgeries or improves treatment planning and outcomes (33). D'Amico et al (34) concluded that although MR imaging findings added significant predictive value (ie, prediction of the risk of development of biochemical failure following radical prostatectomy in $20 \%$ of patients), the added predictive value of findings with this modality was not great enough to justify its routine use. Recently, it was suggested that treatment decisions should not be altered as a result of endorectal MR imaging findings (35).

One limitation of our study was that the contribution of MR spectroscopic imaging findings to the final MR imaging reports could not be assessed. MR spectroscopic technology was introduced at our institution in 2000, and radiologists experienced a steep learning curve in the acquisition and interpretation of MR spectroscopic imaging data during the past 4 years. Although MR spectroscopic imaging findings were accessible at the time of interpretation of endorectal MR imaging findings, readers did not always consult them, and therefore the degree of influence of MR spectroscopic imaging findings on reader impressions could not be quantified. Other limitations resulted from the fact that the study was designed to assess the value of endorectal MR image readings as used in clinical practice; accordingly, the readers were not blinded to clinical data such as PSA level and biopsy results, and as mentioned before, only one reader evaluated images in each case once so that we were unable to assess interobserver and intraobserver variability.

With consideration of the foregoing data, results of this study demonstrate that endorectal MR imaging findings add significant value in the diagnosis of ECE in patients with prostate cancer when MR images are interpreted by radiologists with experience in MR imaging of the prostate. In light of these results, it is easy to understand why referring physicians have experienced frustration about the use of MR image readings for the evaluation of prostate cancer. Nevertheless, advances in technology and in the expertise of radiologists dedicated to the genitourinary field suggest that endorectal MR imaging could play an increasingly useful role in the treatment of patients with prostate cancer.

In conclusion, results of this study demonstrate that endorectal MR imaging findings can be significant predictors for ECE in patients with prostate cancer, after controlling for PSA level, Gleason score, greatest percentage of cancer in all core biopsy specimens, percentage of cancer-positive core specimens in all core biopsy specimens, PNI, and clinical stage of tumor. A comparison of the ROC curves drawn from the results of readings performed by genitourinary MR imaging radiologists and general body MR imaging radiologists showed that endorectal MR imaging findings added value to all other predictor variables only when MR images were interpreted by genitourinary MR imaging radiologists.

\section{References}

1. Partin AW, Mangold LA, Lamm DM, Walsh PC, Epstein Jl, Pearson JD. Contemporary update of prostate cancer staging nomograms (Partin Tables) for the new millennium. Urology 2001; 58:843-848.

2. Kattan MW, Stapleton AM, Wheeler TM, Scardino PT. Evaluation of a nomogram used to predict the pathologic stage of clinically localized prostate carcinoma. Cancer 1997; 79:528-537.

3. Graefen M, Karakiewicz Pl, Cagiannos I, et al. Validation study of the accuracy of a postoperative nomogram for recurrence after radical prostatectomy for localized prostate cancer. J Clin Oncol 2002; 20:951-956.

4. McSherry S, Levy F, Schiebler ML, Keefe B, Dent GA, Mohler JL. Preoperative prediction of pathological tumor volume and stage in clinically localized prostate cancer: comparison of digital rectal examination, transrectal ultrasonography and magnetic resonance imaging. J Urol 1991; 146:85-89.

5. Schiebler M, McSherry S, Keefe B, et al. Comparison of the digital rectal examination, endorectal ultrasound, and body coil magnetic resonance imaging in the staging of adenocarcinoma of the prostate. Urol Radiol 1991; 13:110-118.

6. Rifkin MD, Zerhouni EA, Gatsonis CA, et al. Comparison of magnetic resonance imaging and ultrasonography in staging early prostate cancer: results of a multi-institutional cooperative trial. N Engl J Med 1990; 323:621-626.

7. Schiebler ML, Yankaskas BC, Tempany C, et al. MR imaging in adenocarcinoma of the prostate: interobserver variation and efficacy for determining stage C disease. AJR Am J Roentgenol 1992; 158:559-562; discussion 563-564.

8. Tempany CM, Zhou X, Zerhouni EA, et al. Staging of prostate cancer: results of Radiology Diagnostic Oncology Group project comparison of three MR imaging techniques. Radiology 1994; 192:47-54

9. Walsh PC. Editorial comment (letter). J Urol 2000; 163:1375.

10. Wang L, Mullerad M, Chen HN, et al. Prostate cancer: incremental value of endorectal MR imaging findings for prediction of extracapsular extension. Radiology 2004; 232:133-139.

11. Bottomley PA. Selective volume method for performing localized MR spectroscopy. U.S. patent 44802281984. 
12. Star-Lack J, Nelson SJ, Kurhanewicz J, Huang LR, Vigneron DB. Improved water and lipid suppression for 3D PRESS CSI using RF band 'selective inversion with gradient dephasing (BASING). Magn Reson Med 1997; 38:311-321.

13. Brown TR, Kincaid BM, Ugurbil K. NMR chemical shift imaging in three dimensions. Proc Natl Acad Sci U S A 1982; 79:3523-3526.

14. Nelson S, Day MR, Carvajal L, et al. Methods for analysis of serial volume MRI and $1 \mathrm{H}$ MRS data for the assessment of response to therapy in patients with brain tumors (abstr) In: Proceedings of the Society of Magnetic Resonance in Medicine and the European Society of Magnetic Resonance in Medicine and Biology. Berkeley, Calif: Society of Magnetic Resonance in Medicine, 1995.

15. Yu KK, Scheidler J, Hricak H, et al. Prostate cancer: prediction of extracapsular extension with endorectal MR imaging and threedimensional proton MR spectroscopic imaging. Radiology 1999; 213:481-488.

16. Rubin MA, Bassily N, Sanda M, Montie J, Strawderman MS, Wojno K. Relationship and significance of greatest percentage of tumor and perineural invasion on needle biopsy in prostatic adenocarcinoma. Am J Surg Pathol 2000; 24:183-189.

17. Bismar TA, Lewis JS, Jr, Vollmer RT, Humphrey PA. Multiple measures of carcinoma extent versus perineural invasion in prostate needle biopsy tissue in prediction of pathologic stage in a screening population. Am J Surg Pathol 2003; 27:432-440.

18. Yossepowitch $\mathrm{O}$, Sircar K, Scardino PT, et al. Bladder neck involvement in pathological stage pT4 radical prostatectomy specimens is not an independent prognostic factor. J Urol 2002; 168:2011-2015.

19. Shahian DM, Normand SL. The volume-outcome relationship: from Luft to Leapfrog. Ann Thorac Surg 2003; 75:1048-1058.

20. Birkmeyer JD. Should we regionalize major surgery? potential benefits and policy considerations. J Am Coll Surg 2000; 190:341-349.

21. Yao SL, Lu-Yao G. Population-based study of relationships between hospital volume of prostatectomies, patient outcomes, and length of hospital stay. J Natl Cancer Inst 1999; 91:1950-1956.

22. Ellison LM, Heaney JA, Birkmeyer JD. The effect of hospital volume on mortality and resource use after radical prostatectomy. J Urol 2000; 163:867-869.

23. Begg CB, Riedel ER, Bach PB, et al. Variations in morbidity after radical prostatectomy. N Engl J Med 2002; 346:1138-1144.

24. Allsbrook WC, Jr, Mangold KA, Johnson MH, Lane RB, Lane CG, Epstein JI. Interobserver reproducibility of Gleason grading of prostatic carcinoma: general pathologist. Hum Pathol 2001; 32:81-88.

25. Allsbrook WC, Jr, Mangold KA, Johnson MH, et al. Interobserver reproducibility of Gleason grading of prostatic carcinoma: urologic pathologists. Hum Pathol 2001; 32:74-80.

26. Dudley RA, Hricak H, Scheidler J, et al. Shared patient analysis: a method to assess the clinical benefits of patient referrals. Med Care 2001; 39:1182-1187.

27. Langlotz CP. Benefits and costs of MR imaging of prostate cancer. Magn Reson Imaging Clin N Am 1996; 4:533-544.

28. Berlin L. Reporting the "missed" radiologic diagnosis: medicolegal and ethical considerations. Radiology 1994; 192:183-187.

29. Kalbhen CL, Yetter EM, Olson MC, Posniak HV, Aranha GV. Assessing the resectability of pancreatic carcinoma: the value of reinterpreting abdominal CT performed at other institutions. AJR Am J Roentgenol 1998; 171:1571-1576.

30. Sickles EA, Wolverton DE, Dee KE. Performance parameters for screening and diagnostic mammography: specialist and general radiologists. Radiology 2002; 224:861-869.

31. Tsuda K, Yu KK, Coakley FV, Srivastav SK, Scheidler JE, Hricak H. Detection of extracapsular extension of prostate cancer: role of fat suppression endorectal MRI. J Comput Assist Tomogr 1999; 23:74-78.

32. Kattan MW. Judging new markers by their ability to improve predictive accuracy. J Natl Cancer Inst 2003; 95:634-635.

33. Penson DF, Litwin MS, Aaronson NK. Health related quality of life in men with prostate cancer. J Urol 2003; 169:1653-1661.

34. D'Amico AV, Whittington R, Malkowicz B, et al. Endorectal magnetic resonance imaging as a predictor of biochemical outcome after radical prostatectomy in men with clinically localized prostate cancer. J Urol 2000; 164:759-763.

35. May F, Treumann T, Dettmar P, Hartung R, Breul J. Limited value of endorectal magnetic resonance imaging and transrectal ultrasonography in the staging of clinically localized prostate cancer. BJU Int 2001; 87:66-69. 
4 
Prediction of Seminal Vesicle Invasion in Prostate Cancer: Incremental Value of Adding Endorectal MR Imaging to the Kattan Nomogram 


\section{Prediction of Seminal Vesicle Invasion in Prostate Cancer: Incremental Value of Adding Endorectal MR Imaging to the Kattan Nomogram}

Supported by National Institutes of Health grant R01 CA76423.

Liang Wang, Hedvig Hricak, Michael W. Kattan, Hui Ni Chen, Kentaro Kuroiwa, Halley F. Eisenberg and Peter T. Scardino

From the Departments of Radiology (L.W., H.H., H.N.C., H.F.E.) and Urology (K.K., P.T.S.), Memorial Sloan-Kettering Cancer Center, 1275 York Ave, New York, NY 10021; and Department of Quantitative Health Sciences, Cleveland Clinic Foundation, Cleveland, Ohio (M.W.K.). Received July 26, 2005; revision requested September 13; revision received January 23, 2006; accepted February 13; final version accepted May 19.

\section{Abstract}

Purpose: To retrospectively determine whether endorectal magnetic resonance (MR) imaging findings contribute incremental value to the Kattan nomogram for predicting seminal vesicle invasion (SVI) in patients with prostate cancer.

Materials and Methods: The institutional review board issued a waiver of authorization, which included a waiver of informed consent, for this HIPAA-compliant study. From October 2000 through January 2005, 573 patients (mean age, 58.3 years; age range, 36-86 years) underwent endorectal MR imaging before prostate cancer surgery. The endorectal MR imaging results had been prospectively interpreted by seven radiologists, and the likelihood of SVI was retrospectively scored on the basis of radiologists' written reports. MR imaging findings, individual clinical variables (serum prostate-specific antigen [PSA] level, Gleason grade, clinical stage, greatest percentage of cancer in all biopsy cores, percentage of positive cores in all biopsy cores, and perineural invasion), and the Kattan nomogram were evaluated with respect to SVI prediction; surgical pathologic analysis was used as the reference standard. Logistic regression and receiver operating characteristic $(\mathrm{ROC})$ curve analyses were performed.

Results: At pathologic analysis, 28 (4.9\%) of 573 patients had SVI. At univariate analysis, endorectal MR imaging results and all clinical variables except the percentage of positive biopsy cores were significantly associated with SVI ( $<.02)$; endorectal MR imaging (0.76) had a larger area under the ROC curve (AUC) than any clinical variable (0.62-0.73). At multivariate analysis, endorectal MR imaging results, Gleason grade, PSA level, and the percentage of cancer in all biopsy cores were significantly associated with SVI $(P \leq .02)$. The Kattan nomogram plus endorectal MR imaging (0.87) had a significantly larger $(P<.05)$ AUC than either endorectal MR imaging alone $(0.76)$ or the Kattan nomogram alone (0.80).

Conclusion: The addition of endorectal MR imaging contributes significant incremental value to the Kattan nomogram for predicting SVI.

(c) RSNA, 2006

\section{AdVANCES IN KNOWLEDge}

- At univariate and multivariate analyses, endorectal MR imaging findings are a significant presurgical predictor of seminal vesicle invasion $(P<.001)$.

- Endorectal MR imaging findings contribute significant incremental value to the Kattan nomogram for predicting seminal vesicle invasion $(P=.04)$. 


\section{INTRODUCTION}

$\mathbf{P}$

rostate cancer has the highest incidence of any noncutaneous cancer in the United States and is the third leading cause of cancer death in American men (1). The American Cancer Society has estimated that in 2006 in the United States, 234460 new cases of prostate cancer will be diagnosed and 27350 men will die of the disease (1). The prevalence of seminal vesicle invasion (SVI) ${ }^{1}$ in contemporary surgical series (ie, those series performed in the past 10 years) is 5\%-23\% (2-16). Preoperative identification of SVI is an important factor for staging and prognosis and may modify treatment selection and treatment planning $(4,12,17)$.

The prognosis, however, depends on the status of other tumor prognostic variables and is not always poor. In a recent study (4), the 5-year biochemical progression-free rate for patients with SVI was $71.9 \%$ when surgical margins and nodes were negative, $36.6 \%$ when surgical margins were positive and nodes were negative, and $25.9 \%$ when surgical margins and nodes were positive.

Clinical factors associated with an increased incidence of SVI include a high prostate-specific antigen (PSA) level, a high Gleason grade, the presence of tumor at the base of the prostate gland, and lymph node metastasis $(3,4,18,19)$. To replace somewhat arbitrary combinations of individual clinical variables, nomograms have been introduced to help predict the stage of prostate cancer and to aid in the choice of treatment (3,20-22). The Kattan staging nomogram, which is based on presurgical clinical variables (serum PSA level, Gleason grade at biopsy, clinical stage, and systematic needle biopsy cores from the base of the prostate), is a validated predictive instrument that is widely used to help direct adjuvant therapy and to guide postoperative counseling for patients with clinical data suggestive of SVI $(3,23)$. However, the Kattan staging nomogram cannot predict the location of SVI. Of the modern diagnostic imaging modalities, endorectal magnetic resonance (MR) imaging has shown the most promise in the detection of prostate cancer SVI, with high specificity $(81 \%-99 \%)$ but widely varying sensitivity $(23 \%-80 \%)(6-9,14-16,24)$.

In historical studies on the detection of SVI with MR imaging, the disease was often relatively advanced, and the contribution of MR findings to clinical nomograms was not assessed $(5-9,14-16,24)$. Thus, the purpose of our study was to retrospectively determine whether endorectal MR imaging findings contribute incremental value to the Kattan nomogram for predicting SVI in patients with prostate cancer.

\section{Materials and Methods}

\section{Patients}

A total of 592 consecutive patients with prostate cancer were referred from the urology department to undergo MR imaging before radical retropubic prostatectomy and pelvic lymphadenectomy, which were performed at our institution by one of six attending surgeons, one of whom was an author (P.T.S.) (Table 1). For the six attending surgeons, the range of experience in prostate surgery was 10-35 years. From October 2000 through June 2003, imaging was performed as part of an ongoing National Institutes of Health study investigating the use of MR imaging in patients with prostate cancer; all patients gave informed consent before enrollment in the prospective institutional review board-approved National Institutes of Health study, which was compliant with the Health Insurance Portability and Accountability Act. From July 2003 to January 2005, imaging was performed as part of our accepted clinical practice for patient evaluation. The institutional review board of the Memorial Sloan-Kettering Cancer Center issued a waiver of authorization (which includes a waiver of informed consent) for retrospective review of the MR imaging reports and clinical data; this review was also compliant with the Health Insurance Portability and Accountability Act.

\footnotetext{
${ }^{1}$ Abbreviations

$\mathrm{AUC}=$ area under the $\mathrm{ROC}$ curve $\cdot \mathrm{PNI}=$ perineural invasion $\cdot \mathrm{PSA}=$ prostate-specific antigen $\cdot \mathrm{ROC}=$ receiver operating characteristic $\cdot \mathrm{SVI}=$ seminal vesicle invasion
} 


\begin{tabular}{|c|c|}
\hline Clinical Variable & Value \\
\hline PSA level $(\mathrm{ng} / \mathrm{mL})^{*}$ & $5.4 \pm 4.28(0-50.00)$ \\
\hline \multicolumn{2}{|l|}{ Gleason grade at biopsy } \\
\hline $3+2$ & $2(0.3)$ \\
\hline $3+3$ & $364(63.5)$ \\
\hline $3+4$ & $126(22.0)$ \\
\hline $4+3$ & $53(9.2)$ \\
\hline $4+4$ or $3+5$ & $22(3.8)$ \\
\hline $4+5$ & $5(0.9)$ \\
\hline $5+4$ & $1(0.2)$ \\
\hline Greatest percentage of cancer in all biopsy cores* & $0.20 \pm 0.23(0-1.00)$ \\
\hline Percentage of positive cores in all biopsy cores* & $0.36 \pm 0.26(0-1.00)$ \\
\hline \multicolumn{2}{|l|}{ PNI at biopsy } \\
\hline Absent & $500(87.3)$ \\
\hline Present & $73(12.7)$ \\
\hline \multicolumn{2}{|l|}{ Clinical stage at biopsy } \\
\hline $\mathrm{T} 1 \mathrm{~b}$ & $2(0.3)$ \\
\hline T1c & $361(63.0)$ \\
\hline $\mathrm{T} 2 \mathrm{a}$ & $121(63.0)$ \\
\hline $\mathrm{T} 2 \mathrm{~b}$ & $50(8.7)$ \\
\hline $\mathrm{T} 2 \mathrm{C}$ & $23(4.0)$ \\
\hline T3 & $16(2.8)$ \\
\hline
\end{tabular}

Note.-Unless otherwise indicated, data are the number of patients; data in parentheses are percentages.

* Data are the median \pm standard deviation; data in parentheses are ranges.

For 19 of 592 patients, no needle biopsy cores from the base of the prostate were available, and thus the likelihood of SVI on the basis of the Kattan nomogram could not be calculated (3). Therefore, data analysis was based on the remaining 573 patients (mean age, 58.3 years; age range, 36-86 years). None of the 573 patients underwent neoadjuvant hormonal therapy or radiation therapy prior to surgery. The average time between biopsy and MR imaging was 71.73 days (range, 1042 days before MR imaging to 114 days after MR imaging), and the average time from MR imaging to surgery was 38.40 days (range, 1-185 days).

In all patients, prostate cancer was diagnosed by means of biopsy specimens. Clinical serum PSA levels, Gleason grade at biopsy, clinical stage, the greatest percentage of cancer in all biopsy cores, the percentage of positive cores in all biopsy cores, and perineural invasion (PNI) were recorded from the patients' medical records (K.K.). A portion of the patient population has been reported on previously (25-28).

\section{Kattan Staging Nomogram}

For all 573 patients, the likelihood of SVI according to the Kattan nomogram was recorded by two authors (L.W. and K.K. working together) on the basis of serum PSA levels, Gleason grade at biopsy, clinical stage, and systematic needle biopsy cores from the base of the prostate (3).

\section{MR Data Acquisition and Interpretation}

MR imaging was performed with a 1.5-T whole-body MR imager (Signa Horizon; GE Medical Systems, Milwaukee, Wis). Patients were examined in the supine position by using the body coil for excitation and a pelvic phased-array 
coil (GE Medical Systems) in combination with a commercially available balloon-covered expandable endorectal coil (Medrad, Pittsburgh, Pa) for signal reception. T1-weighted transverse and spin-echo MR images were obtained from the aortic bifurcation to the symphysis pubis by using the following parameters: repetition time, 700 msec; echo time, 8 msec; section thickness, 5 mm; intersection gap, $1 \mathrm{~mm}$; field of view, $24 \mathrm{~cm}$; matrix, 256 × 192; transverse frequency direction (to prevent obstruction of the pelvic node by endorectal coil motion artifact); and one signal acquired. Thin-section high-spatial-resolution transverse and coronal T2-weighted fast spin-echo MR images of the prostate and seminal vesicles were obtained by using the following parameters: repetition time, $5000 \mathrm{msec}$; effective echo time, 96 msec; echo train length, 16; section thickness, 3 mm; no intersection gap; field of view, 14 cm; matrix, $256 \times 192$; anteroposterior frequency direction (to prevent obstruction of the prostate by endorectal coil motion artifact); and four signals acquired.

MR images were prospectively interpreted by seven attending MR body radiologists, one of whom was an author $(\mathrm{H} . \mathrm{H}$.); images were interpreted independently during the regular clinical assignment of each radiologist to the MR

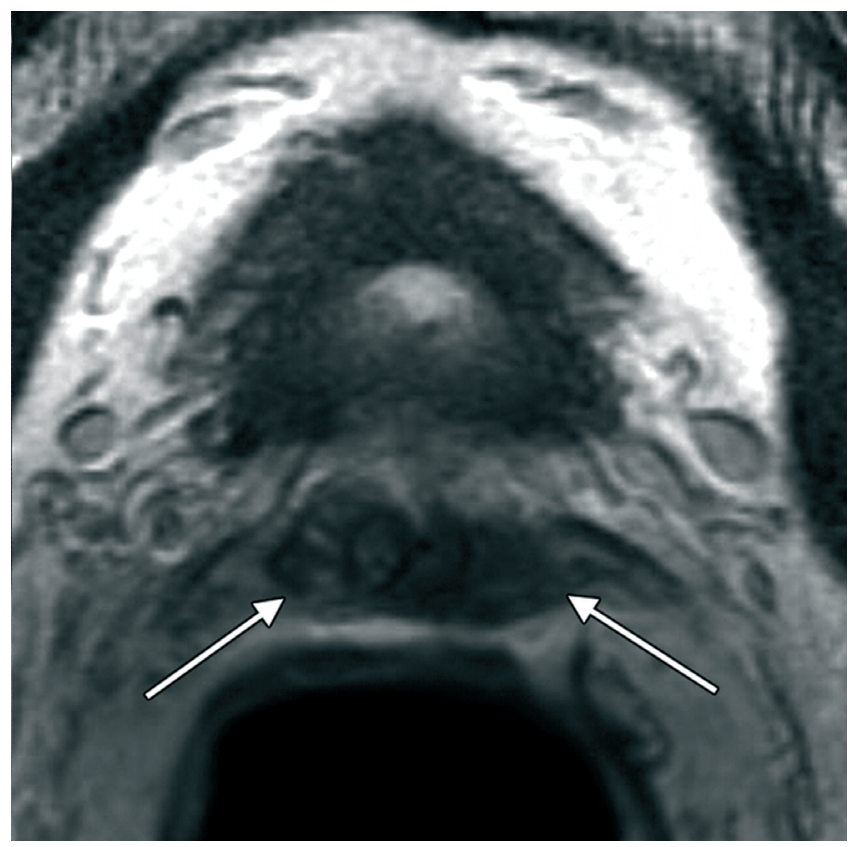

Figure 1a: pT4 prostate cancer (clinical stage T2b with PSA level of $12.42 \mathrm{ng} / \mathrm{mL}$ ) and bilateral seminal vesicle involvement in a 63-year-old man. Sextant biopsy results showed Gleason grade $4+3$ tumors involving 50\% of the total submitted tissue on both sides ( $94 \%$ of total submitted cores were positive) and PNI in left peripheral and transition zones. According to the Kattan staging nomogram, the likelihood of SVI was 18\%. (a) Transverse T2weighted fast spin-echo 3-mm-thick MR image (4900/118 [repetition time msec/effective echo time $\mathrm{msec}]$ ) and (b) coronal T2-weighted fast spin-echo 3-mm-thick MR image (4666/96) show bilateral SVI (arrows). (c) Wholemount pathologic step section shows prostate cancer with bilateral seminal vesicle involvement. The green circle represents the area with a Gleason grade of 3, and the black circle represents the area with a Gleason grade of 4 or a mixture of Gleason grades 4 and 5. LSV = left seminal vesicle, RSV = right seminal vesicle.

service. Six of the readers had performed a fellowship in body imaging that included MR imaging. The experience of each radiologist in reading MR images since fellowship ranged from 1 to 13 years, with one reader having more than 20 years of experience interpreting MR images. All of the readers had experience in prostate imaging and were members of the institution's prostate cancer disease management team. As per regular clinical practice, the readers were not blinded to clinical data, such as PSA level and biopsy results; however, the amount of clinical data that was available varied. Each radiologist made his or her determination regarding the presence of SVI on the basis of his or her own continuous medical training and knowledge of previously described MR features of SVI.

The main diagnostic criteria used by the radiologists to determine the presence of SVI on MR images were the disruption or loss of the normal architecture of the seminal vesicle, focal or diffuse areas of low signal intensity within the seminal vesicle, low signal intensity within the seminal vesicle causing mass effect, enlarged ejaculatory ducts with low signal intensity, and direct extension of the low signal intensity of tumor from the base of the prostate to the seminal vesicle (24). On the basis of the radiologists' written reports, one author (L.W.) independently and retrospectively scored the likelihood of SVI on a scale of $1-5$, with a score of 1 indicating no SVI; 2 , probably no SVI (ie, SVI cannot be ruled out, although there is no clear evidence); 3 , possible SVI (lesion is suspicious for SVI); 4, probable SVI (lesion is highly suspicious for SVI); and 5, definite SVI. 

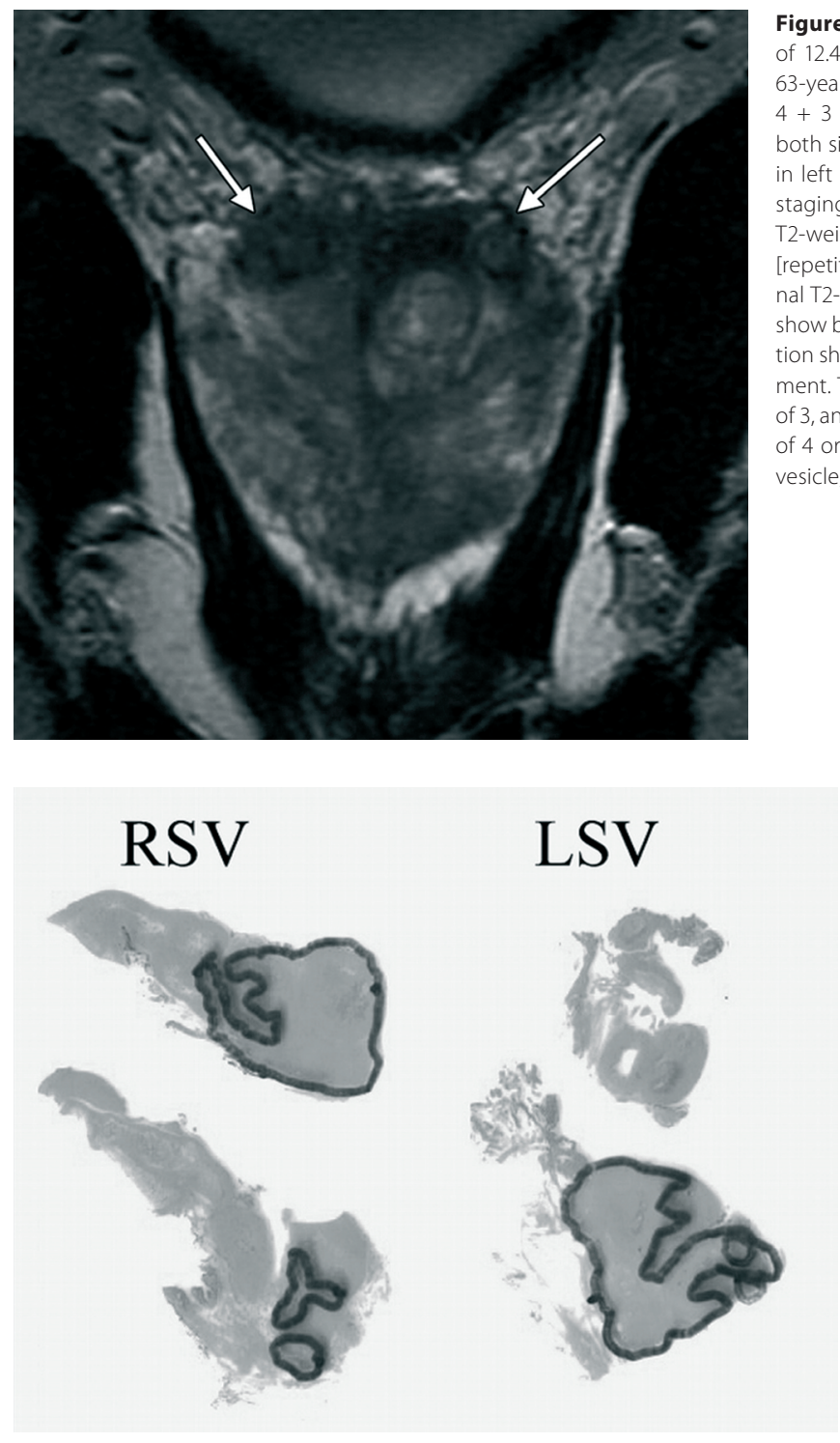

Figure 1b: pT4 prostate cancer (clinical stage T2b with PSA level of $12.42 \mathrm{ng} / \mathrm{mL}$ ) and bilateral seminal vesicle involvement in a 63-year-old man. Sextant biopsy results showed Gleason grade $4+3$ tumors involving $50 \%$ of the total submitted tissue on both sides ( $94 \%$ of total submitted cores were positive) and PNI in left peripheral and transition zones. According to the Kattan staging nomogram, the likelihood of SVI was 18\%. (a) Transverse T2-weighted fast spin-echo 3-mm-thick MR image (4900/118 [repetition time msec/effective echo time msec]) and (b) coronal T2-weighted fast spin-echo 3-mm-thick MR image (4666/96) show bilateral SVI (arrows). (c) Whole-mount pathologic step section shows prostate cancer with bilateral seminal vesicle involvement. The green circle represents the area with a Gleason grade of 3, and the black circle represents the area with a Gleason grade of 4 or a mixture of Gleason grades 4 and 5 . LSV = left seminal vesicle, RSV = right seminal vesicle.

\section{Histopathologic Analysis}

Figure 1c: pT4 prostate cancer (clinical stage T2b with PSA level of $12.42 \mathrm{ng} / \mathrm{mL}$ ) and bilateral seminal vesicle involvement in a 63-year-old man. Sextant biopsy results showed Gleason grade $4+3$ tumors involving $50 \%$ of the total submitted tissue on both sides ( $94 \%$ of total submitted cores were positive) and PNI in left peripheral and transition zones. According to the Kattan staging nomogram, the likelihood of SVI was $18 \%$. (a) Transverse T2-weighted fast spin-echo 3-mm-thick MR image (4900/118 [repetition time msec/effective echo time $\mathrm{msec}]$ ) and (b) coronal T2-weighted fast spinecho 3-mm-thick MR image (4666/96) show bilateral SVI (arrows). (c) Whole-mount pathologic step section shows prostate cancer with bilateral seminal vesicle involvement. The green circle represents the area with a Gleason grade of 3, and the black circle represents the area with a Gleason grade of 4 or a mixture of Gleason grades 4 and 5 . LSV = left seminal vesicle, RSV = right seminal vesicle.

(Color version available in chapter 18).

Pretreatment sextant biopsy reports provided the following clinical parameters, which were used for prediction of SVI: Gleason grade, the greatest percentage of cancer in all biopsy cores, the percentage of positive cores in all biopsy cores, and PNI. Radical prostatectomy specimens had been examined by the institutional pathology department. Prostatectomy specimens were inked with tattoo dye (green dye on the right side and blue dye on the left side), and the specimens were fixed in 10\% formalin for 36 hours. The distal $5 \mathrm{~mm}$ of the apex was amputated and coned. The remainder of the gland was serially sectioned from the apex to the base at 3-4-mm intervals and was entirely submitted for paraffin embedding as whole-mount sections. The seminal vesicles were amputated and submitted separately. After paraffin embedding, microsections were placed on glass slides and were stained with hematoxylin and eosin. One uropathologist (K.K.) subsequently reviewed all the specimens, mapped each tumor area with a marker (green for a Gleason grade of 3 and black for a Gleason grade of 4 or a mixture of Gleason grades 4 and 5), and recorded the presence and location of SVI (Fig 1). 


\section{Statistical Analysis}

Univariate and multivariate logistic regression and receiver operating characteristic (ROC) curve analyses were performed for all clinical and imaging variables to predict SVI (H.N.C., M.W.K.). Univariate logistic regression was used to compare the characteristics of patients with SVI with the characteristics of patients without SVI. The area under the ROC curve (AUC) was evaluated for models of SVI prediction on the basis of the Kattan nomogram alone and MR imaging alone. In addition, a model was constructed for the prediction of SVI on the basis of the Kattan nomogram and MR imaging combined. AUC was bias-corrected by using the leave-one-out cross validation method (29). A P value of less than .05 was considered to indicate a statistically significant difference. The software programs that were used for data analysis included SAS (version 8.2; SAS Institute, Cary, NC) and S-PLUS (version 2000; Insightful, Seattle, Wash).

\section{ResUlts}

At surgical histopathologic analysis, 28 (4.9\%) of 573 patients had evidence of SVI. Unilateral SVI was present in 22 (3.8\%) of 573 patients, and bilateral SVI was present in six (1.0\%) of 573 patients (Table 2).

MR imaging was associated with SVI at both univariate and multivariate analysis ( $P<.001$ in both cases) (Table 3).

\section{Table 2. Prevalence of SVI in Final Pathologic Specimens}

\begin{tabular}{lc} 
SVI & $\begin{array}{c}\text { No. of Patients } \\
(\boldsymbol{n}=\mathbf{5 7 3})\end{array}$ \\
\hline Absent & $545(95.1)$ \\
Present & $28(4.9)$ \\
Unilateral & $22(3.8)$ \\
Bilateral & $6(1.0)$
\end{tabular}

Note.-Data in parentheses are percentages.

Table 3. Strength of Clinical Variables and MR Findings for the Prediction of SVI

\begin{tabular}{|lcc|}
\hline Clinical Variables and MR Findings & Univariate Analysis* & Multivariate Analysis* \\
\hline MR findings & $<.001(0.76)$ & $<.001$ \\
\hline $\begin{array}{l}\text { Greatest percentage of cancer in all } \\
\text { biopsy cores }\end{array}$ & $<.001(0.73)$ & .02 \\
\hline PSA level & $.014(0.72)$ & .012 \\
\hline Clinical stage & $<.001(0.71)$ & .23 \\
\hline Gleason grade & $.01(0.70)$ & $<.001$ \\
\hline PNI & $.005(0.62)$ & .55 \\
\hline $\begin{array}{l}\text { Percentage of positive cores in all biopsy } \\
\text { cores }\end{array}$ & $.30(0.67)$ & .08 \\
\hline
\end{tabular}

Note.-Data are P values. Predictor variables were treated as continuous except for Gleason grade (categorized as $3+3,3+4 \mathrm{vs} 3+5,4+3,4+4$, $4+5$, and $5+5$ ) and clinical stage (categorized as T1 vs T2).

* Data in parentheses are AUC values.

At univariate analysis, MR findings ( $P<.001)$, PSA level $(P=.014)$, Gleason grade $(P=.01)$, clinical stage $(P<.001)$, the greatest percentage of cancer in all biopsy cores $(P<.001)$, and PNI $(P=.005)$ were significantly associated with SVI; the percentage of positive cores was the only clinical variable that was not significantly associated with $\mathrm{SVI}(\mathrm{P}=.30)$. AUC was larger for MR imaging (0.76) than for any of the clinical variables (0.62-0.73) (Table 3). 
At multivariate analysis, MR findings ( $P<.001)$, PSA level $(P=.012)$, Gleason grade $(P<.001)$, and the greatest percentage of cancer in all biopsy cores $(P=.02)$ were significantly associated with SVI. The AUC for the Kattan nomogram plus MR imaging (0.87) was significantly larger than the AUC for the Kattan nomogram alone (0.80) or for MR imaging alone $(0.76)(P<.05$ in both cases) (Table 4, Figs 1, 2).

Table 4. AUC Values for Models Predicting SVI on the Basis of MR Imaging and the Kattan Nomogram

\begin{tabular}{lcc} 
Model & AUC Value & P Value* \\
\hline Kattan nomogram & 0.80 & .04 \\
MR imaging & 0.76 & .01 \\
MR imaging plus Kattan nomogram & 0.87 &
\end{tabular}

Note.-The model containing the Kattan nomogram plus MR imaging had a significantly greater AUC value (0.87) than either the Kattan nomogram alone $(0.80)$ or MR imaging alone $(0.76)(P<.05$ in both cases).

*P values were calculated by comparing each model with MR imaging plus the Kattan nomogram.

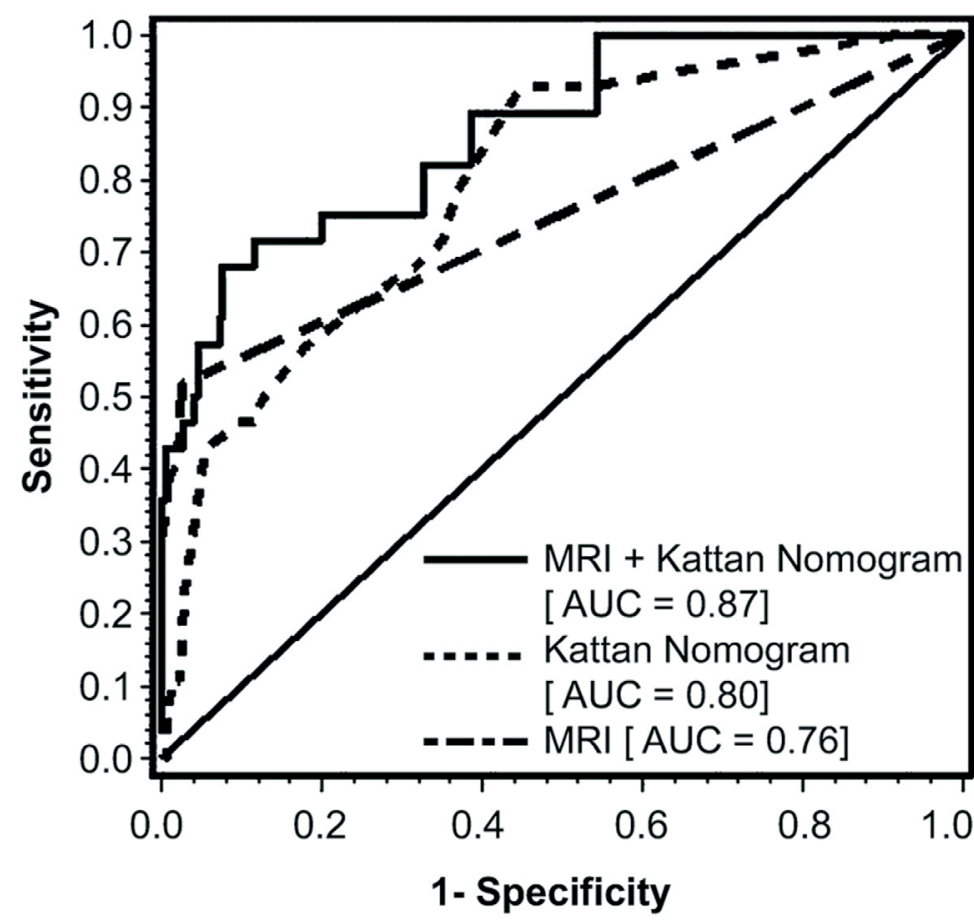

Figure 2: Graph shows ROC curves for jackknife predicted probabilities of SVI on the basis of endorectal MR imaging and the Kattan nomogram plus MR imaging. The model containing the Kattan nomogram plus MR imaging (0.87) has a significantly greater AUC than the model containing only the Kattan nomogram $(0.80 ; \mathrm{P}=.04)$ or the model containing only MR imaging $(0.76 ; \mathrm{P}<.01)$.

\section{Discussion}

In patients with prostate cancer, the presence of SVI is associated with an increased risk of lymph node metastasis and recurrence $(4,17,23,30)$. The prediction of SVI before treatment may influence treatment selection in favor of radiation therapy instead of surgery. Furthermore, while resection of the seminal vesicles has been a standard component of radical prostatectomy, it has recently been suggested that if SVI can be confidently ruled out, the surgeon may prefer to spare the seminal vesicles during radical prostatectomy to prevent long-term loss of urinary continence (31). 
In our patient population, $4.9 \%$ of patients had evidence of SVI at surgical histopathologic analysis. This result is consistent with the dramatic downstaging of prostate cancer at the time of diagnosis that has occurred over the past 2 decades, as demonstrated by the National Cancer Institute's Surveillance, Epidemiology, and End Results Program. Data from this program show that from 1995 to 2001, 91\% of prostate cancer cases were of a local or regional stage at diagnosis compared with only 74\% from 1981 to $1987(1,32)$.

Because of the importance of SVI in patient prognosis and treatment choice, presurgical biopsy of the seminal vesicles has been suggested. Terris et al (33) performed biopsies of the seminal vesicles in 73 selected patients, 12 of whom had SVI in the radical prostatectomy specimens. They reported a negative predictive value of $97 \%$, a positive predictive value of $80 \%$, and a sensitivity of $67 \%$. The low incidence of SVI overall, occasional false-positive results, and low sensitivity limit the enthusiasm for performing biopsy in the seminal vesicles $(3,12,33)$.

In the literature, serum PSA level and Gleason grade are significantly associated with SVI for both univariate and multivariate analysis (3,34-36). In our study, MR imaging results, the greatest percentage of cancer in all biopsy cores, PSA level, clinical stage, Gleason grade, and PNI were all significantly associated with SVI at univariate analysis, while MR imaging results, Gleason grade, PSA level, and the greatest percentage of cancer in all biopsy cores were significantly associated with SVI at multivariate analysis. Koh et al (3) found that all prognostic factors, when combined in a model, predicted the pathologic stage more accurately than did any single parameter alone — a finding that is consistent with the findings of our study. Our results show that MR imaging can add significant incremental value to clinical variables for the prediction of SVI.

The role of modern MR imaging in prostate cancer management has been changing not only because of the development of new MR techniques, such as endorectal MR imaging, contrast material-enhanced endorectal MR imaging, and MR spectroscopic imaging, but also because of radiologists' increased experience with MR imaging of the prostate. The results of previous studies have shown that MR imaging findings, although subject to substantial interobserver variability, can add significant incremental value to clinical variables in predicting other features of prostate cancer that are relevant to staging, such as extracapsular extension and lymph node metastasis $(26,27,37)$.

Our study was not designed to assess MR technology but to assess the value of MR imaging, as applied in the clinical setting during radiologists' regular clinical assignments. Accordingly, the readers had access to clinical data, such as PSA level and biopsy results when available, in the patient's medical record. Each study was evaluated only once, so we could not assess interobserver and intraobserver variability. Furthermore, we studied a consecutive cohort of patients who met eligibility requirements on the basis of pretreatment factors (not SVI outcome) and not a random sample. Thus, the estimates of accuracy might be biased.

In conclusion, our results show that endorectal MR imaging findings can contribute significant incremental value to the Kattan nomogram for predicting SVI. Although further multicenter confirmatory studies would be helpful, we suggest that MR imaging has an important role to play in the pretreatment evaluation of prostate cancer, including the prediction of SVI.

\section{References}

1. Jemal A, Siegel R, Ward E, et al. Cancer statistics, 2006. CA Cancer J Clin 2006;56:106-130.

2. Bostwick DG, Qian J, Bergstralh E, et al. Prediction of capsular perforation and seminal vesicle invasion in prostate cancer. J Urol $1996 ; 155: 1361-1367$.

3. Koh H, Kattan MW, Scardino PT, et al. A nomogram to predict seminal vesicle invasion by the extent and location of cancer in systematic biopsy results. J Urol 2003;170:1203-1208.

4. Masterson TA, Pettus JA, Middleton RG, Stephenson RA. Isolated seminal vesicle invasion imparts better outcomes after radical retropubic prostatectomy for clinically localized prostate cancer: prognostic stratification of pT3b disease by nodal and margin status. Urology 2005;66:152-155.

5. Ikonen S, Karkkainen P, Kivisaari L, et al. Magnetic resonance imaging of clinically localized prostatic cancer. J Urol 1998;159:915-919.

6. Ikonen S, Karkkainen P, Kivisaari L, et al. Endorectal magnetic resonance imaging of prostatic cancer: comparison between fat-suppressed T2-weighted fast spin echo and three-dimensional dual-echo, steady-state sequences. Eur Radiol 2001;11:236-241.

7. May F, Treumann T, Dettmar P, Hartung R, Breul J. Limited value of endorectal magnetic resonance imaging and transrectal ultrasonography in the staging of clinically localized prostate cancer. BJU Int 2001;87:66-69.

8. Rorvik J, Halvorsen OJ, Albrektsen G, Ersland L, Daehlin L, Haukaas S. MRI with an endorectal coil for staging of clinically localised prostate cancer prior to radical prostatectomy. Eur Radiol 1999;9:29-34. 
9. Cornud F, Flam T, Chauveinc L, et al. Extraprostatic spread of clinically localized prostate cancer: factors predictive of pT3 tumor and of positive endorectal MR imaging examination results. Radiology 2002;224:203-210.

10. Quinn DI, Henshall SM, Brenner PC, et al. Prognostic significance of preoperative factors in localized prostate carcinoma treated with radical prostatectomy: importance of percentage of biopsies that contain tumor and the presence of biopsy perineural invasion. Cancer 2003;97:1884-1893.

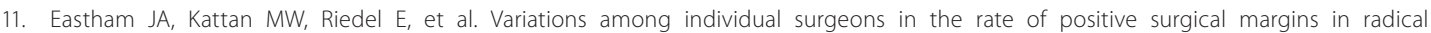
prostatectomy specimens. J Urol 2003;170:2292-2295.

12. Osborn JR, Ramsden AR, Chodak GW, Persad RA. Should the therapeutic approach to prostate cancer with seminal vesicle invasion be reviewed: improving functional results without diminishing oncological outcome? BJU Int 2004;94:482-483.

13. Zlotta AR, Djavan B, Petein M, Susani M, Marberger M, Schulman CC. Prostate specific antigen density of the transition zone for predicting pathological stage of localized prostate cancer in patients with serum prostate specific antigen less than $10 \mathrm{ng} / \mathrm{ml}$. J Urol 1998;160:20892095.

14. Perrotti M, Kaufman RP Jr, Jennings TA, et al. Endo-rectal coil magnetic resonance imaging in clinically localized prostate cancer: is it accurate? J Urol 1996;156:106-109.

15. Presti JC Jr, Hricak H, Narayan PA, Shinohara K, White S, Carroll PR. Local staging of prostatic carcinoma: comparison of transrectal sonography and endorectal MR imaging. AJR Am J Roentgenol 1996;166:103-108.

16. Bartolozzi C, Menchi I, Lencioni R, et al. Local staging of prostate carcinoma with endorectal coil MRI: correlation with whole-mount radical prostatectomy specimens. Eur Radiol 1996;6:339-345.

17. Hull GW, Rabbani F, Abbas F, Wheeler TM, Kattan MW, Scardino PT. Cancer control with radical prostatectomy alone in 1,000 consecutive patients. J Urol 2002;167:528-534.

18. Guillonneau B, Debras B, Veillon B, Bougaran J, Chambon E, Vallancien G. Indications for preoperative seminal vesicle biopsies in staging of clinically localized prostatic cancer. Eur Urol 1997;32:160-165.

19. Epstein JI, Partin AW, Potter SR, Walsh PC. Adenocarcinoma of the prostate invading the seminal vesicle: prognostic stratification based on pathologic parameters. Urology 2000;56:283-288.

20. Kattan MW, Stapleton AM, Wheeler TM, Scardino PT. Evaluation of a nomogram used to predict the pathologic stage of clinically localized prostate carcinoma. Cancer 1997;79:528-537.

21. Partin AW, Mangold LA, Lamm DM, Walsh PC, Epstein JI, Pearson JD. Contemporary update of prostate cancer staging nomograms (Partin tables) for the new millennium. Urology 2001;58:843-848.

22. Graefen M, Karakiewicz Pl, Cagiannos I, et al. Validation study of the accuracy of a postoperative nomogram for recurrence after radical prostatectomy for localized prostate cancer. J Clin Oncol 2002;20:951-956.

23. Ramsden AR, Chodak G. An analysis of risk factors for biochemical progression in patients with seminal vesicle invasion: validation of Kattan's nomogram in a pathological subgroup. BJU Int 2004;93:961-964.

24. Sala E, Akin O, Moskowitz CS, et al. Endorectal MR imaging in the evaluation of seminal vesicle invasion: diagnostic accuracy and multivariate feature analysis. Radiology 2006;238:929-937.

25. Hricak $\mathrm{H}$, Wang $\mathrm{L}$, Wei $\mathrm{DC}$, et al. The role of preoperative endorectal magnetic resonance imaging in the decision regarding whether to preserve or resect neurovascular bundles during radical retropubic prostatectomy. Cancer 2004;100:2655-2663.

26. Mullerad M, Hricak H, Wang L, Chen HN, Kattan MW, Scardino PT. Prostate cancer: detection of extracapsular extension by genitourinary and general body radiologists at MR imaging. Radiology 2004;232:140-146.

27. Wang L, Mullerad M, Chen HN, et al. Prostate cancer: incremental value of endorectal MR imaging findings for prediction of extracapsular extension. Radiology 2004;232:133-139.

28. Wang L, Hricak H, Kattan M, et al. Combined endorectal and phased-array MRI in the prediction of pelvic lymph node metastasis in prostate cancer. AJR Am J Roentgenol 2006;186:743-748.

29. Efron B, Tibshirani RJ. An introduction to the bootstrap. New York, NY: Chapman \& Hall, 1993.

30. Ohori M, Scardino PT, Lapin SL, Seale-Hawkins C, Link J, Wheeler TM. The mechanisms and prognostic significance of seminal vesicle involvement by prostate cancer. Am J Surg Pathol 1993;17:1252-1261.

31. John H, Hauri D. Seminal vesicle-sparing radical prostatectomy: a novel concept to restore early urinary continence. Urology 2000;55:820824.

32. Boring CC, Squires TS, Tong T. Cancer statistics, 1992. CA Cancer J Clin 1992;42:19-38.[Free Full Text]

33. Terris MK, McNeal JE, Freiha FS, Stamey TA. Efficacy of transrectal ultrasound-guided seminal vesicle biopsies in the detection of seminal vesicle invasion by prostate cancer. J Urol 1993;149:1035-1039.

34. Bostwick DG, Qian J, Schlesinger C. Contemporary pathology of prostate cancer. Urol Clin North Am 2003;30:181-207.

35. Graefen M, Karakiewicz PI, Cagiannos I, et al. International validation of a preoperative nomogram for prostate cancer recurrence after radical prostatectomy. J Clin Oncol 2002;20:3206-3212.

36. Ravery V, Chastang C, Toublanc M, Boccon-Gibod L, Delmas V. Percentage of cancer on biopsy cores accurately predicts extracapsular extension and biochemical relapse after radical prostatectomy for T1-T2 prostate cancer. Eur Urol 2000;37:449-455.

37. Wang L, Hricak H, Kattan M, Chen H, Scardino P, Kuroiwa K. Prediction of organ-confined prostate cancer: incremental value of MR imaging and MR spectroscopic imaging to staging nomograms. Radiology 2006;238:597-603. 
5 


\section{Criptincinental Value of Multiplanar Cross-Referencing for Prostate Cancer Staging with Endorectal MRI}




\title{
Incremental Value of Multiplanar Cross-Referencing for Prostate Cancer Staging with Endorectal MRI
}

\author{
Supported by National Institutes of Health grant NIH R01 CA76423.
}

Liang Wang, Jingbo Zhang, Lawrence H. Schwartz, Halley Eisenberg, Nicole M. Ishill, Chaya S. Moskowitz, Peter Scardino and Hedvig Hricak

Departments of Radiology (L.W., J.Z., L.H.S., H.E., H.H.), Epidemiology \& Biostatistics (N.M.I., C.S.M.), and Urology (P.S.), Memorial Sloan-Kettering Cancer Center, 1275 York Ave., New York, NY 10021.

\section{Abstract}

Objective: The purpose of this study was to assess whether use of the PACS cross-referencing tool in 3D MRI improves tumor staging of prostate cancer when pathologic findings are used as the reference standard.

Materials and Methods: The institutional review board granted a waiver of informed consent for the study. Endorectal MRI at 1.5 T was performed before radical prostatectomy in 255 consecutive patients. Two radiologists unaware of the clinical data retrospectively and independently interpreted MR images without and with cross-referencing to predict the presence of extracapsular extension (ECE) and seminal vesicle invasion (SVI). Histopathologic findings were used as the reference standard. Area under the receiver operating characteristics curve (AUC), sensitivity and specificity, and weighted kappa statistics were calculated.

Results: At histologic examination, 68 (27\%) of the patients were found to have ECE and 13 (5\%) of the patients to have SVI; the latter all had ECE. In detecting ECE, both reviewers had a higher AUC using cross-referencing $(p<0.001$ for both). The weighted kappa value was 0.56 for MRI alone and 0.76 for MRI with cross-referencing, indicating fair to good interobserver agreement. Sensitivity and specificity for ECE with MRI alone and with cross-referencing were $43 \%$ and $94 \%$ and $57 \%$ and $100 \%$ for reviewer 1 and $40 \%$ and $93 \%$ and $59 \%$ and $98 \%$ for reviewer 2, respectively. In detecting SVI, both reviewers had a higher AUC with cross-referencing ( $p=0.007$ and $p=0.056$ for reviewers 1 and 2 , respectively). Reviewer 1 benefited much more from cross-referencing than did reviewer 2 . The weighted kappa statistic was 0.69 for MRI alone and the same with cross-referencing, indicating good interobserver agreement. Sensitivity and specificity for SVI with MRI alone and with cross-referencing, respectively, were $23 \%$ and $83 \%$ and $46 \%$ and $93 \%$ for reviewer 1 and 31\% and 91\% and 54\% and 95\% for reviewer 2 .

Conclusion: PACS cross-referencing significantly improves tumor staging of prostate cancer with 3D MRI. Some reviewers benefit more than others from use of this tool. 


\section{INTRODUCTION}

$\mathbf{P}$ rostate cancer affects men of all races, cultures, and ethnic backgrounds and is a major public health and economic burden in the United States and other industrialized countries $[1,2]$. In the past decade, there has been a dramatic downward trend in the stage of prostate cancer determined at diagnosis [1, 3]. Preoperative identification of extracapsular extension (ECE) and seminal vesicle invasion (SVI) is an important factor in staging and prognosis that may modify treatment decisions and treatment planning $[4,5]$. Because it improves surgical planning, identification of these characteristics increases the chances that the tumor will be resected completely with only minimal damage to the surrounding tissue so important to recovery of normal function $[6,7]$.

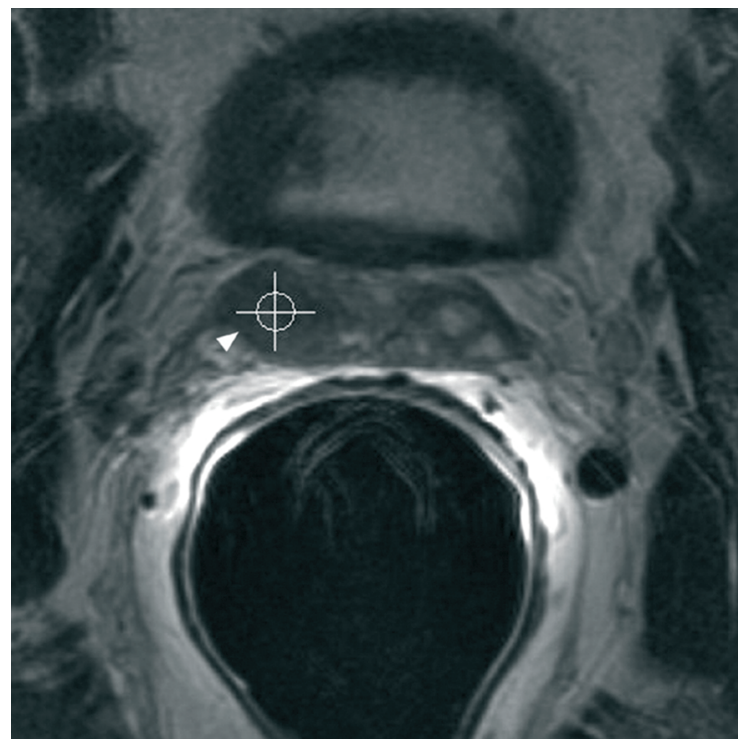

Fig. 1A: 53-year-old man with clinical stage T1c prostate carcinoma with Gleason score of $3+3$ and prostate-specific antigen level of $11.9 \mathrm{ng} / \mathrm{mL}$. MRI without PACS cross-referencing indicated possible extracapsular extension (ECE) at right base and possible right seminal vesicle invasion (SVI) (scores of 3 and 3, respectively). However, MRI with PACS cross-referencing indicated definite ECE and definite SVI (scores of 5 and 5, respectively). Axial T2-weighted image shows point of interest (crosshairs) selected by reviewer in low-signal-intensity area using cross-referencing tool. Irregular nodularity is seen in right seminal vesicle (arrowhead), indicating seminal vesicle invasion.

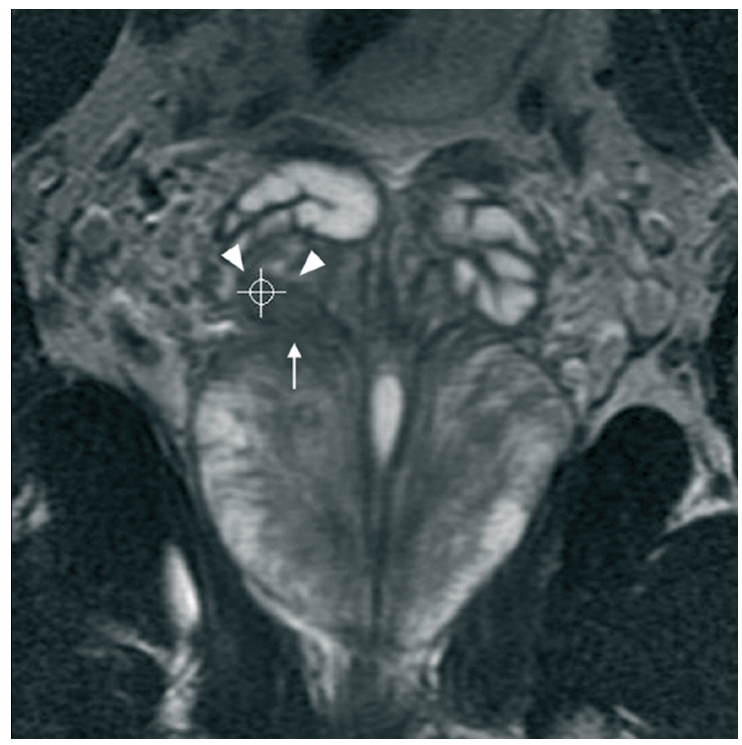

Fig. 1B: 53 -year-old man with clinical stage T1c prostate carcinoma with Gleason score of $3+3$ and prostate-specific antigen level of 11.9 $\mathrm{ng} / \mathrm{mL}$. MRI without PACS cross-referencing indicated possible extracapsular extension (ECE) at right base and possible right seminal vesicle invasion (SVI) (scores of 3 and 3, respectively). However, MRI with PACS cross-referencing indicated definite ECE and definite SVI (scores of 5 and 5 , respectively). T2-weighted coronal MR image with cross-referencing shows ECE (arrow) at right base and right seminal vesicle invasion (arrowheads). Crosshairs indicate point of interest identified by cross-referencing tool. Relationship between seminal vesicle and central zone is shown clearly. 


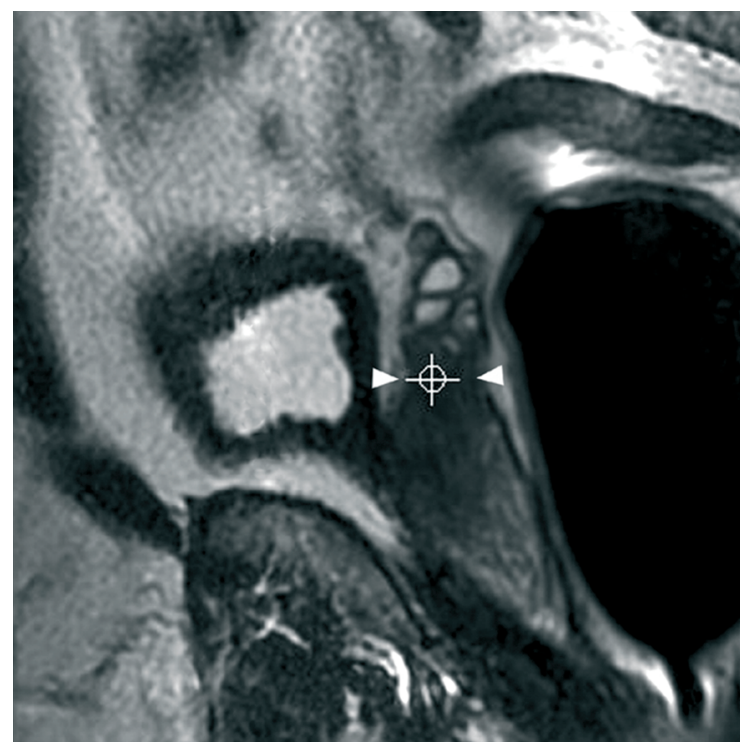

Fig. 1C: 53-year-old man with clinical stage T1c prostate carcinoma with Gleason score of $3+3$ and prostate-specific antigen level of $11.9 \mathrm{ng} / \mathrm{mL}$. MRI without PACS cross-referencing indicated possible extracapsular extension (ECE) at right base and possible right seminal vesicle invasion (SVI) (scores of 3 and 3, respectively). However, MRI with PACS cross-referencing indicated definite ECE and definite SVI (scores of 5 and 5, respectively). Sagittal T2-weighted MR image shows right seminal vesicle invasion (arrowheads). Crosshairs indicate point of interest identified by cross-referencing tool.
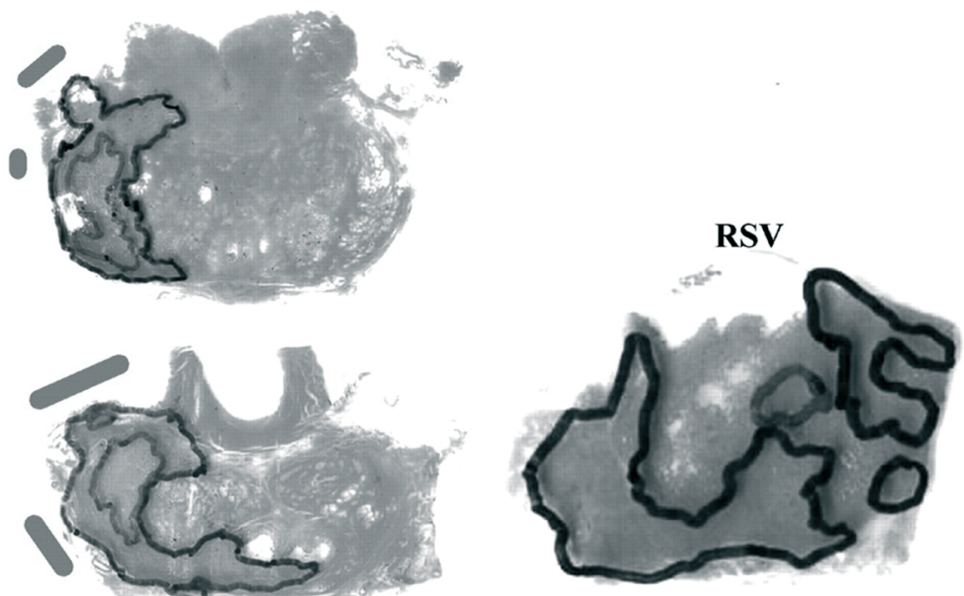

Fig. 1D: 53-year-old man with clinical stage $\mathrm{T1c}$ prostate carcinoma with Gleason score of $3+3$ and prostate-specific antigen level of $11.9 \mathrm{ng} / \mathrm{mL}$. MRI without PACS cross-referencing indicated possible extracapsular extension (ECE) at right base and possible right seminal vesicle invasion (SVI) (scores of 3 and 3 , respectively). However, MRI with PACS cross-referencing indicated definite ECE and definite SVI (scores of 5 and 5, respectively). Histologic photograph shows whole-mount sections that confirm presence of ECE (red) at right base and right seminal vesicle invasion (blue). RSV = right seminal vesicle.

(Color version available in chapter 18)

Probability of the presence of ECE and SVI currently is best determined from staging nomograms with which pathologic stage is estimated from the pretreatment level of prostate-specific antigen, clinical stage, and Gleason grade in the biopsy specimen [7-9]. However, staging nomograms are limited because they do not incorporate the results of imaging studies that could assist in prediction of ECE and SVI [7-9].

Because of high spatial resolution, superior contrast resolution, multiplanar capability, and large field of view, MRI has played an increasingly important role in prostate cancer staging. However, although it has high specificity (ECE specificity, 47-99\%; SVI specificity, 81-99\%) [10-14], MRI has widely varying sensitivity (ECE sensitivity, 22-80\%; SVI sensitivity, 34-71\%) [15-17]. Many of the difficulties in prostate cancer staging have been caused by poor understanding of the anatomic features of the prostate owing to its small size, presence of periprostatic structures [18, 19], and the partial volume effect on MRI [20]. The partial volume effect arises in volumetric images when more than one tissue type is present in a voxel. In such cases, voxel intensity depends not only on the imaging sequence and tissue properties but also on the proportion of each tissue type present in the voxel [20]. Depending on the direction and thickness of the MRI slice, the border between the tissues can become unclear. Presence of the partial volume effect can lead to false assessment of tumor capsules and tumor invasion [21]. 
PACS technology facilitates the display and distribution of digital images [22]. With a PACS, medical images obtained with various techniques, such as CT, MRI, sonography, and digital projection radiography [23], and other pertinent information are transmitted to various, sometimes remote, locations over networks. Display of the images on computer workstations for soft-copy viewing allows simultaneous consultations and almost instant reporting from radiologists working in several locations [23]. The PACS workstation (Centricity RA 1000, GE Healthcare) at our institution has a cross-referencing feature whereby selection of a voxel in any one plane highlights the corresponding voxel in the intersecting planes. This feature is also available on systems such as the Synapse PACS (Fujifilm) and iSite PACS (Philips Medical Systems). Although it may be tempting to assume that new technology will enhance radiologists' performance, this is not always the case. For example, current literature suggests that accuracy of interpretation of sonograms is similar for images interpreted on a monitor and those interpreted on film [24]. The purpose of our study was to assess whether use of PACS cross-referencing improves tumor staging of prostate cancer with 3D MRI when pathologic findings are the reference standard.

\section{Materials and Methods}

\section{Patient Characteristics}

The institutional review board granted exempt status for this retrospective, single-institution cross-sectional study with a waiver of informed consent. Patient data were collected and handled in accordance with Health Insurance Portability and Accountability Act regulations. From March 22, 2004, through January 7, 2005, 255 patients with prostate cancer were consecutively referred from the urology department to undergo endorectal MRI before radical retropubic prostatectomy performed by one of six attending surgeons at our institution. Mean patient age was 59 years (range, 40-86 years). None of the patients received neoadjuvant hormonal or radiation therapy before radical prostatectomy. All patients had a tissue diagnosis of prostate cancer based on biopsy results. Clinical serum level of prostate-specific antigen, Gleason grade at biopsy, clinical stage, greatest percentage of cancer in all biopsy cores, percentage of cores with positive results in all biopsy cores, presence of perineural invasion, and MR data were recorded retrospectively from medical records.

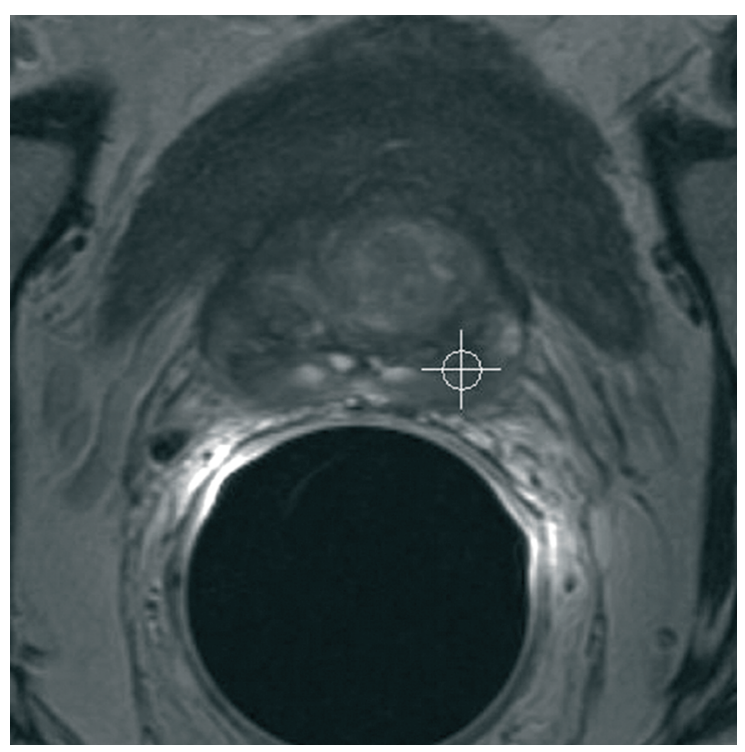

Fig. 2A: 63-year-old man with clinical stage T1c prostate carcinoma with Gleason score of $4+4$, prostate-specific antigen level of 12.9 $\mathrm{ng} / \mathrm{mL}$. MRI without PACS cross-referencing indicated possible extracapsular extension (ECE) at left base and possible left seminal vesicle invasion (SVI) (scores of 3 and 3, respectively). However, cross-referenced MR images ( $A-C$ ) indicated no ECE and no SVI (scores of 1 and 1, respectively). Axial T2-weighted MR image shows point of interest (crosshairs) identified by cross-referencing tool.

\section{MRI Technique}

Endorectal MRI was performed with a 1.5-T whole-body MRI system (Signa Horizon, GE Healthcare). Examinations were performed with the patients in the supine position, a body coil for excitation, and a pelvic phasedarray coil (GE Healthcare) in combination with a commercially available balloon-covered expandable endorectal coil (Medrad) for signal reception. T1-weighted axial and spin-echo images were obtained from the aortic bifurcation to the symphysis pubis with the following parameters: TR/TE, 700/8; slice thickness, $5 \mathrm{~mm}$; interslice 

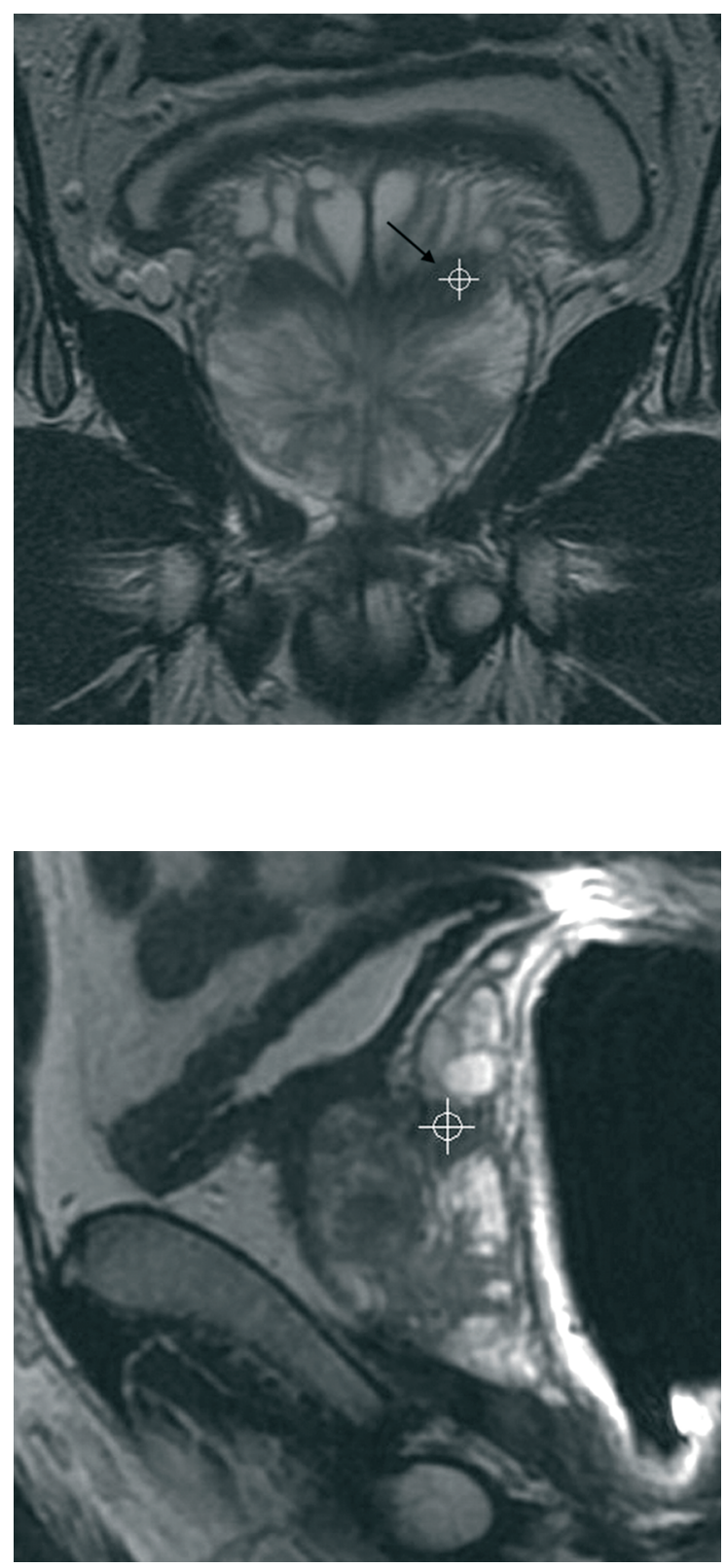

Fig. 2B: 63-year-old man with clinical stage T1c prostate carcinoma with Gleason score of $4+4$, prostate-specific antigen level of 12.9 $\mathrm{ng} / \mathrm{mL}$. MRI without PACS cross-referencing indicated possible extracapsular extension (ECE) at left base and possible left seminal vesicle invasion (SVI) (scores of 3 and 3 , respectively). However, crossreferenced MR images (A-C) indicated no ECE and no SVI (scores of 1 and 1, respectively). Coronal T2-weighted MR image shows interface (arrow) between seminal vesicle and central zone of prostate. Crosshairs indicate point of interest identified by cross-referencing tool.
Fig. 2C: 63 -year-old man with clinical stage T1c prostate carcinoma with Gleason score of $4+4$, prostate-specific antigen level of 12.9 $\mathrm{ng} / \mathrm{mL}$. MRI without PACS cross-referencing indicated possible extracapsular extension (ECE) at left base and possible left seminal vesicle invasion (SVI) (scores of 3 and 3, respectively). However, cross-referenced MR images (A-C) indicated no ECE and no SVI (scores of 1 and 1, respectively). Sagittal T2-weighted image with cross-referencing shows point of interest selected by reviewer (crosshairs).

gap, 1 mm; field of view, 24 cm; matrix size, $256 \times 192$; frequency direction, transverse (to prevent obstruction of the pelvic node by endorectal coil motion artifact); and number of excitations, one. Thin-section, high-spatial-resolution axial and coronal T2-weighted fast spin-echo images of the prostate and seminal vesicles were obtained with the following parameters: TR/effective TE, 5,000/96; echo-train length, 16; slice thickness, 3 mm; interslice gap, 0 mm; field of view, $14 \mathrm{~cm}$; matrix size, $256 \times 192$; frequency direction, anteroposterior (to prevent obstruction of the prostate by endorectal coil motion artifact); and number of excitations, 4. All MRI data were entered into the PACS of our radiology department.

\section{MRI Analysis on PACS Workstation}

MR images were retrospectively analyzed independently by two radiologists who were aware of the hypothesis being tested but unaware of the clinical, surgical, and histologic findings for the 255 patients who had undergone radical prostatectomy. Reviewer 1 had 4 years of research experience in MRI of the prostate during which 


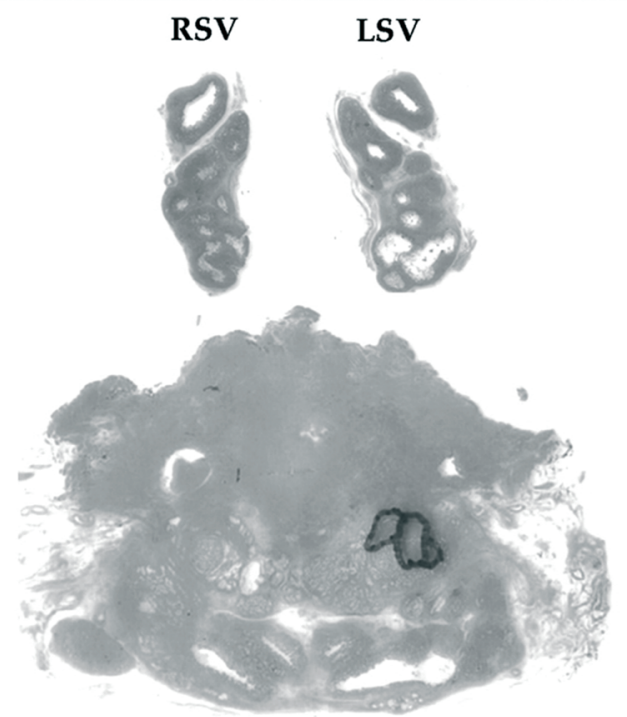

Fig. 2D: 63-year-old man with clinical stage T1c prostate carcinoma with Gleason score of $4+4$, prostate-specific antigen level of $12.9 \mathrm{ng} / \mathrm{mL}$. MRI without PACS cross-referencing indicated possible extracapsular extension (ECE) at left base and possible left seminal vesicle invasion (SVI) (scores of 3 and 3, respectively). However, cross-referenced MR images (A-C) indicated no ECE and no SVI (scores of 1 and 1, respectively). Histopathologic photograph shows whole-mount sections confirming absence of extracapsular extension and seminal vesicle invasion at left base. RSV = right seminal vesicle, LSV = left seminal vesicle.

(Color version available in chapter 18).

he had interpreted more than 1,000 prostate MR images. Reviewer 2 had completed a dedicated body-MRI fellowship and had interpreted more than 500 prostate MR images, most in a 1-year span. Criteria for signs of ECE on MR images included capsular irregularity, bulging of the capsule, capsular retraction, obliteration of the rectoprostatic angle, and asymmetry or direct involvement of the neurovascular bundles [10,25]. Criteria for SVI were a focal lowsignal-intensity mass or diffuse enlargement with low signal intensity and loss of the perceptible vesical wall on both T1- and T2-weighted sequences [25].

At the PACS workstation, the reviewers opened the examinations in the axial, coronal, and sagittal image series and assigned separate scores for the probabilities of ECE and SVI on a scale of 1-5 (1, definitely absent; 2, probably absent; 3 , possibly present; 4 , probably present; 5 , definitely present). These scores were designated MRI without PACS crossreferencing. The reviewers then activated the cross-referencing feature. With this feature, when a reviewer selects a point of interest (or voxel) in any one plane (i.e., image series), an image in each intersecting plane is automatically displayed with a target symbol (or crosshairs) on the corresponding voxel. After using the cross-referencing tool, the radiologists assigned scores for ECE and SVI, again using the 5-point scale. These scores were designated MRI with PACS cross-referencing (Figs. 1A, 1B, 1C, 1D, 2A, 2B, 2C, and 2D).

\section{Pathologic Assessment and Comparison with MR Images}

Core biopsy specimens were evaluated for Gleason grade, greatest percentage of cancer in all biopsy cores, percentage of cores with positive findings in all biopsy cores, and presence of perineural invasion. All prostatectomy specimens were inked with India ink tattoo dye (green dye on right, blue dye on left) and fixed in 10\% formalin for 36 hours. The distal $5 \mathrm{~mm}$ of the apex was amputated and coned. The rest of the gland was serially sectioned from apex to base for acquisition of axial slices at 3-mm intervals (axial step sections) and submitted in entirety for paraffin embedding as whole mounts. The seminal vesicles were amputated and submitted separately. After paraffin embedding, microsections were placed on glass slides and stained with $\mathrm{H}$ and $\mathrm{E}$. The axial pathologic sections were numbered consecutively from apex to base, and the cancerous areas were mapped in each section with marker. Pathologic stage and surgical Gleason score were determined for each patient. ECE was defined as invasion of prostate cancer beyond the prostate capsule into the periprostatic soft tissue. SVI was defined as invasion of prostate cancer cells into the seminal vesicles. Presence or absence of ECE and SVI was recorded from the surgical pathology report. 


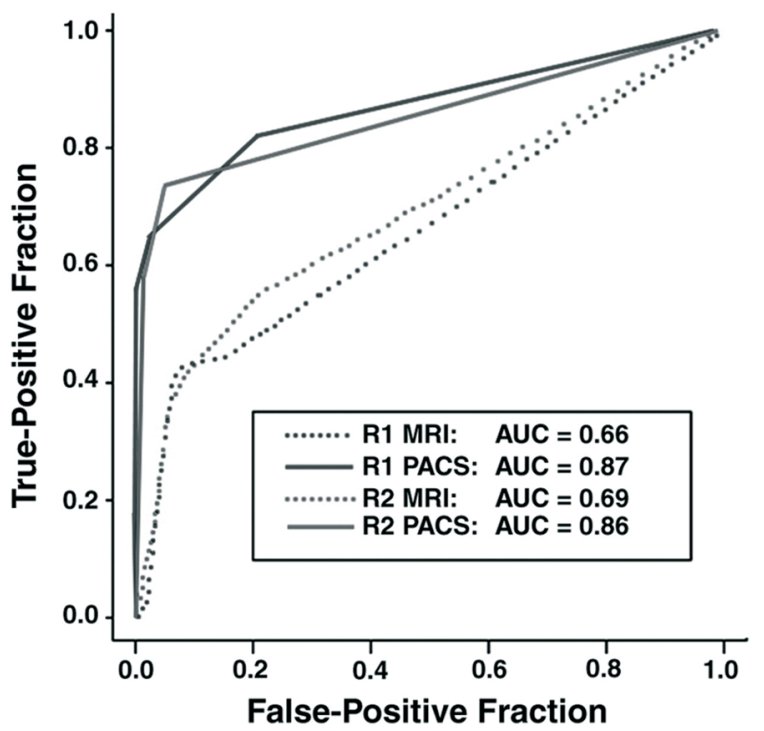

Fig. 3: Graph shows results of receiver operating characteristic analysis for detection of extracapsular extension with MRI alone and MRI with PACS cross-referencing. $\mathrm{R} 1$ = reviewer $1, \mathrm{R} 2$ = reviewer 2, MRI = MRI alone, PACS = MRI with PACS cross-referencing, AUC = area under curve.

(Color version available in chapter 18).

\section{Statistical Analysis}

Two of the authors were responsible for statistical analysis. Empirically estimated receiver operating characteristics (ROC) curves were used to evaluate the radiologists' accuracy in detecting SVI and ECE on MRI. For each ROC curve, the area under the curve (AUC) and the corresponding confidence intervals (CI) were estimated with nonparametric methods. Comparisons of the AUCs of a single reviewer were conducted with methods described by DeLong et al. [26]. To assess interobserver variability in determining the presence of ECE and SVI on MRI alone and MRI with PACS cross-referencing, we used a weighted kappa statistic with weights $1-|i-j| /(5-1)$ where $i, j=1, \ldots, 5$ denotes the rating categories for the first and second reviewers [27]. Analyses were performed with Intercooled Stata 8.0 for Windows (Stata) and S-Plus for Windows 6.2.1 (Insightful).

\section{RESULTS}

\section{Surgical Histopathologic Findings}

At histopathologic examination, 68 (27\%) of 255 patients had ECE and 13 (5\%) had SVI. All 13 patients with SVI had ECE.

\section{MRI Findings}

Detection of ECE-ROC curves for reviewers 1 and 2 in detecting and localizing ECE are plotted in Figure 3. Reviewer 1 had an AUC of 0.66 (95\% Cl, 0.58-0.73) using MRI alone and an AUC of 0.87 (95\% Cl, 0.82-0.93) using MRI with PACS cross-referencing. Reviewer 1 performed significantly better using MRI with PACS cross-referencing than using MRI alone ( $p<0.001)$. Reviewer 2 had an AUC of 0.69 (95\% Cl, 0.61-0.77) using MRI alone and an AUC of 0.86 (95\% Cl, 0.79-0.92) using MRI with PACS cross-referencing. Reviewer 2 also performed significantly better using MRI with PACS cross-referencing $(p<0.001)$. For detection of ECE with MRI alone, the weighted kappa was 0.56 , and for detection of ECE with MRI and PACS cross-referencing, it was 0.76 , indicating fair to good agreement between the two reviewers.

We dichotomized the 5 -point scoring system and used 4 cut points to assess the sensitivity and specificity of MRI in the diagnosis of ECE (Table 1). When cut point 3 was chosen (so that values 1-3 indicated absence of ECE and values 4 and 5 indicated presence of ECE) for reviewer 1, sensitivity and specificity were $43 \%$ and $94 \%$ for MRI alone and $57 \%$ and $100 \%$ for MRI with PACS cross-referencing. At the same cut point for reviewer 2, sensitivity and specificity were $40 \%$ and $93 \%$ for MRI alone and $59 \%$ and $98 \%$ for MRI with PACS cross-referencing. 
Table 1: Sensitivity and Specificity for Detection of Extracapsular Extension at 4 Cut Points of the 5-Point Scoring System

\begin{tabular}{|c|c|c|c|c|}
\hline & $\begin{array}{c}\text { Cut Point } 1 \\
(1=\text { No; } 2-5=\text { Yes })\end{array}$ & $\begin{array}{c}\text { Cut Point } 2( \\
1,2=\text { No; } 3-5=\text { Yes })\end{array}$ & $\begin{array}{c}\text { Cut Point } 3 \\
(1-3=\text { No; } 4,5=\text { Yes })\end{array}$ & $\begin{array}{c}\text { Cut Point } 4 \\
(1-4=\text { No; } 5=\text { Yes })\end{array}$ \\
\hline \multicolumn{5}{|l|}{ Reviewer 1} \\
\hline \multicolumn{5}{|l|}{ MRI alone } \\
\hline Sensitivity & 51 & 44 & 43 & 3 \\
\hline Specificity & 73 & 85 & 94 & 98 \\
\hline \multicolumn{5}{|l|}{ MRI + PACS } \\
\hline Sensitivity & 82 & 68 & 57 & 43 \\
\hline Specificity & 79 & 95 & 100 & 100 \\
\hline \multicolumn{5}{|l|}{ Reviewer 2} \\
\hline \multicolumn{5}{|l|}{ MRI alone } \\
\hline Sensitivity & 69 & 56 & 40 & 10 \\
\hline Specificity & 53 & 78 & 93 & 98 \\
\hline \multicolumn{5}{|l|}{$M R I+P A C S$} \\
\hline Sensitivity & 82 & 74 & 59 & 41 \\
\hline Specificity & 65 & 95 & 98 & 99 \\
\hline
\end{tabular}

Note-All values are percentages. MRI + PACS = MRI with PACS cross-referencing

Detection of SVI-ROC curves for reviewers 1 and 2 in detecting and localizing SVI are plotted in Figure 4. Reviewer 1 had an AUC of 0.62 (95\% Cl, 0.48-0.76) using MRI alone and an AUC of $0.87(95 \% \mathrm{Cl}, 0.77-0.96)$ using MRI with PACS cross-referencing. Reviewer 1 performed significantly better using PACS cross-referencing ( $p=0.007)$. Reviewer 2 had an AUC of $0.73(95 \% \mathrm{Cl}, 0.58-0.88)$ using MRI alone and an AUC of $0.90(95 \% \mathrm{Cl}, 0.80-0.99)$ using MRI with PACS cross-referencing. Reviewer 2 also performed better using PACS cross-referencing, although for reviewer 2 the difference had only borderline significance $(p=0.056)$. In the detection of SVI, the weighted kappa statistic was 0.69 for both MRI alone and MRI with PACS cross-referencing, indicating good interobserver agreement.

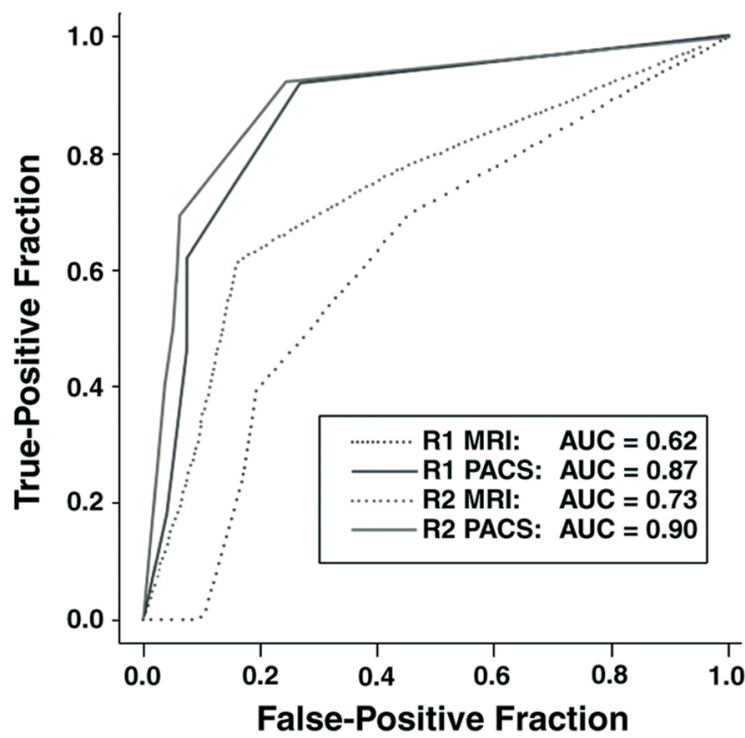

Fig. 4: Graph shows results of receiver operating characteristic analysis for detection of seminal vesicle invasion with MRI with PACS cross-referencing and MRI alone. R1 = reviewer $1 ; \mathrm{R} 2=$ reviewer $2 ; \mathrm{MRI}=\mathrm{MRI}$ alone; $\mathrm{PACS}=\mathrm{MRI}$ with PACS cross-referencing, $\mathrm{AUC}=$ area under curve.

(Color version available in chapter 18). 
We dichotomized the 5 -point scoring system and used 4 cut points to assess the sensitivity and specificity of MRI in the diagnosis of SVI (Table 2). When cut point 3 was used (so that values 1-3 indicated absence of SVI and values 4 and 5 indicated presence of SVI) for reviewer 1, sensitivity and specificity were $23 \%$ and $83 \%$ for MRI alone and $46 \%$ and $93 \%$ for MRI with PACS cross-referencing. At the same cut point for reviewer 2, sensitivity and specificity were $31 \%$ and $91 \%$ for MRI alone and 54\% and 95\% for MRI with PACS cross-referencing.

Table 2: Sensitivity and Specificity for Seminal Vesicle Invasion at 4 Cut Points of the 5-Point Scoring System

\begin{tabular}{|c|c|c|c|c|}
\hline & $\begin{array}{c}\text { Cut Point } 1 \\
(1=\text { No; } 2-5=\text { Yes })\end{array}$ & $\begin{array}{c}\text { Cut Point } 2( \\
1,2=\text { No; } 3-5=\text { Yes) }\end{array}$ & $\begin{array}{c}\text { Cut Point } 3 \\
(1-3=\text { No; } 4,5=\text { Yes })\end{array}$ & $\begin{array}{c}\text { Cut Point } 4 \\
(1-4=\text { No; } 5=\text { Yes })\end{array}$ \\
\hline \multicolumn{5}{|l|}{ Reviewer 1} \\
\hline \multicolumn{5}{|l|}{ MRI alone } \\
\hline Sensitivity & 69 & 38 & 23 & 0 \\
\hline Specificity & 55 & 81 & 83 & 90 \\
\hline \multicolumn{5}{|l|}{$M R I+P A C S$} \\
\hline Sensitivity & 92 & 62 & 46 & 23 \\
\hline Specificity & 73 & 93 & 93 & 95 \\
\hline \multicolumn{5}{|l|}{ Reviewer 2} \\
\hline \multicolumn{5}{|l|}{ MRI alone } \\
\hline Sensitivity & 77 & 62 & 31 & 15 \\
\hline Specificity & 57 & 84 & 91 & 95 \\
\hline \multicolumn{5}{|l|}{$\mathrm{MRI}+\mathrm{PACS}$} \\
\hline Sensitivity & 92 & 69 & 54 & 31 \\
\hline Specificity & 76 & 94 & 95 & 98 \\
\hline
\end{tabular}

Note-All values are percentages. MRI + PACS = MRI with PACS cross-referencing

\section{Discussion}

The Surveillance, Epidemiology, and End Results (SEER) program of the National Cancer Institute has shown a dramatic down-staging of prostate cancer at diagnosis over the last 2 decades. SEER data show that from 1995 to 2000, 90\% of cases of prostate cancer were at a local or regional stage at diagnosis, compared with 72\% from 1983 to 1987 $[1,3]$. In our patient population, 27\% of the patients had evidence of ECE and 5\% had evidence of SVI at surgical histopathologic examination. These results are consistent with SEER data [2].

ECE and SVI are important prognostic factors for recurrence after radical prostatectomy $[4,28]$. ECE is associated with greater risk of a positive surgical margin, which further decreases the chance of long-term cancer control $[4,7]$. SVI is associated with an increased incidence of lymph node metastasis and a worse prognosis, even in the absence of lymph node involvement [29]. Accurate determination of the presence of ECE and SVI before treatment may alter treatment selection and planning $[4,7,9]$.

MRI is generally considered the most accurate imaging method for local staging of prostate cancer [30-32]. Engelbrecht et al. [14] conducted a meta-analysis of 146 studies performed from January 1984 to May 2000 and described in 71 articles and five abstracts. The findings showed that MRI had an AUC of $0.60 \pm 0.19$ (SD) in detection of ECE and an AUC of $0.62 \pm 0.13$ in detection of SVI. The role of MRI in prostate cancer management has been changing with the development of techniques such as endorectal MRI, MR spectroscopic imaging, and PACS data storage $[6,10-12,25,33,34]$. With a PACS, medical information can be stored, recalled, displayed, manipulated, and printed digitally. Use of a PACS simplifies workflow, enhances productivity, and makes information accessible to multiple users simultaneously. Examination findings digitally stored in a secure computer system can be quickly transmitted to 
referring physicians, who in some cases can view the images in their offices via computer. Improvements in patient care include shorter hospital stays, decreased waiting times, faster diagnoses, and protection of personal medical information.

Our study showed that in the detection of ECE and SVI, radiologists performed substantially better using MRI with PACS cross-referencing than with MRI alone. PACS cross-referencing is particularly helpful in displaying the relationship between the seminal vesicles and the central zone of the prostate (Figs. 2A, 2B, 2C, and 2D). In our study, one reviewer benefited more from cross-referencing than did the other. This finding may relate to inherent differences in the way people see things.

One limitation of our study was that cross-referencing immediately followed the initial image review, so conclusions made at the initial review were incorporated into the final review. Thus there was almost no way that the reviewers could perform worse with cross-referencing than without it. Another limitation was that ECE and SVI were analyzed patient by patient but not according to specific location (anterior, lateral, posterior or apex, middle, base), so the ECE and SVI predicted were not conclusively and specifically located.

The initial sensitivities (43\% and 40\%) and AUC (0.66-0.69) of the reviewers for ECE in our study were relatively low for a major medical center with experienced reviewers [14]. The sensitivities (57\% and 59\%) and AUCs (0.87 and 0.86) after cross-referencing are more in line with expected findings. Both reviewers use the cross-referencing tool when routinely interpreting prostate MRI examinations. Therefore it is possible that when they were required not to use this tool at initial review, their accuracy suffered. It is also possible that the results were affected by anticipatory bias; that is, the radiologists may have expected better results with cross-referencing and held back in their initial interpretations without consciously intending to do so. In addition, the results may have been influenced by verification bias because the reviewers were aware that all patients had undergone surgical treatment, which was a decision influenced by initial MRI results.

The fact that all patients in this study underwent surgery implies that they had relatively low stages of local disease. Our study showed that cross-referencing on a PACS improved detection of relatively subtle ECE and SVI in these patients. In addition, the use of cross-referencing improved interobserver agreement in the detection of ECE. To support our findings and in light of the study limitations, we recommend further prospective multicenter confirmatory studies involving more reviewers with more varied experience.

In summary, our findings suggest that the PACS cross-referencing tool allows radiologists to more accurately interpret MR images of the prostate, significantly improving tumor staging of prostate cancer with MRI. Some reviewers benefit more than others from use of this tool.

\section{References}

1. Jemal A, Murray T, Ward E, et al. Cancer statistics, 2005. CA Cancer J Clin2005; 55:10 -30

2. American Cancer Society. Cancer facts \& figures 2005. Atlanta, GA: American Cancer Society, 2005. Publication no. 5008.05

3. Boring CC, Squires TS, Tong T. Cancer statistics, 1993. CA Cancer J Clin1993; 43:7 -26

4. Hull GW, Rabbani F, Abbas F, Wheeler TM, Kattan MW, Scardino PT. Cancer control with radical prostatectomy alone in 1,000 consecutive patients. J Urol 2002;167:528-534

5. Catalona WJ, Ramos CG, Carvalhal GF. Contemporary results of anatomic radical prostatectomy. CA Cancer J Clin1999; $49: 282$-296

6. Hricak H, Wang L, Wei DC, et al. The role of preoperative endorectal magnetic resonance imaging in the decision regarding whether to preserve or resect neurovascular bundles during radical retropubic prostatectomy. Cancer2004; 100:2655 -2663

7. Ohori M, Kattan MW, Koh H, et al. Predicting the presence and side of extracapsular extension: a nomogram for staging prostate cancer. J Urol 2004;171:1844-1849

8. Partin AW, Mangold LA, Lamm DM, Walsh PC, Epstein Jl, Pearson JD. Contemporary update of prostate cancer staging nomograms (Partin tables) for the new millennium. Urology2001; 58:843-848

9. Koh H, Kattan MW, Scardino PT, et al. A nomogram to predict seminal vesicle invasion by the extent and location of cancer in systematic biopsy results. J Urol2003; 170:1203-1208

10. Wang L, Mullerad M, Chen HN, et al. Prostate cancer: incremental value of endorectal MR imaging findings for prediction of extracapsular extension. Radiology2004; 232:133-139

11. Mullerad M, Hricak H, Wang L, Chen HN, Kattan MW, Scardino PT. Prostate cancer: detection of extracapsular extension by genitourinary and general body radiologists at MR imaging. Radiology2004; 232:140 -146

12. Cornud F, Flam T, Chauveinc L, et al. Extraprostatic spread of clinically localized prostate cancer: factors predictive of pT3 tumor and of positive endorectal MR imaging examination results. Radiology2002; 224:203 -210

13. Rajesh A, Coakley FV. MR imaging and MR spectroscopic imaging of prostate cancer. Magn Reson Imaging Clin N Am2004; $12: 557$-579 
14. Engelbrecht MR, Jager GJ, Laheij RJ, Verbeek AL, van Lier HJ, Barentsz JO. Local staging of prostate cancer using magnetic resonance imaging: a metaanalysis. Eur Radiol2002; 12:2294 -2302

15. Wallner K. MR imaging for prostate cancer staging: beauty or beast? Int J Radiat Oncol Biol Phys2002; 52:886 -887

16. Sonnad SS, Langlotz CP, Schwartz JS. Accuracy of MR imaging for staging prostate cancer: a metaanalysis to examine the effect of technologic change. Acad Radiol2001; 8:149-157

17. May F, Treumann T, Dettmar P, Hartung R, Breul J. Limited value of endorectal magnetic resonance imaging and transrectal ultrasonography in the staging of clinically localized prostate cancer. BJU Int 2001;87:66 -69

18. Gray H, Lewis WH. Anatomy of the human body, 20th ed. Available at: www.bartleby.com. Accessed August 31, 2005

19. Coakley FV, Hricak H. Radiologic anatomy of the prostate gland: a clinical approach. Radiol Clin North Am2000; $38: 15$-30

20. Gonzalez Ballester MA, Zisserman AP, Brady M. Estimation of the partial volume effect in MRI. Med Image Anal2002; 6:389-405

21. Maeda M, Suzuki E, Yoshiya K, et al. Partial volume effect in MRI: a phantom study [in Japanese]. Nippon Igaku Hoshasen Gakkai Zasshi 1989;49:1404-1410

22. Miyamoto K, Abe S, Kawakami Y. Picture archiving and communication system in Hokkaido University Hospital: advantage and disadvantage of HU-PACS chest roentgenogram images in the outpatient clinic. J Digit Imaging 1991;4:28 -31

23. De Backer Al, Mortele KJ, De Keulenaer BL. Picture archiving and communication system. Part 1. Filmless radiology and distance radiology. JBR-BTR 2004;87:234 -241

24. Hertzberg BS, Kliewer MA, Paulson EK, et al. PACS in sonography: accuracy of interpretation using film compared with monitor displaypicture archiving and communication systems. AJR 1999;173:1175 -1179

25. Claus FG, Hricak H, Hattery RR. Pretreatment evaluation of prostate cancer: role of MR imaging and $1 \mathrm{H}$ MR spectroscopy. RadioGraphics2004; 24[suppl 1]:S167 -S180

26. DeLong ER, DeLong DM, Clarke-Pearson DL. Comparing the areas under two or more correlated receiver operating characteristic curves: a nonparametric approach. Biometrics1988; 44:837-845

27. Cicchetti DV, Fleiss JL. A new procedure for assessing reliability if scoring EEG sleep recordings. Am J EEG Technol1977; 11:101 -109

28. D'Amico AV, Renshaw AA, Sussman B, Chen MH. Pretreatment PSA velocity and risk of death from prostate cancer following external beam radiation therapy. JAMA2005; 294:440-447

29. Epstein J, Partin AW, Potter SR, Walsh PC. Adenocarcinoma of the prostate invading the seminal vesicle: prognostic stratification based on pathologic parameters. Urology2000; 56:283-288

30. Ikonen S, Karkkainen P, Kivisaari L, et al. Endorectal magnetic resonance imaging of prostatic cancer: comparison between fat-suppressed T2-weighted fast spin echo and three-dimensional dual-echo, steady-state sequences. Eur Radiol2001; 11:236 -241

31. Rorvik J, Halvorsen OJ, Albrektsen G, Ersland L, Daehlin L, Haukaas S. MRI with an endorectal coil for staging of clinically localised prostate cancer prior to radical prostatectomy. Eur Radiol1999; 9:29-34

32. Tempany CM. MR staging of prostate cancer: how we can improve our accuracy with decisions aids and optimal techniques. Magn Reson Imaging Clin N Am 1996;4:519-532

33. Futterer JJ, Scheenen TW, Huisman HJ, et al. Initial experience of 3 tesla endorectal coil magnetic resonance imaging and $1 \mathrm{H}$-spectroscopic imaging of the prostate. Invest Radiol2004; 39:671 -680

34. Sosna J, Pedrosa I, Dewolf WC, Mahallati H, Lenkinski RE, Rofsky NM. MR imaging of the prostate at 3 Tesla: comparison of an external phased-array coil to imaging with an endorectal coil at 1.5 Tesla. Acad Radiol2004; 11:857-862 
6 


\section{Combined Endorectal and Phased-Array MRI in the Prediction of Pelvic Lymph Node Metastasis in Prostate Cancer}




\section{Combined Endorectal and Phased-Array MRI in the Prediction of Pelvic Lymph Node Metastasis in Pros- tate Cancer \\ Supported by National Institutes of Health grant IRGICA76423-0IRI.}

Liang Wang, Hedvig Hricak, Michael W. Kattan, Lawrence H. Schwartz, Steven C. Eberhardt, Hui-Ni Chen and Peter T. Scardino

Department of Radiology, Memorial Sloan-Kettering Cancer Center, 1275 York Ave., Rm. C-278, New York, NY 10021, Department of Urology, Sidney Kimmel Center for Prostate and Urologic Cancers, Memorial Sloan-Kettering Cancer Center, New York, NY 10021.

Received October 28, 2004; accepted after revision February 3, 2005.

Presented at the 2004 American Roentgen Ray Society annual meeting, Miami Beach, FL.

\section{Abstract}

Objective: The objectives of our study were to evaluate the accuracy of combined endorectal and phased-array MRI in detecting pelvic lymph node metastasis (LNM) in patients with prostate cancer and to determine whether radiologists' predictions of LNM improve with the incorporation of Partin nomogram or MRI findings (or both) regarding extracapsular extension or seminal vesicle invasion.

Subjects and Methods: Between May 1999 and September 2003, 411 consecutive patients with clinically localized prostate cancer underwent MRI before surgery. Serum prostate-specific antigen (PSA) level, Gleason grade, clinical stage, greatest percentage of cancer and percentage of positive cores in all biopsy cores, presence of perineural invasion on biopsy, and likelihood of LNM based on the Partin tables (2001 version) were recorded. MRI studies were interpreted prospectively, but the risks of LNM, extracapsular extension, and seminal vesicle invasion were scored retrospectively on the basis of the MRI reports. Surgical pathology constituted the standard of reference. The accuracy of LNM prediction was assessed using areas under receiver operating characteristic curves (AUCs) and univariate and multivariate logistic regression analyses. For multivariate models, the jackknife method was used for bias correction. A p value below 0.05 denoted statistical significance.

Results: At surgical pathology, LNM was present in 22 (5\%) of 411 patients. MRI was an independent statistically significant predictor of LNM ( $p=0.002)$, with positive and negative predictive values of $50 \%$ and $96.36 \%$, respectively. On multivariate analysis, prediction of lymph node status using the model that included all MRI variables (extracapsular extension, seminal vesicle invasion, and LNM) along with the Partin nomogram results had a significantly greater AUC than the univariate model that included only MRI LNM findings ( $A U C=0.892$ vs 0.633 , respectively; $P$ $<0.01)$.

Conclusion: Incorporation of the Partin nomogram results and MRI findings regarding both extracapsular extension and seminal vesicle invasion improves the MR prediction of LNM in patients with prostate cancer. 


\section{INTRODUCTION}

rostate cancer is the most common cancer and the second leading cause of cancer death in American men. The American Cancer Society estimated that in 2005 in the United States, 232,090 new cases of prostate cancer would be diagnosed and 39,350 men would die of the disease [1]. In a patient with newly diagnosed prostate cancer, the presence of lymphatic metastases is an important prognostic factor, indicating great risk for progression to established distant metastases and death [2-5]. The risk of metastatic disease at 10 years is $31 \% \pm$ $7 \%$ (mean \pm SD) for patients with negative nodes compared with $83 \% \pm 7 \%$ for patients with positive nodes at the time of initial treatment. The risk of dying of prostate cancer is $17 \% \pm 6 \%$ at 10 years for patients with negative nodes compared with $57 \% \pm 11 \%$ for patients with positive nodes [4]. Accurate diagnosis of lymph node metastasis (LNM) is essential in treatment selection and planning.

Since the late 1980s, studies have consistently shown that MRI has limited value in the assessment of LNM in patients with prostate cancer [6-14] (Table 1). Although MRI provides images with excellent anatomic detail for the evaluation of locoregional disease and has high specificity for LNM, the sensitivity of MRI for the detection of LNM is relatively low [15-17]. Furthermore, the National Cancer Institute's Surveillance, Epidemiology, and End Results Program has shown that there has been a dramatic downstaging of prostate cancer at the time of diagnosis [1, 18]. This change has made the MRI evaluation of lymph nodes even more difficult. Not only has the incidence of LNM declined steeply (in a study by Soh et al. [19], it decreased from 23\% in 1984 to 2\% in 1995), but cancerous nodes, when present, are likely to be smaller [1, 2, 18, 19]. A recent study by Harisinghani et al. [14] showed that high-resolution MRI with lymphotropic superparamagnetic nanoparticles allows the detection of small and otherwise undetectable LNMs in patients with prostate cancer. Although these results are excellent and promising, the low incidence of LNM raises the question of whether lymphotropic superparamagnetic nanoparticles should be used routinely. Although the U.S. Food and Drug Administration (FDA) is expected to approve the clinical use of lymphotropic superparamagnetic nanoparticles, the question of patient selection remains unanswered.

In part, the limitations of imaging in the evaluation of LNM in patients with prostate cancer have been offset by the development of Partin staging nomograms or tables (available online as supplemental data), which predict the final pathologic stage, including the presence of LNM, on the basis of clinical stage, serum prostate-specific antigen (PSA) level, and Gleason grade [20, 21]. The Partin staging nomograms are a validated predictive instrument widely used for patient counseling [22-25]. However, as a treatment planning tool, they are limited by the fact that they do not incorporate anatomic data that could assist in interventions aimed to control the disease.

We undertook this study to investigate the role of combined endorectal and phased-array MRI in nodal metastasis detection in a representative sample of the current prostate cancer patient population, with its low incidence of LNM. Our goal was to assess the accuracy of MRI in predicting prostate cancer LNM. In addition, we aimed to determine whether the prediction of LNM improved with the inclusion of MRI findings regarding extracapsular extension or seminal vesicle invasion (or both) or with the use of the Partin nomograms. 
Table 1: Literature Review Summary: Studies That Used Pathologic Correlation to Evaluate the Prediction of Prostate Cancer Lymph Node Metastasis (LNM) by MRI

\begin{tabular}{|c|c|c|c|c|c|c|c|c|c|}
\hline $\begin{array}{l}\text { Author(s) } \\
\text { [reference no.] }\end{array}$ & $\begin{array}{c}\text { No. of } \\
\text { Patients }^{a}\end{array}$ & $\%$ LNM & $\begin{array}{l}\text { Name (location) } \\
\text { of Institution(s) }\end{array}$ & $\begin{array}{l}\text { Year of } \\
\text { Study }\end{array}$ & $\begin{array}{c}\text { Sensitivity } \\
(\%)\end{array}$ & $\begin{array}{l}\text { Specificity } \\
(\%)\end{array}$ & $\begin{array}{l}\text { PPV } \\
\text { (\%) }\end{array}$ & $\begin{array}{l}\text { NPV } \\
(\%))\end{array}$ & $\begin{array}{c}\text { Accuracy } \\
(\%)\end{array}$ \\
\hline Bezzi et al. [6] & 51 & $\begin{array}{c}25 \\
(13 \text { of } 51)\end{array}$ & $\begin{array}{c}\text { University of } \\
\text { Pennsylvania } \\
\text { (Philadelphia,PA) }\end{array}$ & 1988 & 69 (9 of 13) & 95 (36 of 38 ) & & & 88 \\
\hline Beer et al. [7] & 24 & & $\begin{array}{c}\text { Ludwig- } \\
\text { Maximilians- } \\
\text { Universitat (Munchen, } \\
\text { Germany) }\end{array}$ & 1989 & 26 & & & & \\
\hline Kier et al. [8] & 27 & & $\begin{array}{c}\text { Yale University } \\
\text { School of Medicine } \\
\text { (New Haven, CT) }\end{array}$ & 1993 & & & & & 85 \\
\hline Nicolas et al. [9] & 392 & & $\begin{array}{l}\text { Universitats- } \\
\text { Krankenhaus } \\
\text { (Hamburg- } \\
\text { Eppendorf, Germany) }\end{array}$ & 1994 & 54.4 & & & & \\
\hline Jager et al. [10] & 63 & $\begin{array}{c}24 \\
(15 \text { of } 63)\end{array}$ & $\begin{array}{l}\text { University Hospital } \\
\text { (Nijmegen, } \\
\text { The Netherlands) }\end{array}$ & 1996 & $60(9$ of 15$)$ & 98 (47 of 48$)$ & 90 & 95.99 & 89 \\
\hline Perrotti et al. [11] & 56 & & $\begin{array}{c}\text { Albany Medical } \\
\text { College (Albany, NY) }\end{array}$ & 1996 & 0 & 91 & 0 & 94 & 86 \\
\hline Tuzel et al. [12] & 17 & $\begin{array}{c}0 \\
(0 \text { of } 17)\end{array}$ & $\begin{array}{l}\text { Dokuz Eylül University, } \\
\text { School of Medicine } \\
\text { Inciralti (Izmir, Turkey) }\end{array}$ & 1998 & 0 & 93 & & & 88.2 \\
\hline Borley et al.b [13] & 42 & $\begin{array}{c}26 \\
(11 \text { of } 42)\end{array}$ & $\begin{array}{c}\text { King's College } \\
\text { Hospital (London, UK) }\end{array}$ & 2003 & 27.3 (3 of 11) & & & & \\
\hline $\begin{array}{l}\text { Harisinghani et al. } \\
\text { c [14] }\end{array}$ & 80 & $\begin{array}{c}41 \\
\text { (33 of } 80)\end{array}$ & $\begin{array}{l}\text { Massachusetts General } \\
\text { Hospital and Harvard } \\
\text { Medical School (Boston, } \\
\text { MA) and University } \\
\text { Medical Center (Nijmegen, } \\
\text { The Netherlands) }\end{array}$ & 2003 & $\begin{array}{c}45.4(\mathrm{MRI}) \\
100(\mathrm{MRI} \\
\text { with LSN) }\end{array}$ & $\begin{array}{l}78.7(\mathrm{MRI}) \\
95.7(\mathrm{MRI} \\
\text { with LSN) }\end{array}$ & $\begin{array}{l}60 \text { (MRI); } \\
94.2(\mathrm{MRI} \\
\text { with LSN) }\end{array}$ & $\begin{array}{c}67.2(\mathrm{MRI}) \\
100(\mathrm{MRI} \\
\text { with LSN) }\end{array}$ & \\
\hline $\begin{array}{l}\text { Current study } \\
\text { (Wang et al.) }\end{array}$ & 411 & $\begin{array}{c}5 \\
\text { (22 of } 411)\end{array}$ & $\begin{array}{c}\text { Memorial Sloan-Kettering } \\
\text { Cancer Center } \\
\text { (New York, NY) }\end{array}$ & 2004 & 27.27 & 98.46 & 50.00 & 96.36 & \\
\hline
\end{tabular}

Note-PPV = positive predictive value, NPV = negative predictive value, LSN = lymphotropic superparamagnetic nanoparticles.

a Numbers in this column represent patients in each study who had pathologically proven prostate cancer and correlation of lymph node status on pathology to MRI findings

${ }^{\mathrm{b}}$ High-risk patients

c With LSN

\section{Subjects And Methods}

\section{Patients}

Between November 1999 and September 2003, 411 consecutive patients with clinically localized prostate cancer underwent MRI before radical retropubic prostatectomy and pelvic lymphadenectomy (Table 2). For all patients, the surgery was performed at our institution. The mean patient age was 57.6 years (range, 32-74 years). None of the patients received neoadjuvant hormonal or radiation therapy before surgery. All patients had tissue diagnosis of prostate cancer on biopsy specimens. The approval of the institutional review board was obtained, and all patients gave informed consent. Patient accrual was done as part of an ongoing National Cancer Institute trial, and some of the cases have been previously reported in studies on the locoregional evaluation of prostate cancer by MRI [26-28]. 
Table 2: Distribution of Preoperative Clinical Variables

\begin{tabular}{|c|c|c|c|c|c|}
\hline Variable & No. of Patients & Mean & Range & SD & $\%$ \\
\hline Serum PSA & 411 & 7.68 & $0.74 \approx 113.4$ & 8.90 & \\
\hline \multicolumn{6}{|l|}{ Gleason grade } \\
\hline $3+2$ & 2 & & & & 0.49 \\
\hline $3+3$ & 262 & & & & 63.75 \\
\hline $3+4$ & 87 & & & & 21.17 \\
\hline $3+5$ & 1 & & & & 0.24 \\
\hline $4+3$ & 27 & & & & 6.57 \\
\hline $4+4$ & 23 & & & & 5.60 \\
\hline $4+4$ or $3+5$ & 1 & & & & 1.24 \\
\hline $4+5$ & 7 & & & & 1.70 \\
\hline $5+5$ & 1 & & & & 0.24 \\
\hline $\begin{array}{l}\text { Greatest } \% \text { of } \\
\text { cancer in all biopsy cores }\end{array}$ & 409 & 0.33 & $0.01 \approx 1$ & 0.2711 & \\
\hline $\begin{array}{l}\% \text { of positive cores } \\
\text { in all biopsy cores }\end{array}$ & 377 & 0.40 & $0.04 \approx 1$ & 0.2669 & \\
\hline \multicolumn{6}{|l|}{$\begin{array}{l}\text { Presence of perineural } \\
\text { invasion on biopsy }\end{array}$} \\
\hline Positive & 54 & & & & 86.86 \\
\hline Negative & 357 & & & & 13.14 \\
\hline \multicolumn{6}{|l|}{ Clinical stage } \\
\hline $\mathrm{T} 1 \mathrm{c}$ & 237 & & & & 57.80 \\
\hline $\mathrm{T} 2 \mathrm{a}$ & 95 & & & & 23.17 \\
\hline $\mathrm{T} 2 \mathrm{~b}$ & 41 & & & & 10.00 \\
\hline $\mathrm{T} 2 \mathrm{C}$ & 37 & & & & 9.02 \\
\hline $\mathrm{T} 3, \mathrm{~T} 4, \mathrm{~N}+, \mathrm{M}+$ & 0 & & & & 0 \\
\hline
\end{tabular}

The following clinical variables were recorded for each patient: clinical stage, pretreatment serum PSA, biopsy Gleason sum, greatest percentage of cancer in all biopsy cores, percentage of positive cores in all biopsy cores, and presence of perineural invasion. Clinical stage was assigned using the 1992 American Joint Committee on Cancer (AJCC) and the Union Internationale Contre le Cancer (UICC) TNM guidelines based on digital rectal examination regardless of the results of sonography or other imaging techniques. Serum PSA was measured using a Hybritech assay (Hybritech Tandem-R, Beckman Coulter). Central pathologic review was not performed, but all specimens were analyzed by dedicated genitourinary pathologists at our institution.

\section{Combined Endorectal and Phased-Array MRI Data Acquisition and Interpretation}

MRI was performed at 1.5 T with state-of-the-art imaging systems (Signa Horizon, GE Healthcare) and pelvic phasedarray and endorectal coils. Images of the pelvis, extending from the pubic symphysis to the level of the aortic bifurcation, were obtained. The MR pulse sequences consisted of axial spin-echo T1-weighted images with the following parameters: TR/TE, 700/8; slice thickness, 5 mm; interslice gap, 1 mm; field of view, 24 cm; matrix, 256 x 192; and 1 excitation. Thin-section high-spatial-resolution axial, coronal, and sagittal T2-weighted fast spin-echo images of 
the prostate and seminal vesicles were obtained with the following parameters: TR/TE, 5,000/96; echo-train length, 16; slice thickness, 3 mm; interslice gap, 0 mm; field of view, 14-20 cm; matrix, 256 × 192; and 3 excitations. Axial T2weighted images were postprocessed to correct for the reception profile of the endorectal coil.

MRI studies were prospectively interpreted by 11 MR body radiologists during their regular clinical assignment to the MR service. Each radiologist made his or her determination regarding the presence of LNM based on his or her practice pattern and knowledge of previously described MRI features of LNM. As a basic clinical guideline, the radiologists classified lymph nodes on MRI as malignant if the short-axis diameter was elongated and exceeded $10 \mathrm{~mm}$ or was rounded and exceeded $8 \mathrm{~mm}$, according to the standard accepted criteria [10]. On the basis of the radiologists' written reports, a single observer retrospectively and separately scored the probabilities of LNM, extracapsular extension, and seminal vesicle invasion using the following rating scale: 5 , definite yes; 4, probable yes; 3 , possible yes; 2 , probable no; and 1, definite no [14].

\section{Partin Staging Nomogram}

Based on serum PSA, biopsy Gleason grade, and clinical staging, the likelihood of LNM according to the 2001 version of the Partin staging nomograms was recorded [21].

\section{Histology}

The histology reports from the core biopsies were all recorded at our institution and included the following: Gleason grade, greatest percentage of cancer in all biopsy cores, percentage of positive cores in all biopsy cores, and presence of perineural invasion. Radical prostatectomy specimens were examined as previously described by Yossepowitch et al. [29]. Standard template pelvic lymph node dissection was performed, encompassing all nodal tissue from the medial inferior margin of the external iliac vein down to the internal iliac and obturator vessels. Step-section histopathology findings were used as the standard of reference for extracapsular extension, seminal vesicle invasion, and LNM. Surgical margin status, pelvic lymph node status, presence of seminal vesicle invasion, and extracapsular extension were recorded.

\section{Statistical Analysis}

Both univariate and multivariate analyses were performed for all clinical and imaging variables to predict LNM. We evaluated the area under the receiver operating characteristic (ROC) curve for each variable. A model combining extracapsular extension and seminal vesicle invasion on MRI with the prediction of nodal disease and a model for the addition of the Partin nomogram to MRI were constructed; the areas under the ROC curves (AUCs) were calculated and compared [30]. For the multivariate models, the jackknife method, a form of resampling that reduces the optimistic bias, was used to obtain the bias-corrected probabilities and construct the ROC curves. A p value of less than 0.05 was considered significant. The sensitivity, specificity, negative predictive value, positive predictive value, and accuracy of MRI for the detection of LNM were determined, with positive LNM on MRI defined by a score of 3-5. Software programs used for data analysis were SAS, version 8.2 (SAS Institute), and S-PLUS, version 2000 (Insightful).

\section{RESULTS}

On surgical histopathology, positive lymph nodes were found in 22 (5\%) of 411 patients. Table 2 summarizes the preoperative distribution of the clinical variables, and Table 3 shows the distribution of final pathologic staging [31]. Performance characteristics for each variable are listed in Table 4. In the evaluation of LNM, MRI had sensitivity and specificity of $27.27 \%$ and $98.46 \%$, respectively, and positive predictive value and negative predictive value of $50 \%$ and $95.99 \%$, respectively. 
Table 3: Distribution of Pathologic Staging According to the American Joint Committee on Cancer [31]

\begin{tabular}{lc} 
Final Pathologic Stage & No. (\%) of Patients \\
\hline T1c & $2(0.49)$ \\
\hline T2a & $76(18.49)$ \\
\hline T2b & $220(53.53)$ \\
\hline T2c & $1(0.24)$ \\
\hline T3a & $73(17.76)$ \\
\hline T3b & $26(6.32)$ \\
\hline T4 & $13(3.16)$ \\
\hline N1 & $22(5.35)$ \\
\hline
\end{tabular}

\section{Table 4: Performance Characteristics at Select Cut Points for Predicting Lymph Node Metastasis}

\begin{tabular}{|c|c|c|c|c|c|}
\hline Preoperative Variable & Cut Points & Sensitivity (\%) & Specificity (\%) & PPV (\%) & NPV (\%) \\
\hline Serum PSA & $0-10$ vs $\geq 10.1$ & $50.00(11 / 22)$ & $85.60(333 ? 389)$ & $16.42(11 / 67)$ & $96.80(333 / 344)$ \\
\hline Gleason grade & $\begin{array}{c}3+2,3+3 \\
3+4 \text { vs } 3+5 \\
4+3,4+4 \\
4+5,5+5\end{array}$ & $54.55(12 / 22)$ & $87.66(341 / 389)$ & $20.00(12 / 60)$ & $97.15(341 / 351)$ \\
\hline Clinical stage & T1c vs T2a, b, c & $72.73(16 / 22)$ & $59.54(231 / 388)$ & $9.25(16 / 173)$ & $97.47(231 / 237)$ \\
\hline $\begin{array}{l}\text { Greatest \% of cancer in } \\
\text { all biopsy scores }\end{array}$ & $0-60$ vs $\geq 60$ & $63.64(14 / 22)$ & $86.56(335 / 387)$ & $21.21(14 / 66)$ & $97.67(285 / 295)$ \\
\hline $\begin{array}{l}\% \text { of positive cores in all } \\
\text { biopsy cores }\end{array}$ & $0-60 v s \geq 60$ & $54.55(12 / 22)$ & $80.28(285 / 355)$ & $14.63(12 / 82)$ & $96.61(285 / 295)$ \\
\hline $\begin{array}{l}\text { Presence of perineural } \\
\text { invasion on biopsy }\end{array}$ & $\begin{array}{l}\text { Positive vs } \\
\text { negative }\end{array}$ & $40.91(9 / 22)$ & $88.43(344 / 389)$ & $16.67(9 / 54)$ & $96.36(344 / 357)$ \\
\hline $\begin{array}{l}\text { Combined endorectal } \\
\text { and phased-array MRI }\end{array}$ & $1-2$ vs $3-5$ & $27.27(6 / 22)$ & $98.46(383 / 389)$ & $50.00(6 / 12)$ & $95.99(383 / 399)$ \\
\hline
\end{tabular}

Note- $P P V=$ positive predictive value, NPV = negative predictive value, PSA = prostate-specific antigen.

Univariate analysis (Table 5) showed that all variables were associated with LNM. In the prediction of LNM, the AUC for MRI was 0.633. In multivariate analysis, MRI $(p=0.002)$, Gleason score $(p=0.007)$, greatest percentage of cancer in all biopsy cores $(p=0.007)$, and PSA ( $p=0.004)$ were significant predictors of LNM (Table 6).

In the prediction of LNM, each of the multivariate models that incorporated MRI findings regarding extracapsular extension and seminal vesicle invasion with MRI LNM findings had a greater AUC than the univariate model that included only MRI LNM findings (Fig. 1). The model that included all MRI variables (extracapsular extension, seminal vesicle invasion, and LNM) along with the Partin nomogram results had a significantly greater AUC than the univariate model that included only MRI LNM findings (Fig. 1; Table 7: model I vs model A, 0.892 vs 0.633, respectively; $\mathrm{p}<$ 0.01). 
Table 5: Preoperative Prediction of Lymph Node Metastasis: Univariate Analysis

\begin{tabular}{lcc} 
Preoperative Variable & $P$ & Area Under ROC Curve \\
\hline Gleason grade & $<0.001$ & 0.852 \\
\hline Greatest percentage of cancer in all biopsy cores & $<0.001$ & 0.844 \\
\hline PSA & 0.002 & 0.699 \\
\hline$\%$ of positive cores in all biopsy cores & 0.006 & 0.688 \\
\hline Clinical stage & 0.005 & 0.662 \\
\hline Presence of perineural invasion on biopsy & $<0.001$ & 0.647 \\
\hline Combined endorectal and phased-array MRI & $<0.001$ & 0.633
\end{tabular}

Note- $\mathrm{ROC}=$ receiver operating characteristic, PSA = prostate-specific antigen.

Table 6: Preoperative Prediction of Lymph Node Metastasis: Multivariate Analysis

\begin{tabular}{ll} 
Preoperative Variable & $P$ \\
\hline Combined phased-array and endorectal MRI & 0.002 \\
\hline Gleason grade & 0.007 \\
\hline Greatest \% of cancer in all biopsy cores & 0.007 \\
\hline$\%$ of positive cores in all biopsy cores & 0.006 \\
\hline PSA & 0.004 \\
\hline Presence of perineural invasion on biopsy & 0.442 \\
\hline$\%$ of positive cores in all biopsy cores & 0.819 \\
\hline
\end{tabular}

Note-PSA = prostate-specific antigen .

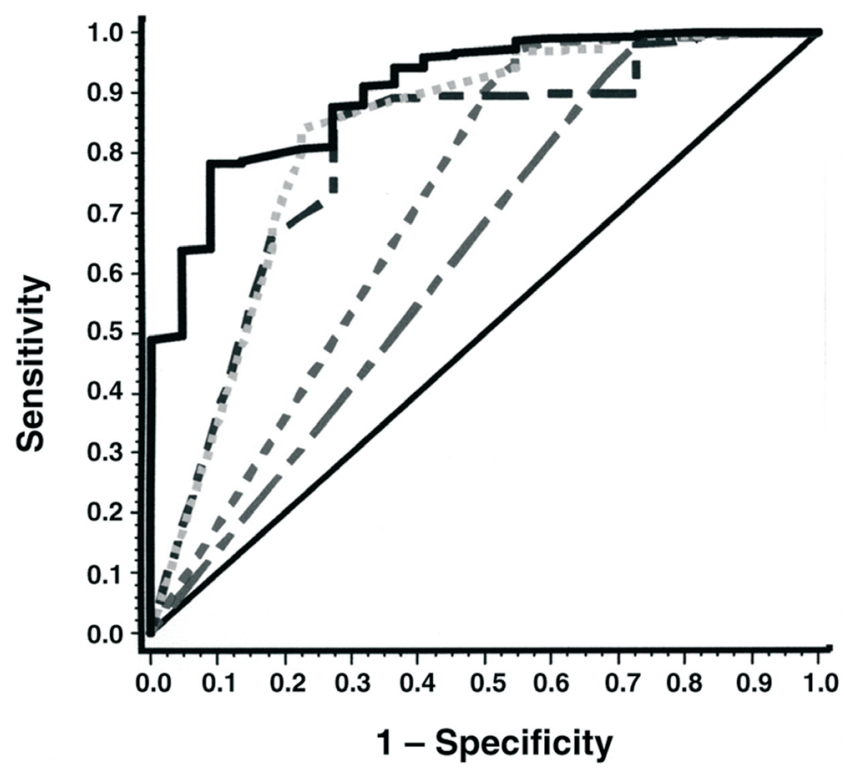

Fig. 1: Graph shows that each multivariate model had greater area under receiver operating characteristic curve (AUC) than model A (green) (Table 7), which included only MRI findings for lymph node metastasis (LMN). Model combining MRI findings for seminal vesicle invasion and LNM (model F) (red) had greater AUC than model A: 0.714 vs 0.633 , respectively $(p=0.08$ ). Model combining MRI findings for extracapsular extension and LNM (model G) (blue) had greater AUC than model A: 0.798 vs 0.633 , respectively $(p=0.15)$. Model combining MRI findings for extracapsular extension, seminal vesicle invasion, and LNM (model H) (yellow) had greater AUC than model A: 0.813 vs 0.633 , respectively $(p=0.11)$. Model combining MRI findings for extracapsular extension, seminal vesicle invasion, and LNM and Partin tomogram prediction of LNM (model I) (black) had significantly greater AUC than model A: 0.892 vs 0.633 , respectively $(p<0.01)$.

(Color version available in chapter 18 ). 
Table 7: Univariate and Multivariate Analyses: Assessing Factors Predicting Lymph Node Metastasis (LNM)

\begin{tabular}{|c|c|c|c|}
\hline Model & Type of Analysis & $P$ & Area under ROC Curve \\
\hline Model A & Univariate & & 0.633 \\
\hline Probability of $L M N{ }^{a}$ based on MRI report & & $<0.0001$ & \\
\hline Model B & Univariate & & 0.639 \\
\hline Probability of seminal vesicle invasiona based on MRI report & & $<0.0001$ & \\
\hline Model C & Univariate & & 0.770 \\
\hline Probability of extracapsular extensiona based on MRI report & & $<0.0001$ & \\
\hline Model D & Univariate & & 0.899 \\
\hline Probability of LNM according to the Partin nomograms ${ }^{b}$ & & $<0.0001$ & \\
\hline Model E & Multivariate & & $0.772^{c}$ \\
\hline Probability of extracapsular extensiona based on MRI report & & 0.0002 & \\
\hline Probability of seminal vesicle invasiona based on MRI report & & 0.0004 & \\
\hline Model F & Multivariate & & $0.714^{c}$ \\
\hline Probability of seminal vesicle invasiona based on MRI report & & $<0.0001$ & \\
\hline Probability of LMNa based on MRI report & & 0.0001 & \\
\hline Model G & Multivariate & & $0.798^{c}$ \\
\hline Probability of extracapsular extensiona based on MRI report & & $<0.0001$ & \\
\hline Probability of LMNa based on MRI report & & 0.0001 & \\
\hline Model H & Multivariate & & $0.813^{c}$ \\
\hline Probability of extracapsular extensiona based on MRI report & & 0.0012 & \\
\hline Probability of seminal vesicle invasiona based on MRI report & & 0.0027 & \\
\hline Probability of LMNa based on MRI report & & 0.0004 & \\
\hline Model I & Multivariate & & $0.892^{c}$ \\
\hline Probability of extracapsular extensiona based on MRI report & & & 0.0239 \\
\hline Probability of seminal vesicle invasiona based on MRI report & & & 0.0229 \\
\hline Probability of LMNa based on MRI report & & & 0.0110 \\
\hline Probability of LNM according to the Partin nomograms ${ }^{b}$ & & & 0.0005 \\
\hline
\end{tabular}

\section{Discussion}

Pretreatment knowledge of prostate cancer LNM is important for patient counseling and appropriate treatment selection and planning. The presence of LNMs at the time of prostate cancer diagnosis is associated with a high probability of progression after treatment and a poor prognosis $[2,4,32]$.

Over the years, studies examining the association between a number of clinical variables and the prediction of LNM have been performed. The variables studied included serum PSA, Gleason grade, clinical stage, greatest percentage of cancer in all biopsy cores, percentage of positive cores in all biopsy cores, and perineural invasion [21, 
33, 34]. Since the introduction of the Partin staging nomograms in 1993, investigators have repeatedly validated their capacity to predict LNM of clinically localized prostate cancer [22, 24, 25]. In a study by Cagiannos et al. [2] the overall predictive accuracy of the Partin tables as measured by AUC was 0.74 , while the AUCs for the three- and fourvariable nomograms developed by Cagiannos et al. were 0.76 and 0.78 , respectively $[2,20]$. In our study, the Partin nomograms-derived probability of LNM had an AUC of 0.899 .

Given the strength of the Partin nomograms, what place, if any, does MRI have in the presurgical evaluation of prostate cancer and in lymph node staging in particular? The routine use of MRI for presurgical evaluation of prostate cancer is controversial because the high cost of the test might place a substantial burden on the health care system unless its use prevents unnecessary surgery or improves treatment planning and outcomes [35]. MRI has been shown to have incremental value in prostate cancer staging and treatment planning $[26,27]$. MRI has also shown high specificity for LNM detection; however, the low sensitivity of MRI (0-69\%) in detecting LNMs has been an obstacle to its widespread use. Table 1 is a summary of the results of the major studies on the use of MRI for prostate cancer LNM staging that have used surgical pathology as the standard of reference [6, 12].

The low sensitivity of MRI has been attributed mainly to the inability of cross-sectional imaging to detect metastases in normal-sized nodes $[13,14]$. This limitation is especially important to consider in deciding when to use MRI in the current patient population because the incidence of LNM at diagnosis of prostate cancer has declined steeply and nodal metastases, when present, are often within normal-sized nodes. Tables 2 and 3 reflect the effects of prostate cancer downstaging on our study population, which was greater than the patient populations of all the other studies that used surgical pathology as the standard of reference (Table 1). Our overall positive lymph node rate of 5\% confirms the decreasing incidence of LNM in prostate cancer patients [1, 2]. Therefore, our study should permit a contemporary assessment of the potential role of MRI in the current patient population.

Our study shows that the sensitivity of MRI for nodal metastases (27.27\%) has not improved compared with the historical results [6, 12] (Table 1). However, we have also shown that in the prediction of LNM, a model that included the Partin nomograms with MRI findings regarding LNM, extracapsular extension, and seminal vesicle invasion had a significantly greater AUC (0.892) than a model that included only MRI LNM findings (AUC $=0.633 ; p<0.01$ ).

A study by Harisinghani et al. [14] showed that MRI with lymphotropic superparamagnetic nanoparticles has both high sensitivity (100\%) and high specificity (95.7\%) in detecting LNM. Although these findings are promising, it would be helpful to have a means of determining which patients are most likely to benefit from the use of nanoparticles, because the incidence of LNM at the time of radical prostatectomy in current studies is only $5 \%$ or less $[2,27$, $28,36]$. Our data confirm that MRI has high negative predictive value for LNM and indicate that a combination of endorectal and phased-array MRI with the Partin tables has high accuracy in predicting LNM. Furthermore, MRI provides anatomic information that is useful for treatment planning. We therefore suggest that combined endorectal and phased-array MRI be used in conjunction with the Partin tables in determining whether imaging with lymphotropic superparamagnetic nanoparticles is warranted.

\section{SUMmARY}

The combination of Partin nomograms and MRI findings regarding extracapsular extension, seminal vesicle invasion, and LNM offers high predictive value for LNM. Combined endorectal and phased-array MRI supplemented by the Partin nomograms could potentially be used to determine whether additional imaging with lymphotropic superparamagnetic nanoparticles is indicated. 


\section{REFERENCES}

1. American Cancer Society. Cancer facts \& figures 2005. Atlanta, GA: American Cancer Society, 2005. Report no. 5008.05

2. Cagiannos I, Karakiewicz P, Eastham JA, et al. A preoperative nomogram identifying decreased risk of positive pelvic lymph nodes in patients with prostate cancer. J Urol 2003;170:1798-1803

3. Partin AW, Pound CR, Clemens JQ, Epstein JI, Walsh PC. Serum PSA after anatomic radical prostatectomy: the Johns Hopkins experience after 10 years. Urol Clin North Am 1993;20: 713-725

4. Gervasi LA, Mata J, Easley JD, et al. Prognostic significance of lymph nodal metastases in prostate cancer. J Urol1989; 142(2 Pt 1):332 -336

5. Hricak H, Schoder H, Pucar D, et al. Advances in imaging in the postoperative patient with a rising prostate-specific antigen level. Semin Oncol 2003;30:616-634

6. Bezzi M, Kressel HY, Allen KS, et al. Prostatic carcinoma: staging with MR imaging at 1.5 T. Radiology 1988;169: 339-346

7. Beer M, Schmidt H, Riedl R. The clinical value of preoperative staging of bladder and prostatic cancers with nuclear magnetic resonance and computerized tomography [in German]. Urologe A1989; 28:65-69

8. Kier R, Wain S, Troiano R. Fast spin-echo MR images of the pelvis obtained with a phased-array coil: value in localizing and staging prostatic carcinoma. AJR 1993;161:601-606

9. Nicolas V, Beese M, Keulers A, Bressel M, Kastendieck H, Huland H. MR tomography in prostatic carcinoma: comparison of conventional and endorectal MRT [in German]. Rofo 1994;161: 319-326

10. Jager GJ, Barentsz JO, Oosterhof GO, Witjes JA, Ruijs SJ. Pelvic adenopathy in prostatic and urinary bladder carcinoma: MR imaging with a three-dimensional TI-weighted magnetization-prepared-rapid gradient-echo sequence. AJR 1996;167: 1503-1507

11. Perrotti M, Kaufman RP Jr, Jennings TA, et al. Endo-rectal coil magnetic resonance imaging in clinically localized prostate cancer: is it accurate? J Urol 1996;156: 106-109

12. Tuzel E, Sevinc M, Obuz F, Sade M, Kirkali Z. Is magnetic resonance imaging necessary in the staging of prostate cancer? Urol Int 1998; $61: 227-231$

13. Borley N, Fabrin K, Sriprasad S, et al. Laparoscopic pelvic lymph node dissection allows significantly more accurate staging in "high-risk" prostate cancer compared to MRI or CT. Scand J Urol Nephrol 2003;37 : 382-386

14. Harisinghani MG, Barentsz J, Hahn PF, et al. Noninvasive detection of clinically occult lymph-node metastases in prostate cancer. N Engl J Med 2003; 348:2491 -2499

15. Hricak H, Dooms GC, Jeffrey RB, et al. Prostatic carcinoma: staging by clinical assessment, CT, and MR imaging. Radiology1987; 162:331 $-336$

16. Wolf JS Jr, Cher M, Dall'era M, Presti JC Jr, Hricak H, Carroll PR. The use and accuracy of cross-sectional imaging and fine needle aspiration cytology for detection of pelvic lymph node metastases before radical prostatectomy. J Urol 1995;153 (3 Pt 2):993 -999

17. Tempany C, McNeil BJ. Advances in biomedical imaging. JAMA 2001; 285:562 -567

18. Boring CC, Squires TS, Tong T, Montgomery S. Cancer statistics, 1994. CA Cancer J Clin 1994;44: 7-26

19. Soh S, Kattan MW, Berkman S, Wheeler TM, Scardino PT. Has there been a recent shift in the pathological features and prognosis of patients treated with radical prostatectomy? J Urol1997; 157:2212-2218

20. Partin AW, Kattan MW, Subong EN, et al. Combination of prostate-specific antigen, clinical stage, and Gleason score to predict pathological stage of localized prostate cancer: a multi-institutional update. JAMA 1997; 277:1445 -1451

21. Partin AW, Mangold LA, Lamm DM, Walsh PC, Epstein Jl, Pearson JD. Contemporary update of prostate cancer staging nomograms (Partin tables) for the new millennium. Urology 2001;58:843-848

22. Blute ML, Bergstralh EJ, Partin AW, et al. Validation of Partin tables for predicting pathological stage of clinically localized prostate cancer. J Urol 2000;164: 1591-1595

23. Augustin H, Eggert T, Wenske S, et al. Comparison of accuracy between the Partin tables of 1997 and 2001 to predict final pathological stage in clinically localized prostate cancer. J Urol2004; 171:177 -181

24. Graefen M, Augustin $\mathrm{H}$, Karakiewicz Pl, et al. Can predictive models for prostate cancer patients derived in the United States of America be utilized in European patients? A validation study of the Partin tables. Eur Urol 2003; 43:6 -11

25. Graefen M, Karakiewicz PI, Cagiannos I, et al. Validation study of the accuracy of a postoperative nomogram for recurrence after radical prostatectomy for localized prostate cancer. J Clin Oncol 2002; 20:951 -956

26. Hricak $\mathrm{H}$, Wang $\mathrm{L}$, Wei $\mathrm{DC}$, et al. The role of preoperative endorectal magnetic resonance imaging in the decision regarding whether to preserve or resect the neurovascular bundles during radical retropubic prostatectomy. Cancer 2004;100:2655-2663

27. Wang L, Mullerad M, Chen HN, et al. Prostate cancer: incremental value of endorectal MR imaging findings for prediction of extracapsular extension. Radiology 2004;232: 133-139

28. Mullerad M, Hricak H, Wang L, Chen HN, Kattan MW, Scardino PT. Prostate cancer: detection of extracapsular extension by genitourinary and general body radiologists at MR imaging. Radiology2004; 232:140-146

29. Yossepowitch O, Sircar K, Scardino PT, et al. Bladder neck involvement in pathological stage pT4 radical prostatectomy specimens is not an independent prognostic factor. J Urol2002; 168:2011 -2015

30. Kattan MW. Judging new markers by their ability to improve predictive accuracy. J Natl Cancer Inst2003; 95:634-635

31. Fleming ID, Cooper JS, Henson DE, et al. (American Joint Committee on Cancer), eds. Cancer staging manual, 5th ed. Philadelphia, PA: Lippincott-Raven, 1997

32. Parker CC, Dearnaley DP. Radical radiotherapy for prostate cancer. Cancer Treat Rev 2003:29: 161-169

33. Graefen M, Karakiewicz PI, Cagiannos I, et al. International validation of a preoperative nomogram for prostate cancer recurrence after radical prostatectomy. J Clin Oncol 2002;20:3206-3212

34. Vargas SO, Jiroutek M, Welch WR, Nucci MR, D'Amico AV, Renshaw AA. Perineural invasion in prostate needle biopsy specimens: correlation with extraprostatic extension at resection. Am J Clin Pathol 1999; 111:223 -228

35. Penson DF, Litwin MS, Aaronson NK. Health related quality of life in men with prostate cancer. J Urol 2003;169: 1653-1661

36. Cheng L, Bergstralh EJ, Cheville JC, et al. Cancer volume of lymph node metastasis predicts progression in prostate cancer. Am J Surg Pathol 1998; 22:1491-1500 
The Role of Preoperative Endorectal Magnetic Resonance Imaging in the Decision Regarding Whether to Preserve or Resect Neurovascular Bundles During Radical Retropubic Prostatectomy 


\title{
The Role of Preoperative Endorectal Magnetic Resonance Imaging in the Decision Regarding Whether to Preserve or Resect Neurovascular Bundles During Radical Retropubic Prostatectomy
}

\author{
Supported by National Institutes of Health grant R01 CA76423
}

Hedvig Hricak, Liang Wang, David C. Wei, Fergus V. Coakley, Oguz Akin, Victor E. Reuter, Mithat Gonen, Michael W. Kattan, Chinyere N. Onyebuchi, Peter T. Scardino

Department of Radiology, Memorial Sloan-Kettering Cancer Center, New York, New York, Department of Radiology, University of California at San Francisco, San Francisco, California, Department of Epidemiology and Biostatistics, Memorial Sloan-Kettering Cancer Center, New York, New York

Received: 18 February 2004; Revised: 26 March 2004; Accepted: 31 March 2004

\section{Abstract}

Background: Because the recovery of erectile function and the avoidance of positive surgical margins are important but competing outcomes, the decision to preserve or resect a neurovascular bundle (NVB) during radical prostatectomy (RP) should be based on the most accurate information concerning the location and extent of the tumor. In the current study, the authors determined the incremental value of endorectal magnetic resonance imaging (eMRI) in making this decision.

Methods: eMRI was performed in 135 patients preoperatively. For each NVB, tumor extension to the NVB and the need for NVB resection was judged by a surgeon on a scale from 1 (definite preservation) to 5 (definite resection) before and after reviewing eMRI with a radiologist. Histopathologic findings were used as the standard of reference. The value of eMRI was assessed using binormal receiver operating characteristic (ROC) analysis adjusted for multiple observations per patient, and a mixed effects ordinal regression model was used for risk stratification.

Results: Histopathologic examination determined that NVB resection was warranted in 44 of 270 NVBs (16\%) because of posterolateral extracapsular extension ( $n=29)$, positive surgical margins $(n=7)$, or both $(n=8)$. The areas under the ROC curves (AUC) were 0.741 for pre-MRI and 0.832 for post-MRI surgical planning $(P<0.01)$. MRI findings suggested altering the surgical plan in 39\% of NVBs (106 of 270 NVBs). When the surgeon judged that the NVB resection was definitely not necessary (165 NVBs), MRI confirmed that decision in 138 NVBs (84\%); the concordant decision was correct in $96 \%$ of the cases (133 of 138 NVBs). In 36 high-risk patients ( $\geq 75 \%$ probability of extracapsular extension), MRI findings changed the surgical plan for 28 NVBs (78\%); the change was found to be appropriate in 26 cases (93\%).

Conclusions: MRI was found to significantly improve the surgeon's decision to preserve or resect the NVB during radical prostatectomy.

(c) 2004 American Cancer Society. 


\section{INTRODUCTION}

$\mathbf{R}$

adical retropubic prostatectomy (RP) provides excellent long-term disease control for patients with clinically localized prostate carcinoma.[1] Erectile dysfunction is one of the possible complications after RP. Recovery of erectile function after RP is quantitatively related to the preservation of the neurovascular bundles (NVBs). [2] However, prostate carcinoma most commonly arises in the peripheral zone of the prostate, often posteriorly, just beneath the capsule. Among patients with extracapsular extension (ECE), the tumor is most often present posterolaterally in the region of the NVBs.[3-5] Therefore, if ECE is present in the area of the NVB, preservation of an NVB may compromise disease control and result in a positive surgical margin.[4][5] A positive surgical margin reportedly increases the probability of disease recurrence.[1][5] Consequently, surgeons often resect widely in areas of suspected ECE to avoid a positive surgical margin.[4][6][7] In spite of all precautions, positive surgical margins are still present in approximately 23-31\% of RP specimens.[4][7]

The presence and location of ECE is difficult to predict from clinical stage, serum prostate-specific antigen (PSA) lev$\mathrm{el}$, and the biopsy Gleason grade, even when quantitative assessment of the extent of tumor in systematic biopsies is considered.[8] Previous studies of endorectal magnetic resonance imaging (eMRI) compared the MRI interpretations of independent readers with pathologic findings, including seminal vesicle invasion (SVI) and the presence of ECE overall and in the area of the NVB.[9-14] To our knowledge, none of these studies has prospectively examined the impact of preoperative MRI on the surgical management decision to preserve or resect an NVB during RP. We undertook the current prospective study to determine the incremental value of preoperative eMRI on the decision to preserve or resect NVBs during RP, using step-section histopathologic findings as the standard of reference.

\section{Materials and Methods}

\section{Study Population}

This prospective, single-institution, cross-sectional study was conducted between March 2001 and February 2003. A total of 144 consecutive patients with biopsy-proven prostate carcinoma who were scheduled to undergo RP based on clinical assessment of the stage of the tumor were referred for eMRI by one of two attending urologists (P.T.S. and J.E.). All patients were scheduled to undergo RP before referral for MRI. Scheduling for MRI depended on the MRI schedule as well as the availability of the surgeon and radiologist because a joint prospective reading was required prior to surgery. Patients who had previously undergone hormonal therapy, radiation therapy, or chemotherapy were excluded from the final analysis. Patients were recruited as part of an ongoing National Institutes of Health-funded study of MRI for prostate carcinoma. Our institutional review board approved the study and informed consent was obtained from the patients. All patients underwent standard preoperative evaluation for prostate carcinoma, including history, physical examination, digital rectal examination (DRE), measurement of the serum PSA level, and sextant biopsy. Clinical stage was assigned using the 1992 International Union Against Cancer (UICC) TNM staging system.[15]

Of the 144 patients referred for eMRI, 135 fulfilled the study inclusion criteria. Nine patients were excluded from the final analysis because of presurgical hormonal treatment (six patients), radiation therapy (one patient), or combined chemotherapy and radiation therapy (two patients). Table 1 lists the clinical findings of the 135 patients included in the data analysis. The median age of the patients was 58 years (range, 40-73 years). The median PSA level was 5.78 $\mathrm{ng} / \mathrm{mL}$ (mean, $6.17 \mathrm{ng} / \mathrm{mL}$; range, 1.06-76.84 $\mathrm{ng} / \mathrm{mL}$ ). Sextant biopsy of the prostate was performed at the study institution ( $n=28$ patients) or at an outside institution ( $n=107$ patients). A single pathologist at the study institution center reviewed all biopsy specimens. The median interval from biopsy to MRI was 8 weeks (range, 1.4-84 weeks). The median interval between MRI and radical prostatectomy was 6.3 weeks (range, $0.1-29$ weeks). 


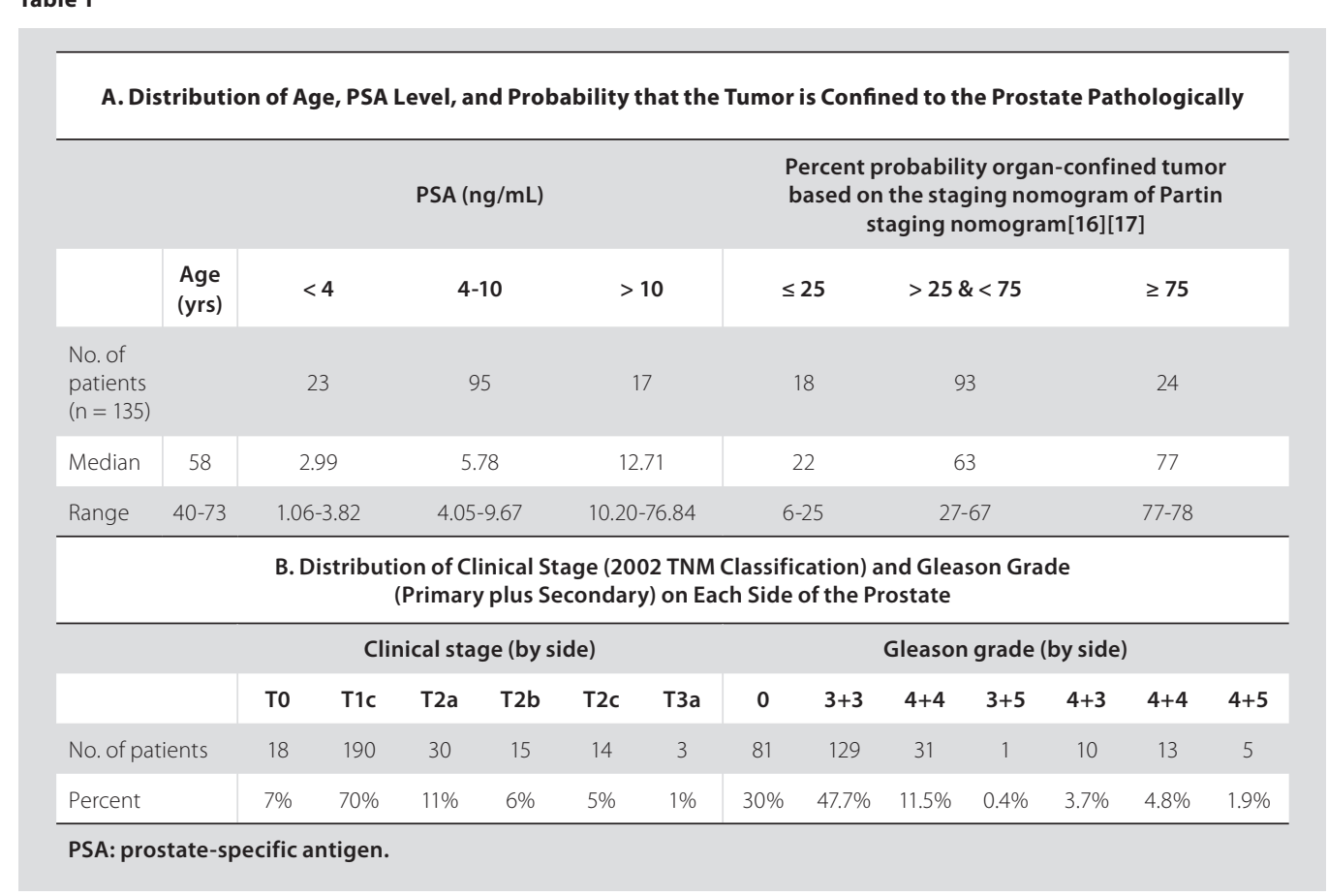

\section{MRI Technique}

MRI was performed on a 1.5-Tesla whole body MR scanner (Signa ${ }^{\oplus}$; GE Medical Systems, Milwaukee, WI). Patients were examined in the supine position, using the body coil for excitation and a pelvic-phased array coil (GE Medical Systems) in combination with a commercially available balloon-covered expandable endorectal coil (Medrad Inc., Pittsburgh, PA) for signal reception. T1-weighted, axial spin-echo images (Repetition Time/Echo Time [TR/TE] = 700/8 milliseconds [msec], slice thickness of 5 mm, interslice gap of $1 \mathrm{~mm}$, field of view [FOV] of $24 \mathrm{~cm}$, matrix of 256 ?192, frequency direction transverse, 1 excitation) were obtained from the aortic bifurcation to the symphysis pubis, and T2-weighted axial and coronal fast spin-echo images (TR/effective/TE $=5000 / 96 \mathrm{msec}$, echo train length of 16, slice thickness of $3 \mathrm{~mm}$, interslice gap of $0 \mathrm{~mm}$, FOV of $14 \mathrm{~cm}$, matrix of 256 ?192, frequency direction anteroposterior, 3 excitations) were obtained through the prostate and seminal vesicles. All MR images of each patient in the current study were reviewed by one of two independent readers (H.H. and F.V.C.).

\section{MRI Interpretation}

MRI interpretation included analysis of tumor location and evaluation of tumor ECE in the region of the NVB (posterolateral). In addition, the image was evaluated for the presence of any ECE, SVI, or lymph node metastasis. Tumor was identified on T2-weighted imaging when there was a nodular, mass-like area of low signal intensity. The findings used to diagnose ECE on eMRI have been described previously and include: 1) an irregular bulge of the gland margin, 2) a contour deformity with a stepoff or angulated margin, 3) a breech of the capsule with direct tumor extension, 4) the obliteration of the rectoprostatic angle, and 5) asymmetry and/or tumor envelopment of the NVBs.[10-14] Both readers used the same MR criteria. The MRI likelihood for ECE was scored on a scale of 1 to 5 as follows: 1: no ECE; 2: probably no ECE; 3: possible ECE; 4: probable ECE; and 5: definite ECE. Only a single reading was performed for each patient. The choice of the MRI reader was determined by the availability of the radiologist at the time of interpretation. Although the readers differed with regard to years of experience, both had read $>500$ eMRIs of the prostate.

\section{Determination of Pre-MRI and Post-MRI Surgical Plans}

A data sheet was prepared for each patient by one of two independent coauthors (L.W. and D.C.W.) not involved in the image interpretation or clinical decision making. The data presented were DRE findings, serum PSA level, and sextant biopsy results (including Gleason primary and secondary grade, tumor location, percentage of positive bi- 
opsy cores, and the percentage of tumor-involved core tissue). Published Partin tables (2001 version)[16] were used to estimate (based on the preoperative stage, Gleason grade, and PSA level) the probability that the tumor was histopathologically confined to the prostate gland ( organ-confined ).[16][17] Using all available clinical information, the attending urologist formulated a surgical plan for each NVB on a scale from 1 (definite preservation) to 5 (definite resection), designated the pre-MRI plan. The attending urologist then reviewed the MR images in consultation with an attending radiologist and formulated the post-MRI surgical plan on the same 5 -point scale. Therefore, the post-MRI plan was based on a combination of the clinical and MRI findings. Intraoperative management was guided by the post-MRI plan, occasionally modified by intraoperative findings.

\section{RP and Histologic Evaluation}

RP was performed as previously described,[18] with nerve-sparing ( $n=223$ patients), partial nerve resection ( $n=7$ patients), or complete nerve resection ( $n=40$ patients). Once the prostate was removed, the specimen was coated with India ink (the right and left sides were inked in 2 colors) and fixed in 10\% buffered formaldehyde.[19] The apical prostate was truncated and axial step-sections were obtained at 3-4-mm intervals in a plane perpendicular to the long axis of the prostate. The presence of tumor cells beyond the capsular margin defined ECE.

\section{Standard of Reference}

The standard of reference for the incremental value of MRI was based on histopathologic findings from the surgical specimen. Regardless of the actual surgery performed, the optimal (appropriate) surgical plan was determined based on histopathologic findings of ECE or positive surgical margins in the region of the NVB, posterolateral to the prostate. For each NVB, preservation was considered appropriate if the tumor did not extend outside the capsule in the posterolateral region of the prostate and the adjacent surgical margin was negative. NVB resection was considered appropriate if there was ECE or if there was a positive surgical margin in the posterolateral region of the prostate.

\section{Statistical Analysis}

A total of 270 NVBs were evaluated in 135 patients. The effect of eMRI on NVB management was examined for the frequency and the appropriateness of change between the pre-MRI and post-MRI surgical plans. The surgical plan was recorded as more conservative or more aggressive according to whether it included NVB preservation or NVB resection, respectively. To compare the accuracy of the surgeon's pre-MRI and post-MRI judgments, we used a receiver operating characteristic (ROC) curve approach. Specifically, we estimated the parameters of the ROC curves using a latent variable binormal model. The model included a random effect to account for the two observations per patient, right and left NVBs, both pre-MRI and post-MRI. Model estimates were obtained using maximum likelihood and the accuracy of pre-MRI and post-MRI were compared using a likelihood ratio test.[20]

To investigate whether MRI provided a benefit for both high-risk and low-risk patients, the Partin nomogram score was used as a continuous variable and the nomogram-MRI interaction was used as covariate in the ordinal regression model. To better visualize the results of the risk-group analysis we created low-risk and high-risk groups. If a patient's chance of having organ-confined disease was $>75 \%$ as predicted by the published tables,[16] he was classified as being at low risk; if the patient had a $<75 \%$ chance of having organ-confined disease, he was classified as high risk. To assess the management change, we furthermore dichotomized the pre-MRI and post-MRI ratings as negative $(1,2$, or 3 ) or positive ( 4 or 5$)$.

Because Partin staging nomograms are not universally used, we performed an additional analysis using a simplified, clinically defined, low-risk group (PSA level < $10 \mathrm{ng} / \mathrm{ML}$, Gleason grade of 6, and clinical T1c or T2a disease). Furthermore, because the percentage of positive biopsy cores is often used as an indicator for NVB resection, correlation of the percentage of positive biopsy cores with the incremental value of MRI was performed as well. 


\section{RESULTS}

Based on the histopathologic findings, NVB resection was considered the appropriate surgery for 44 of 270 NVB sides (16\%) because of posterolateral ECE $(n=29)$, positive surgical margins $(n=7)$, or both $(n=8)$. Overall, 32 patients had ECE; in 7 of these patients (22\%) the ECE was located only anteriorly or anterolaterally and away from the NVB and therefore did not warrant NVB resection.

The post-MRI surgical plan, including the decision to preserve or resect the NVB, agreed with the pre-MRI clinical surgical plan for 164 of 270 NVBs (61\%). The surgeon judged that NVB resection was definitely not necessary in 165 NVBs and the eMRI confirmed that decision in 138 NVBs (84\%). Of the 138 NVB sites in which the clinical decision concurred with the post-MRI decision not to resect an NVB, the decisions were correct for 133 of the sites (96\%). MRI findings suggested a change in the surgical plan for 106 of 270 NVBs (39\%). Thirty-nine NVBs had a more conservative surgical plan post-MRI; in 35 cases (90\%), this change was appropriate. Overall, 67 NVBs had a more aggressive surgical plan post-MRI; in 45 cases (67\%), this change was appropriate. Table 2 compares the pre-MRI and post-MRI surgical plans and shows the influence of MRI findings and its appropriateness. Two representative cases are illustrated in Figures 1 and 2. Table 3 lists the incremental effect of MRI stratified by the likelihood of extraprostatic disease from published staging tables.[16][17] For patients with a high probability of extraprostatic disease (probability of organ-confined disease of 25\%), MRI changed the NVB management in 28 of 36 sites (78\%); in 26 of these 28 sites (93\%), the change was appropriate. In one patient, MRI suggested unilateral ECE with the involvement of $N V B$, prompting the more aggressive post-MRI surgical plan. At the time of surgery, no ECE was found in this patient, although there was extensive neural invasion within the prostate and a moderate-volume tumor (Fig. 3).

Table 2. Pre-MRI and Post-MRI Surgical Plans According to the Surgeon's Intended Probability of Preserving (1) or Resecting (5) the Ipsilateral NVB

\begin{tabular}{|c|c|c|c|c|c|c|c|c|}
\hline \multirow[b]{3}{*}{ NVB } & \multirow{2}{*}{\multicolumn{2}{|c|}{ Pre-MRI plan }} & \multicolumn{6}{|c|}{ Post-MRI plan ${ }^{a}$} \\
\hline & & & \multicolumn{2}{|c|}{ No change } & \multicolumn{2}{|c|}{ More conservative } & \multicolumn{2}{|c|}{ More aggressive } \\
\hline & Score & No. of NVB & No. of NVB & Appropriate & No. of NVB & Appropriate & No. of NVB & Appropriate \\
\hline \multicolumn{9}{|c|}{ Preserve } \\
\hline & 1 & 165 & 138 & 133 & NA & NA & 27 & 22 \\
\hline & 2 & 55 & 16 & 14 & 19 & 17 & 20 & 3 \\
\hline & 3 & 25 & 7 & 7 & 11 & 10 & 7 & 7 \\
\hline & 4 & 23 & 1 & 1 & 9 & 8 & 13 & 13 \\
\hline \multirow[t]{2}{*}{ Resect } & 5 & 2 & $2^{b}$ & 2 & 0 & 0 & 0 & 0 \\
\hline & & 270 & 164 & $157(96 \%)$ & 39 & $35(90 \%)$ & 67 & $45(67 \%)$ \\
\hline
\end{tabular}

MRI: magnetic resonance imaging; NVB: neurovascular bundle; NA: not applicable.

a Postmagnetic resonance imaging surgical plans were stratified as no change, more conservative, or more aggressive surgical approaches.

b When the surgeon judged that neurovascular bundle resection was definitely necessary (in 2 neurovascular bundles), the postmagnetic resonance imaging plan confirmed that decision as appropriate in 2 neurovascular bundles (100\%) 


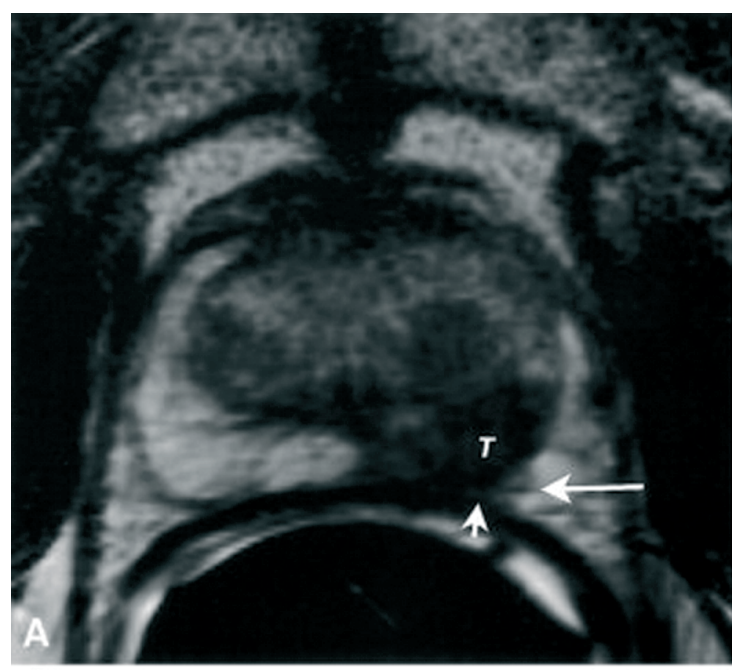

Figure 1. Case 1. Clinical stage T2a prostate carcinoma with a Gleason score of 7 in a 61-year-old patient with a prostate-specific antigen (PSA) level of $5.4 \mathrm{ng} / \mathrm{mL}$. The premagnetic resonance imaging (preMRI) surgical plan indicated probable preservation of the left neurovascular bundle (NVB) (a score of 2 of 5, where 1 indicated definite preservation of the NVB and 5 indicated definite resection of the NVB) and probable resection of the right NVB (a score of 4 of 5). (A) An axial T2-weighted MRI image showed a large low-signal intensity tumor (T) in the left peripheral zone; there was obliteration of the rectoprostatic angle (arrowhead) and asymmetry of the NVB (arrow); both findings indicate extracapsular extension (ECE) (MRI score of 5). No tumor was noted in the right peripheral zone. As a result, the surgical plan was changed and was more aggressive on the left NVB (changing from 2 to 5) and less aggressive on the right NVB (changing from 4 to 1). (B) A corresponding histopathologic step-section map demonstrated established ECE at the left posterior (base); all surgical margins were free of tumor. MRI findings appropriately changed the clinical impression of the urologist and the surgical plan for the left and right NVB. (Color version available in chapter 18).

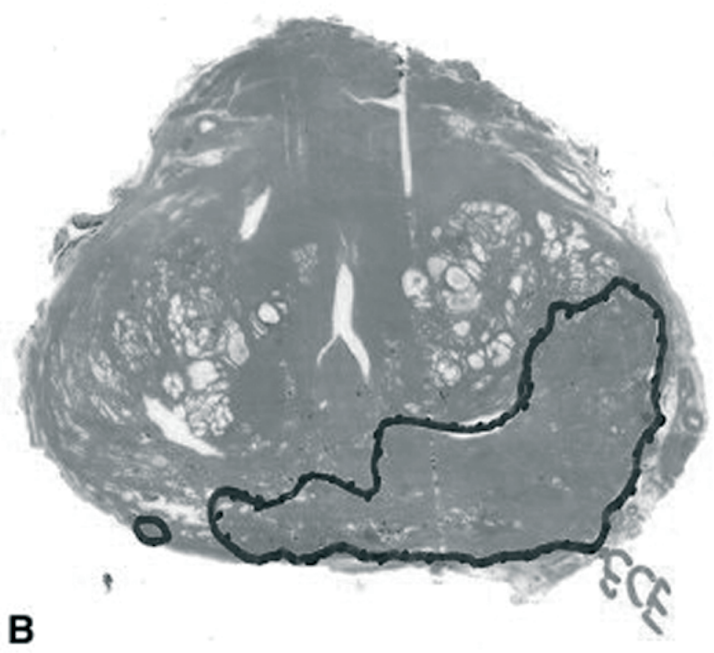

Table 3. Appropriateness of Changes in the Post-MRI Surgical Plans Grouped According to the Preoperative Probability that the Tumor Was Confined to the Prostate Pathologically[16][17]

\begin{tabular}{|c|c|c|c|c|c|c|c|c|}
\hline \multicolumn{2}{|c|}{$\begin{array}{c}\text { Percent } \\
\text { probability } \\
\text { of organ-confined } \\
\text { tumor }\end{array}$} & \multicolumn{2}{|c|}{$\begin{array}{l}\text { No change post- } \\
\text { MRI plan }\end{array}$} & \multicolumn{2}{|c|}{$\begin{array}{c}\text { More } \\
\text { conservative } \\
\text { post-MRI plan }\end{array}$} & \multicolumn{2}{|c|}{$\begin{array}{c}\text { More } \\
\text { aggressive } \\
\text { post-MRI plan }\end{array}$} & \multirow{2}{*}{$\begin{array}{c}\begin{array}{c}\text { Appropriate } \\
\text { post-MRI } \\
\text { altered } \\
\text { surgical } \\
\text { plans }\end{array} \\
\text { No. of NVB (\%) }\end{array}$} \\
\hline Probability & No. of NVB & No. of NVB & Appropriate & No. of NVB & Appropriate & No. of NV & Appropriate & \\
\hline$>75 \%$ & 47 & 38 & 37 & 0 & 0 & 9 & 6 & 6/9 (67\%) \\
\hline $51-74 \%$ & 127 & 91 & 86 & 11 & 9 & 25 & 13 & 22/36 (61\%) \\
\hline $26-50 \%$ & 60 & 27 & 26 & 16 & 16 & 17 & 10 & 26/32 (81\%) \\
\hline$<25 \%$ & 36 & 8 & 8 & 12 & 10 & 16 & 16 & 26/28 (93\%) \\
\hline Totals & 266 & 162 & 155 & 39 & 35 & 65 & 45 & $80 / 104(77 \%)$ \\
\hline
\end{tabular}



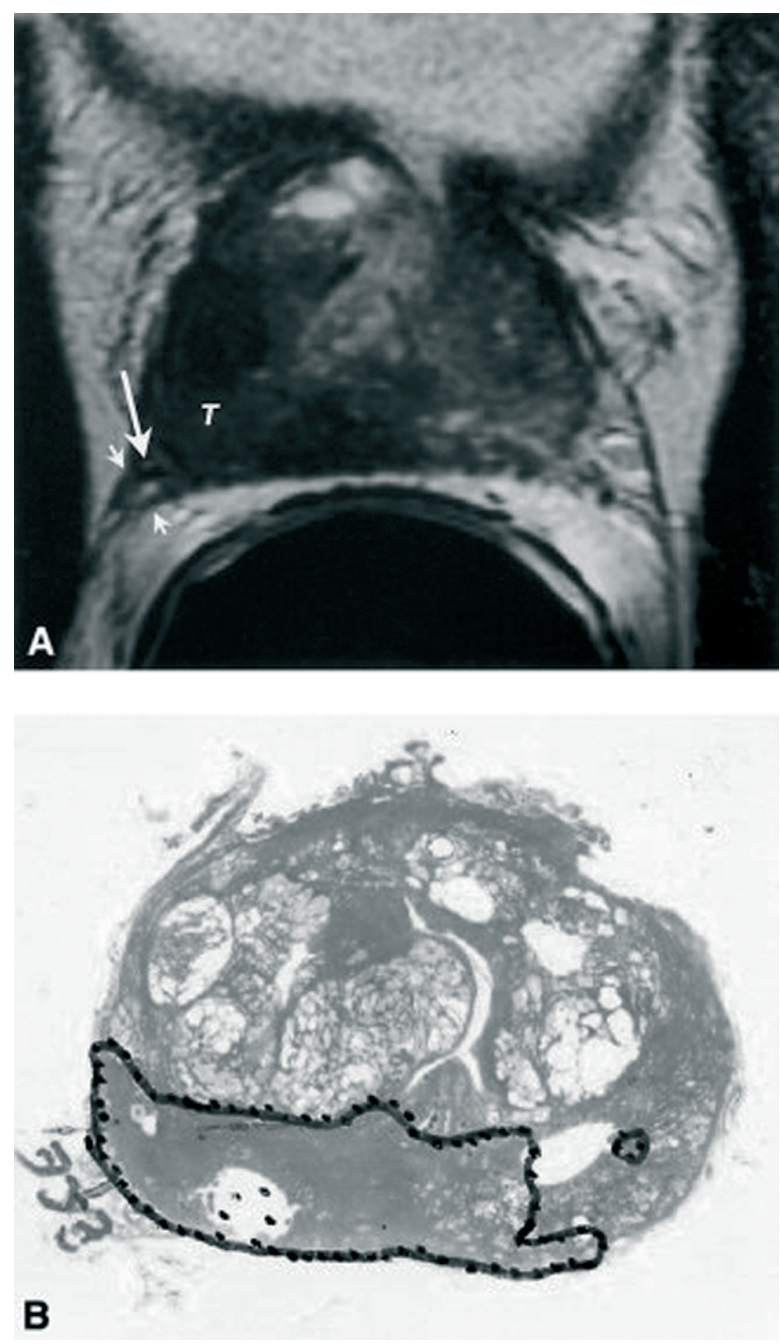

Figure 2. Case 2. Clinical stage T1c prostate carcinoma with a Gleason score of 9 in a 60-year-old patient with a prostate-specific antigen (PSA) level of $8.45 \mathrm{ng} / \mathrm{mL}$. The premagnetic resonance imaging (pre-MRI) surgical plan indicated probable preservation of the right neurovascular bundle (NVB) (a score of 2 of 5, where 1 indicated definite preservation of the NVB and 5 indicated definite resection of the NVB) and possible resection of the left NVB (a score of 3 of 5). (A) An axial T2-weighted MRI image showed a large low-signal intensity tumor $(\mathrm{T})$ in the right peripheral zone. The contour of the gland was angulated and there was direct tumor extracapsular extension (ECE) (arrowheads), and envelopment of the right NVB (arrow); findings indicated right-sided ECE (MRI score of 5). There was no MRI evidence of left-sided ECE. As a result, the surgical plan was altered and was more aggressive with regard to the right NVB (changing from 2 to 5). The surgical plan for the left NVB remained unchanged. (B) A corresponding histopathologic step-section map showed established, posterior, right-sided ECE. In this case, aggressive management of the right NVB was appropriate.

(Color version available in chapter 18).

ROC curves for pre-MRI and post-MRI surgical plans are plotted in Figure 4. The area under the curve (AUC) was 0.741 for pre-MRI and 0.832 for post-MRI. The difference between the two curves was found to be statistically significant $(P<0.01)$, suggesting that MRI substantially improves the accuracy of the surgeon's judgment with regard to the appropriateness of preserving or resecting NVBs.

Table 4. Changes in the Post-MRI Surgical Plans Grouped According to the Preoperative Probability that the Tumor was Confined to the Prostate Pathologically as Defined by a PSA Level $<10 \mathrm{ng} / \mathrm{mL}$, a Gleason Score $<6$, and T1c or T2a Disease

\begin{tabular}{lcc} 
Risk category & Pre-MRI AUC & Post-MRI AUC \\
\hline Low & 0.647 & 0.647 \\
High & 0.783 & 0.983 \\
All & 0.741 & 0.832
\end{tabular}

MRI: magnetic resonance imaging; PSA: prostate-specific antigen; AUC: area under the curve. 


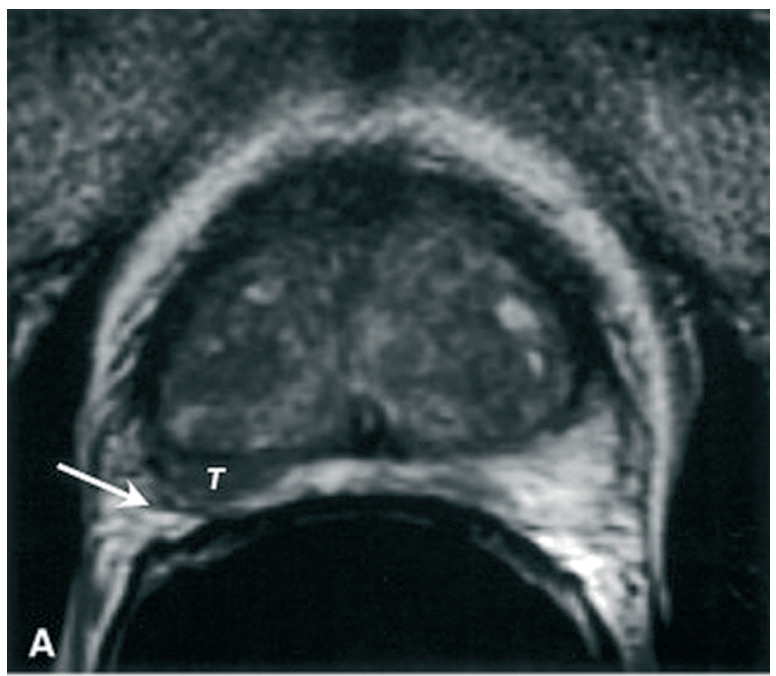

Figure 3. Case 3. Clinical stage T1c prostate carcinoma with a Gleason score of 6 in a 55-year-old patient with a prostate-specific antigen (PSA) level of $6.31 \mathrm{ng} / \mathrm{mL}$. The promagnetic resonance imaging (pre-MRI) surgical plan indicated definite preservation of both neurovascular bundles (NVBs) (a score of 1 of 5 for both, where 1 indicated definite preservation of the NVB and 5 indicated definite resection of the NVB). (A) An axial T2-weighted MRI image demonstrated a focal low-signal intensity tumor (T) in the right peripheral zone. The tumor extended to the capsular margin in the region of the right NVB (arrow). Because the tumor abutted the right NVB, early extracapsular extension (ECE) could not be excluded (MRI score of 3). Consequently, the surgical plan was changed and was more aggressive with regard to the right NVB (changing from 1 to 3). MRI showed no tumor on the left. Thus, the surgical plan for the left NVB remained unchanged. (B) A corresponding histopathologic step-section map revealed no ECE and all surgical margins were free of tumor. In this case, MRI findings inappropriately changed the surgical plan of the urologist with regard to the right NVB but confirmed the decision to preserve the left NVB.

(Color version available in chapter 18).

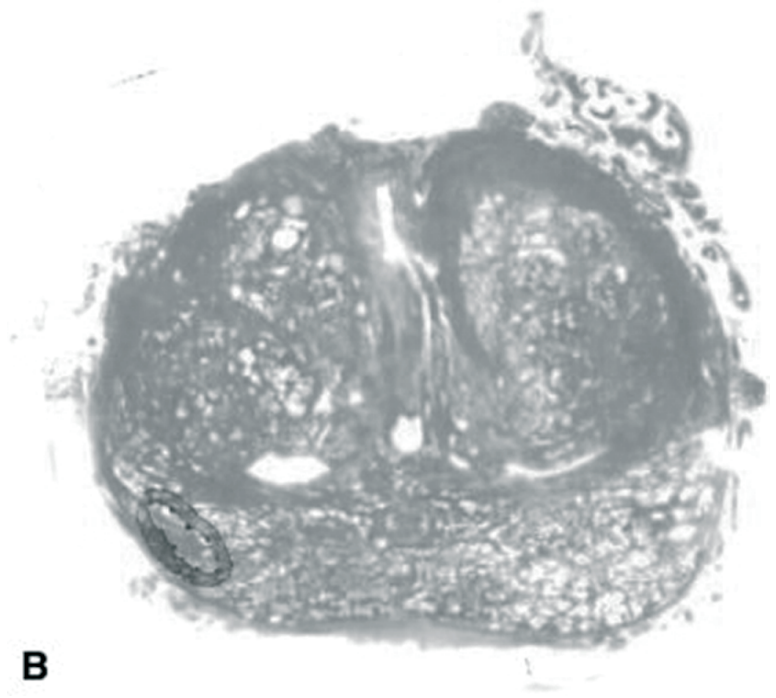

In the ordinal regression model, MRI retained its significance $(P<0.01)$ and the nomogram also was found to be significant $(P<0.01)$. The nomogram-MRI interaction had a negative sign, suggesting that the benefit of MRI dissipates as the probability of having organ-confined disease increases, implying less benefit for low-risk patients, although this finding did not reach statistical significance $(P=0.08)$.

The analysis using a clinically defined low-risk group (PSA level $<10 \mathrm{ng} / \mathrm{mL}$, a Gleason grade of 6 , and clinical stage T1c or T2a disease) confirmed the Partin staging nomogram analysis.[16][17] and showed that, for low-risk patients, the incremental value of MRI in the decision to change the surgical approach is minimal (Table 4). Similarly, when the data were dichotomized for the percentage of positive biopsy cores at 25\% (observed median), MRI was found to have a greater incremental value for the high-risk group of patients (Table 5). 
Table 5. Appropriateness of Changes in the Post-MRI Surgical Plans Grouped According to the Preoperative Probability that the Tumor Was Confined to the Prostate Pathologically as Dichotomized at 25\% (Observed Median) for the Percentage of Positive Biopsy Cores

\begin{tabular}{lcc} 
Category & Pre-MRI AUC & Post-MRI AUC \\
\hline$<0.25$ & 0.736 & 0.695 \\
$>0.25$ & 0.742 & 0.925 \\
All & 0.741 & 0.832
\end{tabular}

MRI: magnetic resonance imaging; AUC: area under the curve.

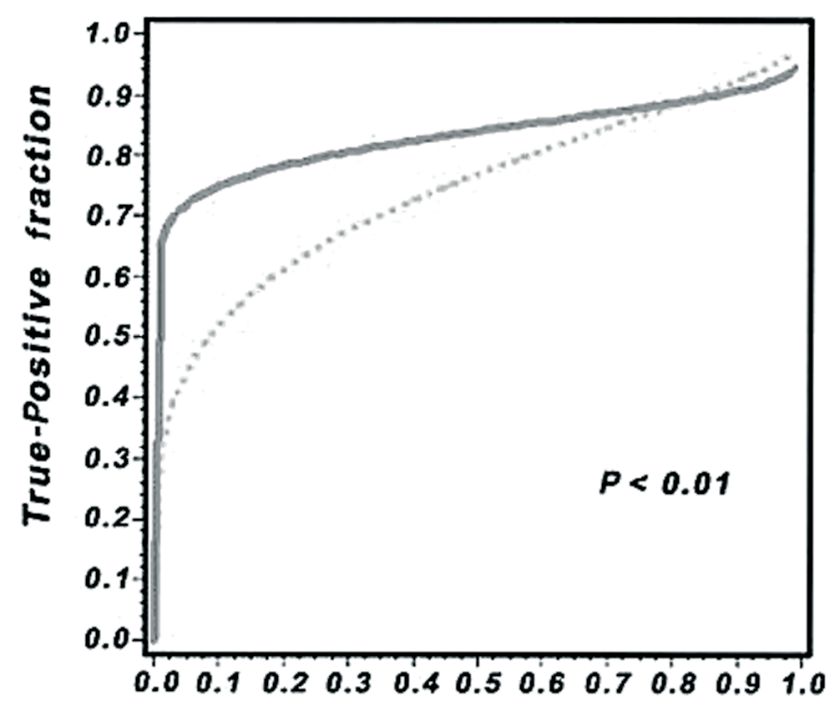

False-positive fraction
Figure 4. Graphic representation of the accuracy of the premagnetic resonance imaging (pre-MRI) and postmagnetic resonance imaging (post-MRI) surgical plans using receiver operating characteristic $(\mathrm{ROC})$ analysis. Solid line: pre-MRI (area under the curve $=0.741$ ); dotted line: post-MRI (area under the curve $=0.832$ ).

Analysis of the management change (no resection [score of 1-3] to resection [score of 4-5]) in the post-MRI surgical plan is presented in Table 6. The management change was initiated in $32 \mathrm{NVBs}$, and was found to be appropriate in 29. Although the overall impact of MRI was found to be significant, the benefit was greatest in high-risk patients. In 24 high-risk patients, the pre-MRI and post-MRI plans disagreed; in 22 of these patients, the post-MRI classification was correct. These results indicate that adding MRI was useful for patients who had a low probability of organ-confined disease. 
Table 6. Summary of the Impact of MRI on Surgical Management

\begin{tabular}{|c|c|c|c|c|}
\hline & Pre-MRI plan & Post-MRI plan & No. of NVBs & Appropriate \\
\hline \multirow[t]{3}{*}{ MRI affected surgical management } & $1,2,3$ & 4,5 & 15 & $14(93 \%)$ \\
\hline & $3,4,5$ & 1,2 & 17 & $15(88 \%)$ \\
\hline & \multicolumn{2}{|c|}{ Overall } & 32 & $29(91 \%)$ \\
\hline \multirow[t]{6}{*}{ MRI change in score did not affect management } & 1 & 2 & 18 & \\
\hline & 2 & 1 & 19 & \\
\hline & 4 & 5 & 13 & \\
\hline & 5 & 4 & 0 & \\
\hline & 1,2 & 3 & 21 & \\
\hline & 4,5 & 3 & 3 & \\
\hline
\end{tabular}

MRI: magnetic resonance imaging; NVBS: neurovascular bundles.

\section{Discussion}

The role of eMRI in the staging of and surgical planning for prostate carcinoma continues to provoke controversy.[18][21] A large multiinstitutional trial published in 1990 suggested that the accuracy of MRI for detecting ECE or SVI was low.[22] However, in the last decade MRI has improved considerably with technologic refinements and increased reader experience.[9] Among other technologic improvements, and pertinent to the evaluation of the prostate gland, was the introduction of eMRI. eMRI technology provides a more detailed anatomic image of the prostate compared with body coil MRI technology.[10][23] The MRI data obtained in the late 1980s used MRI technology that is now obsolete; therefore, the data are not representative of current state-of-the-art imaging capabilities. Furthermore, the 1990 multicenter study as well as most of the subsequent MRI studies focused on imaging pathologic correlation and MRI technology assessment.[9-11][21] We are unaware of any studies investigating the effect of preoperative eMRI on surgical management. However, the prognostic ability of preoperative MRI has been reported recently.[21][23-27] In a retrospective study of 1025 patients undergoing RP, T-stage classification by MRI was found to be an additional independent predictor of postoperative PSA failure $(P<0.001)$ for patients at intermediate and high risk.[24] Although the study had a different endpoint from the current report, it is interesting to note that both studies suggest that MRI is more helpful in patients with unfavorable prognostic features.

The results of the current study suggest two important management benefits of eMRI prior to RP: improved surgical planning in high-risk patients and the provision of appropriate reassurance for preserving NVB in other patients. In the current study, we used the 2001 version of the Partin staging tables[16][17] for risk stratification because it is a validated predictive instrument that is widely used for patient counseling.[28][29] Patients with a probability of organ-confined tumor of $25 \%$ were considered to be high risk in the current study. MRI findings changed the surgical plan in $78 \%$ of these high-risk patients; in $93 \%$ of these cases, the changes were appropriate. Despite the high clinical probability of extraprostatic disease in these patients, MRI correctly favored NVB preservation in 10 of 12 NVBs (83\%). In low-risk and intermediate-risk groups, MRI helped to provide appropriate reassurance in those cases in which NVB preservation was favored on the pre-MRI surgical plan. Overall, the strength of MRI for low-risk and intermediate-risk patients lies in a high negative predictive value (i.e., demonstration of the absence of tumor in the region of interest). In the high-risk group, MRI has incremental value in the clinical assessment additive to the Partin staging nomogram[16][17] a simplified clinical risk stratification (using PSA level, Gleason grade, and clinical disease stage), or the percentage of positive biopsy cores.

\section{Limitations}

The current study has several limitations. First, the study design and results are based on personal consultation between experienced urologists and radiologists, all of whom were aware of the clinical findings, such as the location and percentage of positive biopsy cores and the percentage of tumor-involved core tissue. This multidisciplinary 
approach should optimize the interpretation of MRI findings and allow an optimal assessment of the incremental effect of MRI; however, it might not be representative of widespread daily clinical practice. Second, reflective of current surgical practice, a high percentage of patients had favorable pathologic results. With such a small prior probability, and therefore a small number of patients who required NVB resection, statistical validation of incremental change was difficult to ascertain and will require further analysis.

\section{SUMmARY}

eMRI prior to RP was found to improve the validity of the surgical decision to preserve or resect the NVB. Thus, preoperative eMRI can provide additional, critical information that allows treatment to be adjusted to an optimal, patient-specific therapy.

\section{References}

1 Hull GW, Rabbani F, Abbas F, Wheeler TM, Kattan MW, Scardino PT. Cancer control with radical prostatectomy alone in 1,000 consecutive patients. J Urol. 2002; 167(2 Pt 1): 528-534.

2 Rabbani F, Stapleton AM, Kattan MW, Wheeler TM, Scardino PT. Factors predicting recovery of erections after radical prostatectomy. $J$ Urol. 2000; 164: 1929-1934.

3 MCNeal JE. Cancer volume and site of origin of adenocarcinoma in the prostate: relationship to local and distant spread. Hum Pathol. 1992; 23(3): 258-266.

4 Eastham JA, Scardino PT. Early diagnosis and treatment of prostate cancer. Dis Mon. 2001; 47(9): 421-459.

5 Rosen MA, Goldstone L, Lapin S, Wheeler T, Scardino PT. Frequency and location of extracapsular extension and positive surgical margins in radical prostatectomy specimens. J Urol. 1992; 148(2Pt 1): 331-337.

6 Kim ED, Nath R, Kadmon D, et al. Bilateral nerve graft during radical retropubic prostatectomy: 1-year followup. J Urol. 2001; 165(6 Pt 1): 1950-1956.

7 Sofer M, Hamilton-Nelson KL, Schlesselman JJ, Soloway MS. Risk of positive margins and biochemical recurrence in relation to nervesparing radical prostatectomy. J Clin Oncol. 2002; 20 (7): 1853-1858.

8 D'Amico AV, Whittington R, Malkowicz SB, et al. Combined modality staging of prostate carcinoma and its utility in predicting pathologic stage and postoperative prostate specific antigen failure. Urology. 1997; 49(3A Suppl): 23-30.

9 D'Amico AV, Whittington R, Malkowicz SBE, et al. Critical analysis of the ability of the endorectal coil magnetic resonance imaging scan to predict pathologic stage, margin status, and postoperative prostate-specific antigen failure in patients with clinically organ-confined prostate cancer. J Clin Oncol. 1996; 14: 1770-1777.

10 Yu KK, Hricak H, Alagappan R, Chernoff DM, Bacchetti P, Zaloudek CJ. Detection of extracapsular extension of prostate carcinoma with endorectal and phased-array coil MR imaging: multivariate feature analysis. Radiology. 1997; 202: 697-702.

11 Ikonen S, Karkkainen P, Kivisaari L, et al. Magnetic resonance imaging of clinically localized prostatic cancer. J Urol. 1998; 159: 915-919.

12 Yu KK, Scheidler J, Hricak H, et al. Prostate cancer: prediction of extracapsular extension with endorectal MR imaging and threedimensional proton MR spectroscopic imaging. Radiology. 1999; 213: 481-488.

13 Ogura K, Maekawa S, Okubo K, et al. Dynamic endorectal magnetic resonance imaging for local staging and detection of neurovascular bundle involvement of prostate cancer: correlation with histopathologic results. Urology. 2001; 57(4): 721-726.

14 Tempany CM, Rahmouni AD, Epstein JI, Walsh PC, Zerhouni EA. Invasion of the neurovascular bundle by prostate cancer: evaluation with MR imaging. Radiology. 1991; 181: 107-112.

15 Han M, Walsh PC, Partin AW, Rodriguez R. Ability of the 1992 and 1997 American Joint Committee on Cancer staging systems for prostate cancer to predict progression-free survival after radical prostatectomy for stage T2 disease. J Urol. 2000; 164: 89-92.

16 Partin AW, Mangold LA, Lamm DM, Walsh PC, Epstein JI, Pearson JD. Contemporary update of prostate cancer staging nomograms (Partin Tables) for the new millennium. Urology. 2001; 58: 843-848.

17 Partin AW, Kattan MW, Subong EN, et al. Combination of prostate-specific antigen, clinical stage, and Gleason score to predict pathological stage of localized prostate cancer. A multi-institutional update. JAMA. 1997; 277: 1445-1451.

18 Eastham JA, Scardino PT: Radical prostatectomy for clinical stage T1 and T2 prostate cancer. In: Vogelzang NJ , Scardino PT, Shipley WU , Coffey DS, editors. Comprehensive textbook of genitourinary oncology. 2nd edition. Philadelphia: Lippincott Williams and Wilkins, 2000: 722.

19 Yossepowitch O, Engelstein D, Konichezky M, Sella A, Livne PM, Baniel J. Bladder neck involvement at radical prostatectomy: positive margins or advanced T4 disease. Urology. 2000; 56(3): 448-452.

20 McCulloch CE, Searle SR. Generalized, linear, and mixed models. New York: John Wiley \& Sons, 2001.

21 Coakley FV, Kurhanewicz J, Lu Y, et al. Prostate cancer tumor volume: measurement by endorectal MR imaging and MR spectroscopic imaging. Radiology. 2002; 223(1): 91-97.

22 Rifkin MD, Zerhouni EA, Gatsonis CA, et al. Comparison of magnetic resonance imaging and ultrasonography in staging early prostate cancer. Results of a multi-institutional cooperative trial. N Engl J Med. 1990; 323(10): 621-626.

23 Scheidler J, Hricak H, Vigneron DB, et al. Prostate cancer: localization with three-dimensional proton MR spectroscopic imaging clinicopathologic study. Radiology. 1999; 213: 473-480.

24 D'Amico AV, Whittington R, Malkowicz B, et al. Endorectal magnetic resonance imaging as a predictor of biochemical outcome after radical prostatectomy in men with clinically localized prostate cancer. J Urol. 2000; 164: 759-763.

25 Cornud F, Flam T, Chauveinc L, et al. Extraprostatic spread of clinically localized prostate cancer: factors predictive of pT3 tumor and of positive endorectal MR imaging examination results. Radiology. 2002; 224(1): 203-210.

26 Tempany CM, Zhou X, Zerhouni EA, et al. Staging of prostate cancer: results of Radiology Diagnostic Oncology Group project comparison of three MR imaging techniques. Radiology. 1994; 192: 47-54. 
27 Wefer AE, Hricak H, Vigneron DB, et al. Sextant localization of prostate cancer: comparison of sextant biopsy, magnetic resonance imaging and magnetic resonance spectroscopic imaging with step-section histology. J Urol. 2000; 164: 400-404.

28 Kattan MW, Stapleton AM, Wheeler TM, Scardino PT. Evaluation of a nomogram used to predict the pathologic stage of clinically localized prostate carcinoma. Cancer. 1997; 79: 528-537.

29 Blute ML, Bergstralh EJ, Partin AW, et al. Validation of Partin tables for predicting pathological stage of clinically localized prostate cancer. J Urol. 2000; 164: 1591-1595. 


\section{The Role of MRI in Improving Pre-Operative Nomograms to Predict Biochemical Recurrence-Free Probability in Patients Undergoing Radical Prostatectomy}

Supported by National Institutes of Health grant R01 CA76423

John F. Donohue, Liang Wang, Hedvig Hricak, Hui-Ni Chen, James A. Eastham, Peter T. Scardino, Michael W. Kattan

Departments of Urology and Radiology, Memorial Sloan-Kettering Cancer Center, Department of Quantitative Health Sciences, Cleveland Clinic Foundation

\section{Abstract}

Purpose: We investigated whether the addition of MRI findings to the pre-operative nomogram for predicting freedom from recurrence of prostate cancer would improve its predictive accuracy. Due to time constraints, comparison to actual biochemical recurrence events was not feasible. Instead, comparison was performed against a post-operative nomogram that predicts freedom from biochemical recurrence based on pathologic variables.

Methods: This study involved 592 consecutive patients with biopsy-proven prostate cancer who underwent MRI before radical prostatectomy. Pre-operative MRI reports were retrospectively reviewed and scores were assigned for the probability of biochemical recurrence on an arbitrary 7-point scoring system (scores increased with predicted pathologic stage). The MRI scoring system was combined with the Kattan pre-operative nomogram and linear regression analysis was performed to evaluate the combined model against the Kattan post-operative nomogram in predicting 10-year freedom from biochemical recurrence.

Results: Median follow-up was 22 months. Fifty-two (9\%) patients experienced biochemical recurrence. The addition of MRI scores to the pre-operative nomogram significantly improved the R-square value of the pre-operative nomogram in the total study cohort (0.58 vs. $0.53 ; \mathrm{P}<0.01$ ) and in a subset of patients (PSA $\geq 10$, Gleason score $\geq 7$ on biopsy or clinical stage T2) at high risk for biochemical recurrence (0.61. vs. 0.58; $p<0.01$ ).

Conclusion: The addition of MRI findings, when converted into a scoring system, significantly improves the accuracy of the Kattan pre-operative nomogram in predicting postoperatively determined recurrence-free probabilities. Further work is required to give weighted values to the scoring system, and time is needed to allow comparison with actual events. 


\section{INTRODUCTION}

$\mathbf{O}$

ptimal management of prostate cancer remains elusive. From diagnosis through staging, treatment planning and follow-up, there is a need to maximize information. A patient newly diagnosed with prostate cancer faces a number of treatment options. Predictions of their respective outcomes based on known factors can help both clinician and patient in choosing between them. To aid in patient counselling, pre-operative nomograms have been developed that give a prediction of the final pathologic stage and an estimate of the chances of freedom from disease recurrence after radical prostatectomy (RP). Predictors have been limited to PSA, clinical stage, Gleason grade and quantitative assessment of cancer in core biopsies.

Endorectal magnetic resonance imaging has been reported to improve detection of extracapsular extension (ECE),[1] seminal vesicle invasion (SVI)[2] and lymph node metastasis (LNM)[3] compared to standard clinical variables alone. Although the National Comprehensive Cancer Network recommends MRI for patients with clinical stage T3 or T4 or a nomogram risk of LNM greater than $20 \%,[4]$ it has been shown that MRI can assist in treatment planning[5-7] and prediction of pathologic stage for all patients with clinically localized prostate cancer.[8] The purpose of our study was to test the hypothesis that the addition of MRI findings to an established preoperative nomogram would improve noninvasive prediction of freedom from biochemical recurrence.

The gold standard for the accuracy of MRI in predicting pathologic stage is the surgical specimen. Biochemical recurrence of cancer is time dependent, and maturation of a dataset is required for enough events to occur for valid statistical analysis. In the Johns Hopkins series of 2404 men treated with RP, with a mean follow-up of 6.3 years, the overall actuarial recurrence-free rates at 5, 10 and 15 years were $84 \%, 74 \%$ and $66 \%$, respectively.[9]

As MRI has only recently begun to be used more freely, it would take many years for our cohort of patients to develop enough recurrence events to demonstrate whether the addition of MRI to the pre-operative nomogram improves its ability to predict recurrence. Therefore, in the present study, the "gold standard" we used for comparison was the predicted probability of freedom from biochemical recurrence calculated from the post-operative nomogram, which, given its use of pathologic variables, is more accurate than the pre-operative nomogram.[10]

\section{Methods}

\section{Patient Selection}

This is a study of 592 consecutive patients with biopsy-proven prostate cancer who were referred for endorectal MRI prior to RP between January 2000 and October 2004. For all patients undergoing RP at our institution, clinical and pathologic data are prospectively entered into a computerized database. Institutional Review Board approval was granted for retrospective analysis of the data in this study. Clinical stage was assigned preoperatively according to the 2002 International Union Against Cancer TNM staging system. Patients who received neoadjuvant hormonal therapy, neoadjuvant chemotherapy, or prior radiation treatment to the pelvis were excluded from the analysis. Follow-up included measurement of serum PSA and digital rectal examination every 3 months for the first year after surgery, at 6-month intervals for the next 4 years, and annually thereafter. Cancer recurrence was defined as a serum PSA $\geq 0.2 \mathrm{ng} / \mathrm{ml}$ and rising, clinical local or distant disease, or secondary therapy commenced at least 3 months after RP.

\section{Needle biopsy specimens}

All prostate biopsies were obtained using transrectal ultrasound guidance and were reviewed by dedicated uropathologists. Each core containing cancer was assigned a primary and secondary Gleason grade. Patients referred from outside institutions had a review of their prostate biopsies and assignment of Gleason grade by an MSKCC pathologist. An overall Gleason score was given to each case by identifying the core with the highest Gleason score. The total number of cores obtained, the number of cores containing cancer, and the percentage of cancer in the cores were recorded.

\section{Radical prostatectomy specimens}

Whole-mount sections of prostate were prepared as previously described.[11] The location and extent of invasive tumors were identified and precisely mapped in each section. Overall Gleason score was defined by the Gleason 
score of the tumor with the highest Gleason score. Pathologic parameters recorded included the presence or absence of ECE, SVI, and LNM. The level of extension was recorded routinely as none, invasion into the capsule, focal extracapsular extension, or established extracapsular extension, with the latter two defining ECE. An organ-confined tumor was defined as a tumor with no evidence of ECE, SVI, or LNM. The presence of tumor cells at the inked resection level was considered a positive surgical margin.

\section{Endorectal MRI}

Endorectal MRI was performed on a 1.5 Tesla whole-body magnetic resonance scanner (Signa Horizon scanner, General Electric Medical Systems, Milwaukee, WI). Patients were examined using a previously described protocol.1 Pre-operative endorectal MRI reports from medical records were retrospectively reviewed by a radiologist (LW), who knew that all patients had been diagnosed with prostate cancer and treated with RP but was unaware of other clinical data or outcomes. An arbitrary 7-point scoring system was developed to predict the probability of biochemical recurrence based on the MRI reports. The higher the score, the greater the probability of recurrence predicted. A score was retrospectively assigned for each MR report. The 7-point scoring system based on the TNM system was 1. no tumor seen; 2. tumor seen, no ECE; 3. tumor seen, ECE cannot be ruled out; 4. unilateral ECE; 5. bilateral ECE; 6. SVI; 7. LNM. When multiple positive findings on MRI were seen, the highest applicable score was assigned.

\section{Nomogram predictions}

The pre-operative variables (PSA, clinical stage, biopsy Gleason score and biopsy core information) were used to determine the 10-year progression-free probabilities in each case using the Kattan pre-operative nomogram.[12] Similarly, post-operative variables (pre-treatment PSA, Gleason score, level of prostate capsular invasion, SVI, LNM and positive surgical margins) were used in the updated Kattan post-operative nomogram10 to calculate the 10year progression-free probabilities.

\section{Statistical Analysis}

Linear regression analysis was performed to assess the association between the predictors, the pre-operative nomogram with and without the addition of the MRI scores, and the post-operative nomogram. Restricted cubic spline function was used on the predictor pre-operative nomogram. In addition to the significance level of each variable, model r-squares were calculated. When the pre-operative nomogram was combined with the MRI scoring system in the model, bootstrapping with 200 resamples was used to calculate bias-corrected estimates of r-square. $\mathrm{P}<0.05$ was considered significant. Software program S-Plus, version 2000, Insightful, Seattle, was used for data analysis.

\section{RESULTS}

The distribution of MRI scores predicting the probability of biochemical recurrence is shown in Table 1. The vast majority of cases, 417 (70\%), were given a score of 2, signifying an MRI report of an organ-confined tumor. Including those patients in whom ECE could not be excluded, 81\% (480) were considered organ-confined with MRI scores from 1 to 3. ECE, both unilateral and bilateral, was predicted in 94 (16\%) cases, while SVI was predicted in 12 (2\%) cases and LNM in $6(1 \%)$ cases.

Comparison of the MRI scores with the pathologic stage showed that 86\% (359/417) of patients with scores of either 1 (no tumor seen) or 2 (tumor seen, no ECE) had no ECE. Of the patients with score 3 (ECE cannot be ruled out), $81 \%$ (50/62) had no ECE. Of the 94 patients predicted to have either unilateral ECE (score 4) or bilateral ECE (score 5), 77 (82\%) had pathological evidence of ECE. Of the 12 patients with score 6 (SVI), 11 (92\%) had SVI, whereas LNM was present in only 3 of 6 predicted cases. Tests for sensitivity and specificity were not calculated, as negative predictions were not included in this model.

Biochemical recurrence occurred in 52 (9\%) patients and the median follow-up for those patients with no evidence of recurrence was 22 months. While only $9 \%$ of the cohort has experienced biochemical recurrence to date, the incidence of recurrence increased as the MRI scores increased: score 1, 0\%; score 2, 5\%; score 3, 10\%; score 4, 15\%; score 5, 22\%; score 6, 58\%; score 7, 67\% (Table 1). 
Table 1. Distribution of cases according to MRI score, with actual recorded events of biochemical recurrence for each score

\begin{tabular}{lccc} 
MRI Score & Description & Distribution & Actual Recurrence \\
\hline 1 & No tumor seen & $1(0.2 \%)$ & 0 \\
\hline 2 & Tumor, no ECE & $417(70 \%)$ & $20(5 \%)$ \\
\hline 3 & Tumor, ECE not excluded & $67(11 \%)$ & $6(10 \%)$ \\
\hline 4 & ECE unilateral & $85(14 \%)$ & $13(15 \%)$ \\
\hline 5 & ECE bilateral & $9(1.5 \%)$ & $2(22 \%)$ \\
7 & SVI & $12(2 \%)$ & $7(58 \%)$ \\
\hline
\end{tabular}

Using the post-operative nomogram results for freedom from recurrence as the endpoint to be predicted preoperatively, we found that prediction was significantly better when the MRI scores were combined with the preoperative nomogram in a single model than when prediction was based on either the MRI scores or the preoperative nomogram alone (Table 2). For the 10-year model to predict freedom from recurrence, the R-square values for the pre-operative nomogram and MRI scoring system were 0.53 and 0.28 , respectively. Combination of these two models resulted in a significant increase in the R-square value to $0.58(p<0.01)$.

We performed the same analysis on those patients who had specific pre-operative variables and were considered to be at higher risk of recurrence. These patients had Gleason score $\geq 7$ on biopsy, clinical stage T2, or PSA $\geq 10$, comprising $66 \%$ of the total study cohort. Similarly to the analysis for the entire cohort, the analysis for this group of high-risk patients showed that the addition of the MRI scores to the pre-operative nomogram resulted in a significant increase in the R-square values for the 10-year prediction model, from 0.58 to 0.61 $(p<0.01)$ (Table 3).

Table 2. R-square values for pre-operative 10-year nomogram, for associated MRI score, and for combinations of both in predicting the biochemical recurrence-free probabilities derived from post-operative nomogram

\begin{tabular}{lc} 
Model & 10-year preoperative nomogram (R-square) \\
\hline Pre-op nomogram & 0.53 \\
MRI-score & 0.28 \\
Combination & $0.58(p<0.01)^{*}$ \\
\end{tabular}

${ }^{*}$ comparison of combination versus pre-op nomogram

Table 3. R-square values for pre-operative 10-year nomogram, for associated MRI score, and for combinations of both in predicting the biochemical recurrence-free probabilities derived from post-operative nomogram in patients at higher risk of recurrence (clinical stage T2, biopsy Gleason score $\geq 7$ or PSA $>10$ )

\begin{tabular}{lc} 
Model & 10-year preoperative nomogram (R-square) \\
\hline Pre-op nomogram & 0.58 \\
MRl-score & 0.25 \\
Combination & $0.61(p<0.01)^{*}$ \\
\end{tabular}

${ }^{*}$ comparison of combination versus pre-op nomogram 


\section{Discussion}

Our study demonstrates that the addition of MRI information to a pre-operative nomogram that includes only clinical stage by digital rectal examination, Gleason grade and PSA significantly improves the accuracy of prediction of freedom from biochemical recurrence. While the improvements in the R square values are modest, it should be noted that the $\mathrm{R}$ square values for the pre-operative nomograms themselves are modest. This is in contrast to the area under the receiver operating characteristic (ROC) curve, 0.75 , that the pre-operative nomogram attains.[13] The limitation of this study is that we used the post-operative nomogram prediction as the endpoint to be predicted preoperatively, rather than trying to predict actual recurrence; this was necessary due to the small size of our dataset and the limited follow-up information available. While high MRI scores were associated with a substantial risk of actual recurrence (Table 1), there were too few recurrence events in our patient cohort to assess whether adding MRI findings to the pre-operative nomogram increased its predictive accuracy based on actual events. Consequently, we used the post-operative nomogram prediction to estimate the probability of recurrence.

A number of clinical and pathological findings have been reported to correlate with risk of recurrence. The degree of differentiation of a tumor, as given by the Gleason score, is a strong predictor of outcome and has been incorporated into nomograms that predict pathologic stage and risk of recurrence. However, there is often a disparity between the biopsy and final pathological Gleason scores. As many as 54\% of cases have a higher grade in the RP specimen than in the biopsy.[14] The design of the MRI scoring system in our study was based on predicted pathologic stage, with a higher score assigned to each increase in stage. It has been shown that higher pathologic stages are associated with higher rates of biochemical recurrence.[15,16] The 10-year biochemical recurrence-free probabilities for patients with organ-confined disease, ECE, SVI and LNM were 92\%,71\%, 37\% and 7\%, respectively.[15] Each of these parameters was a significant predictor of recurrence on multivariate analysis, and the ability of MRI to successfully identify each of these parameters has been reported.[1-3,15]

A meta-analysis by Engelbrecht et al examining the performance of MRI in staging prostate cancer from 1984 to 2000 revealed an accuracy variance from 50\% to 92\%.[8] Some of this variation may be explained by a recent study by Mullerad et al which showed the importance of using dedicated radiologists to analyze prostate images. The study demonstrated that genitourinary MR radiologists were significantly better at predicting ECE on MRI than general body MR specialists.[1]

The ability to diagnose SVI was reported by Bernstein et al, who reviewed 124 clinical T1c patients who were imaged by MRI prior to surgery.[17] While by multivariate analysis MRI did not predict ECE, MRI and pre-operative PSA levels were predictors of SVI. In a recent study comparing pre-operative MRI findings to the pathological specimens from radical prostatectomy for 411 consecutive patients, the positive and negative predictive values of MRI for SVI were reported to be $78 \%$ and $94 \%$, respectively.[2]

The presence of LNM is associated with very low rates of freedom from biochemical recurrence. The conventional imaging criterion for the diagnosis of LNM is a nodal length of $7 \mathrm{~mm}$. Given the shortcomings (demonstrated in our study) of present imaging modalities in predicting LNM, MR lymphography is an exciting prospect. Initial work reported a sensitivity of 100\% and a specificity of 80\%.[18] Harisinghani et al reported on 334 histologically assessed lymph nodes, of which 63 (19\%) nodes from 33 (41\%) patients had metastatic deposits; the sensitivity of MR lymphography was $91 \%$ compared with $35 \%$ for conventional MRI. The main advantage of MR lymphography is its ability to detect metastatic deposits in normal-sized nodes, which is important given that $75 \%$ of all the positive nodes were small enough to not meet the usual MRI radiological criterion.[3]

D'Amico et al investigated the addition of MRI to pre-operative variables to predict recurrence. A single MRI variable (MRI stage T3 versus MRI stage T2) was assessed and was found to be useful in only 19\% of the cohort-i.e., those patients with a PSA between 10 and $20 \mathrm{ng} / \mathrm{mL}$, a biopsy Gleason score $\leq 7$ and a percentage of positive biopsies between $34 \%$ and $50 \%$. In that sub-group, there was a two-fold increase in risk of recurrence from MRI stage T2 to MRI stage T3.[19] The paper demonstrated the benefit, albeit in a minority of patients, of using MRI. In our study, we have extended the MRI predictions from a binary to a 7-point linear scale and included them in a nomogram that does not subdivide patients into risk categories. 
The value of MRI for predicting recurrence has also been studied using artificial neural networks (ANN). Poulakis et al. demonstrated that the combined use of pre-operative variables and pelvic coil MRI was superior to the Kattan nomogram in predicting recurrence.[20] They found that removing clinical stage from the model only marginally reduced the area under the ROC curve (0.897 versus 0.895). However, the use of ANN does not appear to have universal acceptance, and endorectal MRI has been shown to be superior to pelvic MRI and so might increase the accuracy of MRI predictions made with ANN.[21]

In this preliminary study, we have demonstrated that MRI information can be incorporated into a pre-operative nomogram to significantly improve the prediction of biochemical recurrence-free probabilities. Further time is required to allow direct comparison with actual events and to investigate the utility of adding MR spectroscopic imaging findings to the model once sufficient data on recurrence has been accrued. Similarly, the development of a weighted scoring system is desirable, as recurrence rates do not follow a linear pattern when compared to pathologic stage or Gleason score.

\section{REFERENCES}

1. Mullerad M, Hricak H, Wang L, et al: Prostate Cancer: Detection of Extracapsular Extension by Genitourinary and General Body Radiologists at MR Imaging. Radiology 232:140-6, 2004

2. Wang L, Hricak H, Kattan MW, et al: Incremental value of endorectal MRI to the Kattan nomogram in the prediction of seminal vesicle invasion of prostate cancer. Radiology; in press.

3. Harisinghani MG, Barentsz J, Hahn PF, et al: Noninvasive detection of clinically occult lymph-node metastases in prostate cancer. N Engl J Med 348:2491-9, 2003

4. National Comprehensive Cancer Network. Clinical Practice Guidelines in Oncology: Prostate Cancer. Version 2. 2005

5. Hricak H, Wang L, Wei DC, et al: The role of preoperative endorectal magnetic resonance imaging in the decision regarding whether to preserve or resect neurovascular bundles during radical retropubic prostatectomy. Cancer 100:2655-63, 2004

6. Coakley FV, Eberhardt S, Kattan MW, et al: Urinary continence after radical retropubic prostatectomy: relationship with membranous urethral length on preoperative endorectal magnetic resonance imaging. J Urol 168:1032-5, 2002

7. Coakley FV, Eberhardt S, Wei DC, et al: Blood loss during radical retropubic prostatectomy: relationship to morphologic features on preoperative endorectal magnetic resonance imaging. Urology 59:884-8, 2002

8. Engelbrecht MR, Jager GJ, Laheij RJ, et al: Local staging of prostate cancer using magnetic resonance imaging: a meta-analysis. Eur Radiol 12:2294-302, 2002

9. Han M, Partin AW, Pound CR, et al: Long-term biochemical disease-free and cancer-specific survival following anatomic radical retropubic prostatectomy. The 15-year Johns Hopkins experience. Urol Clin North Am 28:555-65, 2001

10. Stephenson AJ, Scardino PT, Eastham JA, et al: Postoperative nomogram predicting the 10-year probability of prostate cancer recurrence after radical prostatectomy. J Clin Oncol 23:7005-12, 2005

11. Aihara M, Wheeler TM, Ohori M, et al: Heterogeneity of prostate cancer in radical prostatectomy specimens. Urology 43:60-6; discussion 66-7, 1994

12. Kattan MW, Eastham JA, Stapleton AM, et al: A preoperative nomogram for disease recurrence following radical prostatectomy for prostate cancer. J Natl Cancer Inst 90:766-71, 1998

13. Graefen M, Karakiewicz PI, Cagiannos I, et al: International validation of a preoperative nomogram for prostate cancer recurrence after radical prostatectomy. J Clin Oncol 20:3206-12, 2002

14. Cookson MS, Fleshner NE, Soloway SM, et al: Correlation between Gleason score of needle biopsy and radical prostatectomy specimen: accuracy and clinical implications. J Urol 157:559-62, 1997

15. Hull GW, Rabbani F, Abbas F, et al: Cancer control with radical prostatectomy alone in 1,000 consecutive patients. J Urol 167:528-34, 2002

16. Han M, Partin AW, Zahurak M, et al: Biochemical (prostate specific antigen) recurrence probability following radical prostatectomy for clinically localized prostate cancer. J Urol 169:517-23, 2003

17. Bernstein MR, Cangiano T, D'Amico A, et al: Endorectal coil magnetic resonance imaging and clinicopathologic findings in T1C adenocarcinoma of the prostate. Urol Oncol 5:104-107, 2000

18. Bellin MF, Roy C, Kinkel K, et al: Lymph node metastases: safety and effectiveness of MR imaging with ultrasmall superparamagnetic iron oxide particles--initial clinical experience. Radiology 207:799-808, 1998

19. D'Amico AV, Whittington R, Malkowicz B, et al: Endorectal magnetic resonance imaging as a predictor of biochemical outcome after radical prostatectomy in men with clinically localized prostate cancer. J Urol 164:759-63, 2000

20. Poulakis $V$, Witzsch $U$, de Vries $R$, et al: Preoperative neural network using combined magnetic resonance imaging variables, prostate specific antigen, and Gleason score to predict prostate cancer recurrence after radical prostatectomy. Eur Urol 46:571-8, 2004

21. Yu KK, Hricak H, Alagappan R, et al: Detection of extracapsular extension of prostate carcinoma with endorectal and phased-array coil MR imaging: multivariate feature analysis. Radiology 202:697-702, 1997 
그를

ssment of Biological

Aggressiveness of Prostate

Cancer: Correlation of MR Signal

Intensity with Gleason Grade on

Whole-Mount Step-Section

Pathologic Analysis after Radical Prostatectomy 


\section{Assessment of Biological Aggressiveness of Prostate Cancer: Correlation of MR Signal Intensity with Gleason Grade on Whole-Mount Step-Section Pathologic Analysis after Radical Prostatectomy}

\section{Supported by National Institutes of Health grant R01 CA76423}

Liang Wang, Yousef Mazaheri, Jingbo Zhang, Nicole M. Ishill, Kentaro Kuroiwa, Hedvig Hricak Departments of Radiology, Medical Physics, Epidemiology and Biostatistics, and Urology Memorial SloanKettering Cancer Center, 1275 York Avenue New York, New York, 10021, USA; Department of Urology, Graduate School of Medical Sciences; Kyushu University, Maidashi, Higashi-ku, Fukuoka 812-8582, Japan

\section{Abstract}

Purpose: To investigate whether the signal intensity (SI) of prostate cancer on T2-weighted MR images correlates with the Gleason grade on whole-mount step-section pathology after radical prostatectomy (RP).

Methods: This IRB-approved, HIPAA-compliant study included 74 patients who underwent endorectal MRI before RP with subsequent whole-mount step-section pathology between January 2001 and July 2004 and had no prior treatment; at least one lesion with bi-dimensional diameter product $\geq 20 \mathrm{~mm}^{2}$ of uniform Gleason grade 3 or 4 or with Gleason grade 5 components; and no high SI indicating postbiopsy changes on T1-weighted images. Sls of prostate tumors, non-tumor prostatic tissue and internal obturator muscles were measured on uncorrected and corrected T2-weighted images. Correlations between Gleason grades and SI ratios (SIRs) were assessed using Kendall's Tau-b. SIRs in peripheral zone (PZ) and transition zone (TZ) lesions of the same Gleason grade were compared using an unpaired t-test.

Results: Seventy-nine Gleason grade 3, 8 Gleason grade 4, and 4 Gleason grade 4/5 lesions identified at pathology were analyzed. Gleason grade correlated significantly $(p<0.05)$ with tumor-to-muscle SIR for PZ and TZ tumors on corrected (Tau- $b=-0.50$ and -0.35 , respectively) and uncorrected (Tau-b $=-0.51$ and -0.33 , respectively) images. Higher Gleason grades were associated with lower tumor-to-muscle SIRs. Non-tumor-to-muscle SIRs did not correlate with patients' Gleason grades. Tumor-to-muscle SIRs were lower in TZ than in PZ tumors $(p<0.001)$.

Conclusion: Higher Gleason grades were associated with lower tumor-to-muscle SIRs on T2-weighted MRI. SI evaluation on T2-weighted MRI may facilitate noninvasive assessment of prostate cancer aggressiveness. 


\section{INTRODUCTION}

rostate cancer is a histologically heterogeneous and frequently multifocal disease $(1,2)$. Its biologic aggres-
siveness varies greatly and is a key predictor of outcome $(2-5)$. The assessment of prostate cancer aggressive-
ness can help stratify patients for appropriate treatment $(1,6)$.

Since its initial description 40 years ago, the Gleason grading system has been the gold standard for measuring prostate cancer biological aggressiveness $(7,8)(9)$. Gleason grades range from 1 to 5 , indicating gradations from well to poorly differentiated prostate cancer. When evaluating tumor aggressiveness, the pathologist assigns both a primary and a secondary Gleason grade. The primary grade is the pattern of cancer that is most prevalent; the secondary grade is the pattern of cancer that is the second most prevalent and that accounts for more than $5 \%$ of the examined specimen (if no pattern of cancer meets the latter criteria, the secondary grade is the same as the primary grade). As the Gleason grade increases, the likelihood of disease recurrence becomes greater $(7,8,10)$.

Endorectal coil magnetic resonance (MR) imaging is gaining acceptance as a tool for the noninvasive detection, localization and staging of prostate cancer $(1,11,12)$. It has been established that prostate cancer typically demonstrates lower signal intensity than healthy prostate tissue on T2-weighted MR imaging (13) (14). In addition, one study found that quantitative measurements of signal intensity on T2-weighted MR imaging, combined with consideration of PSA levels, could improve differentiation between benign peripheral zone tissue and prostate cancer (15). Although metabolic data from MR spectroscopic imaging has been shown to correlate with Gleason grade (16), to the best of our knowledge, no previous studies have examined whether signal intensity on MRI might also correlate with Gleason grade. The purpose of our study was to investigate whether the signal intensity of prostate cancer on T2-weighted MR images correlates with the Gleason grade on whole-mount step-section pathology after radical prostatectomy.

\section{Materials and Methods}

\section{Subjects}

Between January 2001 and July 2004, 74 patients (median age, 57.5 years; age range, 32-72 years, median weight, $86.6 \mathrm{~kg}$; weight range, 59-125 kg) underwent endorectal MRI followed by radical prostatectomy with subsequent whole-mount step-section pathology and met the following criteria for inclusion in our study: no treatment received before radical prostatectomy; at least one tumor of uniform Gleason grade 3 or 4 or with Gleason grade 5 components, with a bi-dimensional diameter product of at least $20 \mathrm{~mm}^{2}$; and no high signal intensity indicative of postbiopsy changes on T1-weighted images. From October 2000 through June 2003, imaging occurred as part of an ongoing National Institutes of Health $(\mathrm{NIH})$ study investigating the use of MR in patients with prostate cancer. All patients gave informed consent before enrollment in the prospective NIH study, which was compliant with the Health Insurance Portability and Accountability Act (HIPAA). From July 2003 to July 2004, prostate MR imaging occurred as part of clinical practice for patient evaluation. For all patients, our institutional review board issued a waiver of authorization for the retrospective review of the MRI exams and clinical data, which was also HIPAA-compliant. The median time from biopsy to MRI was 35 days (range: 32 days before biopsy to 189 days after biopsy) and the median time from MRI to surgery was 29 days (range: 1 day to 164 days). All patients had a tissue diagnosis of prostate cancer on biopsy specimens. Age, weight, serum PSA level, and clinical stage were recorded from the patients' medical records (--,--).

\section{MR imaging technique}

Endorectal MRI was performed on a GE 1.5-Tesla whole-body magnetic resonance (MR) scanner equipped with both a pelvic phased-array coil (GE Medical systems, Milwaukee, WI) and a commercially available balloon-covered expandable endorectal coil (Medrad, Pittsburgh, PA) for signal reception. Patients were examined in the supine position. T1-weighted axial and spin-echo images were obtained from the aortic bifurcation to the symphysis pubis using the following parameters: $\mathrm{TR}=400-700 \mathrm{msec}, \mathrm{TE}=10-14 \mathrm{msec}$, slice thickness $=5 \mathrm{~mm}$, inter-slice gap $=1 \mathrm{~mm}$, field of view $(F O V)=24-26 \mathrm{~cm}$, matrix $256 \times 192$, and 1 excitation. Thin-section, high spatial resolution axial, coronal, and sagittal T2-weighted fast spin-echo images of the prostate and seminal vesicles were obtained using the following parameters: TR = 3650-6917 msec, effective TE = 78-135 msec, echo train length $=8$-32, slice thickness = 3-5 mm, inter-slice gap $=0 \mathrm{~mm}$, and FOV $=14-20 \mathrm{~cm}$, with a matrix of 256-320 $\times 192$. The total time for setup and acquisition was approximately 30 minutes. 
Endorectal coil profiles were obtained from a phantom study performed by a medical physicist (--, 6 years of experience in MR research) and a radiologist (--6 years of research experience in prostate MR imaging) to identify signal intensity iso-surface on T2-weighted images. A spherical phantom consisting of saline doped with T1-shortening contrast agent to yield $\mathrm{T} 1$ values $\sim 1 \mathrm{sec}$ was surrounded with saline bags to mimic the typical loading of a patient and was imaged with the endorectal coil setup. Corrected and uncorrected axial T2-weighted images were generated similar to those obtained from a prostate exam. Images were exported to Matlab (MathWorks, Natick, MA) and signal intensity iso-surface profiles were generated both in the z-direction and as a function of distance to the endorectal coil in the in-plane direction on T2-weighted images.

Image post-processing was performed with prostatic analytical coil correction (PACC) software (GE Medical Systems, Milwaukee, WI) on the axial T1- and T2-weighted MR images to correct for the reception profile of the endorectal and external pelvic phased-array coils. All MRI data were transferred to the picture archiving and communication system (PACS) (GE Medical Systems, Milwaukee, WI) of our radiology department.

\section{Pathologic Evaluation}

Following prostate resection the specimen was step-sectioned as previously described (17) and reviewed by a pathologist (--, 8 years experience). Cancer foci were outlined in ink on whole-mount, apical, and seminal vesicle slices, so as to be grossly visible, and were then photographed to provide tumor maps. The greatest diameter and the greatest perpendicular diameter for each lesion on the whole-mount step-section maps were measured, and bi-dimensional products were calculated. The locations of tumors were recorded (peripheral zone [PZ] or transition zone [TZ]; base, mid-gland or apex). The pathologic stage and the presence of extraprostatic extension, seminal vesicle invasion, lymph node metastasis, and positive margins were also recorded.

\section{MR image analysis}

MR images and whole-mount pathologic step-section slices were analyzed by a radiologist (--,6 years of research experience in MR imaging) and a pathologist (--, 8 years experience). The whole-mount pathologic step-section slices were matched with the most closely corresponding T2-weighted MR images.

Tumors that had a bi-dimensional diameter product of at least $20 \mathrm{~mm}^{2}$ on whole-mount pathology maps and that were of uniform Gleason grade 3 or 4 or contained Gleason grade 5 components were analyzed; if a patient had more than one such tumor, the two largest were analyzed. On both corrected and uncorrected axial T2-weighted images, a round or elliptical region of interest (ROI) (area: 4-10 $\mathrm{mm}^{2}$ ) was placed on the center of the tumor using cross-referencing on PACS with reference to the pathological maps. When a tumor extended through both the PZ and the TZ, two separate ROIs were placed, one in the PZ and one in the TZ portion of the tumor. The ROls were placed so as not to include the prostate capsule or urethra. Mean signal intensities and standard deviations in the $\mathrm{ROI}$ were automatically calculated by PACS. For normalization, ROI measurements in non-tumor prostatic tissue and the internal obturator muscle were performed on both corrected and uncorrected images. To correct for signal inhomogeneity from the endorectal coil, two approaches were used for evaluating signal intensities on corrected and uncorrected images. On uncorrected T2-weighted axial images, the mapped iso-surface profile obtained from the phantom study was used to guide ROI selection. Round or elliptical ROIs (area: 4-10 mm²) were placed on nontumor prostatic tissue and internal obturator muscle along the signal intensity iso-surface of the tumor ROI. On T2-weighted axial images corrected with PACC software, ROls were placed on contralateral non-tumor prostatic tissue symmetrically to the tumor $\mathrm{ROI}$ and on internal obturator muscle as close to the tumor as possible (distance range: 6 - $28 \mathrm{~mm}$ ). The mean signal intensities measured by the ROls were used to calculate the tumor-to-muscle and non-tumor-to-muscle signal intensity ratios (SIRs).

\section{Statistical analysis}

On both the uncorrected and corrected images, Kendall's Tau-b was used to examine correlations between Gleason grades and tumor-to-muscle and non-tumor-to-muscle SIRs. An unpaired t-test was used to test for differences in tumor-to-muscle and non-tumor-to-muscle SIRs between PZ and TZ lesions of the same Gleason grade. In all statistical methods, a P value of less than .05 was considered to indicate significant difference. Analyses were performed with Intercooled Stata 8.0 for Windows (Stata Corporation, College Station, TX, 2003) and SAS for Windows 9.0 (SAS Institute, Cary, NC 2002). 


\section{RESULTS}

\section{Patient and Lesion Characteristics}

Patient characteristics are summarized in Table 1. Seventy-four patients with a total of 91 lesions were included in the study. Seventeen patients each had two lesions analyzed. Fifty-nine (65\%) of the 91 lesions were in the PZ and 32 (35\%) were in the TZ. Seventy-nine of the lesions (87\%) were Gleason grade 3, 8 (9\%) were Gleason grade 4, and 4 (4\%) were mixed Gleason grade 4/5. At whole-mount step-section pathology, the median lesion diameter was 16 $\mathrm{mm}$ (range 5-48 mm), the median greatest perpendicular diameter was $8 \mathrm{~mm}$ (range 4-38 mm), and the median bi-dimensional product was $144 \mathrm{~mm}$ (20-1824 mm). Figure 1 shows the endorectal profile generated from the phantom study, and Figure 2 shows how ROI measurements were performed in a typical case. Table 2 lists the median values and ranges for the SIRs measured on the uncorrected and corrected images.

\section{Correlation of Signal intensity and Gleason score}

\section{A. Uncorrected Images}

Gleason grade correlated significantly with tumor-to-muscle SIR for both PZ and TZ tumors (Tau-b $=-0.51, p<0.001$ and Tau- $b=-0.33, p=0.03$ respectively), with higher Gleason grade tumors demonstrating lower SIRs (Table 3; Figs. 2,3, and 4). The non-tumor-to-muscle SIRs in the PZ did not differ significantly across patients with different Gleason grades (Tau-b $=-0.06, p=0.57$ ), indicating that no significant bias existed in our measurements (Table 3 ).

\section{Table 1. Patient Characteristics}

\begin{tabular}{|ccc|} 
& Median & Range \\
\hline Age & 57.5 & $37-72$ \\
\hline Weight (kg) & 86.6 & $59-125$ \\
\hline Pre RP PSA & 5.05 & $0.8-76.8$ \\
\hline & N & $\%$ \\
\hline Clinical Stage & & \\
\hline T1 & 47 & 64 \\
\hline T2 & 25 & 34 \\
\hline T3 & 2 & 3 \\
\hline Path Stage & & 78 \\
\hline T2 & 58 & 20 \\
\hline T3 & 15 & 1 \\
\hline T4 & 1 & 22 \\
\hline ECE & 16 & 4 \\
\hline SVI & 3 & 5 \\
\hline Positive Margins & 4 & \\
\hline & 12 & \\
\hline
\end{tabular}

Note: PSA = serum prostate-specific antigen; PNI = perineural invasion on biopsy; $\mathrm{ECE}=$ Extraprostatic extension; $\mathrm{SVI}=$ seminal vesicle invasion; $\mathrm{LNM}=$ lymph node metastasis; $\mathrm{RP}=$ radical prostatectomy 

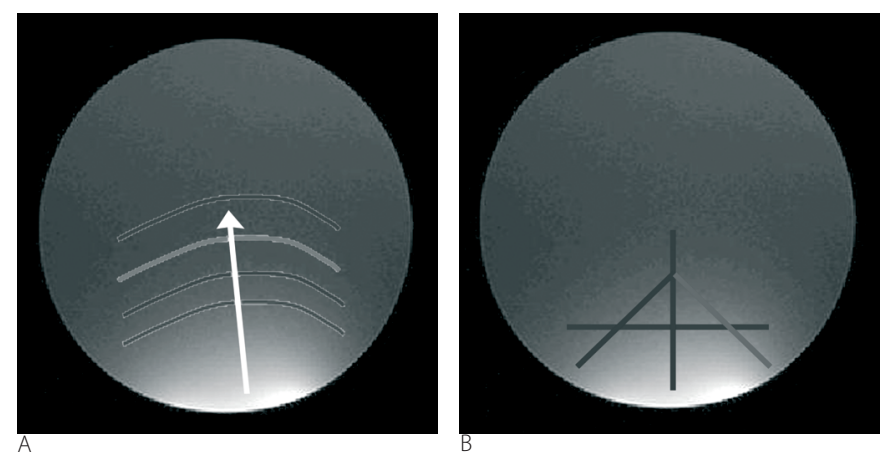

Figure 1: Generating iso-surface image profiles from the phantom study. (a) and (b) show the iso-surface and projections on a phantom, respectively; (c) and (d) show the corresponding signal intensity profiles for the configurations in (a) and (b). Signal intensity from the mid $1 / 3$ of the balloon-covered expandable endorectal coil remained homogeneous in the $z$ dimension (e).

(Color version available in chapter 18).
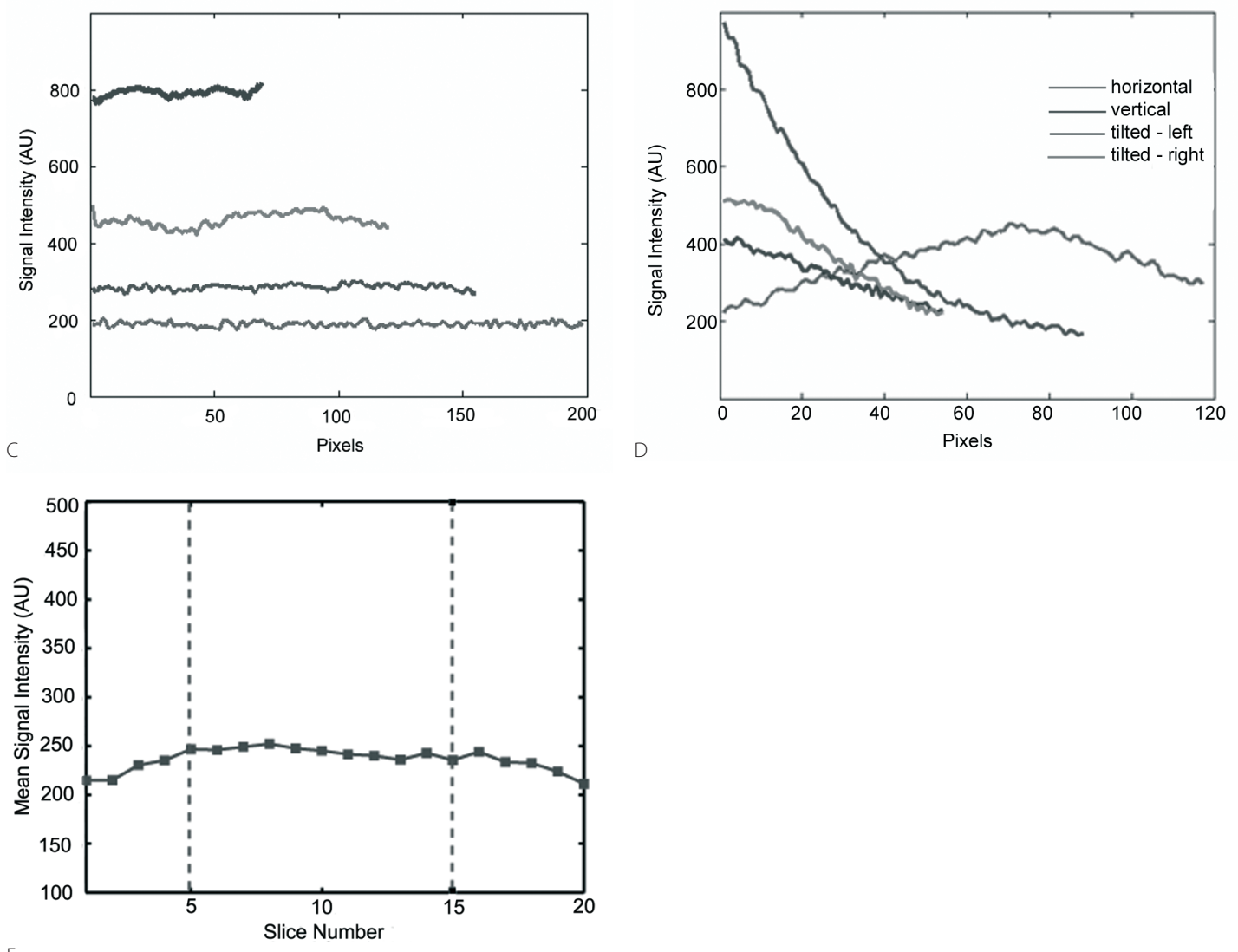

$E$

\section{B. Corrected Images}

Gleason grade correlated significantly with tumor-to-muscle SIR for both PZ and TZ tumors (Tau-b $=-0.50, p<0.001$ and Tau-b $=-0.35, p=0.02$ respectively) (Table 3; Fig. 4), with higher Gleason grade tumors demonstrating lower SIRs. The non-tumor-to-muscle SIRs demonstrated no significant difference across patients with different Gleason grades in the PZ (Tau- $b=-0.02, p=0.83$ ) or in the TZ (Tau-b $=-0.26, p=0.11$ ), indicating that no significant bias existed in our measurements (Table 3).

\section{TZ tumor vs. PZ tumor}

\section{A. Uncorrected Images}

Gleason grade 3 lesions in the TZ had a significantly lower $(p<0.001)$ mean tumor-to-muscle SIR than Gleason grade 3 lesions in the PZ (Table 3, Figs. 3 and 4). The numbers of Gleason grade 4 and 4/5 lesions in the TZ were too small for formal analysis. 


\section{B. Corrected Images}

Gleason grade 3 lesions in the TZ had a significantly lower ( $p<0.001$ ) mean tumor-to-muscle SIR than Gleason grade 3 lesions in the PZ (Table 3, Figs. 3 and 4).

Table 2. Median and range of signal intensity ratios on uncorrected images and corrected images

\begin{tabular}{lcc} 
Uncorrected images & Median & Range \\
\hline Tumor-to-muscle SIR & 3.57 & $1.22-9.66$ \\
\hline Tumor-to-non-tumor SIR & 0.67 & $0.22-3.91$ \\
\hline Non-tumor-to-muscle SIR & 5.93 & $1.0-17.6$ \\
\hline Corrected images & & \\
\hline Tumor-to-muscle SIR & 2.42 & 0.75 \\
\hline Tumor-to-non-tumor SIR & 0.69 & $0.22-1.01$ \\
\hline Non-tumor-to-muscle SIR & 3.82 & $1.79-12.07$ \\
\hline
\end{tabular}

Notes: SIR = signal intensity ratio

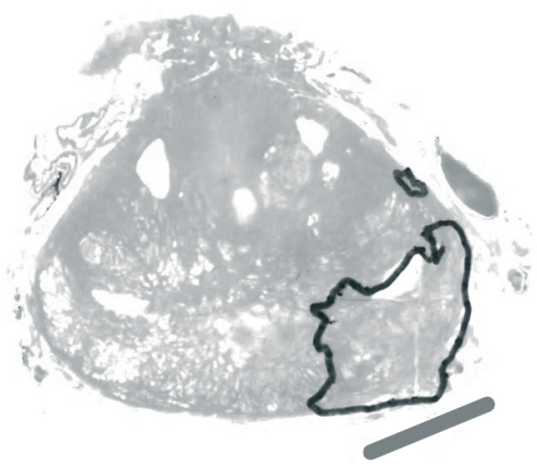

A

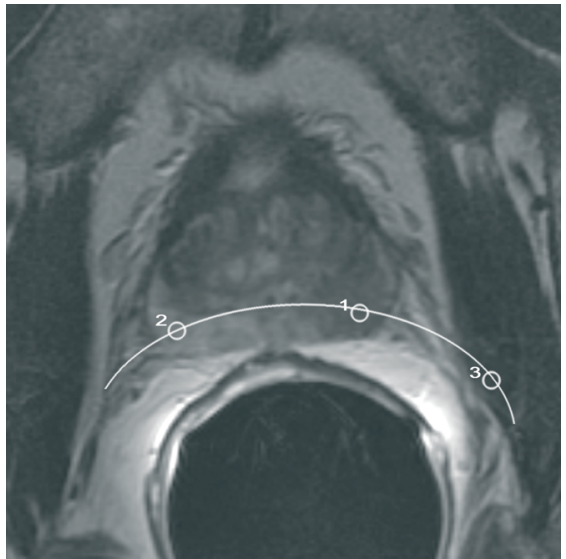

B
Figure 2: 54-year-old male with Gleason grade 4 prostate cancer in the peripheral zone (PZ) (weight $100.2 \mathrm{~kg}$, PSA $4.4 \mathrm{ng} / \mathrm{ml}$, clinical stage T2b, pathologic stage T3b). (a) Whole-mount step-section pathologic tumor map shows an index lesion of Gleason grade 4 in left PZ. The black circle represents the area of Gleason grade 4. (b) On the corresponding transverse uncorrected T2-weighted MR image (repetition time msec/echo time msec, 4916.7/102.48; echo train length, 12; field of view, $14 \mathrm{~cm}$; section thickness, $3 \mathrm{~mm}$; no section gap) ROls were placed on the center of the tumor (ROI-1) on non-tumor prostatic tissue (ROI-2) and on internal obturator muscle (ROI-3). The PZ tumor-to-muscle SIR was 2.27. The PZ non-tumor prostatic tissue-tomuscle SIR was 4.86. (c) On the corresponding transverse T2-weighted MR image corrected with prostatic analytical coil correction (PACC) software, ROls were placed the center of the tumor using (ROI-4) on contralateral non-tumor prostatic tissue (ROI-5) and on internal obturator muscle (ROI-6). The PZ tumor-to-muscle SIR was 1.82. The PZ non-tumor prostatic tissue-to-muscle SIR was 3.44 .

(Color version available in chapter 18).

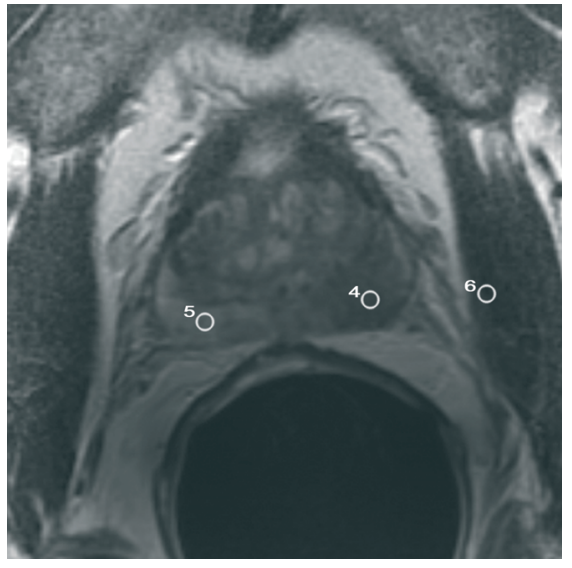

C 
Table 3. Mean tumor-to-muscle signal intensity ratios for peripheral zone and transition zone tumors by Gleason grade for (a) the uncorrected images and $(b)$ the corrected images.

\begin{tabular}{llcccc} 
A Uncorrected images & N & Mean T/M SIR (SD) & Tau-b & P value* $^{*}$ \\
\hline PZ Gleason grade 3 & 49 & $5.3(1.65)$ & \\
\hline PZ Gleason grade 4 & 7 & $2.9(0.60)$ & -0.51 & $<0.001$ \\
\hline PZ Gleason grade 5 & 3 & $2.05(0.56)$ & & \\
\hline TZ Gleason grade 3 & 30 & $2.7(0.65)$ & -0.33 & 0.03 \\
\hline TZ Gleason grade 4 & 1 & 1.66 & &
\end{tabular}

\begin{tabular}{llcccc} 
Corrected images & N & Mean T/M SIR (SD) & Tau-b & P value* $^{*}$ \\
\hline PZ Gleason grade 3 & 49 & $3.4(1.2)$ & \\
\hline PZ Gleason grade 4 & 7 & $1.9(0.40)$ & -0.50 & $<0.001$ \\
PZ Gleason grade 5 & 3 & $1.2((0.41)$ & & \\
\hline TZ Gleason grade 3 & 30 & $2.1(0.50)$ & -0.35 & 0.02 \\
TZ Gleason grade 4 & 1 & 1.07 & &
\end{tabular}

Notes: $\mathrm{PZ}=$ peripheral zone; $\mathrm{TZ}=$ transition zone; $\mathrm{SD}=$ standard deviation $\mathrm{T} / \mathrm{M} \mathrm{SIR}=$ tumor-to-muscle signal intensity ratio
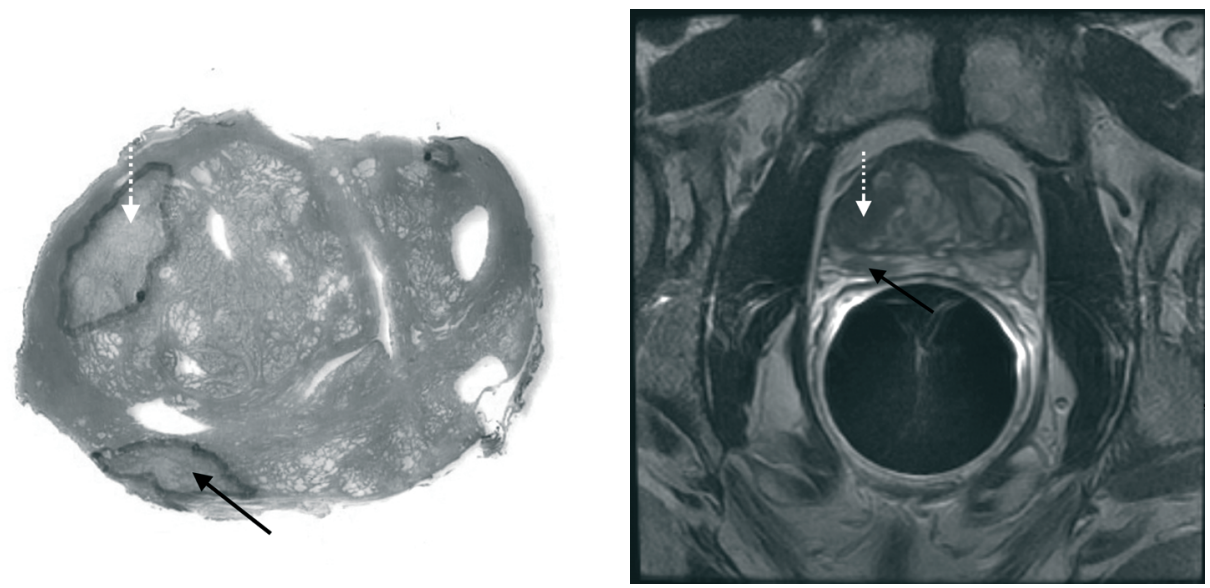

A

Figure 3: 60-year-old male with Gleason grade 3 prostate cancers in the PZ and TZ (weight $80.7 \mathrm{~kg}$, PSA $5.1 \mathrm{ng} / \mathrm{ml}$, clinical stage T2b, pathologic stage T2c). (a) Whole-mount step-section pathologic tumor map shows an index lesion of Gleason grade 3 in the right TZ (dashed white arrow), and a second dominant lesion of Gleason grade 3 (black arrow) in the right posterior PZ. The outlined areas represent the two tumors. (b) Corresponding transverse T2-weighted MR image (TR/effective TE = 4000/103; echo-train length, 16; field of view, $14 \mathrm{~cm}$; acquisition matrix, $256 \mathrm{x}$ 192; section thickness, $3 \mathrm{~mm}$; no section gap) corrected with PACC software shows the index Gleason grade3 lesion (dashed white arrow) in the $T Z$, and the second dominant Gleason grade 3 lesion (black arrow) in the PZ. The tumor-to-muscle SIR (1.77) in the TZ is lower than that in the PZ (3.99). Also note that the PZ tumor-to-muscle SIR in this Gleason grade 3 lesion (3.99) is higher than that of the Gleason grade 4 lesion (1.82) in a different patient shown in Figure 2 .

(Color version available in chapter 18). 


\section{Base tumor vs. mid-gland tumor vs. apical tumor}

\section{A. Uncorrected Images}

The majority of Gleason grade 3 tumors were in the mid-gland and apex. The mean tumor-to-muscle SIRs for Gleason grade 3 lesions did not differ significantly between these two regions (Table 4). The numbers of Gleason grade 4 and $4 / 5$ tumors were too small for formal analysis.

\section{B. Corrected Images}

The mean tumor-to-muscle SIRs for Gleason grade 3 lesions did not differ significantly in the mid-gland and the apex of the prostate (Table 4).

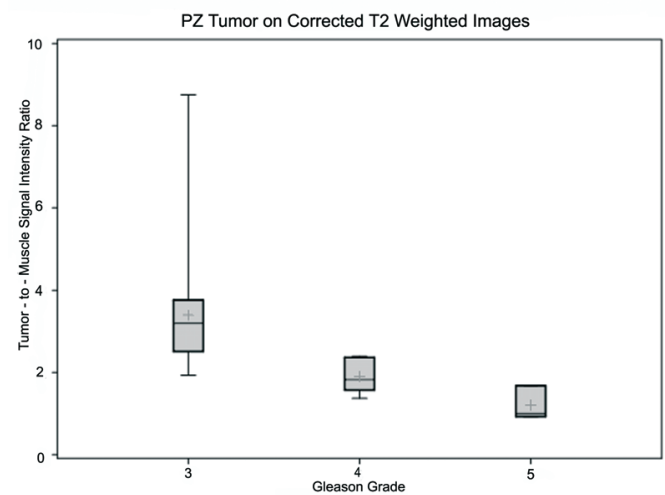

A

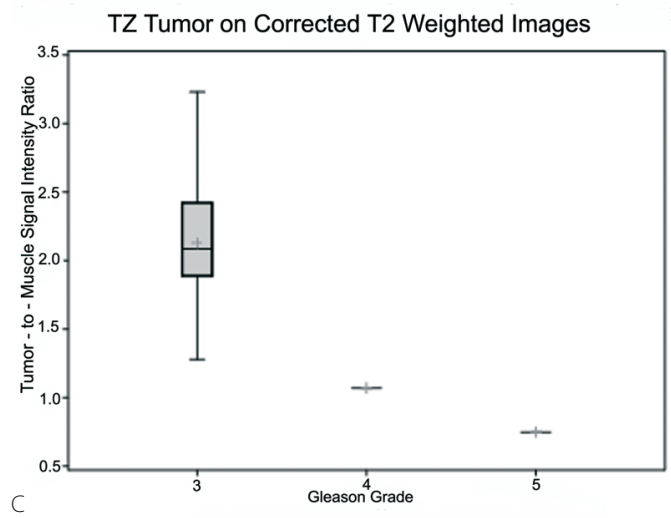

PZ Tumor on Uncorrected T2 Weighted Images

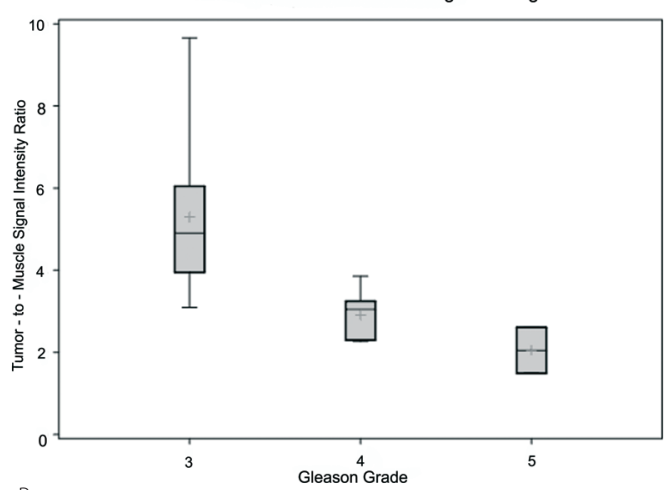

B

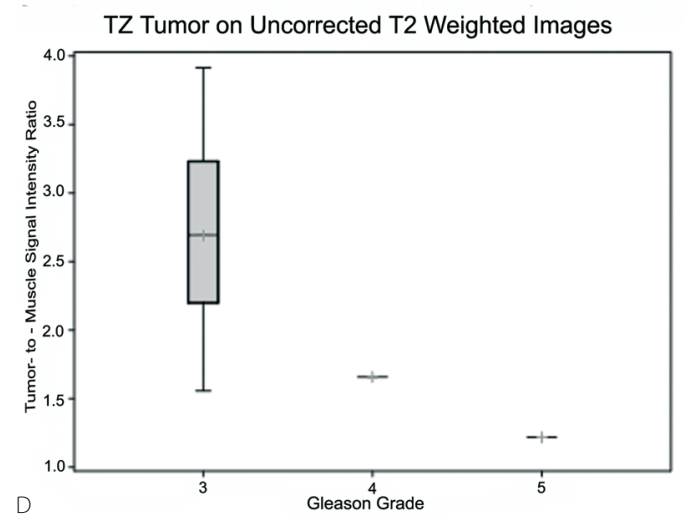

Figure 4: Box plots of tumor-to-muscle signal intensity ratios by Gleason grade in PZ and TZ. (a) PZ tumor on corrected T2 weighted images; (b) PZ tumor on uncorrected T2 weighted images. (c) TZ tumor on corrected T2 weighted images; (d) TZ tumor on uncorrected T2 weighted images. $\mathrm{PZ}=$ peripheral zone; $\mathrm{TZ}=$ transition zone; crosses = mean for that group; box = middle $50 \%$ of values; black line in box = the group median; lines at ends of error bars $=$ minimum $/$ maximum values.

\section{Discussion}

Endorectal MR imaging, with and without MR spectroscopic imaging, has the capacity to aid in the detection, localization, and staging of prostate cancer (18-23). Furthermore, MR spectroscopic imaging may have a role in the evaluation of tumor aggressiveness, since metabolic data from MRSI correlate with prostate cancer Gleason grade (16). However, to the best of our knowledge, the capacity of MRI alone to provide information about tumor aggressiveness has not been explored. Engelhard et al used the SIR on T2-weighted MR imaging and serum PSA level to differentiate benign disease from prostate cancer in the peripheral zone, using needle biopsy histopathological findings as the standard of reference (15). Although their study did not address tumor aggressiveness specifically, it did suggest that quantitative evaluation of SIRs on MRI could provide more information than visual evaluation alone 
Table 4. Mean tumor-to-muscle SIRs by Gleason grade and location (base, mid-gland, and apex of the peripheral zone or transition zone) of tumor within the prostate for (a) the uncorrected images and (b) the corrected images.

\begin{tabular}{|c|c|c|c|c|c|c|c|}
\hline \multirow[t]{9}{*}{ A } & \multirow{2}{*}{$\begin{array}{l}\text { Uncorrected images } \\
\text { Location in PZ }\end{array}$} & \multicolumn{2}{|r|}{ Gleason 3} & \multicolumn{2}{|c|}{ Gleason 4} & \multicolumn{2}{|c|}{ Gleason 5} \\
\hline & & $\mathrm{N}$ & Mean T/M SIR (SD) & $\mathrm{N}$ & Mean (SD) & $\mathrm{N}$ & Mean (SD) \\
\hline & Apex & 21 & $5.6(1.5)$ & 4 & $2.7(0.46)$ & 0 & - \\
\hline & Mid-gland & 25 & $5.3(1.8)$ & 1 & 3.9 & 1 & 2.6 \\
\hline & Base & 3 & $3.8(0.75)$ & 2 & $2.8(0.96)$ & 2 & $1.8(0.39)$ \\
\hline & Location in TZ & $\mathrm{N}$ & Mean T/M SIR (SD) & $\mathrm{N}$ & Mean (SD & $\mathbf{N}$ & Mean (SD) \\
\hline & Apex & 5 & $2.75(0.76)$ & 1 & 1.66 & 0 & - \\
\hline & Mid-gland & 24 & $2.7(0.66)$ & 0 & - & 0 & - \\
\hline & Base & 1 & 2.5 & 0 & - & 1 & 1.22 \\
\hline \multirow[t]{9}{*}{ B } & Corrected images & & Gleason 3 & & Gleason 4 & & leason 5 \\
\hline & Location in PZ & $\mathrm{N}$ & Mean T/M SIR (SD) & $\mathrm{N}$ & Mean (SD) & $\mathrm{N}$ & Mean (SD) \\
\hline & Apex & 21 & $3.4(0.96)$ & 4 & $1.8(0.45)$ & 0 & - \\
\hline & Mid-gland & 25 & $3.5(1.5)$ & 1 & 24 & 1 & 1.7 \\
\hline & Base & 3 & $2.6(0.18)$ & 2 & $2.0(0.22)$ & 2 & $0.96(0.05)$ \\
\hline & Location in TZ & $\mathrm{N}$ & Mean T/M SIR (SD) & $\mathrm{N}$ & Mean (SD & $\mathrm{N}$ & Mean (SD) \\
\hline & Apex & 5 & $2.5(0.74)$ & 1 & 1.07 & 0 & - \\
\hline & Mid-gland & 24 & $2.0(0.42)$ & 0 & - & 0 & - \\
\hline & Base & 1 & 2.1 & 0 & - & 1 & 0.75 \\
\hline
\end{tabular}

Notes: $\mathrm{PZ}=$ peripheral zone; $\mathrm{TZ}=$ transition zone; $\mathrm{SD}=$ standard deviation; $\mathrm{T} / \mathrm{M} \mathrm{SIR}=$ tumor-to-muscle signal intensity ratio

regarding the nature of the tissue in a lesion. We therefore decided to investigate whether a correlation existed between SIRs on T2-weighted MR images and tumor Gleason grade on whole-mount step-section pathology after radical prostatectomy. We found that lower tumor-to-muscle SIRs were associated with higher Gleason grades, although there was some overlap between the SIRs for tumors of different grades, perhaps due to tissue heterogeneity. We also found that for all Gleason grade 3 lesions, mean tumor-to-muscle SIRs were significantly lower in the TZ than in the PZ.

The use of an endorectal coil with a phased-array coil significantly increases the SNR in MR imaging of the prostate and thereby improves tumor detection, localization and staging (15) (24, 25). However, it also creates obstacles to the comparison of signal intensities across subjects, since the signal intensity is substantially higher in regions close to the endorectal coil and drops off rapidly with increasing distance from it (Fig. 1). This signal intensity profile may vary from patient to patient depending on the orientation of the endorectal coil at insertion. Furthermore, there may be signal variations along the $z$ axis of the endorectal coil, and the anterior phased-array coil also contributes some signal. For these reasons, signal intensity cannot be compared directly across MR prostate imaging subjects (26). Two approaches may be used to solve this problem:1) image post-processing to correct for the reception profiles of endorectal and phased array coils (26), which is feasible with commercial software; and 2) acquisition of an endorectal coil profile from a phantom to guide placement of ROls along a uniform signal profile, referred to as an iso-surface. The latter approach does not require image intensity correction. Both approaches were used in our study and showed consistent results, indicating that our findings are unlikely to have been influenced by bias. Even though the phantom study showed that signal intensity from the mid 1/3 of the endorectal coil remained homoge- 
neous in the z dimension (Fig. 1), we performed all ROI measurements for the tumor, non-tumor prostate tissue and internal obturator muscle on the same axial MR image to minimize potential bias.

Another potential source of error in quantifying prostate cancer signal intensity is the partial volume effect, which reduces the accuracy of measurements of small cancers as well as the outer edges of large cancers (27). To avoid the partial volume effect from adjacent non-tumor prostatic tissue, only the two largest lesions in the prostate that met the specified size criteria were analyzed. Furthermore, using cross referencing on PACS, ROls were placed on the centers of tumors to avoid tumor edge effects.

Our analysis was based on Gleason grades assigned at the time of map-making, the dates of which preceded the new consensus on Gleason grading. The new consensus document, published in 2005, was largely based on empiric observation rather than validated data, and therefore we did not feel a strong need to re-evaluate the pathology samples for the present study (9).

One limitation of this study was the exclusion of a number of patients because of technical and clinical issues. Although the time interval between biopsy and MR imaging was required to be at least 4 weeks, some patients still demonstrated substantial biopsy changes on T1-weighted images and corresponding signal loss at T2-weighted imaging, which made their data sets unusable. Other limitations were the relatively small numbers of tumors with uniform Gleason grade 4 or with Gleason grade 5 components in the study population, which prevented us from comparing the SIRs for such tumors in different regions of the prostate.

Our findings suggest that in addition to providing anatomic information for tumor detection, localization and staging, T2-weighted endorectal MRI has the potential to allow noninvasive assessment of prostate cancer biological aggressiveness, which could help stratify patients for appropriate management. Future studies with larger numbers of patients and prospective quantitative T2 measurement may be needed to confirm our findings.

\section{RefERENCES}

1. Hricak H. MR imaging and MR spectroscopic imaging in the pre-treatment evaluation of prostate cancer. Br J Radiol 2005 ; 78 Spec No 2: S103-111.

2. Ohori M, Kattan M, Scardino PT, Wheeler TM. Radical prostatectomy for carcinoma of the prostate. Mod Pathol 2004; 17:349-359.

3. Kattan MW, Wheeler TM, Scardino PT. Postoperative nomogram for disease recurrence after radical prostatectomy for prostate cancer. J Clin Oncol 1999; 17:1499-1507.

4. Epstein Jl, Partin AW, Sauvageot J, Walsh PC. Prediction of progression following radical prostatectomy. A multivariate analysis of 721 men with long-term follow-up. Am J Surg Pathol 1996; 20:286-292.

5. Fryback DG, Albertsen PC, Storer BE. Prostatectomy and survival among men with clinically localized prostate cancer. Jama 1996; 276:1723-1724; author reply 1724.

6. Scardino PT. Continuing refinements in radical prostatectomy: more evidence that technique matters. J Urol 2005; 173:338-339.

7. Gleason DF. Classification of prostatic carcinomas. Cancer Chemother Rep 1966; 50:125-128.

8. Gleason DF, Mellinger GT. Prediction of prognosis for prostatic adenocarcinoma by combined histological grading and clinical staging. J Urol 1974; 111:58-64.

9. Epstein Jl, Allsbrook WC, Jr., Amin MB, Egevad LL. The 2005 International Society of Urological Pathology (ISUP) Consensus Conference on Gleason Grading of Prostatic Carcinoma. Am J Surg Pathol 2005; 29:1228-1242.

10. Epstein J. What's new in prostate cancer disease assessment in 2006? Curr Opin Urol 2006; 16:146-151.

11. Wang L, Mullerad M, Chen HN, et al. Prostate cancer: incremental value of endorectal MR imaging findings for prediction of extracapsular extension. Radiology 2004; 232:133-139.

12. Engelbrecht MR, Jager GJ, Laheij RJ, Verbeek AL, van Lier HJ, Barentsz JO. Local staging of prostate cancer using magnetic resonance imaging: a meta-analysis. Eur Radiol 2002; 12:2294-2302.

13. Hricak $H$, Wang $L$, Wei DC, et al. The role of preoperative endorectal magnetic resonance imaging in the decision regarding whether to preserve or resect neurovascular bundles during radical retropubic prostatectomy. Cancer 2004; 100:2655-2663.

14. Akin O, Hricak H. Imaging of Prostate Cancer. Radiol Clin North Am 2007; 45:207-222.

15. Engelhard K, Hollenbach HP, Deimling M, Kreckel M, Riedl C. Combination of signal intensity measurements of lesions in the peripheral zone of prostate with MRI and serum PSA level for differentiating benign disease from prostate cancer. Eur Radiol 2000; 10:1947-1953.

16. Zakian KL, Sircar K, Hricak H, et al. Correlation of proton MR spectroscopic imaging with gleason score based on step-section pathologic analysis after radical prostatectomy. Radiology 2005; 234:804-814.

17. Yossepowitch $\mathrm{O}$, Sircar K, Scardino PT, et al. Bladder neck involvement in pathological stage pT4 radical prostatectomy specimens is not an independent prognostic factor. J Urol 2002; 168:2011-2015.

18. Huzjan R, Sala E, Hricak H. Magnetic resonance imaging and magnetic resonance spectroscopic imaging of prostate cancer. Nat Clin Pract Urol 2005; 2:434-442.

19. Wang L, Hricak H, Kattan MW, Chen HN, Scardino PT, Kuroiwa K. Prediction of organ-confined prostate cancer: incremental value of MR imaging and MR spectroscopic imaging to staging nomograms. Radiology 2006; 238:597-603. 
20. Futterer JJ, Engelbrecht MR, Jager GJ, et al. Prostate cancer: comparison of local staging accuracy of pelvic phased-array coil alone versus integrated endorectal-pelvic phased-array coils : Local staging accuracy of prostate cancer using endorectal coil MR imaging. Eur Radiol 2006.

21. Wang L, Hricak H, Kattan MW, et al. Prediction of Seminal Vesicle Invasion in Prostate Cancer: Incremental Value of Adding Endorectal MR Imaging to the Kattan Nomogram. Radiology 2007; 242:182-188.

22. Zakian KL, Eberhardt S, Hricak H, et al. Transition zone prostate cancer: metabolic characteristics at $1 \mathrm{H}$ MR spectroscopic imaging--initial results. Radiology 2003; 229:241-247.

23. Shukla-Dave A, Hricak H, Eberhardt SC, et al. Chronic prostatitis: MR imaging and $1 \mathrm{H}$ MR spectroscopic imaging findings--initial observations. Radiology 2004; 231:717-724.

24. Hricak H, White S, Vigneron D, et al. Carcinoma of the prostate gland: MR imaging with pelvic phased array coil versus integrated endorectal-pelvic phased-array coils. . Radiology 1994; 193:703-709.

25. Huch Boni RA, Boner JA, Lutolf UM, Trinkler F, Pestalozzi DM, Krestin GP. Contrast-enhanced endorectal coil MRI in local staging of prostate carcinoma. J Comput Assist Tomogr 1995; 19:232-237.

26. Moyher SE, Vigneron DB, Nelson SJ. Surface coil MR imaging of the human brain with an analytic reception profile correction. J Magn Reson Imaging 1995; 5:139-144.

27. Gonzalez Ballester MA, Zisserman AP, Brady M. Estimation of the partial volume effect in MRI. Med Image Anal 2002; 6:389-405. 
Post Processing of Dynamic Gadolinium-Enhanced MRI Exams

of the Liver: Explanation and Potential Clinical Applications for Color-Coded Qualitative and Quantitative Analysis 


\section{Post Processing of Dynamic Gadolinium-Enhanced MRI Exams of the Liver: Explanation and Potential Clinical Applications for Color-Coded Qualitative and Quantitative Analysis accepted for publication in Acta Radiologica}

Liang Wang, Indra C van den Bos, Shahid M Hussain, Bert van Heerebeek, Peter MT Pattynama, Mika W Vogel, Gabriel P Krestin

Department of Radiology, Erasmus MC, University Medical Center Rotterdam, The Netherlands; and Department of Radiology, University of Nebraska, Medical Center, Omaha, NE, USA, Present address: Department of Radiology, Memorial Sloan-Kettering, Cancer Center, 1275 York Avenue, New York, NY, 10021, USA.

\section{Abstract}

The purpose of this article is to explain and illustrate the current status and potential applications of automated and color-coded post processing techniques for analysis of dynamic multiphasic gadolinium-enhanced MR imaging of the liver. Post processing of these images on dedicated workstations allows generation of time-intensity curves (TIC) as well as color-coded images, which provides useful information on (neo)-angiogenesis within a liver lesion, if necessary combined with information on enhancement patterns of surrounding liver parenchyma. Analysis of TIC and color-coded images provides an easy to interpret schematic presentation of tumor behavior, providing additional characteristics for adequate differential diagnosis. Inclusion of TIC and color-coded images as part of the routine abdominal MR imaging work-up protocol may help to further improve the specificity of MR imaging findings and may facilitate the diagnostic work-up of disease for detection, staging, and monitoring of anti-tumor therapy. 


\section{INTRODUCTION}

$n$ the analysis of liver diseases, dynamic multiphasic gadolinium-enhanced magnetic resonance (MR) imaging is routinely used as part of the imaging protocol, since it provides information on a multitude of parameters,

including information on tumor angiogenesis related to tumor growth, tumor grade, metastatic potential and anti-tumor therapy response (1, 2). Since hepatic perfusion regarding blood flow per tissue unit is essential for analysis and differential diagnosis of either focal or diffuse liver abnormalities, quantification of these parameters may facilitate improved evaluation of hepatic diseases(3).

Analysis of hepatic enhancement is more complex compared to other abdominal organs such as kidneys, pancreas or spleen, because the blood inflow of the liver is made up by two components, which include inflow from both the hepatic artery and the portal vein $(4,5)$. These two vessels provide blood to the liver in two different time settings, which are related to each other in the analysis of arrival, distribution and excretion of contrast media. Additionally, the analysis of data may potentially be influenced by different flow curves, which include a typical arterial enhancement curve (diastolic and systolic peaks) for the hepatic artery, and a continuous positive venous inflow curve for the portal vein(6)

In previous studies, several imaging methods for non-invasive quantification of hepatic hemodynamics have been proposed(3). Ultrasound can be applied for quantification by means of Doppler ultrasonography or contrast-enhanced ultrasonography(7-9). However, the main limitation of ultrasound examinations is that it is operator dependent, hence with low reproducibility, and has low sensitivity(10). Computed tomography (CT) has been proposed and evaluated for quantification of enhancement patterns(11). With the introduction of modern multislice multidetector CT scanners, even more robust data can be obtained, allowing for a multitude of postprocessing techniques, but this remains yet to be defined(12). MR imaging is increasingly being used for analysis of liver diseases. The unique ability of MR imaging to detect intrinsic tissue components, in combination with capability of multiphasic dynamic imaging after gadolinium administration render it a superior technique for both the detection and characterization of focal liver lesions, without radiation exposure(10). In addition, in the future, the combination of diffusion-weighted imaging of the liver, MR-elastography and perfusion imaging may provide additional tools to assess the liver function, enabling a combination of both morphologic and metabolic imaging in a one-step imaging protocol(13). MR imaging has been proposed as well for quantification of hepatic perfusion and biliary excretion(14, 15), using different types of contrast agents including T1-shortening agents, T2-shortening agents and macromolecular agents(16).

The purpose of this review article is to explain and illustrate the current status and potential applications of automated and color-coded post processing techniques for analysis of dynamic multiphasic gadolinium-enhanced MR imaging of the liver using state-of-the-art MR imaging exams.

\section{MR imaging technique for dynamic multiphasic imaging}

Breath-hold T1-weighted contrast-enhanced dynamic magnetic resonance imaging of the liver can be performed on any modern MR imaging unit, with a magnetic field strength >1.0 Tesla (T). Because the introduction of parallel imaging has facilitated fast acquisition of sequences, with increased possibilities for dynamic contrast-enhanced imaging of the abdomen, this will be discussed shortly.

Parallel MR imaging (pMRI) is based on a method to undersample K-space in order to reduce total scan time. Currently, pMRI is routinely available in all commercial MR scanners, including sensitivity-encoded imaging (SENSE), array spatial sensitivity encoding technique (ASSET), simultaneous acquisition of spatial harmonics (SMASH) and others $(17,18)$. The undersampling of the k-space data, with some loss of the signal-to-noise ratio (SNR), results in considerably increased temporal resolution which is essential for a better TIC fit. Currently, pMRI is still being improved, and new methods are being published on a regular basis(19-21).

For dynamic contrast-enhanced imaging, scan protocols are tailored for the desired measurements. The choice of sequence and parameters therefore, will depend on anatomic coverage, acquisition times, susceptibility to artifacts resulting from magnetic field inhomogeneities and need for quantification(22). For dynamic multiphasic imaging, 2D/3D T1-weighted sequences (gradient-recalled echo (GRE), saturation recovery / inversion recovery snapshot sequences, or echo-planar sequences) can be performed after administration of an intravenous bolus of $0.1 \mathrm{mmol} / \mathrm{kg}$ 
of a non-liver-specific gadolinium-chelate such as Gd-DTPA (Magnevist, Schering AG, Berlin, Germany) or Gd-DTPABMA (Omniscan, GE Healthcare, Oslo, Norway). To correct additionally for the loss in SNR by the use of pMRI, the total dosage may be slightly increased for compensation. Routinely, an injection rate of $3 \mathrm{ml} / \mathrm{s}$ is used by means of a power injector, followed by a bolus of saline ( 15 or 20cc total). The higher injection rate compensates for the SNR loss due to the pMRI application.

Dynamic imaging of the liver entails imaging in at least four different vascular phases that may be extended depending on performance of the system. The phases that should be included are: 1) precontrast phase, 2) arterial phase, 3) portal (or equilibrium) phase and 4) delayed (or venous) phase). To accurately capture the arterial phase, a timing bolus technique can be applied, using $2 \mathrm{cc}$ of the same contrast agent with inject rate $3 \mathrm{ml} / \mathrm{s}$ bolus followed by $15 \mathrm{cc}$ saline. The timing bolus technique and adjustment of the total amount of contrast according to the body weight will compensate for differences in cardiac output and distribution volume. The portal phase is usually acquired at 45 seconds after the arterial phase, and the delayed phase at least 120 seconds after acquisition of the arterial phase(2325). The sequence should be adjusted to obtain full anatomic coverage of the liver in each scanned phase within a maximum of one breath-hold period ( $<25 \mathrm{sec}$ ). Routinely, a slice thickness of $6-8 \mathrm{~mm}$ is used for 2D GRE sequences, which can be thinner ( 3 or 4mm) in 3D GRE sequences. Ideally, a time-resolved gadolinium-enhanced sequence should be applied to have more data points on the TIC. In the abdomen, however, due to the breath holding requirements it is not always possible yet to have continuous scanning in order to obtain multiple scans within each vascular phase. Therefore, most body MRI centers acquire at least four distinct phases.

With the introduction of higher-field-strength magnets (3.0T), improved dynamic imaging of the abdomen became feasible through inherently increased SNR (theoretically on the order of 2), high performance gradient-systems and advanced software platforms. This has enabled increased spatial resolution, scanning with thinner slices or accelerated scanning times, which is particularly useful for angiographic MR imaging $(26,27)$.

\section{Contrast agents for dynamic multiphasic imaging}

Dynamic multiphasic contrast-enhanced MR imaging may be performed with low- or high-molecular-weight agents, non-liver-specific gadolinium chelates or liver-specific gadolinium chelates. After injection into the intravascular compartment, non-liver-specific gadolinium-chelates such as Magnevist and similar low molecular-weight agents are rapidly diluted in the circulating plasma volume. They rapidly leak out of this space into the extra-vascular (or extra-cellular) space (interstitium) and then equilibrate between the intravascular and interstitial spaces. Only $1-2 \%$ at most of an administered dose enters cells(28). The non-liver-specific gadolinium-chelates that are most frequently used in humans are extracellular agents. In these contrast agents, tissue enhancement depends on arterial input function, kinetic of distribution of blood into the capillary bed, leakage across the capillary walls, and volume of the interstitial space. However, they have inherent disadvantages in estimating blood volume and capillary permeability exclusive of the brain due to the high rate of their vascular extraction, even in normal vessels.

Macromolecular contrast agents, also called blood pool agents, have been developed, such as gadopentetate dimeglumine-labeled albumin(29). Blood pool contrast agents have advantages for both spatial resolution and SNR at vascular imaging. However, the availability as well as the direct clinical applicability of blood pool agents is limited, due to the slow glomerular filtration rate (GFR) and weak and reversible protein-binding.

The development of liver-specific gadolinium-chelates has increased the accuracy of MR for identification and characterization of focal liver lesions(30). Liver-specific gadolinium chelates including gadobenate dimeglumine (Gd-BOPTA) and gadolinium-ethoxybenzyl-diethylenetriaminepentaacetic acid (Gd-EOB-DTPA) with a first phase of extracellular distribution give both dynamic (morphologic) and late phase (functional) information useful for lesion characterization(30). They combine the properties of a conventional extracellular gadolinium-chelate with those of an agent targeted specifically to the liver and behave in a manner analogous to conventional gadolinium-chelates during the dynamic phase of contrast enhancement(31) In the delayed phase it improves the impact of MRI for the detection of liver lesions(30). Some liver-specific contrast agents such as mangafodipir (Teslascan, Mn-DPDP, Amersham, GE Health Care) or superparamagnetic iron-oxide particles (SPIO) have no dynamic imaging capability. The possibility of sequential administration of gadolinium chelates and Teslascan in a single visit to obtain both dynamic and late imaging was proposed(32). However, such combinations are highly undesirable because of the high cost, complex combined T1-shortening effects of two agents, and recently described potential neurotoxic side effects (Parkinson-like progressive extrapyramidal disorder) of Teslascan(33). 


\section{Post-processing hardware and software}

Currently, several vendors provide software packages to facilitate the post processing of gadolinium-enhanced dynamic MRI data on a workstation. For example, Easy Vision (Philips), which runs the Quantitative Analysis package; and 2) Advantage Windows (GE), which supports the Functool package. The Easy Vision package provides the display of a reference image, a subtraction imaging, and nine functional images: relative enhancement, maximum enhancement, maximum relative enhancement, wash-in rate, wash-out rate, brevity-of-enhancement, area-undercurve, time-to-peak, T0. Advantage Windows can display a subtraction imaging, and seven functional images: mean time to enhance, time to maximum enhancement, positive enhancement integral, negative enhancement integral, signal enhancement ratio, maximum slope of increase, and maximum slope of decrease in enhancement. Both software packages allow the display of the time-intensity curves and statistics of region-of-interest (ROI), either as a graph or a table format.

\section{Time intensity curves and derived parameters}

The software package analyses these data over time, to provide a plotted corrected graph which illustrates the step-wise changes in enhancement over time in different vascular phases after contrast administration(34). The pharmaco-kinetic time intensity curves (TIC) model provides a number of different parameters that can be used to characterize specific tumor enhancement patterns. These parameters can be used to obtain data on microvessel density, flow through the vessels, vascular resistance, capillary wall permeability, composition of the extracellular space, and venous outflow. Currently the two most widely applied models for analyzing dynamic MRI Gd-DTPA data are shutter-speed model proposed by Charles S. Springer Jr.and Tofts model proposed by Paul S. Tofts $(35,36)$.

The color-coded images are based on qualitative pixel-wise display of various quantitative modeling parameters, which can be derived from the quantitative data of the TIC at each pixel. This method provides an automatic conversion of kinetic contrast information into easy to interpret color-scaled images, which provides a quick overview of degree and heterogeneity of enhancement within the tissue.

The generation of time intensity curves $(\mathrm{TIC})$ is based on signal intensity measurements obtained by defining region-of-interest areas on the color-coded images, which are placed by the operator. To correct for minimal differences in signal intensity in images acquired before and after injection of the contrast agent, measurements for TIC generation are based on subtracted images only. Subtracting the analyzed contrast-enhanced image with the precontrast image, acquired at the same level, creates these subtractions. This is performed automatically by the software program. It is important to note that semi-automated interpretation of dynamic examination may suffer from respiratory motion, which may potentially cause a varying position of the liver in the z-axis over time. In our experience, this is most frequently encountered in sub-cm focal lesions located under the diaphragm.

An example of a baseline TIC illustrates that a number of parameters can be derived, including specific enhancement patterns such as maximum intensity, maximum enhancement, time to peak, wash-in- and wash-out rate, and brevity of enhancement (Fig. 1). In literature(37), a number of typically shaped TIC have been described (Fig. 2). Type I (steady enhancement) curve shows continuously increasing signal intensity within the lesion, which is considered a benign feature. Type II enhancement curve shows a steep increase in signal intensity in the first phases after contrast administration, which becomes less steep in the more delayed phases, sometimes with plateau formation. This type of curve is considered to be an indicator of viable tumor with stable microcirculation. Type III enhancement curve shows steep arterial enhancement, with loss of signal intensity in the delayed phases, rendering the curve to a negative slope, which is interpreted as washout of contrast within the tumor. This is considered a strong indicator of malignancy, proliferating edge of tumor or vessels.

$\mathrm{TIC}$ is determined by contrast agent in the intravascular and extravascular compartments. During the first pass, the contrast agent is predominantly intravascular and contrast enhancement reflects perfusion and blood volume. Delayed enhancement is determined by the passage of contrast agent into and out of the extravascular space, as determined by rate constants ktrans and kep. 


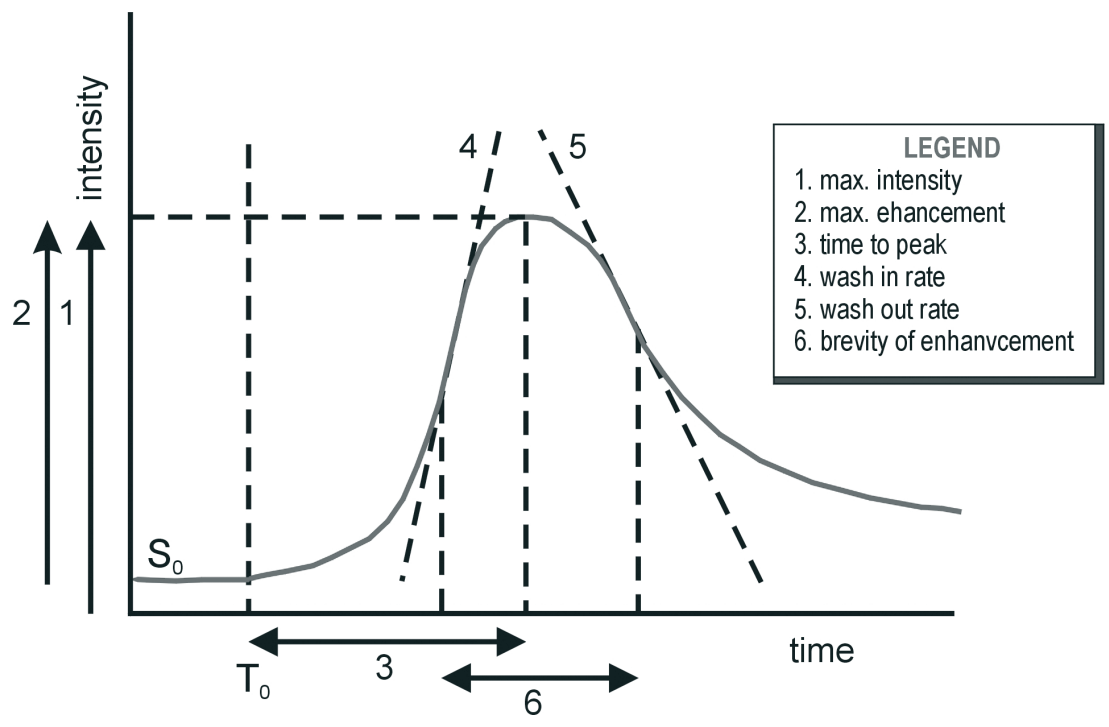

Time intensity curves and color-coded imaging of focal and diffuse liver lesions Benign liver lesions

According to the currently used nomenclature for hepatocellular focal liver lesions, two types of liver lesions exist: regenerative and neoplastic or dysplastic lesions(41). Regenerative lesions include regenerative nodules, segmental or lobar hyperplasia, and focal nodular hyperplasia. Neoplastic or dysplastic lesions include hepatocellular adenoma, dysplastic foci, dysplastic nodules, and hepatocellular carcinoma (HCC).

Figure 2: Typical time-intensity curves (TIC)

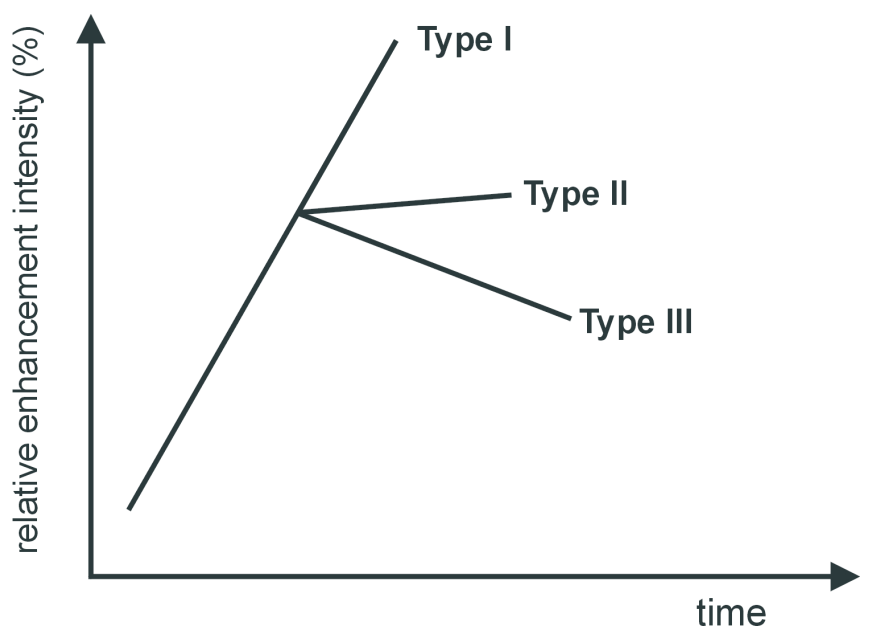

Hepatocellular lesions with benign growth behavior are common, and include cysts, hemangiomas, focal nodular hyperplasia (FNH) and hepatocellular adenomas, although the latter one is referred to as neoplastic lesion because of rare reports of malignant transformation $(23,42)$. To illustrate the role of the subtraction images, and the timeintensity curves in various types of benign lesions, examples of hemangioma, hepatocellular adenoma, and FNH are provided below. 


\section{Definitions of parameters derived from TIC or multicompartment pharmacokinetic modeling:}

TO Time of arrival of contrast inflow, which determines the arrival moment of the contrast agent at the tissue.

So Intensity before arrival of contrast inflow.

1. Maximum intensity: absolute peak value of curve.

2. Maximum enhancement: difference between peak value and SO.

3. Time to peak (uptake speed): time between $\mathrm{TO}$ and time of peak intensity.

4. Wash in rate: maximum slope between T0 and time of peak intensity, which determines the maximum rate of contrast agent uptake during the acquisition and can adequately estimate the degree of early strong enhancement of tumor tissue..

5. Wash out rate: absolute value of maximum slope between time of peak intensity and last measurement point, which determines the maximum rate of contrast agent outflow during the acquisition.

6. Brevity of enhancement: time between wash in and wash out.

7. Relative enhancement: percentage of signal intensity increases between the post-contrast and the pre-contrast signal intensities respectively. Relative enhancement reflects the plasma volume and is highly correlated with microvessel density

8. Maximum relative enhancement: percentage of signal intensity increase between the maximum post contrast and the pre-contrast signal intensities.

9. Area under curve: sum (integral) of the area underneath the time intensity curve.

10. Volume transfer constant (ktrans): the constant determines the passage of contrast agent into the extravascular compartment from the intravascular compartments. ktran approximates to endothelial permeability surface area product (kfp)

11. Rate constant (kep): the constant determines the passage of contrast agent out of the extravascular compartment to the intravascular compartments. kep is highly influenced by the vessel permeability and is highly correlated with microvessel density $(38)$ and relative blood volume $(r B V)(39,40)$.

12. Extracellular extravascular space (Ve): the constant determines leakage volume of fractional interstitial space. Ve is the volume of extravascular extracellular space per unit volume of tissue(38)

Hemangiomas are the most common hepatic neoplasms, with an incidence between 0.4-20\%(43). Hemangiomas are lesions with long T2 values, so they have high signal intensity on T2-weighted images. Also, they maintain their signal intensity on images with longer echo times that illustrates the lesion consists of multiple fluid-filled spaces. The vast majority of hemangiomas show a typical peripheral nodular enhancement pattern with complete fill-in of the entire lesion over time. This enhancement pattern is of a typical benign lesion with a Type I curve (Fig. 3).

Hepatocellular adenoma often occur in young adult women and are associated with the used of birth control pills(42). Liver adenomas vary in signal intensity on T1- and T2- weighted images. Characteristically, liver adenomas show a transient blush in the arterial phase of the dynamic Gadolinium-enhanced images, and fade to isointensity on delayed images (Fig. 4).

Focal nodular hyperplasia (FNH) most commonly occurs in adult female patients, but can also occur in $10-20 \%$ of men(44). FNH is not related to the use of birth control pills and is solitary in $2 / 3$ of cases. FNH is mostly slightly hypointense on T1- and slightly hyperintense to the liver on T1-weighted images, and 10-49\% of the cases show a central scar with high signal on T2-wighted images(44, 45). Typically, FNH shows very intense homogeneous enhancement in the arterial phase, with fading to isointensity in the delayed phase. The central scar is enhanced on delayed images. Even though FNH is a benign liver lesion, typically, this type of lesion does not show a Type I, but rather a Type III enhancement curve. The exact reason for this is not clear, but it is important to notice that in the delayed phase, the enhancement curve of FNH remains higher than the liver, indicating absence of washout. The time-intensity curve for FNH is characterized as showing signs of "arterialization" (steep enhancement) in the first part of the curve and subsequently showing signs of "hepatization" (curve running slightly above but parallel to the curve of the liver), as was described in a recent study assessing the MR imaging findings of FNH (Fig. 5)(45). 
CAHAPTHR
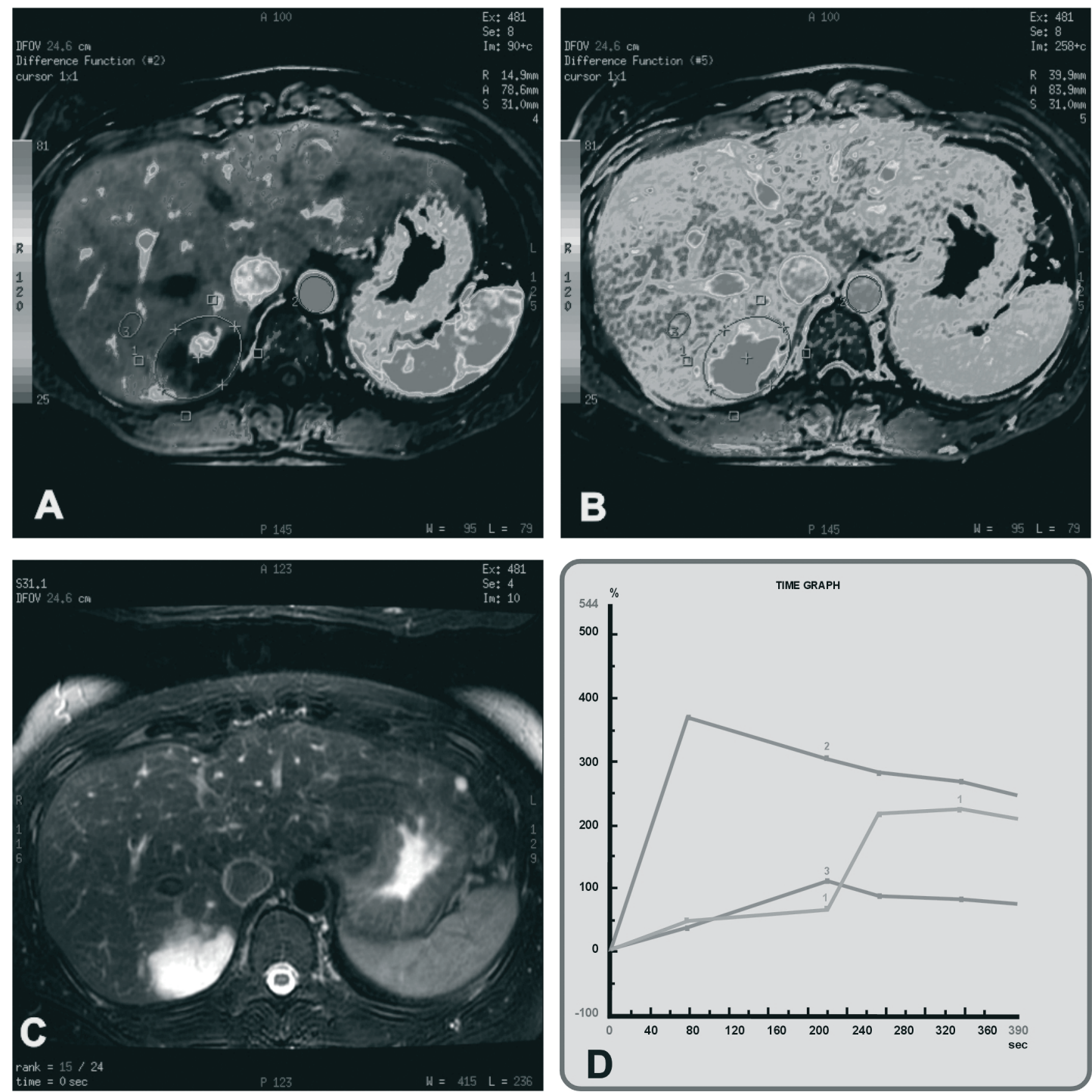

\section{Figure 3: Hemangioma}

40-year-old female with a $48 \times 35 \mathrm{~mm}$ hemangioma in the right lobe of the liver. A) (Color-coded parameter image from the arterial phase, calculated using relative enhancement) Nodular enhancement in the periphery of the lesion. B) (Color-coded parameter image from the delayed phase, calculated using relative enhancement) Complete fill-in of the entire lesion. The lesion is entirely red. C) (T2-weighted image, TR/TE $=1059 / 99.4$ msec) High signal intensity, lobulated and well-circumscribed lesion typical for hemangioma. D) The time-intensity curve from the ROI of the lesion (green curve) reflects the steady enhancement of hemangioma (atypical Type I curve). The time-intensity curve from the ROI of the blue curve (line 3) shows normal liver parenchyma enhancement, and line 2 represents the signal intensity within the aorta at the acquired vascular phase. Note: decremental value in color spectrum: red $>$ orange $>$ yellow $>$ green $>$ blue $>$ indigo $>$ violet.

(Color version available in chapter 18)

\section{Malignant liver lesions}

Malignant liver lesions are common, and include primary liver lesions such as HCC, which develops in a step-wise fashion in patients with cirrhotic liver disease. In this sequence, dysplastic foci may develop into dysplastic nodules, and eventually in HCC. Metastatic liver disease is a major health problem, which occurs most commonly in patients with colorectal malignancies. To illustrate the role of the subtraction images, color-coded images, and the TIC in primary and secondary liver lesions, examples of HCC and liver metastasis are provided below. 

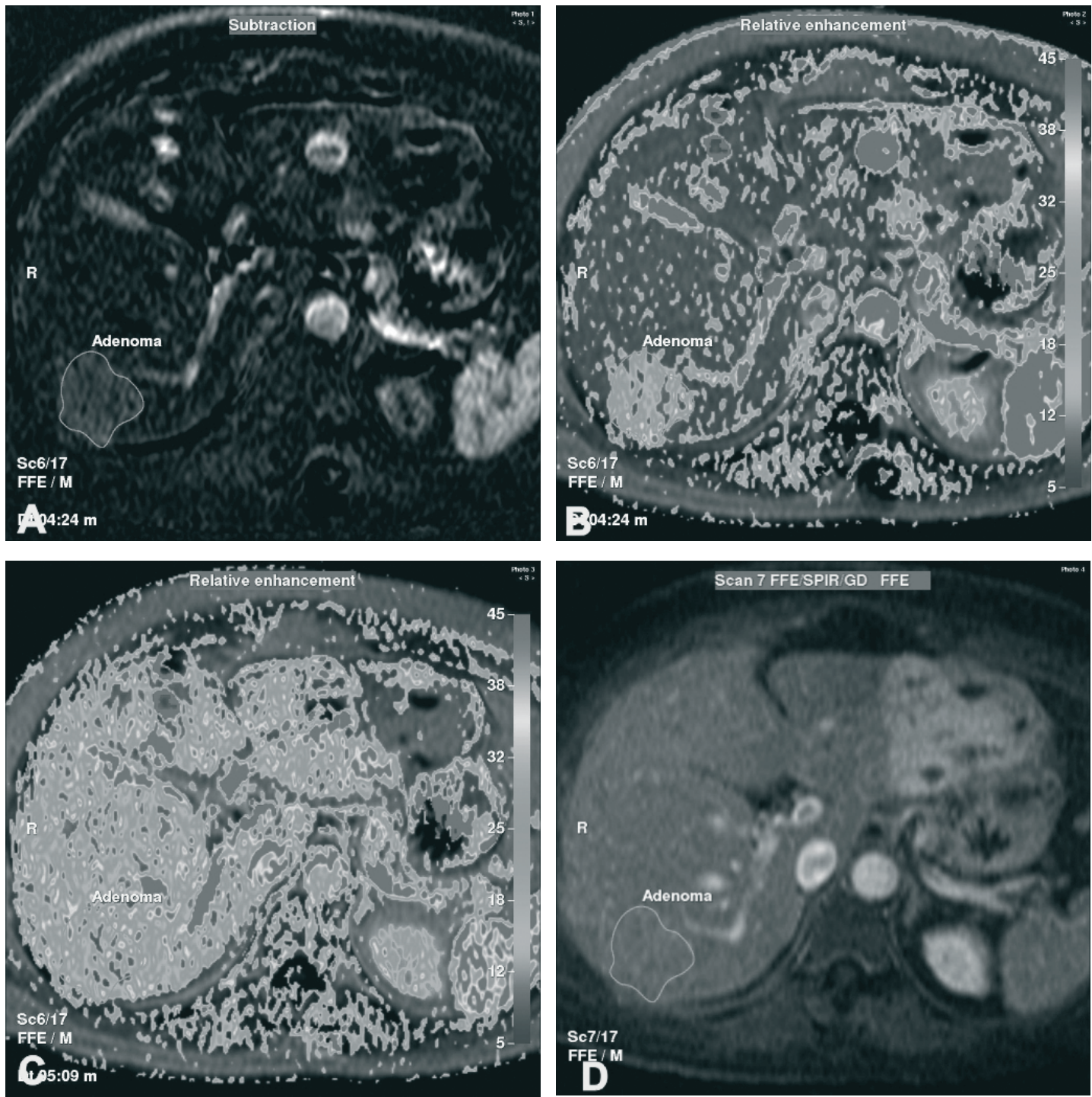

\section{Figure 4: Hepatocellular adenoma}

32-year-old female with a hepatocellular adenoma in the right lobe of liver and a hemangioma in the left lobe of the liver. A) (Subtraction image from the arterial phase) Hypervascular lesion. B) (Color-coded parameter image from the arterial phase, calculated using relative enhancement) Homogeneous blush of the lesion. C) (Color-coded parameter image from the portal phase, calculated using relative enhancement) Isointensity of the lesion with the surrounding liver. D) (Delayed fat-suppressed T1-weighted image) Isointensity of the adenoma in the right lobe of the liver and homogeneously enhanced hemangioma in the left lobe of the liver. Note: decremental value in color spectrum: red > orange > yellow > green $>$ blue > indigo > violet.

(Color version available in chapter 18).

$\mathrm{HCC}$ is the most common primary malignancy of the liver and occurs most frequently in males(1). HCC is solitary in $50 \%$, multifocal in $40 \%$, and diffuse in $10 \%$ of the cases. Often, HCC will show a moderately high signal on T2weighted images. HCC typically shows intense enhancement of the tumor in the arterial phase (high wash-in rate) and looses much of its contrast in later phases (high wash-out rate). In later phases, most HCC's show enhancement of a tumor capsule. A combination of high signal intensity on T2-weighted images and the enhancement pattern described above, particularly in a patient with pre-existing hepatitis or cirrhosis, is considered diagnostic for HCC. HCC's demonstrate Type III enhancement curves, in which the delayed phase images show a lower signal intensity of the lesion compared to the lesion (washout) (Fig. 6-8). Fibrolamellar HCC is an uncommon variety of HCC, which often occurs, in young female patients(23). At MRI, fibrolamellar HCC may show some overlap with FNH, including 

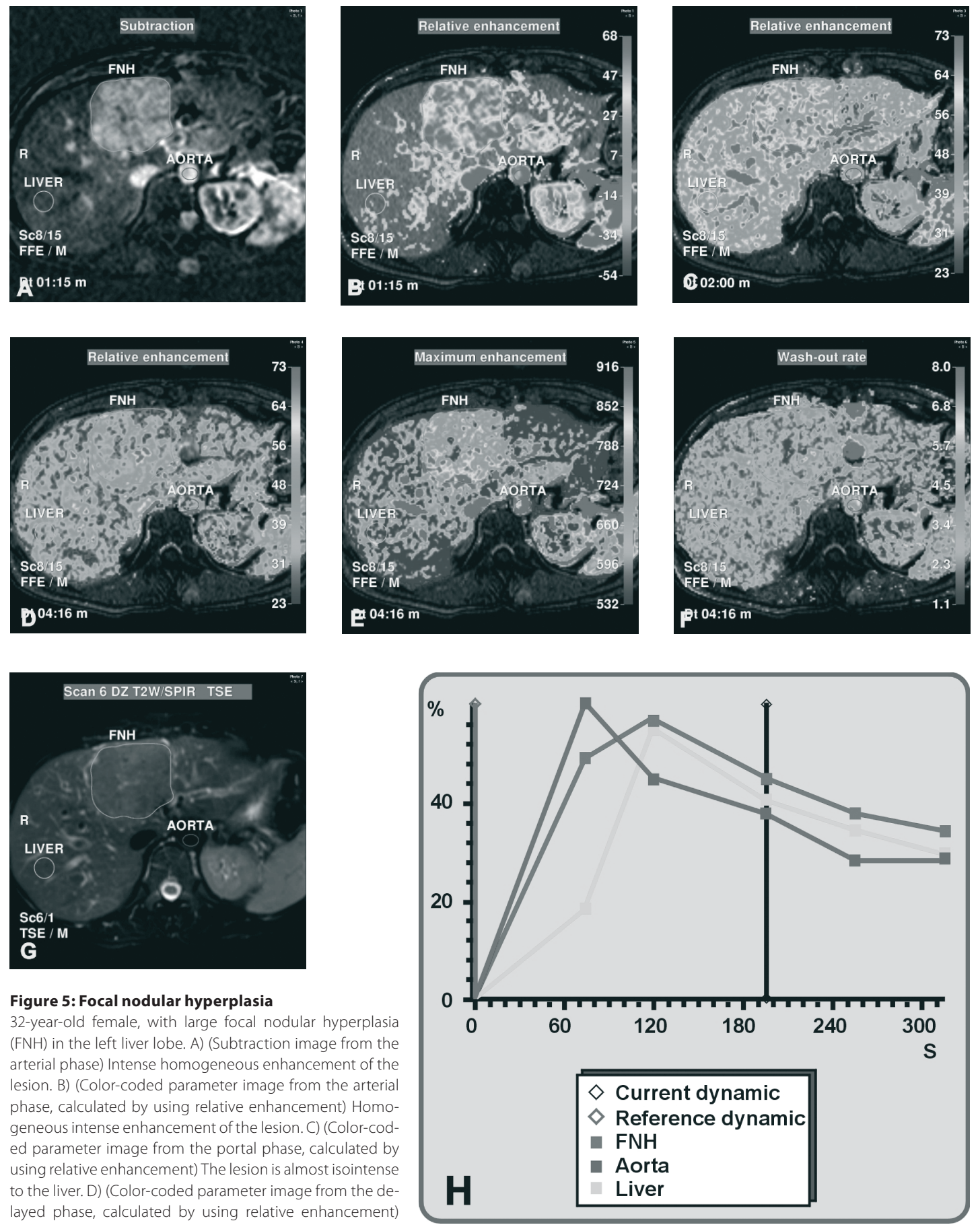

Figure 5: Focal nodular hyperplasia

32-year-old female, with large focal nodular hyperplasia $(\mathrm{FNH})$ in the left liver lobe. A) (Subtraction image from the arterial phase) Intense homogeneous enhancement of the lesion. B) (Color-coded parameter image from the arterial phase, calculated by using relative enhancement) Homogeneous intense enhancement of the lesion. C) (Color-coded parameter image from the portal phase, calculated by using relative enhancement) The lesion is almost isointense to the liver. D) (Color-coded parameter image from the delayed phase, calculated by using relative enhancement) Delayed enhancement of the central scar. E) (Color-coded parameter image, calculated by using maximum enhancement) The absolute values of enhancement of the lesions are higher than the liver. F) (Color-coded parameter image, calculated by using wash-out rate) This lesion has a similar wash-out rate as the surrounding liver. G) (T2- weighted image ( $T R / T E=1500 / 80 \mathrm{msec}$ ) The lesion is only slightly hyperintense to the liver. H) The time-intensity curve of the lesion (green curve) parallels the curve of the aorta in the arterial phase, indicating a very steep enhancement (Type III). In the delayed phases it parallels the curve of the liver, reflecting its benignity as well as its similarity to the normal liver tissue. Note: decremental value in color spectrum: red $>$ orange $>$ yellow $>$ green $>$ blue $>$ indigo $>$ violet.

(Color version available in chapter 18). 

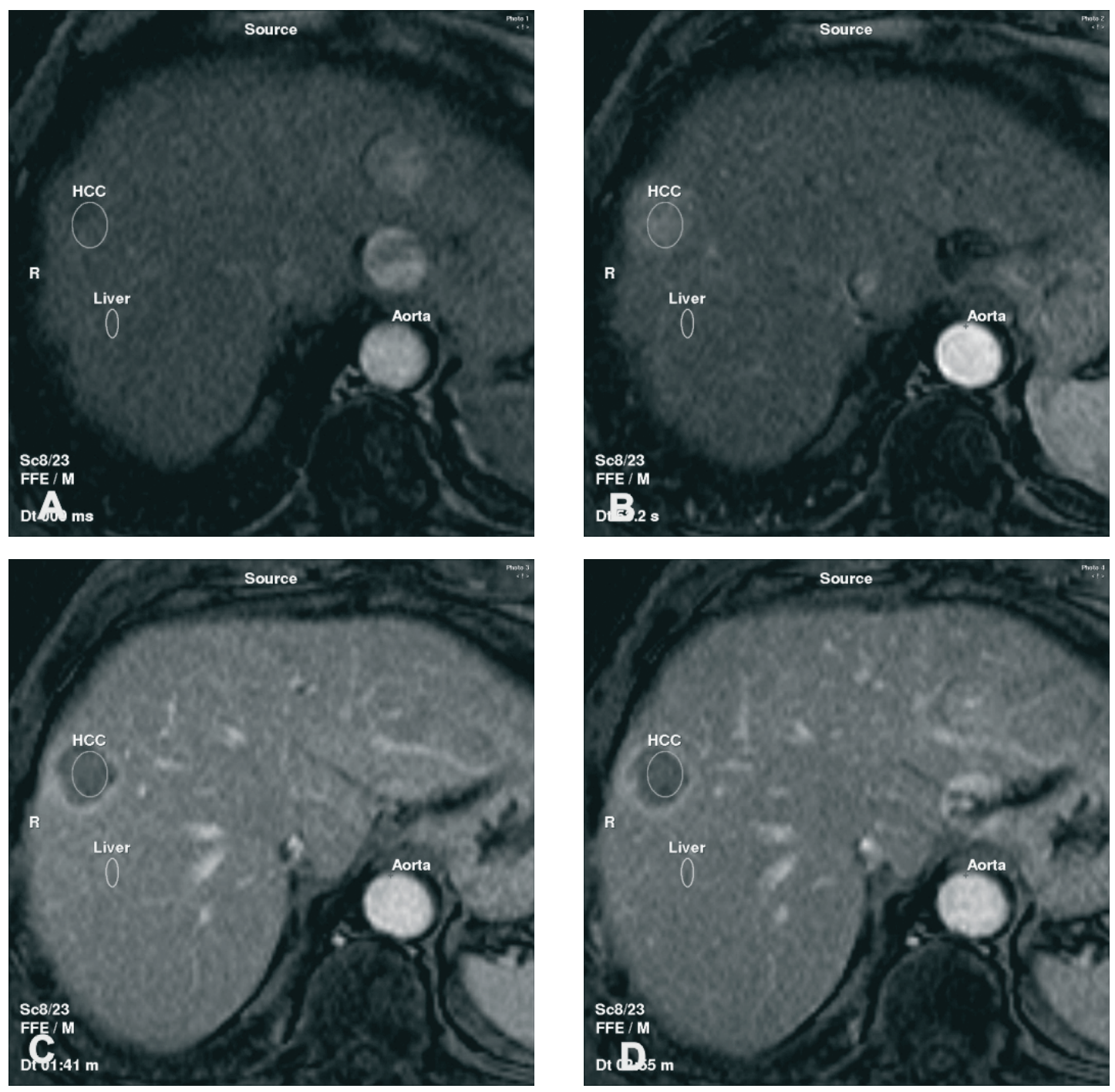

\section{Figure 6: Hepatocellular carcinoma}

62-year-old male with hepatocellular carcinoma (HCC) in the right liver lobe. A-D) Four images illustrating the typical four phases of dynamic gadolinium enhanced sequence, including the pre-contrast phase (A), arterial phase (B), portal phase (C), and delayed phase (D). Note that the HCC is intensely enhancing in the arterial phase and shows wash-out in the delayed phases with late enhancement of a pseudocapsule. (Color version available in chapter 18).

arterial enhancement and presence of a fibrous central scar. Important differences between FNH and fibrolamellar HCC include: 1) a more pronounced and homogeneous enhancement in the arterial phase for $\mathrm{FNH}$, and heterogeneous, less pronounced arterial enhancement for fibrolamellar HCC; and 2) presence of washout in the delayed phase in fibrolamellar HCC and persistent higher signal intensity in the delayed phase after contrast administration in FNH. Time-intensity curves as well as the color-coded images may further facilitate the distinction between fibrolamellar HCC and FNH.

Liver metastases are the most common malignant tumors of the liver. Optimal evaluation with MR imaging includes detection as well as characterization. Characterization is important because patients with known primary malignancies commonly have small hepatic lesions that are benign cysts or hemangiomas. In addition, accurate assessment of the segmental extent of the metastatic disease may have a substantial impact on patient management. Liver metastases are generally low on T1-and moderately high on T2-weighted images. 
Small hypervascular metastases may show intense enhancement in the arterial phase. Most commonly, liver metastases show a peripheral irregular ring of enhancement. The color-coded images may provide more information concerning the vascularity of the lesions. The time-intensity curves of liver metastases most closely fit into the Type II enhancement curves. The enhancement pattern is similar to the surrounding liver in the arterial phase, but with different behavior in the later phases, including the portal and delayed phases. In these phases, the intensity of enhancement is routinely lower than the surrounding liver, resulting in a Type II enhancement curve which is located below the enhancement curve of the surrounding hepatic stroma (Fig. 9).

\section{Diffuse liver lesions}

Diffuse liver parenchymal abnormalities may include a wide variety of disease entities, such as hepatitis, cirrhosis, hemochromatosis, steatosis, and diffuse malignancies $(1,23)$. Acute hepatitis may present with reversible
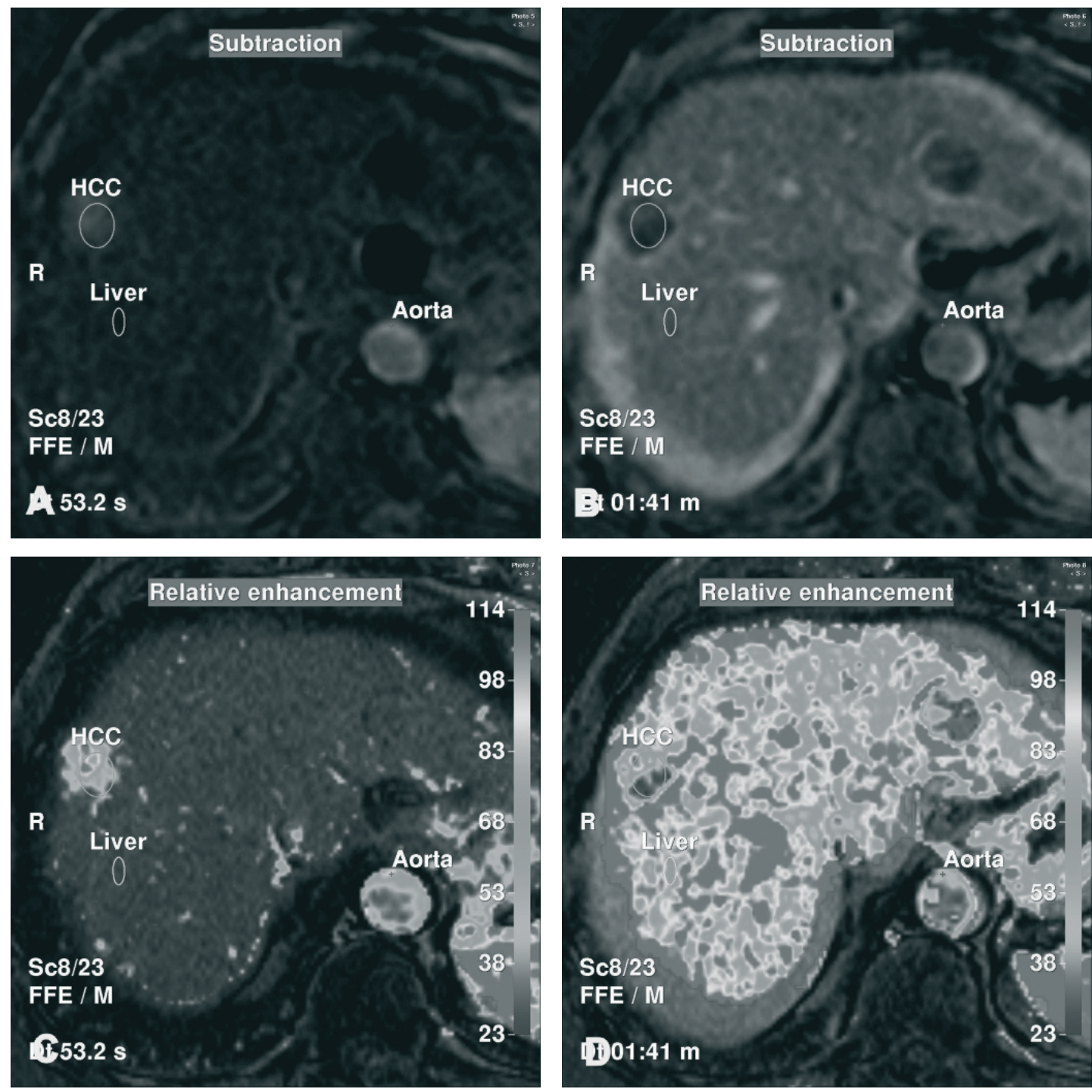

\section{Figure 7: Hepatocellular carcinoma}

The same patient as in Figure 6. A-B) (Two subtraction images from the arterial and portal phases, respectively) Improved visualization of the HCC. C-D) (Two color-coded parameter images from the arterial and portal phases, respectively, calculated by using relative enhancement) Heterogeneous enhancement of the lesion, i.e. the lesion is enhancing more intensely in the center than in the periphery (more red in the center) (C), and lost contrast in the center (sign of a high wash-out rate), with enhancing pseudocapsule (more red in the periphery of the lesion) (D).

(Color version available in chapter 18). 

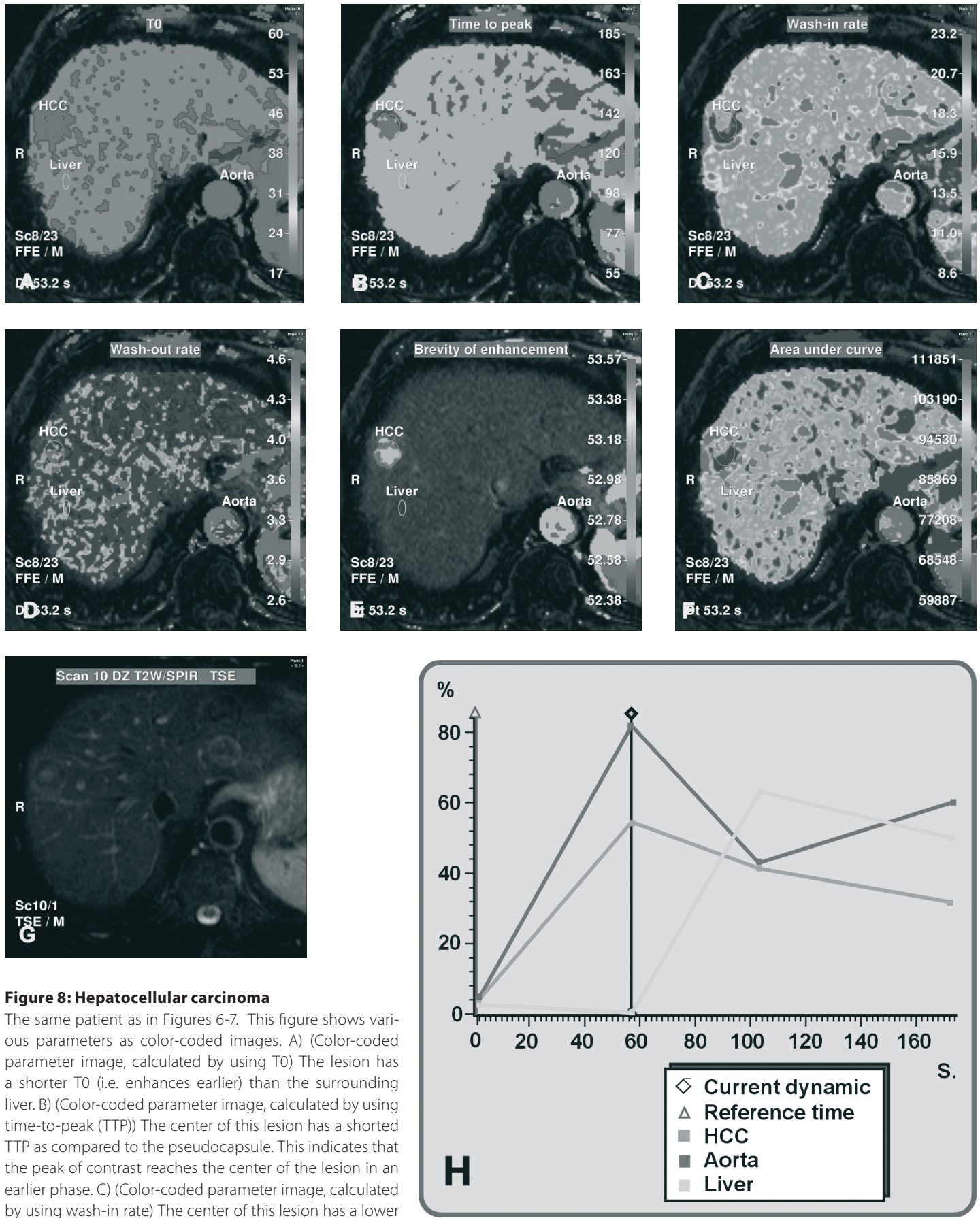

\section{Figure 8: Hepatocellular carcinoma}

The same patient as in Figures 6-7. This figure shows various parameters as color-coded images. A) (Color-coded parameter image, calculated by using TO) The lesion has a shorter TO (i.e. enhances earlier) than the surrounding liver. B) (Color-coded parameter image, calculated by using time-to-peak (TTP)) The center of this lesion has a shorted TTP as compared to the pseudocapsule. This indicates that the peak of contrast reaches the center of the lesion in an earlier phase. C) (Color-coded parameter image, calculated by using wash-in rate) The center of this lesion has a lower wash-in rate as compared to the pseudocapsule. D) (Color-

coded parameter imaging, calculated by using wash-out rate) The center of the lesion has a slightly higher wash-out rate than the surrounding liver. E) (Color-coded parameter image, calculated by using brevity-of-enhancement) The time between the wash-in rate and wash-out rate is longer in the center of this lesion as compared to the surrounding liver. F) (Color-coded parameter image, calculated by using area-under-curve) The pseudocapsule of this lesion received a higher dosage of contrast agent than the center of the lesion. G) (T2-weighted image (TR/TE=1500/80 msec) Slightly hyperintensity in the center as well as the pseudocapsule of the lesion. H) The time-intensity curve shows that the lesion (green curve) enhances more than the liver and less than aorta in the arterial phase, and loses contrast in the later phases (sign of wash-out). Please note that the curve, unlike the curve in the FNH (see Figure $5 \mathrm{H}$ ), is not parallel to the curve of the liver in any phase. Note: decremental value in color spectrum: red $>$ orange $>$ yellow $>$ green $>$ blue $>$ indigo $>$ violet. (Color version available in chapter 18). 

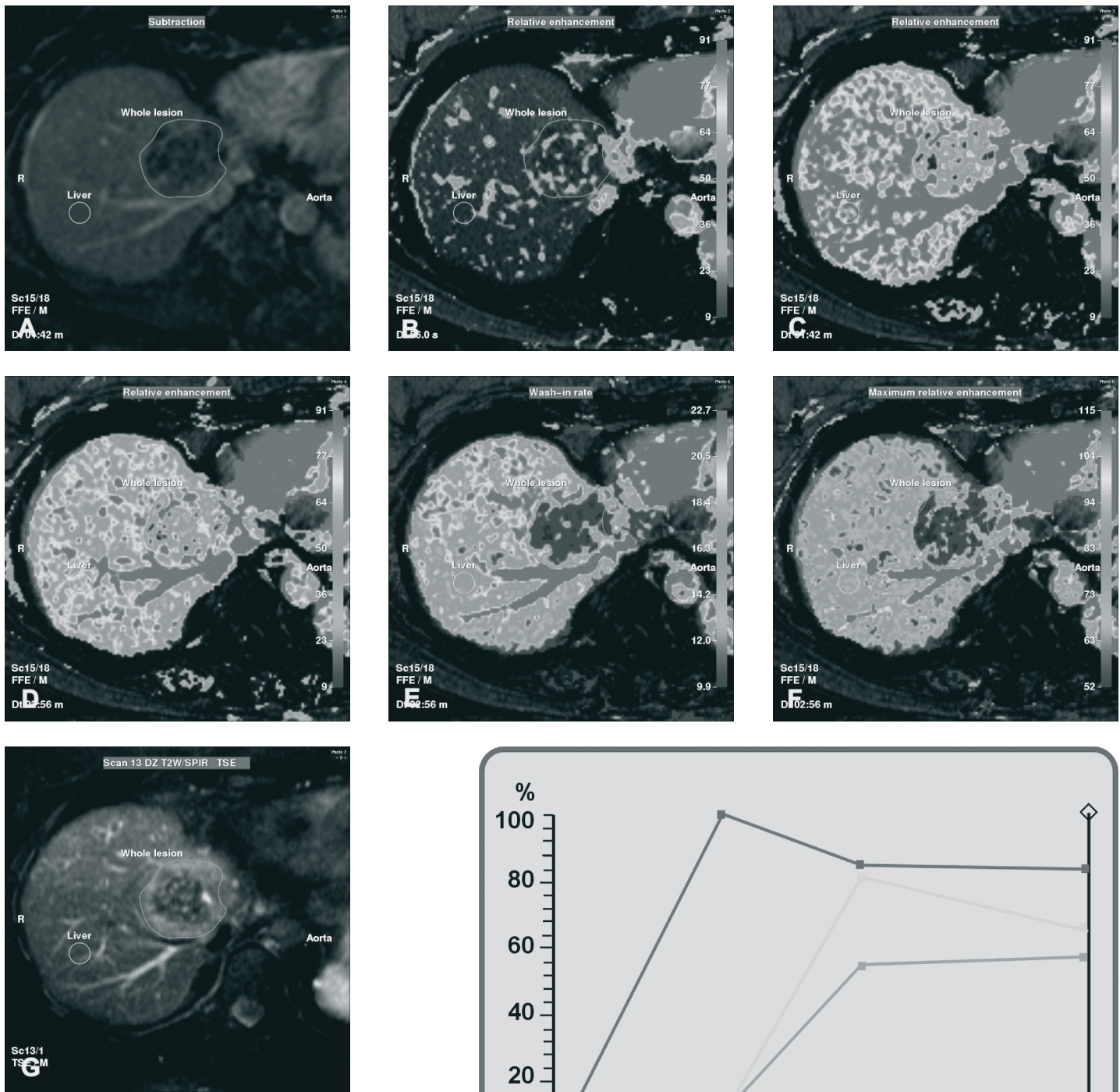

Figure 9: Liver metastasis

56-year-old female with a large hepatic metastasis from sigmoid carcinoma. A) (Substraction image from the portal phase) Irregular ring enhancement of the lesion as well as a faint enhancement in the center of the lesion. B) (Colorcoded parameter image from the arterial phase, calculated by using relative enhancement) Faint enhancement of the lesion. C) (Color-coded parameter image from the portal phase, calculated by using relative enhancement) Irregular ring of enhancement in the periphery of the lesion, whereas the more central parts of the lesion are enhanced less

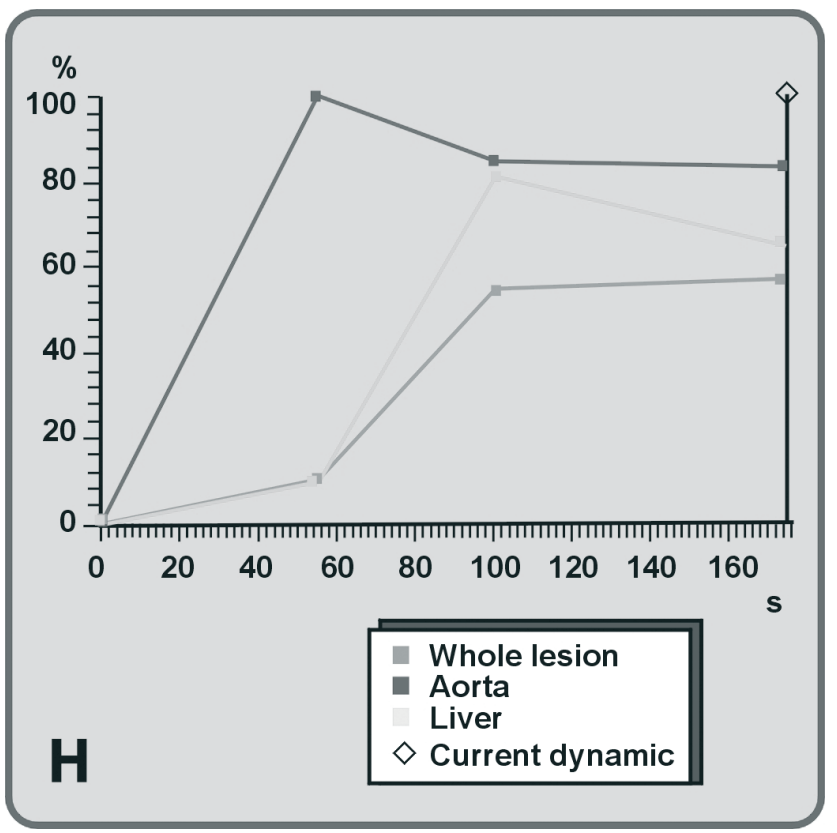
than the surrounding liver. D) (Color-coded parameter im-

age from the delayed phase, calculated by using relative enhancement) The lesion has enhanced slightly more than in the previous phase, whereas the liver has lost some of its contrast. Therefore, the conspicuity of lesion has decreased. E) (Color-coded parameter image, calculated by using wash-in rate) The periphery of the lesion has a higher wash-in rate than the center of the lesion. F) (Color-coded parameter image, calculated by using the maximum relative enhancement) The central parts of the lesion enhance less than the surrounding liver. G) (T2- weighted image (TR/ $T E=1500 / 80 \mathrm{msec}$ ) The periphery of the lesion is slightly hyperintense to the liver. H) The time-intensity curve of the lesion (green curve) shows that the lesion is enhancing similar to the liver in the arterial phase. In the portal phase, there is the greatest difference in enhancement between the lesion and the surrounding liver. In the delayed phase, the lesion shows slight further increase in enhancement, whereas, the liver loss some contrast. Therefore, the conspicuity of the lesion decreases after the portal phase. Note: decremental value in color spectrum: red > orange > yellow $>$ green $>$ blue $>$ indigo $>$ violet. (Color version available in chapter 18). 

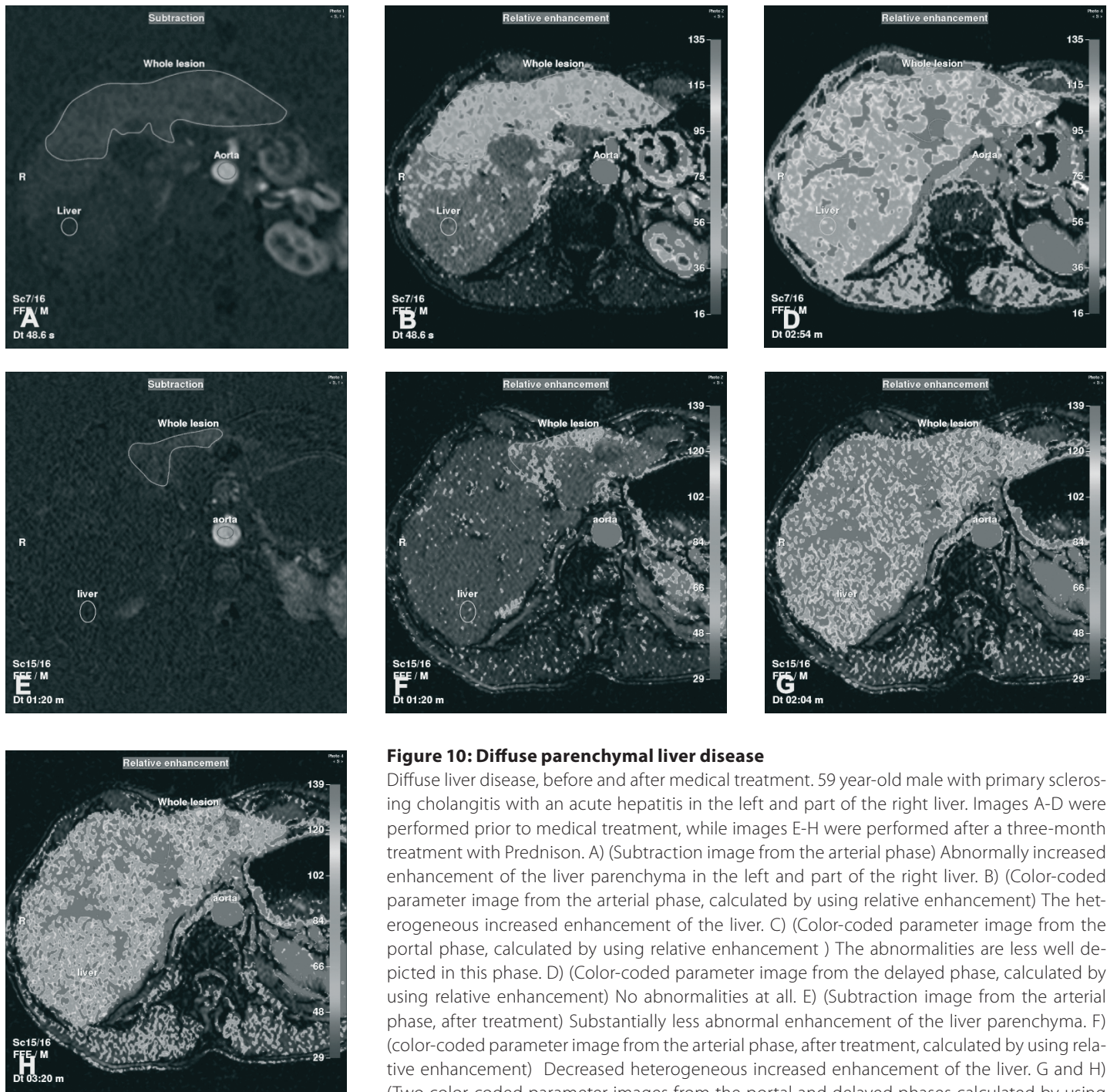

\section{Figure 10: Diffuse parenchymal liver disease}

Diffuse liver disease, before and after medical treatment. 59 year-old male with primary sclerosing cholangitis with an acute hepatitis in the left and part of the right liver. Images A-D were performed prior to medical treatment, while images $\mathrm{E}-\mathrm{H}$ were performed after a three-month treatment with Prednison. A) (Subtraction image from the arterial phase) Abnormally increased enhancement of the liver parenchyma in the left and part of the right liver. B) (Color-coded parameter image from the arterial phase, calculated by using relative enhancement) The heterogeneous increased enhancement of the liver. C) (Color-coded parameter image from the portal phase, calculated by using relative enhancement ) The abnormalities are less well depicted in this phase. D) (Color-coded parameter image from the delayed phase, calculated by using relative enhancement) No abnormalities at all. E) (Subtraction image from the arterial phase, after treatment) Substantially less abnormal enhancement of the liver parenchyma. F) (color-coded parameter image from the arterial phase, after treatment, calculated by using relative enhancement) Decreased heterogeneous increased enhancement of the liver. $\mathrm{G}$ and $\mathrm{H}$ ) (Two color-coded parameter images from the portal and delayed phases calculated by using relative enhancement) The abnormalities have become occult. Note: decremental value in color spectrum: red > orange $>$ yellow $>$ green $>$ blue $>$ indigo $>$ violet.

(Color version available in chapter 18).

heterogeneous patchy enhancement of the hepatic stroma in the arterial phase. In the portal and delayed phases, the abnormal enhancement pattern is occult. To illustrate the role of the subtraction images and the color-coded images in detection of diffuse liver parenchymal disease as well as monitoring of therapy, an example of acute hepatitis in a patient with primary sclerosing cholangitis before and after medical treatment with Prednison is provided (Fig 10).

\section{Discussion}

Post-processing of dynamic gadolinium-enhanced MRI exams of the abdomen allows generation of the colorcoded images and time-intensity curves(46). The information may further improve the diagnostic capability of MRI at high temporal and spatial resolution. Unlike the original gadolinium-enhanced images, the color-coded images provide information concerning the intra-tumoral distribution of vessels (vascular density) as well as the nature of the vascular wall (intact or immature and leaky blood vessels). This may contribute to a better understanding of 
tumor enhancement patterns and angiogenesis, and hence may result in improved characterization of tumors. As described in literature(47), tumors do not grow beyond $2 \mathrm{~mm} 3$ in size as a result of hypoxia unless angiogenesis, a process of the development of new capillaries derived from host tissues allow tumors to grow exponentially.

\section{The enhancement mechanisms behind dynamic gadolinium-enhanced MRI}

Because of the unique dual blood supply of the liver (arterial and portal), hepatic lesions probably have different contrast kinetics as compared to lesions in other organs with only a single (arterial) blood supply. Therefore, the generated TICs of focal liver lesions should always be compared with the curves of the surrounding liver.

The mechanisms behind the differential contrast enhancement are thought to include differences in tumor perfusion and the levels of tumor capillary wall permeability and hydrostatic pressure. The growth of a tumor, and its associated angiogenesis, is often rapid and disordered, leading to: 1) a relatively high fraction of immature blood vessels; 2) abnormal basal membranes in the vessel wall with relative lack of pericytes or smooth muscle associations with endothelial cells which render the vessels more "leaky" or permeable (providing an access point for contrast agents); 3) tumor vessels are tortuous, vary in diameter, and tend toward excessive branching and shunt formation; 4) intermittent or unstable blood flow with acutely collapsing vessels; and 5) hydrostatic pressure differences between tumors and normal tissue, partly reduced or absent lymphatics which may influence contrast agent uptake.

Blood volume, blood flow, mean transit time and microvascular permeability-surface area product are all, in principle, quantifiable through analysis of dynamic gadolinium-enhanced MRI by using pharmaco-kinetic models(36). Tumor regions with dense neovasculature may enhance more rapidly compared to other regions. The position of these regions is related to the distribution of viable blood supply within the tumor. In necrotic or cystic regions, vascular density can be extremely low, whereas in highly active regions, the vascular density can be high.

\section{Dynamic contrast-enhanced MR imaging proposed as an in-vivo marker of angiogenesis}

Due to its high quality and reproducible measurements, dynamic contrast-enhanced MR imaging is being proposed as an in-vivo marker of angiogenesis $(22,48)$ with the advantage over the "gold standard" of direct histologic quantification of angiogenesis with discrepancy between "true" functional and histologic microvessel density: 20\% to $85 \%$ of microvessels are perfused at any given time depending on the microenvironment(22, 39). Dynamic contrastenhanced MR imaging may help define the pharmacological response and dose of vascular endothelial growth factor (VEGF)-mediated angiogenesis inhibitors, such as PTK787/ZK 222584, an orally active inhibitor of VFGF receptor tyrosine kinases, for further clinical development in patients with advanced colorectal cancer(49).

\section{Functional CT versus dynamic contrast-enhanced MRI}

Functional CT using multidetector row, as with dynamic contrast-enhanced MRI, has also been proposed for evaluation of tumor vascularity. Both techniques are easily incorporated into routine examinations and provide reliable quantitative perfusion data, although for both imaging modalities, there remains a lack of consensus regarding optimal acquisition technique, type of analysis method, and surrogate measurement to use(22). There are differences between the two imaging modalities in acquisition techniques, mathematical analysis, measurement parameters, and propensity to artifacts(22). The benefit of functional CT is the direct linear relationship between CT attenuation value and contrast agent concentration. The functional $\mathrm{CT}$ analysis technique includes deconvolution, distributed parameter model. Unfortunately, the risks of contrast agent reactions and potential biologic hazards from radiation exposure to the patients (especially oncologic patients that undergo repeated scans to monitor therapy response) are associated with functional $C T$. The radiation burden associated with a functional CT acquisition protocol is determined by the number of images in the sequence and the tube current (mAs) used for each image. Dynamic contrast-enhanced MRI does not involve the use of ionizing radiation, which is a particular advantage for serial examinations to monitor treatment response. The choice between functional CT and dynamic contrast-enhanced MRI depends on machine availability, doctor expertise, tumor location, desired parameters, and the requirement of decreased radiation burden(22).

\section{The benefits of the color-coded images and time-intensity curves}

Color-coded images can provide information concerning focal as well as diffuse disease processes. This reduces the risk of creating regions-of -interest ( $\mathrm{ROI}$ ) containing more than one tissue type, and makes further $\mathrm{ROI}$ analysis more accurate. In addition, the color-coded images combine both the enhancement pharmaco-kinetics of tissues and morphology, and visualize the enhancement changes over time. 
The generation of time-intensity curves by placing regions-of-interest (ROI) has two benefits over functional images: 1) sampling only a limited number of pixels at once increases the signal-to-noise ratio; and 2) small spatial misregistration between points only introduce small errors. However, there are also some drawbacks. The time-intensity curves are insensitive to regional variation in the parameter being examined, small foci of high or low signal intensity may be missed as they are averaged out within an ROI, and diffuse disease may be difficult to analyze. Patient motion might lead to faulty time-signal intensity curves at each pixel, and hence to faulty functional images.

The parameters derived from the time-intensity curves, which is the basis of the color-coded images have contributions from MR imaging protocols. Therefore, it may be difficult to compare the results of studies from different institutions. Application of pharmaco-kinetic modeling has the potential to standardize these results(22).

\section{ConcLusion}

In conclusion, the generation of color-coded images and time-intensity curves based on dynamic multiphasic gadolinium-enhanced MR imaging data sets provides new opportunities for research. Moreover, it facilitates the diagnostic work-up of hepatic disease for the detection, staging, therapeutic monitoring; and development of antitumor drugs.

\section{RefERENCES}

1. Hussain SM, Semelka RC. Liver masses. Magn Reson Imaging Clin N Am 2005; 13:255-275.

2. Mendez Romero A, Wunderink W, Hussain SM, et al. Stereotactic body radiation therapy for primary and metastatic liver tumors: A single institution phase i-ii study. Acta Oncol 2006; 45:831-837.

3. Materne R, Smith AM, Peeters F, et al. Assessment of hepatic perfusion parameters with dynamic MRI. Magn Reson Med 2002; 47:135142.

4. Becker GA, Muller-Schauenburg W, Spilker ME, Machulla HJ, Piert M. A priori identifiability of a one-compartment model with two input functions for liver blood flow measurements. Phys Med Biol 2005; 50:1393-1404.

5. Materne R, Van Beers BE, Smith AM, et al. Non-invasive quantification of liver perfusion with dynamic computed tomography and a dualinput one-compartmental model. Clin Sci (Lond) 2000; 99:517-525.

6. Cosar S, Oktar SO, Cosar B, Yucel C, Ozdemir H. Doppler and gray-scale ultrasound evaluation of morphological and hemodynamic changes in liver vascualture in alcoholic patients. Eur J Radiol 2005; 54:393-399.

7. Li J, Dong BW, Yu XL, Li CF. Gray scale contrast enhancement and quantification in different positions of rabbit liver. J Ultrasound Med 2006; 25:7-14

8. Renault G, Tranquart F, Perlbarg V, Bleuzen A, Herment A, Frouin F. A posteriori respiratory gating in contrast ultrasound for assessment of hepatic perfusion. Phys Med Biol 2005; 50:4465-4480.

9. Krix M, Plathow C, Kiessling F, et al. Quantification of perfusion of liver tissue and metastases using a multivessel model for replenishment kinetics of ultrasound contrast agents. Ultrasound Med Biol 2004; 30:1355-1363.

10. Hussain SM, Semelka RC. Hepatic imaging: comparison of modalities. Radiol Clin North Am 2005; 43:929-947, ix.

11. Bader TR, Herneth AM, Blaicher W, et al. Hepatic perfusion after liver transplantation: noninvasive measurement with dynamic singlesection CT. Radiology 1998; 209:129-134

12. Pandharipande PV, Krinsky GA, Rusinek H, Lee VS. Perfusion imaging of the liver: current challenges and future goals. Radiology 2005; 234:661-673.

13. Hyslop WB, Balci NC, Semelka RC. Future horizons in MR imaging. Magn Reson Imaging Clin N Am 2005; 13:211-224

14. Planchamp C, Pastor CM, Balant L, Becker CD, Terrier F, Gex-Fabry M. Quantification of Gd-BOPTA uptake and biliary excretion from dynamic magnetic resonance imaging in rat livers: model validation with 153Gd-BOPTA. Invest Radiol 2005; 40:705-714.

15. Schwickert HC, Roberts TP, Shames DM, et al. Quantification of liver blood volume: comparison of ultra short TI inversion recovery echo planar imaging (ULSTIR-EPI), with dynamic 3D-gradient recalled echo imaging. Magn Reson Med 1995; 34:845-852.

16. Wang YX, Hussain SM, Krestin GP. Superparamagnetic iron oxide contrast agents: physicochemical characteristics and applications in MR imaging. Eur Radiol 2001; 11:2319-2331.

17. Pruessmann KP, Weiger M, Scheidegger MB, Boesiger P. SENSE: sensitivity encoding for fast MRI. Magn Reson Med 1999; 42:952-962.

18. Sodickson DK, Manning WJ. Simultaneous acquisition of spatial harmonics (SMASH): fast imaging with radiofrequency coil arrays. Magn Reson Med 1997; 38:591-603

19. Wiesinger F, Van de Moortele PF, Adriany G, De Zanche N, Ugurbil K, Pruessmann KP. Potential and feasibility of parallel MRI at high field. NMR Biomed 2006; 19:368-378.

20. Weiger M, Boesiger P, Hilfiker PR, Weishaupt D, Pruessmann KP. Sensitivity encoding as a means of enhancing the SNR efficiency in steadystate MRI. Magn Reson Med 2005: 53:177-185.

21. Heidemann RM, Griswold MA, Seiberlich N, et al. Direct parallel image reconstructions for spiral trajectories using GRAPPA. Magn Reson Med 2006; 56:317-326.

22. Goh V, Padhani AR. Imaging tumor angiogenesis: functional assessment using MDCT or MRI? Abdom Imaging 2006; 31:194-199.

23. Hussain SM, Semelka RC, Mitchell DG. MR imaging of hepatocellular carcinoma. Magn Reson Imaging Clin N Am 2002; 10:31-52, v.

24. Grazioli L, Morana G, Kirchin MA, Schneider G. Accurate differentiation of focal nodular hyperplasia from hepatic adenoma at gadobenate dimeglumine-enhanced MR imaging: prospective study. Radiology 2005; 236:166-177. 
25. Kim SK, Lim JH, Lee WJ, et al. Detection of hepatocellular carcinoma: comparison of dynamic three-phase computed tomography images and four-phase computed tomography images using multidetector row helical computed tomography. J Comput Assist Tomogr 2002; 26:691-698.

26. Hussain SM, Wielopolski PA, Martin DR. Abdominal magnetic resonance imaging at 3.0 T: problem or a promise for the future? Top Magn Reson Imaging 2005; 16:325-335.

27. Michaely HJ, Dietrich O, Nael K, Weckbach S, Reiser MF, Schoenberg SO. MRA of abdominal vessels: technical advances. Eur Radiol 2006; 16:1637-1650.

28. Dawson P. Functional imaging in CT. Eur J Radiol 2006.

29. Daldrup-Link HE, Shames DM, Wendland M, et al. Comparison of Gadomer-17 and gadopentetate dimeglumine for differentiation of benign from malignant breast tumors with MR imaging. Acad Radiol 2000; 7:934-944.

30. Morana G, Grazioli L, Testoni M, Caccia P, Procacci C. Contrast agents for hepatic magnetic resonance imaging. Top Magn Reson Imaging 2002; 13:117-150.

31. Grazioli L, Morana G, Caudana R, et al. Hepatocellular carcinoma: correlation between gadobenate dimeglumine-enhanced MRI and pathologic findings. Invest Radiol 2000; 35:25-34.

32. Martin DR, Semelka RC, Chung JJ, Balci NC, Wilber K. Sequential use of gadolinium chelate and mangafodipir trisodium for the assessment of focal liver lesions: initial observations. Magn Reson Imaging 2000; 18:955-963.

33. Crossgrove J, Zheng W. Manganese toxicity upon overexposure. NMR Biomed 2004; 17:544-553.

34. Torheim G, Lombardi M, Rinck PA. An independent software system for the analysis of dynamic MR images. Acta Radiol 1997; 38:165172.

35. Li X, Rooney WD, Springer CS, Jr. A unified magnetic resonance imaging pharmacokinetic theory: intravascular and extracellular contrast reagents. Magn Reson Med 2005; 54:1351-1359.

36. Tofts PS. Modeling tracer kinetics in dynamic Gd-DTPA MR imaging. J Magn Reson Imaging 1997; 7:91-101.

37. Kuhl CK, Mielcareck P, Klaschik S, et al. Dynamic breast MR imaging: are signal intensity time course data useful for differential diagnosis of enhancing lesions? Radiology 1999; 211:101-110.

38. Kiessling F, Krix M, Heilmann M, et al. Comparing dynamic parameters of tumor vascularization in nude mice revealed by magnetic resonance imaging and contrast-enhanced intermittent power Doppler sonography. Invest Radiol 2003; 38:516-524.

39. Jackson A, Haroon H, Zhu XP, Li KL, Thacker NA, Jayson G. Breath-hold perfusion and permeability mapping of hepatic malignancies using magnetic resonance imaging and a first-pass leakage profile model. NMR Biomed 2002; 15:164-173.

40. Preda A, van Vliet M, Krestin GP, Brasch RC, van Dijke CF. Magnetic resonance macromolecular agents for monitoring tumor microvessels and angiogenesis inhibition. Invest Radiol 2006; 41:325-331.

41. Terminology of nodular hepatocellular lesions. International Working Party. Hepatology 1995; 22:983-993.

42. Hussain SM, van den Bos IC, Dwarkasing RS, Kuiper JW, den Hollander J. Hepatocellular adenoma: findings at state-of-the-art magnetic resonance imaging, ultrasound, computed tomography and pathologic analysis. Eur Radiol 2006.

43. Terkivatan T, Hussain SM, De Man RA, Ijzermans JN. Diagnosis and treatment of benign focal liver lesions. Scand J Gastroenterol Suppl 2006:102-115.

44. Hussain SM, Terkivatan T, Zondervan PE, et al. Focal nodular hyperplasia: findings at state-of-the-art MR imaging, US, CT, and pathologic analysis. Radiographics 2004; 24:3-17; discussion 18-19.

45. Terkivatan T, van den Bos IC, Hussain SM, Wielopolski PA, de Man RA, JN IJ. Focal nodular hyperplasia: lesion characteristics on state-ofthe-art MRI including dynamic gadolinium-enhanced and superparamagnetic iron-oxide-uptake sequences in a prospective study. $J$ Magn Reson Imaging 2006; 24:864-872.

46. Wang J, Chen LT, Tsang YM, Liu TW, Shih TT. Dynamic contrast-enhanced MRI analysis of perfusion changes in advanced hepatocellular carcinoma treated with an antiangiogenic agent: a preliminary study. AJR Am J Roentgenol 2004; 183:713-719.

47. Folkman J. Tumor angiogenesis: therapeutic implications. N Engl J Med 1971; 285:1182-1186.

48. Leach MO, Brindle KM, Evelhoch JL, et al. Assessment of antiangiogenic and antivascular therapeutics using MRI: recommendations for appropriate methodology for clinical trials. Br J Radiol 2003; 76 Spec No 1:S87-91.

49. Morgan B, Thomas AL, Drevs J, et al. Dynamic contrast-enhanced magnetic resonance imaging as a biomarker for the pharmacological response of PTK787/ZK 222584, an inhibitor of the vascular endothelial growth factor receptor tyrosine kinases, in patients with advanced colorectal cancer and liver metastases: results from two phase I studies. J Clin Oncol 2003; 21:3955-3964. 
Creteraral Discussion 


\section{General Discussion}

\section{Role of MRI in the staging and treatment of prostate cancer}

rostate cancer affects men of all races, cultures and ethnic backgrounds and is a major public health burden as well as an economic burden throughout the world (1-3). It is the most common non-cutaneous cancer and the third leading cause of cancer death in men in the United State and European Union (1, 3, 4). An estimated 234,460 new cases of prostate cancer will be diagnosed in the United States and 27,350 people will die of the disease in 2006 (1). A wide discrepancy exists between the number of men diagnosed and those dying from prostate cancer. In East Asian countries such as Japan, prostate cancer mortality remains lower than in Western industrial ccountries, but it has been continuously and dramatically rising over the past 40 years (5). At routine autopsies prostate cancer can be detected in $8.2-10 \%$ of men $(6,7)$. Prostate cancer is an age-related disease and our population is aging rapidly, so the number of new cases will grow substantially $(1,8)$.

The high incidence of prostate cancer, combined with earlier detection, downstaging at the time of diagnosis, and the slow natural progression of the disease, has made its management a complex and controversial issue (1, 9-12). The American Cancer Society found that from 1995 to 2001, 91\% of prostate cancer cases were of a local or regional stage at diagnosis, as compared with 74\% from 1986 to 1991 , and patients had a 5-year relative (ie, adjusted for life expectancy) survival rate of 100\% from 1995 to 2001 (1) (13). The 100\% 5-year relative survival rate for all stages indicates that prostate tumors have a slow growth rate and allow for prolonged survival, even in patients with metastases at diagnosis $(14,15)$. However, the downstaging has been accompanied by an unfortunate trend of overdetection and overtreatment of biologically insignificant disease (16-19). As localized prostate cancer more frequently contains biologically indolent features and patient and physician anxiety and societal pressures favor active intervention, a large population of men is being 'overtreated' (16). Autopsy studies indicated that the "overdetected" cancers never impacted patient longevity (17). Primary therapies with curative intent (surgery or radiation) provide excellent long-term cancer control but are accompanied by a risk of treatment-related morbidity. Conversely, the understandable appeal of watchful waiting or active surveillance is balanced by the potential harm of missing a window of curative opportunity for a cancer destined to progress (20). One challenge clinicians and patients face in the modern PSA era is to differentiate men who have disease destined to progress and cause morbidity/ mortality from those who will not require immediate, or possibly even delayed, therapeutic intervention (21-23).

Depending on patient age at diagnosis, the stage and aggressiveness of the tumor, the potential side-effects of the treatment, and patient comorbidity (24-26), the options for treatment may include deferred therapy, androgen ablation, radical surgery, and various forms of radiation therapy (brachytherapy, external beam irradiation, including intensity-modulated radiation therapy (IMRT), and combinations) $(27,28)$. Recent studies have suggested that most patients with clinically localized prostate cancer will undergo some type of local therapy in an attempt to cure the disease (29-42). Radical prostatectomy is still the most commonly recommended treatment for patients with a life expectancy of at least 10 years $(9,43)$. Because the most common treatment side-effects - erectile dysfunction and urinary incontinence - are serious, there is a growing demand for patient-specific therapies that can reduce treatment morbidity while maximizing treatment benefits $(44,45)$. Patients with prostate cancer have to choose from a wide range of treatments, and the decision significantly affects both quality of life and survival. An important objective prior to any cancer therapy is to obtain a comprehensive and accurate knowledge of tumor location, size, locoregional extent, and biologic potential. Better tools are needed to help physicians and patients decide what type of treatment is most appropriate, or whether any treatment is needed at all (10, 46-48).

There are a number of clinical parameters and clinical nomograms to help with the choice of treatment $(45,49-54)$. To aid in patient counseling, the National Comprehensive Cancer Network (NCCN) guidelines define low risk as a PSA less than $10 \mathrm{ng} / \mathrm{mL}$, a Gleason score of 6 or lower, and a T stage of T2a or lower; the guidelines define high risk as a PSA of more than $20 \mathrm{ng} / \mathrm{mL}$, a Gleason score of 8 or higher, or T2c; intermediate risk is defined as a PSA of 10 to 20 $\mathrm{ng} / \mathrm{mL}$, T2b, or a Gleason of 7 (55). Patients with low-risk disease may be treated with prostatectomy or radiotherapy alone. Patients with intermediate risk disease are usually treated with radiotherapy and a short duration (less than 6 months) of hormonal ablation (medical castration using a gonadotropin-releasing hormone analog), and those with high-risk disease are usually treated with radiotherapy and a long duration of hormonal ablation $(37,56)$. To replace somewhat arbitrary combinations of individual clinical variables, nomograms have been developed that give a prediction of the final pathologic stage and an estimate of the chances of freedom from disease recurrence and aid in the choice of treatment $(45,52-54,57-60)$. The Partin staging nomogram (also called the "Partin Tables"), 
which is based on clinical stage, Gleason score, and serum PSA level, was first published in 1993 and was updated in 1997 and again in 2001 to predict the pathological stage at radical prostatectomy in men with prostate cancer (57, 59, 60). Other nomograms, such as Kattan's nomograms, have been developed to predict stage, individual features of stage (e.g., SVI, ECE), or recurrence, using variables such as the extent and location of cancer in systemic biopsy results, the percentage or number of biopsy cores positive for cancer, or the highest Gleason sum in any core (43, 52,61-63). As an important advance in accurate prediction for clinical medicine, the nomograms allow calculation of the continuous probability of a particular outcome and tend to outperform both expert clinicians and predictive instruments based on risk grouping $(45,59)$. The nomograms are validated predictive instruments widely used for individual patient counseling and important decision making (64-70). Despite the strong predictive ability and the cost-effectiveness of the nomograms, there is room for improved accuracy of prediction, particularly since clinical staging in the nomograms, is based only on digital rectal examination (DRE) and biopsy-determined Gleason grade, although valuable, is subject to sampling error (71-74). Moreover, the nomograms are limited because they do not incorporate the results of imaging studies that could guide interventions to control local disease. Thus, a technique that noninvasively demonstrates the presence, extent, and aggressiveness of prostate cancer could contribute incremental value to clinical nomograms and variables and make a substantial contribution to the decision-making process for individualized treatment $(46-48,75,76)$.

Although reports on the value of MRI in prostate cancer management have been contradictory, there is no doubt that MRI has an essential role to play in making safer, more individualized therapies possible (10, 77-80). In the last decade MRI has improved considerably with technologic refinements and increased reader experience. State-ofthe-art techniques, such as use of an endorectal coil, MR spectroscopic imaging (MRSI), faster imaging sequences, more powerful gradient coils, image post-processing and picture and communication systems (PACS) provide greater resources for improved interpretation of MR images of the prostate by experienced radiologists (46-48, 75, 81-86). The determination of treatment options should benefit from an imaging modality that allows non-invasive visualization of the primary tumor and measurement of its extent. This dissertation addresses the ability of state-of-the-art MRI/MRSI to non-invasively improve prostate cancer staging, treatment planning, and evaluation of recurrence-free probabilities and aggressiveness, together with the potential clinical application of color-coded parameter imaging derived from postprocessing of dynamic multiphasic gadolinium-enhanced MRI (DMGE-MRI) of abdominal tumors. First the role of MRI and MRSI in prostate cancer staging will be discussed, with specific attention to the detection of organ-confined prostate cancer (OCPC), extracapsular extension (ECE), seminal vesicle invasion $(\mathrm{SVI})$, and lymph node metastasis (LNM). Then the uses of MRI in pre-surgical planning will be examined, and the effect of MRI monitoring on recurrence rates and on the determination of cancer aggressiveness - as well as some post-processing issues - will be discussed. Finally, I shall make recommendations for diagnostic MRI of prostate cancer based on this overview of the literature.

\section{Role of MRI/MRSI in prostate cancer staging}

While historically, the staging of prostate cancer was based on the Jewett classification system, in the last 10 years TNM (tumor-node-metastasis) staging has prevailed. At present, however, TNM staging system is in common use particularly in the USA. TNM staging of prostate cancer has undergone a number of modifications, the latest ones having been made in 2002 by the American Joint Committee on Cancer (AJCC). The 2002 revised TNM system, shown in Table 1, is clinically useful and precisely stratifies newly diagnosed cancer (87). Most importantly, clinicians must distinguish between patients with pathologically organ-confined prostate cancer (OCPC) (pT2a-b) considered good surgical candidates and those with non-organ-confined prostate cancer (pT3-4). If cancer extends outside the prostate, the chances of cure are substantially diminished and the surgical or radiation treatment planning must be adapted to ensure complete eradication of the cancer (88).

TNM staging of prostate cancer is largely dependent on imaging. Due to high spatial resolution, superior contrast resolution, multiplanar capability, and a large field of view, MRI has higher accuracy in the assessment of unilateral or bilateral OCPC (pT2a-b), ECE (pT3a) and SVI (pT3b), and the invasion of adjacent structures (stage T4) than CT, ultrasound and DRE. MR spectroscopy indirectly increases the accuracy of MR staging by providing information on tumor volume and location and shows great promise in the differentiation of indolent from aggressive disease $(46-48,75,89-93)$. This MR information can help patients make the best decision about which treatment to select and can help the surgeon plan the operation to be sure all cancer is removed while the critical nerves and the sphincter are not removed. Other advantages of improved cancer staging include a better stratification of patients in clinical trials, the possibility of monitoring the progress of patients who select watchful waiting 
Table 1. TNM Staging of Prostate Cancer (AJCC 6th edition (2002)(87)

\begin{tabular}{|c|c|}
\hline \multicolumn{2}{|c|}{ Evaluation of the (primary) tumor (' $T$ ') } \\
\hline TX & cannot evaluate the primary tumor \\
\hline TO & no evidence of tumor \\
\hline T1 & tumor present, but not detectable clinically or with imaging \\
\hline T1a & tumor was incidentally found in less than $5 \%$ of prostate tissue resected (for other reasons) \\
\hline $\mathrm{T} 1 \mathrm{~b}$ & tumor was incidentally found in greater than $5 \%$ of prostate tissue resected \\
\hline T1c & tumor was found in a needle biopsy performed due to an elevated serum PSA \\
\hline T2 & the tumor can be felt (palpated) on examination, but has not spread outside the prostate \\
\hline T2a & the tumor is in half or less than half of one of the prostate gland's two lobes \\
\hline $\mathrm{T} 2 \mathrm{~b}$ & the tumor is in more than half of one lobe, but not both \\
\hline $\mathrm{T} 2 \mathrm{C}$ & the tumor is in both lobes \\
\hline T3 & the tumor has spread through the prostatic capsule (if it is only part-way through, it is still T2) \\
\hline Т3а & the tumor has spread through the capsule on one or both sides \\
\hline $\mathrm{T} 3 \mathrm{~b}$ & the tumor has invaded one or both seminal vesicles \\
\hline T4 & $\begin{array}{l}\text { the tumor has invaded adjacent structures other than seminal vesicles (e.g., bladder neck, } \\
\text { external sphincter, rectum, levator muscles, and/or pelvic wall) }\end{array}$ \\
\hline \multicolumn{2}{|c|}{ Evaluation of the regional lymph nodes ('N') } \\
\hline NX & cannot evaluate the regional lymph nodes \\
\hline NO & there has been no spread to the regional lymph nodes \\
\hline N1 & there has been spread to the regional lymph nodes \\
\hline \multicolumn{2}{|c|}{ Evaluation of distant metastasis ('M') } \\
\hline MX & cannot evaluate distant metastasis \\
\hline Mo & there is no distant metastasis \\
\hline M1 & there is distant metastasis \\
\hline M1a & the cancer has spread to lymph nodes beyond the regional ones \\
\hline M1b & the cancer has spread to bone \\
\hline M1c & the cancer has spread to other sites (regardless of bony involvement) \\
\hline
\end{tabular}

or other minimally aggressive cancer management options, and the guidance and assessment of emerging local prostate cancer therapies. However, the literature shows a wide range (from 50\% to 92\%) in the accuracy of local staging by MRI. Despite the high specificity of MRI, its widely varying sensitivity and substantial interobserver variability (ECE: specificity 47-99\%, sensitivity 22-80\%; SVI: specificity $81-99 \%$, sensitivity 23-80\%; LNM: specificity 78.7$98.46 \%$, sensitivity $0-69 \%)(10,46-48,75,76,80,84,89,94-110)$ make its routine use in the local staging of prostate cancer controversial.

\section{(1) Detection of OCPC (pT2a-b)}

A cancer completely confined to the prostate is defined as pT2a-b. After radical prostatectomy (RP), patients with pT2a-b cancer have an excellent prognosis, as more than $90 \%$ of them are free from biochemical recurrence at 5 years (9). Pretreatment knowledge of OCPC is important for treatment selection and planning, regardless of whether the treatment method ultimately chosen is watchful waiting, surgery, or radiation therapy (111-115). Radical prostatectomy in patients with pathologically organ-confined cancer results in a survival rate comparable with that of age-matched controls without prostate cancer $(52,116)$. The study described in Chapter 2 investigated the contribution of MR to the Partin Tables in predicting OCPC in 229 patients who underwent endorectal MRI and 383 who 
underwent combined endorectal MRI-MRSI before radical prostatectomy (46). Since the introduction of the Partin Tables in 1997, investigators have repeatedly validated the nomograms' capacity to help predict the pathologic stage of clinically localized prostate cancer (64-66). In 2001, the nomograms were updated based on a more contemporary cohort of disease features (57). The accuracy of the staging nomograms in predicting OCPC is high, with reports of the area under the ROC curve ranging from 0.79 to 0.82 (64-66). Despite the strong predictive ability and the cost-effectiveness of the staging nomograms, there is room for improved accuracy of prediction, particularly since clinical staging in the staging nomograms is based only on digital rectal examination. Moreover, the staging nomograms cannot assist in the localization of ECE, which is critical for optimal treatment planning $(57,117)$.

The data demonstrated that MR findings contributed significant incremental value $(P \leq .02)$ to the nomograms in the overall study population. The contribution of MR findings was significant in all risk groups but was greatest in the intermediate- and high-risk groups ( $\mathrm{P}<.01$ for both). Overall, in the prediction of OCPC, the area under the ROC curve for the staging nomograms was 0.80 , while the area under the ROC curve for the staging nomograms plus MR findings was 0.88 ; the difference was significant $(P<.01)$. In the combined endorectal MRI-MRSI group, the areas under the ROC curves were 0.81 for the staging nomograms and 0.90 for the staging nomograms plus MR findings; the difference was significant $(P<$.01).The contribution of MR spectroscopic imaging findings to endorectal MR imaging in the prediction of OCPC was also assessed in the study. The magnitude and extent of metabolic abnormality on MR spectroscopic images is indicative of tumor aggressiveness, volume, and stage $(118,119)$. Accuracy in the prediction of OCPC with MR was higher when MR spectroscopic imaging was used, but the difference was not significant. The results showed that MR findings (from endorectal MR imaging or combined endorectal MR imaging-MR spectroscopic imaging) contribute significant incremental value to clinical staging nomograms in the prediction of OCPC. The incorporation of endorectal MR imaging into future staging nomograms for the prediction of OCPC may therefore be warranted.

\section{(2) Detection of ECE (pT3a)}

A cancer that extends through the prostatic capsule into the periprostatic adipose tissue is defined as pT3a tumor (9). ECE is an important predictor of tumor progression because it is associated with greater risk of a positive surgical margin, recurrence and a decreased chance of long-term cancer control $(9,120-122)$. Accurate determination of ECE before treatment may alter treatment selection and planning $(52,121)$. Awareness of the presence and likely location of ECE would allow surgeons to plan radical prostatectomy more carefully, with the aim of resecting the cancer completely and minimizing the risk of damaging surrounding tissues important to recovery of sexual function $(52,81,123)$. The value of MRI staging was demonstrated in a 5-year follow-up study of 1,025 men with prostate cancer who were staged radiologically before prostatectomy (124). Endorectal MRI stages T3a (ECE) or T3b (SVI) had a significantly worse prognosis than endorectal MRI stages T2a-b (OCPC). A decision analysis model suggested that preoperative MRI was cost-effective in patients with a moderate or high risk of ECE (95). A multivariate analysis of endorectal MRI findings and other preoperative variables (PSA level, clinical stage, Gleason score, percentage of cancer in biopsy cores, and perineural invasion) in Chapter 3 showed that endorectal MRI findings were significant presurgical predictors of ECE in patients with prostate cancer, and added incremental value to clinical variables (75). Areas under the ROC curves for two models, with and without endorectal MR imaging findings, were 0.838 and 0.772 , respectively ( $\mathrm{P}=$.022). Endorectal MRI findings had a larger AUC than any of the clinical or histologic variables, a high negative predictive value and a high positive predictive value $(0.743,83.8 \%$ and $74.5 \%$, respectively). On endorectal $\mathrm{MRI}$, the criteria for extracapsular extension include a contour deformity with a step-off or angulated margin; an irregular bulge or edge retraction; a breach of the capsule with evidence of direct tumor extension; obliteration of the recto-prostatic angle; and asymmetry of the neurovascular bundles $(78,105,108)$.

While transaxial planes of section are essential in the evaluation of ECE, the utility of combining transaxial and coronal plane images using PACS cross-referencing to facilitate the diagnosis of extracapsular extension was shown in the study described in Chapter 6 (84). The study investigated 255 consecutive patients who underwent endorectal MRI before radical prostatectomy. In detecting ECE, the two radiologists had higher AUCs using cross-referencing (their AUCs increased from 0.66 to 0.87 , and from 0.69 to $0.86 ; \mathrm{p}<0.001$ for both). The weighted kappa was 0.56 with MRI alone and 0.76 with cross-referencing, indicating fair to good inter-reader agreement. Sensitivity/specificity for ECE with MRI alone and with cross-referencing, respectively, was 44\%/85\% and 68\%/95\% for reader 1 and 56\%/78\% and 74\%/95 for reader 2 . 
As medicine advances, ever-narrowing subspecialties evolve, and patients benefit from the added clinical expertise. Interpretation of prostate MRI examinations is important, and limited reader experience remains a barrier to greater acceptance of MRI use for preoperative imaging in many centers. Early reports demonstrated interobserver variability in MR imaging findings (125-127). The addition of MRSI to MRI has been shown to significantly increase staging accuracy for inexperienced readers and thus reduce interobserver variability (89). Chapter 4 reviewing preprostatectomy endorectal MRI findings for 344 consecutive patients with biopsy-proven prostate cancer showed that, overall, endorectal MRI findings were significant predictors of ECE and added significant incremental value to clinical variables when the images were interpreted by genitourinary radiologists experienced in MRI of the prostate (AUC 0.854 vs. $0.760, P=0.019$ ), but not when they were interpreted by general body MRI radiologists (AUC 0.813 vs.0.788, $P=0.31)(76)$. In the genitourinary MR imaging radiologists' group of patients, the AUC for endorectal MR imaging findings (0.833) was superior to that of all other predictors tested $(0.566-0.701)$. In the general body MR imaging radiologists' group of patients, AUC for endorectal MR imaging findings (0.646) was similar to that of the clinical predictors (0.582-0.793).This suggests that the recent improvement in the performance of MRI can be attributed to increased reader experience as well as to the maturation of MRI technology (e.g. faster imaging sequences, more powerful gradient coils, and post-processing image correction), and better understanding of morphological criteria used to diagnose ECE or SVI. These results may also explain the frustration over the use of endorectal MRI for prostate cancer evaluation.

\section{(3) Detection of SVI (pT3b)}

Seminal vesicle invasion (SVI) is defined as the extension of cancer into the muscular layer of the seminal vesicle (9). SVI is an important predictor of tumor progression because it is associated with increased risks of lymph node metastasis, recurrence (121, 122, 128-130). The criteria for SVI on endorectal MRI include contiguous low-signal-intensity tumor extension from the base of the gland into the seminal vesicles; tumor extension along the ejaculatory duct (non-visualization of the ejaculatory duct); asymmetric decrease in the signal intensity of the seminal vesicles; and decreased conspicuity of the seminal vesicle wall on T2 weighted images $(78,104)$. Prediction of SVI before treatment may influence treatment selection in favor of radiation therapy instead of surgery. Furthermore, while resection of the seminal vesicles has been a standard component of radical prostatectomy, it has recently been suggested that if SVI can be confidently ruled out, the surgeon may wish to spare the seminal vesicles during radical prostatectomy to prevent long-term loss of urinary continence (131).

Chapter 5 investigated 573 patients who underwent endorectal MRI before surgery for prostate cancer and had systematic needle biopsy results available for the base of the prostate (47). The results show that the Kattan nomogram (based on serum PSA, biopsy Gleason grade, clinical staging, and systematic needle biopsy cores from the base of the prostate) plus endorectal MRI (0.87) had a significantly larger AUC than either endorectal MRI alone (0.76) or the Kattan nomogram alone (0.80; $\mathrm{P}<0.05$ for both). On surgical histopathology $4.9 \%$ of the patients had evidence of $\mathrm{SVI}$. This result is consistent with the dramatic downstaging of prostate cancer at the time of diagnosis that has occurred over the last two decades (1). In the literature, serum PSA levels and Gleason grade are significantly associated with SVI on both univariate and multivariate analysis $(63,118,132)$. Our study results demonstrated that MRI, \%Ca (greatest percentage of cancer in all biopsy cores), PSA, clinical stage, Gleason grade, and PNI (perineural invasion) were all significantly associated with SVI on univariate analysis, while MRI, Gleason Grade, PSA and \%Ca were significantly associated with SVI on multivariate analysis. These results show that MRI can add significant incremental value to clinical variables in the prediction of SVI

Chapter 6 demonstrated the use of PACS cross-referencing, which automatically links axial, coronal and sagittal planes of section, to facilitate noninvasive evaluation of SVI (84). We investigated 255 consecutive patients who underwent endorectal MRI before radical prostatectomy. In detecting SVI, the AUCs of the two radiologists increased with cross-referencing (from 0.62 to $0.87, p=0.007$ and from 0.73 to $0.90, p=0.056$ for readers 1 and 2 , respectively). Sensitivity/specificity for SVI with MRI alone and with cross-referencing, respectively, was 38\%/81\% and 62\%/93\% for reader 1 and 62\%/84\% and 69\%/94\% for reader 2. PACS cross-referencing is particularly helpful in displaying the junction of the seminal vesicles and the central zone of the prostate. The results showed that PACS cross-referencing significantly improves the detection of prostate cancer SVI by 3D MRI. 


\section{(4) Detection of lymph node metastasis}

The presence of LNM at the time of prostate cancer diagnosis is associated with a high probability of progression after treatment and a poor prognosis (133-135). The risk of dying of prostate cancer at 10 years is much higher for patients with positive nodes than for patients with negative nodes (134). Pretreatment knowledge of prostate cancer LNM is important for patient counseling and appropriate treatment selection and planning. The PSA level recommended by the American Urological Association (AUA) for identifying patients who are at high risk for developing lymph node metastases is $15 \mathrm{ng} / \mathrm{mL}$. There is, however, a wide variation in the cut-off values for PSA reported in the literature (136). The conventional criterion for detection of metastatic lymph nodes on imaging is a short axis of $8 \mathrm{~mm}$ (137). MRI and CT have similar efficacy in detecting lymph node metastases, with both modalities having low sensitivity. The low sensitivity of MRI has been attributed mainly to the inability of cross-sectional imaging to detect metastases in normal-sized nodes $(137,138)$. Promising results by Harisinghani et al. have been reported for the use of ultra-small, super-paramagnetic iron oxide particles as an aid to diagnosing lymph node metastases (LNM) by MRI $(137,139)$. These particles are taken up by normal nodal tissue but not by metastatic tissue, providing tissue contrast within the lymph node and allowing detection of metastases. The sensitivity of MRI for LNM may be increased through use of these compounds, since they appear to permit detection of metastases in normal-sized nodes $(137,139)$. Chapter 7 described a study of 411 consecutive patients with clinically localized prostate cancer who underwent endorectal MRI before surgery (48). The overall positive lymph node rate of $5 \%$ confirmed the decreasing incidence of LNM in prostate cancer patients (1). The results showed that MRI was an independent statistically significant predictor of LNM ( $p=0.002)$, with sensitivity and specificity of $27.27 \%$ and $98.46 \%$, respectively, and positive predictive value and negative predictive value of 50\% and $95.99 \%$, respectively. On multivariate analysis, prediction of lymph node status using the model that included all MRI variables (ECE, SVI, and LNM) along with the Partin nomogram results had a significantly greater AUC than the univariate model that included only MRI LNM findings ( $A \cup C=0.892$ vs 0.633 , respectively; $p<0.01$ ). The study data confirmed the high negative predictive value of MRI for LNM and indicated that a combination of endorectal and phased-array MRI with the Partin tables had high accuracy in predicting LNM. As MRI also provides anatomic information that is helpful in treatment planning, these findings suggest that MRI, in conjunction with the Partin tables, may be useful for determining whether further imaging with lymphotropic superparamagnetic nanoparticles is warranted.

\section{The use of MRI in presurgical planning}

Risk-adjusted, patient-specific therapy designed to maximize cancer control while minimizing the risks of complications is being mandated $(10,81)$. Such an approach requires accurate characterization of the cancer location and extent. Optimal treatment for prostate cancer is best selected based on clinical TNM stage, Gleason grade and PSA level. The decision to treat should also take into account patient age, disease stage, associated medical illnesses, and the patient's personal preferences.

Historically, in 1905 Hugh Hampton Young reported radical prostatectomy utilizing a transperineal approach (140). In 1982 Patrick Walsh first described nerve sparing radical prostatectomy (141). The procedure was a revolutionary discovery to remove the prostate and seminal vesicles completely while preserving erectile function in previously potent men. Despite its obvious attractions, the nerve sparing procedure proved to be controversial $(116,142)$. In 1997, Peter Scardino, and Rahul Nath performed the first nerve grafting during a radical prostatectomy in order to restore sexual function after radical prostatectomy, though this procedure is still considered experimental (143-145).

Most cancers treated today are not palpable, and apart from the information obtained by ultrasound and biopsy, the surgeon has limited information about the size, location and extent of the cancer. Impotence (erectile dysfunction) is one of the possible complications after RP. Karakiewicz et al surveyed 2415 men treated for localized prostate cancer by radical prostatectomy and found a $75 \%$ rate of erectile dysfunction (146). Impotence after RP is quantitatively related to the resection of the neurovascular bundles (NVBs) that closely run along two sides of the prostate and control the blood flow to the penis and erections (147). However, prostate carcinoma most commonly arises in the peripheral zone of the prostate, often posteriorly, just beneath the capsule. A positive surgical margin reportedly increases the probability of disease recurrence $(121,148)$. Consequently, surgeons often resect widely in areas of suspected ECE to avoid a positive surgical margin $(144,149,150)$. In spite of all precautions, positive surgical margins are still present in approximately $23-31 \%$ of RP specimens $(149,151)$. The surgeon's ultimate goal is to excise the cancer completely while preserving the nearby normal structures, thus avoiding positive surgical margins and minimizing the chances of recurrence and allowing recovery of sexual function. Because the recovery of erectile function and the avoidance of positive surgical margins are important but competing outcomes, the decision to preserve or re- 
sect a neurovascular bundle during radical prostatectomy should be based on the most accurate information concerning the location and extent of the tumor. As we have seen from Chapters 2, 3, 5, and 7, the inclusion of MRI/MRSI findings in clinical nomograms and variables improves the prediction of cancer extent (OCPC, ECE, SVI and LNM), thereby improving patient selection for local therapy. Furthermore, as described in Chapter 8, information from MRI can assist in pre-surgical planning. The study involved 135 patients with biopsy proved prostate cancer (81). All patients underwent an assessment from the urologist as to the need for neurovascular bundle resection. This decision was based on DRE, PSA, biopsy results, percentage of positive biopsy cores, the percentage of tumor involved in each core and the Partin nomogram. After this assessment the urologist reviewed the MRI in consultation with the radiologist, and the surgeon's assessment of the need for neurovascular bundle (NVB) resection was recorded. The areas under the ROC curves were 0.741 for pre-MRI and 0.832 for post-MRI surgical planning ( $<<0.01)$. MRI findings suggested altering the surgical plan in 39\% (106/270) of NVBs. When the surgeon judged that the NVB resection was definitely not necessary (165 NVBs), MRI confirmed that decision in 138 NVBs (84\%); the concordant decision was correct in $96 \%$ of the cases (133 of 138 NVBs). In 36 high-risk patients ( $25 \%$ probability of organ-confined prostate cancer), MRI findings changed the surgical plan for $28 \mathrm{NVBs}$ (78\%); the change was found to be appropriate in 26 cases (93\%).The study found that MRI significantly improved the surgeon's decision to preserve or resect the neurovascular bundle(s). Such decisions are especially important in high-risk patient groups (81). MRI can help to refine the surgical plan, to maximize the preservation of periprostatic tissues (important for recovery of urinary and sexual function), and to minimize the risk of positive surgical margins. Thus, preoperative MRI can provide additional, critical information that allows treatment to be adjusted to an optimal, patient-specific therapy.

\section{MRI in the assessment of recurrence-free probabillities}

An estimated one third of patients treated with radical prostatectomy later experience biochemical recurrence as defined by increases in prostate-specific antigen (PSA) levels $(21,56,152)$. The natural history of biochemical recurrence after radical prostatectomy can be long but variable. Early identification, before detectable PSA is measured, of men likely to ultimately experience disease progression would be useful in considering early adjuvant therapy (153-156). Accurate identification of the risk of disease recurrence would also be particularly useful in clinical trials to assure comparability of treatment and control groups or to identify appropriate candidates for investigational treatment $(157,158)$. To aid in patient counseling, pre-operative nomograms have been developed that give an estimate of the chances of freedom from disease recurrence after RP rather than placing the patient in a risk group $(61,157)$. The nomograms are graphic representations of a statistical model, with scales for calculating the prognostic weight of a value for each individual variable $(54,157)$. Nomograms tend to outperform both expert clinicians and predictive instruments based on risk grouping. In general, nomograms are validated using patients treated at academic centers $(45,68,159)$. Although current nomograms are limited in their application and accuracy, efforts are continuously under way to improve them and to develop novel instruments for other end points.

Chapter 9 described a study that investigated whether the addition of MRI findings could significantly improve the accuracy of a preoperative nomogram for predicting biochemical recurrence, using post-operative predictions of biochemical recurrence as the standard of reference. The study included 592 consecutive patients with biopsyproven prostate cancer who were referred for endorectal MRI prior to RP. The design of the MRI scoring system in the study was based on the predicted pathologic stage; a higher score was assigned to each increase in stage, as it has been shown that higher pathologic stages are associated with higher rates of biochemical recurrence $(121,154)$. The 10-year biochemical recurrence-free probabilities for patients with OCPC, ECE, SVI and LNM were previously reported to be $92 \%, 71 \%, 37 \%$ and $7 \%$, respectively (121). Each of these parameters was a significant predictor of recurrence on multivariate analysis, and the ability of MRI to successfully identify each of these parameters has been demonstrated in chapters 2-7. The addition of MRI scores to the pre-operative nomogram significantly improved the R-square value of the pre-operative nomogram in the total study cohort ( $0.58 \mathrm{vs} .0 .53 ; p<0.01)$ and in a subset of patients (PSA $\geq 10$, Gleason score $\geq 7$ on biopsy or clinical stage T2) at high risk for biochemical recurrence (0.61. vs. $0.58 ; p<0.01)$. The study suggests that the addition of MRI findings can significantly improve the accuracy of the Kattan pre-operative nomogram in predicting postoperatively determined recurrence-free probabilities. 


\section{Role of MRI in assessing prostate cancer aggressiveness}

One of the most challenging characteristics of prostate cancer is its variable biologic aggressiveness, $(43,160-162)$. Noninvasive assessment of tumor aggressiveness for prostate cancer management may provide important diagnostic information and therefore help stratify patients for appropriate treatment (10).

The Gleason scoring system assigns increasing pattern grades as the glands formed by the prostate epithelial cells become more poorly differentiated, the margins of the tumor become more poorly defined, and the degree of stromal invasion increases $(163,164)$. The system remains one of the most powerful prognostic predictors of prostate cancer nearly 40 years after its initial description. It is endorsed as the primary grading system for prostate cancer by the World Health Organization (WHO), the Armed Forces Institute of Pathology Fascicle on Prostate Cancer, the Association of Directors of Anatomic and Surgical Pathology, and the College of American Pathologists (165). The Gleason grading system was updated at the 2005 United States and Canadian Academy of Pathology Annual Meeting (166-168).

Chapter 10 investigated seventy-four patients who underwent endorectal MRI before radical prostatectomy with subsequent whole-mount step-section pathology between January 2001 and July 2004 and had no prior treatment; at least one lesion with bi-dimensional diameter product $\geq 20 \mathrm{~mm}^{2}$ of uniform Gleason grade 3 or 4 or with Gleason grade 5 components; and no high signal intensity indicating postbiopsy changes on T1-weighted images. The results showed that Gleason grade correlated significantly $(p<0.05)$ with tumor-to-muscle signal intensity ratio for peripheral zone and transition zone tumors on corrected (Tau-b $=-0.50$ and -0.35 , respectively) and uncorrected (Tau-b $=-0.51$ and -0.33 , respectively) images. Higher Gleason grades were associated with lower tumor-to-muscle signal intensity ratios, although there was some overlap between the signal intensity ratios for tumors of different grades, perhaps due to tissue heterogeneity. Non-tumor-to-muscle signal intensity ratios did not correlate with patients' Gleason grades. Tumor-to-muscle signal intensity ratios were lower in transition zone than in peripheral zone tumors for all Gleason grade 3 lesions, $(p<0.001)$. This study demonstrates that T2-weighted endorectal MRI has the potential to allow noninvasive assessment of prostate cancer biological aggressiveness, which could help stratify patients for appropriate management.

\section{Color-coded parameter imaging derived from post-processing of DMGE-MRI of abdominal tumors}

Post-processing of dynamic multiphasic gadolinium-enhanced MRI (DMGE-MRI) of the abdomen allows generation of color-coded parameter images and time-intensity curves. Chapter 11 illustrates the current status and potential applications of automated and color-coded post processing techniques for analysis of dynamic multiphasic gadolinium-enhanced MR imaging of abdominal tumors.

The color-coded images are qualitative pixel-wise displays of various parameters, which are derived from the quantitative data of the time-intensity curves at each pixel or compartment pharmacokinetic modeling (169-171). Analysis of the time-intensity curves and the derived parameters are very helpful in quantitative characterization of tumor enhancement patterns and tumor angiogenesis related to tumor growth, grade, and metastatic potential (83, 171187). In addition, the parameters may also improve the specificity of MRI findings and help to facilitate monitoring of anti-tumor therapy response $(176,177,188)$.

Prostate cancer is hypervascular cancer. Typically prostate cancer will demonstrate earlier signal enhancement (higher wash-in rate than benign prostate tissue) and will lose much of its contrast in later phases (higher wash-out rate than benign prostate tissue), especially in the relatively less enhancing peripheral zone but a large overlap of the wash-in/out rates between prostate cancer and benign prostate tissue in transitional zone $(175,189-197)$. The optimal parameter is relative enhancement parameter for discrimination of cancer from normal tissue in peripheral / transitional zone tissue. The second best parameter is maximum enhancement in the peripheral zone and wash-out in the transitional zone (196). Color-coded parameter images can assist in determining established / minimal ECE in peripheral zone / transitional zone and SVI (198). Prostate cancer has a Type III curve. The time-intensity curves as well as the color-coded parameter are useful for prostate cancer detection and localization and staging and have potential in monitoring the effects of therapy and detecting tumor microvessel density and permeability $(175,184$, 190-194, 198, 199). 
The mechanisms behind the differential contrast enhancement are thought to include differences in tumor perfusion and the levels of tumor capillary wall permeability and hydrostatic pressure. The growth of a tumor, and its associated angiogenesis, is often rapid and disordered, leading to: 1) a relatively high fraction of immature blood vessels; 2) abnormal basal membranes in the vessel wall with relative lack of pericytes or smooth muscle associations with endothelial cells which render the vessels more "leaky" or permeable (providing an access point for contrast agents); 3) tumor vessels are tortuous, vary in diameter, and tend toward excessive branching and shunt formation; 4) intermittent or unstable blood flow with acutely collapsing vessels; and 5) hydrostatic pressure differences between tumors and normal tissue, partly reduced or absent lymphatics which may influence contrast agent uptake. Vascular endothelial growth factor, VEGF, has a role to play in increasing the permeability of these vessel walls. Blood volume, blood flow, mean transit time and microvascular permeability-surface area product are all, in principle, quantifiable through analysis of dynamic gadolinium-enhanced MRI by using pharmaco-kinetic models (170, 171, 176-178, 184, 186, 190-194, 198, 200-207). Tumor regions with dense neovasculature may enhance more rapidly compared to other regions. The position of these regions is related to the distribution of viable blood supply within the tumor. In necrotic or cystic regions, vascular density can be extremely low, whereas in highly active regions, the vascular density can be high.

The color-coded parameter images can provide information concerning focal or diffuse disease processes. This reduces the risk of missing areas of pathology, and reduces the risk of creating regions-of-interest (ROI) containing more than one tissue type, and makes further $\mathrm{ROI}$ analysis more accurate. In addition, the color-coded parameter images combine both the enhancement pharmaco-kinetics of tissues and morphology, and visualize the enhancement changes over time.

The parameters derived from the time-intensity curves, which are the basis of the color-coded parameter images, have contributions from contrast dose as well as MR pulse sequence parameters. Therefore, it is difficult to compare the results of studies from different institutions. Application of pharmaco-kinetic modeling has the potential to standardize the results from separate groups. The patient motion might lead to faulty time-signal-intensity curves at each pixel, and hence to faulty functional images.

The color-coded parameter images are limited by the necessary trade-off between temporal resolution, spatial resolution, and slice coverage. A high temporal resolution is needed at the expense of spatial resolution and slice coverage. When the color-coded parameters are sufficiently accurate, it could be possible to lower temporal resolution and therefore increase spatial resolution (which is essential in locating small tumors) and slice coverage (208).

\section{Prospects and recommendations for further research}

The 1.5-Tesla (1.5T) MR scanner used in the studies described in Chapters 2-11 is becoming more widely available in the routine clinical setting to depict and stage prostate cancer. The introduction of an endorectal coil and fast spinecho sequences enabled the acquisition of T2-weighted images of the prostate and its surrounding structures with a high spatial resolution within clinically acceptable examination times. Despite the relatively high spatial resolution that routinely can be acquired, Clearly there is still substantial room for improvement, as reflected in the widely varying staging accuracy (50\% to 92\%) with low sensitivity and considerable inter-reader variability (ECE: specificity 47-99\%, sensitivity 22-80\%) reported in the literature $(10,46-48,75,76,80,84,89,94-110)$.

\section{Future improvements in MR imaging: $3 T$, new coils}

"Science and technology is an endless frontier," the eminent American physicist Vannevar Bush once said. MRI as an imaging modality for one-stop prostate imaging remains in evolution, even revolution, and advances are continuously being made in accuracy $(86,208,209)$.

As 3T MR scanners become more available, body imaging at high field strength and improved coil design is becoming the subject of intensive research $(208,210-212)$. Theoretically, increasing static magnetic field strength (B0) from $1.5 T$ to $3 T$ will result in a theoretical doubling of the signal-to-noise ratio (SNR). This in turn can be used to achieve greater spatial resolution and/or reduce scanning time. However, chemical shift and susceptibility artifacts also increase linearly with B0. Improvements in coil design include the use of rigid coils with higher signal and the introduction of a susceptibility-matched agent into the rectum to reduce distortions relating to the interface of rectal air and tissue. The following advantages can be expected from imaging of prostate cancer with higher field strength and improved coil design: 
(1) Increased spatial resolution due to increased signal-to-noise ratio (SNR) may improve the detection of microscopic extracapsular extension. Microscopic extracapsular extension is (ECE) defined as the presence of no more than a few malignant cells immediately outside the capsule on no more than 2 sections. The 10-year risk of recurrence (clinical, radiological or biochemical) was 32\% in patients with microscopic ECE compared to $42 \%$ in patients with more established ECE, suggesting that microscopic ECE is nearly as important as macroscopic ECE with respect to prognosis (9).

(2) Higher field strength should provide increased spectral and spatial resolution for spectroscopic imaging, but new pulse sequences will have to be designed for overcoming field inhomogeneities and citrate J-modulation issues. Significant improvement in metabolite resolution in magnetic resonance spectroscopy with prostate cancer should be expected.

(3) Faster scanning may help to reduce image artifacts related to patient motion and rectal peristalsis.

(4) Finally, dynamic multiphasic gadolinium-enhanced MRI (DMGE-MRI) should achieve increased SNR and faster image acquisition, with a significantly better trade-off between temporal and spatial resolution.

\section{Recommendations for further research}

(1) Chapter 4 demonstrates that prostate cancer patients benefit from the subspecialization of radiologists in genitourinary imaging. Further study of the role of reader experience and the learning curve in the interpretation of prostate imaging would be helpful.

(2) The studies described in Chapters 2-5 and 7-9 were not designed to assess MRI technology, but to assess the value of MRI as applied in the clinical setting, during radiologists' regular clinical assignments. Accordingly, the readers had access to clinical data such as PSA level and biopsy results when available in the patient's medical record. As each exam was evaluated only once, interobserver and intraobserver variability could not be assessed. Further prospective multi-institutional studies would be helpful to confirm our findings. Such studies, however, should be designed with the knowledge that the quality of the images and the experience and training of the interpreter play an essential role in determining whether MRI can provide value in the care of patients with prostate cancer.

(3) Further research is needed to assess the potential clinical applications of 3T MRI scanners, new coils, and new MRI techniques (e.g., diffusion-weighted imaging, dynamic contrast-enhanced imaging, and multimodality MR imaging) in prostate cancer management.

\section{References}

1. Jemal A, Siegel R, Ward E, et al. Cancer statistics, 2006. CA Cancer J Clin 2006; 56:106-130.

2. Parkin DM, Bray F, Ferlay J, Pisani P. Global cancer statistics, 2002. CA Cancer J Clin 2005; 55:74-108.

3. Kamangar F, Dores GM, Anderson WF. Patterns of cancer incidence, mortality, and prevalence across five continents: defining priorities to reduce cancer disparities in different geographic regions of the world. J Clin Oncol 2006; 24:2137-2150.

4. Boyle P, Ferlay J. Cancer incidence and mortality in Europe, 2004. Ann Oncol 2005; 16:481-488.

5. Marugame T, Mizuno S. Comparison of prostate cancer mortality in five countries: France, Italy, Japan, UK and USA from the WHO mortality database (1960-2000). Jpn J Clin Oncol 2005; 35:690-691.

6. Bubendorf L, Schopfer A, Wagner U, et al. Metastatic patterns of prostate cancer: an autopsy study of 1,589 patients. Hum Pathol 2000 ; 31:578-583.

7. Gatling RR. Prostate carcinoma: an autopsy evaluation of the influence of age, tumor grade, and therapy on tumor biology. South Med $J$ 1990; 83:782-784.

8. Scardino PT. New technology and the changing world of cancer. Nat Clin Pract Urol 2005; 2:403.

9. Ohori M, Kattan M, Scardino PT, Wheeler TM. Radical prostatectomy for carcinoma of the prostate. Mod Pathol 2004; 17:349-359.

10. Hricak H. MR imaging and MR spectroscopic imaging in the pre-treatment evaluation of prostate cancer. Br J Radiol 2005; 78 Spec No 2: S103-111.

11. Galper SL, Chen MH, Catalona WJ, Roehl KA, Richie JP, D'Amico AV. Evidence to support a continued stage migration and decrease in prostate cancer specific mortality. J Urol 2006; 175:907-912.

12. Stamey TA, Caldwell M, McNeal JE, Nolley R, Hemenez M, Downs J. The prostate specific antigen era in the United States is over for prostate cancer: what happened in the last 20 years? J Urol 2004; 172:1297-1301.

13. Parker SL, Tong T, Bolden S, Wingo PA. Cancer statistics, 1996. CA Cancer J Clin 1996; 46:5-27.

14. Varenhorst E, Garmo H, Holmberg L, et al. The National Prostate Cancer Register in Sweden 1998-2002: trends in incidence, treatment and survival. Scand J Urol Nephrol 2005; 39:117-123.

15. Han M, Partin AW, Pound CR, Epstein J, Walsh PC. Long-term biochemical disease-free and cancer-specific survival following anatomic radical retropubic prostatectomy. The 15-year Johns Hopkins experience. Urol Clin North Am 2001; 28:555-565. 
16. Carroll PR. Early stage prostate cancer--do we have a problem with over-detection, overtreatment or both? J Urol 2005; 173:1061-1062.

17. Konety BR, Bird VY, Deorah S, Dahmoush L. Comparison of the incidence of latent prostate cancer detected at autopsy before and after the prostate specific antigen era. J Urol 2005; 174:1785-1788; discussion 1788.

18. Cooperberg MR, Lubeck DP, Mehta SS, Carroll PR. Time trends in clinical risk stratification for prostate cancer: implications for outcomes (data from CaPSURE). J Urol 2003; 170:S21-25; discussion S26-27.

19. Freedland SJ, Kane CJ, Amling CL, Aronson WJ, Presti JC, Jr., Terris MK. Delay of radical prostatectomy and risk of biochemical progression in men with low risk prostate cancer. J Urol 2006; 175:1298-1302; discussion 1302-1293.

20. Harlan SR, Cooperberg MR, Elkin EP, et al. Time trends and characteristics of men choosing watchful waiting for initial treatment of localized prostate cancer: results from CaPSURE. J Urol 2003; 170:1804-1807.

21. Lieberman R. Evidence-based medical perspectives: the evolving role of PSA for early detection, monitoring of treatment response, and as a surrogate end point of efficacy for interventions in men with different clinical risk states for the prevention and progression of prostate cancer. Am J Ther 2004; 11:501-506.

22. Freedland SJ, Partin AW. Prostate-specific antigen: update 2006. Urology 2006; 67:458-460.

23. Schroder FH. Prostate specific antigen and other markers for prostate cancer. J Urol 2006; 175:1199-1200.

24. Bill-Axelson A, Holmberg L, Ruutu M, et al. Radical prostatectomy versus watchful waiting in early prostate cancer. N Engl J Med 2005; 352:1977-1984.

25. Lu-Yao GL, Yao SL. Population-based study of long-term survival in patients with clinically localised prostate cancer. Lancet 1997; 349:906910.

26. Leibowitz RL, Tucker SJ. Treatment of localized prostate cancer with intermittent triple androgen blockade: preliminary results in 110 consecutive patients. Oncologist 2001; 6:177-182.

27. Gilligan T, Kantoff PW. Chemotherapy for prostate cancer. Urology 2002; 60:94-100; discussion 100.

28. Thompson IM, Seay TM. Will current clinical trials answer the most important questions about prostate adenocarcinoma? Oncology (Williston Park) 1997; 11:1109-1117; discussion 1117-1108, 1121

29. Zelefsky MJ, Moughan J, Owen J, Zietman AL, Roach M, 3rd, Hanks GE. Changing trends in national practice for external beam radiotherapy for clinically localized prostate cancer: 1999 Patterns of Care survey for prostate cancer. Int J Radiat Oncol Biol Phys 2004; 59:1053-1061.

30. Rukstalis DB, Goldknopf JL, Crowley EM, Garcia FU. Prostate cryoablation: a scientific rationale for future modifications. Urology 2002; 60:19-25.

31. Kennedy JE. High-intensity focused ultrasound in the treatment of solid tumours. Nat Rev Cancer 2005; 5:321-327.

32. Blana A, Walter B, Rogenhofer S, Wieland WF. High-intensity focused ultrasound for the treatment of localized prostate cancer: 5-year experience. Urology 2004; 63:297-300

33. Uchida T, Ohkusa H, Nagata Y, Hyodo T, Satoh T, Irie A. Treatment of localized prostate cancer using high-intensity focused ultrasound BJU Int 2006; 97:56-61.

34. Leibovich BC, Blute ML, Bostwick DG, et al. Proximity of prostate cancer to the urethra: implications for minimally invasive ablative therapies. Urology 2000; 56:726-729.

35. Han KR, Cohen JK, Miller RJ, et al. Treatment of organ confined prostate cancer with third generation cryosurgery: preliminary multicenter experience. J Urol 2003; 170:1126-1130.

36. Bahn DK, Lee F, Badalament R, Kumar A, Greski J, Chernick M. Targeted cryoablation of the prostate: 7-year outcomes in the primary treatment of prostate cancer. Urology 2002; 60:3-11.

37. D'Amico AV, Whittington R, Malkowicz SB, et al. Biochemical outcome after radical prostatectomy or external beam radiation therapy for patients with clinically localized prostate carcinoma in the prostate specific antigen era. Cancer 2002; 95:281-286.

38. Onik G, Narayan P, Vaughan D, Dineen M, Brunelle R. Focal "nerve-sparing" cryosurgery for treatment of primary prostate cancer: a new approach to preserving potency. Urology 2002; 60:109-114.

39. Oleinick NL, Evans HH. The photobiology of photodynamic therapy: cellular targets and mechanisms. Radiat Res 1998; $150:$ S146-156.

40. Kelleher DK, Thews O, Scherz A, Salomon Y, Vaupel P. Perfusion, oxygenation status and growth of experimental tumors upon photodynamic therapy with Pd-bacteriopheophorbide. Int J Oncol 2004; 24:1505-1511.

41. Koudinova NV, Pinthus JH, Brandis A, et al. Photodynamic therapy with Pd-Bacteriopheophorbide (TOOKAD): successful in vivo treatment of human prostatic small cell carcinoma xenografts. Int J Cancer 2003; 104:782-789.

42. Nathan TR, Whitelaw DE, Chang SC, et al. Photodynamic therapy for prostate cancer recurrence after radiotherapy: a phase I study. J Urol 2002; 168:1427-1432.

43. Kattan MW, Wheeler TM, Scardino PT. Postoperative nomogram for disease recurrence after radical prostatectomy for prostate cancer. J Clin Oncol 1999; 17:1499-1507.

44. Bianco FJ, Jr., Scardino PT, Eastham JA. Radical prostatectomy: long-term cancer control and recovery of sexual and urinary function ("trifecta"). Urology 2005; 66:83-94.

45. Kattan MW, Scardino PT. Prediction of progression: nomograms of clinical utility. Clin Prostate Cancer 2002; 1:90-96.

46. Wang L, Hricak H, Kattan MW, Chen HN, Scardino PT, Kuroiwa K. Prediction of organ-confined prostate cancer: incremental value of MR imaging and MR spectroscopic imaging to staging nomograms. Radiology 2006; 238:597-603.

47. Wang L, Hricak H, Kattan MW, et al. Incremental value of endorectal MRI to the Kattan nomogram in the prediction of seminal vesicle invasion of prostate cancer. Radiology 2006; in press.

48. Wang L, Hricak H, Kattan MW, et al. Combined endorectal and phased-array MRI in the prediction of pelvic lymph node metastasis in prostate cancer. AJR Am J Roentgenol 2006; 186:743-748.

49. Perrotti M. Understanding PSA and prostate cancer risk assessment. N J Med 2001; 98:35-38.

50. Graefen M, Ohori M, Karakiewicz Pl, et al. Assessment of the enhancement in predictive accuracy provided by systematic biopsy in predicting outcome for clinically localized prostate cancer. J Urol 2004; 171:200-203.

51. Parsons JK, Partin AW. Clinical interpretation of prostate biopsy reports. Urology 2006; 67:452-457.

52. Ohori M, Kattan MW, Koh H, et al. Predicting the presence and side of extracapsular extension: a nomogram for staging prostate cancer. J Urol 2004; 171:1844-1849; discussion 1849.

53. Di Blasio CJ, Rhee AC, Cho D, Scardino PT, Kattan MW. Predicting clinical end points: treatment nomograms in prostate cancer. Semin Oncol 2003; 30:567-586.

54. Diblasio CJ, Kattan MW. Use of nomograms to predict the risk of disease recurrence after definitive local therapy for prostate cancer. Urology 2003; 62 Suppl 1:9-18.

55. Scherr D, Swindle PW, Scardino PT. National Comprehensive Cancer Network guidelines for the management of prostate cancer. Urology 2003; 61:14-24. 
56. Kattan MW, Zelefsky MJ, Kupelian PA, Scardino PT, Fuks Z, Leibel SA. Pretreatment nomogram for predicting the outcome of threedimensional conformal radiotherapy in prostate cancer. J Clin Oncol 2000; 18:3352-3359.

57. Partin AW, Mangold LA, Lamm DM, Walsh PC, Epstein Jl, Pearson JD. Contemporary update of prostate cancer staging nomograms (Partin Tables) for the new millennium. Urology 2001; 58:843-848.

58. Kattan MW, Wheeler TM, Scardino PT. Postoperative nomogram for disease recurrence after radical prostatectomy for prostate cancer. $J$ Clin Oncol 1999; 17:1499-1507

59. Partin AW, Kattan MW, Subong EN, et al. Combination of prostate-specific antigen, clinical stage, and Gleason score to predict pathological stage of localized prostate cancer. A multi-institutional update. Jama 1997; 277:1445-1451.

60. Khan MA, Partin AW. Partin tables: past and present. BJU Int 2003; 92:7-11.

61. Kattan MW, Eastham JA, Stapleton AM, Wheeler TM, Scardino PT. A preoperative nomogram for disease recurrence following radical prostatectomy for prostate cancer. J Natl Cancer Inst 1998; 90:766-771.

62. Gancarczyk KJ, Wu H, McLeod DG, et al. Using the percentage of biopsy cores positive for cancer, pretreatment PSA, and highest biopsy Gleason sum to predict pathologic stage after radical prostatectomy: the Center for Prostate Disease Research nomograms. Urology 2003; 61:589-595

63. Koh H, Kattan MW, Scardino PT, et al. A nomogram to predict seminal vesicle invasion by the extent and location of cancer in systematic biopsy results. J Urol 2003; 170:1203-1208

64. Blute ML, Bergstralh EJ, Partin AW, et al. Validation of Partin tables for predicting pathological stage of clinically localized prostate cancer. J Urol 2000; 164:1591-1595.

65. Augustin H, Eggert T, Wenske S, et al. Comparison of accuracy between the Partin tables of 1997 and 2001 to predict final pathological stage in clinically localized prostate cancer. J Urol 2004; 171:177-181.

66. Graefen M, Augustin H, Karakiewicz PI, et al. Can predictive models for prostate cancer patients derived in the United States of America be utilized in European patients? A validation study of the Partin tables. Eur Urol 2003; 43:6-10; discussion 11.

67. Graefen M, Karakiewicz PI, Cagiannos I, et al. Validation study of the accuracy of a postoperative nomogram for recurrence after radical prostatectomy for localized prostate cancer. J Clin Oncol 2002; 20:951-956

68. Graefen M, Karakiewicz PI, Cagiannos I, et al. International validation of a preoperative nomogram for prostate cancer recurrence after radical prostatectomy. J Clin Oncol 2002; 20:3206-3212

69. Greene $\mathrm{KL}$, Meng MV, Elkin EP, et al. Validation of the Kattan preoperative nomogram for prostate cancer recurrence using a community based cohort: results from cancer of the prostate strategic urological research endeavor (capsure). J Urol 2004; 171:2255-2259.

70. Penson DF, Grossfeld GD, Li YP, Henning JM, Lubeck DP, Carroll PR. How well does the Partin nomogram predict pathological stage after radical prostatectomy in a community based population? Results of the cancer of the prostate strategic urological research endeavor. $J$ Urol 2002; 167:1653-1657; discussion 1657-1658.

71. Nagler HM, Gerber EW, Homel P, et al. Digital rectal examination is barrier to population-based prostate cancer screening. Urology 2005; $65: 1137-1140$

72. Philip J, Dutta Roy S, Viswanathan P. Digital rectal examination is a barrier to population-based prostate cancer screening. Urology 2006; 67:655; author reply 655-656

73. Macias DJ, Sarabia MJ, Sklar DP. Male discomfort during the digital rectal examination: does examiner gender make a difference? Am J Emerg Med 2000; 18:676-678

74. Cookson MS, Fleshner NE, Soloway SM, Fair WR. Correlation between Gleason score of needle biopsy and radical prostatectomy specimen: accuracy and clinical implications. J Urol 1997; 157:559-562.

75. Wang L, Mullerad M, Chen HN, et al. Prostate cancer: incremental value of endorectal MR imaging findings for prediction of extracapsular extension. Radiology 2004; 232:133-139.

76. Mullerad M, Hricak H, Wang L, Chen HN, Kattan MW, Scardino PT. Prostate cancer: detection of extracapsular extension by genitourinary and general body radiologists at MR imaging. Radiology 2004; 232:140-146.

77. Huzjan R, Sala E, Hricak H. Magnetic resonance imaging and magnetic resonance spectroscopic imaging of prostate cancer. Nat Clin Pract Urol 2005; 2:434-442.

78. Claus FG, Hricak H, Hattery RR. Pretreatment evaluation of prostate cancer: role of MR imaging and $1 \mathrm{H}$ MR spectroscopy. Radiographics 2004; 24 Suppl 1:S167-180.

79. Trabulsi EJ, Merriam WG, Gomella LG. New imaging techniques in prostate cancer. Curr Urol Rep 2006; 7:175-180.

80. Engelbrecht MR, Jager GJ, Laheij RJ, Verbeek AL, van Lier HJ, Barentsz JO. Local staging of prostate cancer using magnetic resonance imaging: a meta-analysis. Eur Radiol 2002; 12:2294-2302.

81. Hricak $\mathrm{H}$, Wang L, Wei DC, et al. The role of preoperative endorectal magnetic resonance imaging in the decision regarding whether to preserve or resect neurovascular bundles during radical retropubic prostatectomy. Cancer 2004; 100:2655-2663.

82. Hricak H. Given the improvement in pelvic coils for MR, is an endorectal coil necessary to evaluate prostate carcinoma? AJR Am $J$ Roentgenol 1995; 165:733-734.

83. Huch Boni RA, Boner JA, Lutolf UM, Trinkler F, Pestalozzi DM, Krestin GP. Contrast-enhanced endorectal coil MRI in local staging of prostate carcinoma. J Comput Assist Tomogr 1995; 19:232-237.

84. Wang L, Zhang J, Schwartz LH, et al. Incremental Value of Multiplanar Cross-Referencing for Prostate Cancer Staging by Endorectal MRI. AJR Am J Roentgenol 2006; in press.

85. Coakley FV, Kurhanewicz J, Lu Y, et al. Prostate cancer tumor volume: measurement with endorectal MR and MR spectroscopic imaging. Radiology 2002; 223:91-97.

86. Coakley FV, Qayyum A, Kurhanewicz J. Magnetic resonance imaging and spectroscopic imaging of prostate cancer. J Urol 2003; 170:S6975: discussion $575-66$

87. Green FL, Page DL, Fleming ID, et al. (eds.). AJCC Cancer Staging Manual. New York: Springer-Verlag, 2002.

88. Presti JC, Jr. Prostate cancer: assessment of risk using digital rectal examination, tumor grade, prostate-specific antigen, and systematic biopsy. Radiol Clin North Am 2000; 38:49-58

89. Yu KK, Scheidler J, Hricak H, et al. Prostate cancer: prediction of extracapsular extension with endorectal MR imaging and threedimensional proton MR spectroscopic imaging. Radiology 1999; 213:481-488

90. Zakian KL, Sircar K, Hricak H, et al. Correlation of proton MR spectroscopic imaging with gleason score based on step-section pathologic analysis after radical prostatectomy. Radiology 2005; 234:804-814.

91. Zakian $\mathrm{KL}$, Eberhardt S, Hricak H, et al. Transition zone prostate cancer: metabolic characteristics at $1 \mathrm{H}$ MR spectroscopic imaging--initial results. Radiology 2003; 229:241-247. 
92. Rajesh A, Coakley FV. MR imaging and MR spectroscopic imaging of prostate cancer. Magn Reson Imaging Clin N Am 2004; 12:557-579, vii.

93. Nakashima J, Tanimoto A, Imai Y, et al. Endorectal MRI for prediction of tumor site, tumor size, and local extension of prostate cancer. Urology 2004; 64:101-105.

94. Cornud F, Flam T, Chauveinc L, et al. Extraprostatic spread of clinically localized prostate cancer: factors predictive of pT3 tumor and of positive endorectal MR imaging examination results. Radiology 2002; 224:203-210.

95. Jager GJ, Severens JL, Thornbury JR, de La Rosette JJ, Ruijs SH, Barentsz JO. Prostate cancer staging: should MR imaging be used?--A decision analytic approach. Radiology 2000; 215:445-451.

96. Wallner K. MR imaging for prostate cancer staging: beauty or beast? Int J Radiat Oncol Biol Phys 2002; 52:886-887.

97. Sonnad SS, Langlotz CP, Schwartz JS. Accuracy of MR imaging for staging prostate cancer: a meta-analysis to examine the effect of technologic change. Acad Radiol 2001; 8:149-157.

98. May F, Treumann T, Dettmar P, Hartung R, Breul J. Limited value of endorectal magnetic resonance imaging and transrectal ultrasonography in the staging of clinically localized prostate cancer. BJU Int 2001; 87:66-69.

99. Ikonen $\mathrm{S}$, Karkkainen $\mathrm{P}$, Kivisaari $\mathrm{L}$, et al. Endorectal magnetic resonance imaging of prostatic cancer: comparison between fat-suppressed T2-weighted fast spin echo and three-dimensional dual-echo, steady-state sequences. Eur Radiol 2001; 11:236-241.

100. Rorvik J, Halvorsen OJ, Albrektsen G, Ersland L, Daehlin L, Haukaas S. MRI with an endorectal coil for staging of clinically localised prostate cancer prior to radical prostatectomy. Eur Radiol 1999; 9:29-34.

101. Perrotti M, Kaufman RP, Jr., Jennings TA, et al. Endo-rectal coil magnetic resonance imaging in clinically localized prostate cancer: is it accurate? J Urol 1996; 156:106-109.

102. Presti JC, Jr., Hricak H, Narayan PA, Shinohara K, White S, Carroll PR. Local staging of prostatic carcinoma: comparison of transrectal sonography and endorectal MR imaging. AJR Am J Roentgenol 1996; 166:103-108.

103. Bartolozzi C, Menchi I, Lencioni R, et al. Local staging of prostate carcinoma with endorectal coil MRI: correlation with whole-mount radical prostatectomy specimens. Eur Radiol 1996; 6:339-345.

104. Sala E, Akin O, Moskowitz CS, et al. Endorectal MR imaging in the evaluation of seminal vesicle invasion: diagnostic accuracy and multivariate feature analysis. Radiology 2006; 238:929-937.

105. Outwater EK, Petersen RO, Siegelman ES, Gomella LG, Chernesky CE, Mitchell DG. Prostate carcinoma: assessment of diagnostic criteria for capsular penetration on endorectal coil MR images. Radiology 1994; 193:333-339.

106. Harris RD, Schned AR, Heaney JA. Staging of prostate cancer with endorectal MR imaging: lessons from a learning curve. Radiographics 1995; 15:813-829; discussion 829-832.

107. Jager GJ, Ruijter ET, van de Kaa CA, et al. Local staging of prostate cancer with endorectal MR imaging: correlation with histopathology. AJR Am J Roentgenol 1996; 166:845-852.

108. Yu KK, Hricak H, Alagappan R, Chernoff DM, Bacchetti P, Zaloudek CJ. Detection of extracapsular extension of prostate carcinoma with endorectal and phased-array coil MR imaging: multivariate feature analysis. Radiology 1997; 202:697-702.

109. Chelsky MJ, Schnall MD, Seidmon EJ, Pollack HM. Use of endorectal surface coil magnetic resonance imaging for local staging of prostate cancer. J Urol 1993; 150:391-395.

110. Chefchaouni MC, Flam T, Cornud F, et al. [Results of endorectal MRI in local staging of prostatic cancer. Correlation with specimens from prostatectomy. Apropos of 47 cases]. J Urol (Paris) 1996; 102:51-55.

111. Graefen M, Haese A, Pichlmeier U, et al. A validated strategy for side specific prediction of organ confined prostate cancer: a tool to select for nerve sparing radical prostatectomy. J Urol 2001; 165:857-863.

112. Do V, Choo R, De Boer G, et al. The role of serial free/total prostate-specific antigen ratios in a watchful observation protocol for men with localized prostate cancer. BJU Int 2002; 89:703-709.

113. Hoff B, Pow-Sang JM. Observation in the management of localized prostate cancer. Cancer Control 2001; 8:151-154.

114. Brasso K, Friis S, Juel K, Jorgensen T, Iversen P. Mortality of patients with clinically localized prostate cancer treated with observation for 10 years or longer: a population based registry study. J Urol 1999; 161:524-528.

115. Moon TD, Brawer MK, Wilt TJ. Prostate Intervention Versus Observation Trial (PIVOT): a randomized trial comparing radical prostatectomy with palliative expectant management for treatment of clinically localized prostate cancer. PIVOT Planning Committee. J Natl Cancer Inst Monogr 1995:69-71.

116. Scardino PT. Continuing refinements in radical prostatectomy: more evidence that technique matters. J Urol 2005; 173:338-339.

117. Poulakis $V$, Witzsch $U$, de Vries $R$, et al. Preoperative neural network using combined magnetic resonance imaging variables, prostatespecific antigen, and gleason score for predicting prostate cancer biochemical recurrence after radical prostatectomy. Urology 2004; 64:1165-1170

118. Bostwick DG, Qian J, Schlesinger C. Contemporary pathology of prostate cancer. Urol Clin North Am 2003; 30:181-207.

119. Kurhanewicz J, Vigneron DB, Nelson SJ. Three-dimensional magnetic resonance spectroscopic imaging of brain and prostate cancer. Neoplasia 2000; 2:166-189.

120. Roach M, 3rd, Chen A, Song J, Diaz A, Presti J, Jr., Carroll P. Pretreatment prostate-specific antigen and Gleason score predict the risk of extracapsular extension and the risk of failure following radiotherapy in patients with clinically localized prostate cancer. Semin Urol Oncol 2000; 18:108-114.

121. Hull GW, Rabbani F, Abbas F, Wheeler TM, Kattan MW, Scardino PT. Cancer control with radical prostatectomy alone in 1,000 consecutive patients. J Urol 2002; 167:528-534.

122. Catalona WJ, Ramos CG, Carvalhal GF. Contemporary results of anatomic radical prostatectomy. CA Cancer J Clin 1999; 49:282-296.

123. Kamat AM, Jacobsohn KM, Troncoso P, Shen Y, Wen S, Babaian RJ. Validation of criteria used to predict extraprostatic cancer extension: a tool for use in selecting patients for nerve sparing radical prostatectomy. J Urol 2005; 174:1262-1265.

124. D'Amico AV, Whittington R, Malkowicz B, et al. Endorectal magnetic resonance imaging as a predictor of biochemical outcome after radical prostatectomy in men with clinically localized prostate cancer. J Urol 2000; 164:759-763.

125. Rifkin MD, Zerhouni EA, Gatsonis CA, et al. Comparison of magnetic resonance imaging and ultrasonography in staging early prostate cancer. Results of a multi-institutional cooperative trial. N Engl J Med 1990; 323:621-626.

126. Schiebler ML, Yankaskas BC, Tempany C, et al. MR imaging in adenocarcinoma of the prostate: interobserver variation and efficacy for determining stage $C$ disease. AJR Am J Roentgenol 1992; 158:559-562; discussion 563-554.

127. Tempany CM, Zhou X, Zerhouni EA, et al. Staging of prostate cancer: results of Radiology Diagnostic Oncology Group project comparison of three MR imaging techniques. Radiology 1994; 192:47-54. 
128. Masterson TA, Pettus JA, Middleton RG, Stephenson RA. Isolated seminal vesicle invasion imparts better outcomes after radical retropubic prostatectomy for clinically localized prostate cancer: Prognostic stratification of pt3b disease by nodal and margin status. Urology 2005.

129. Ramsden AR, Chodak G. An analysis of risk factors for biochemical progression in patients with seminal vesicle invasion: validation of Kattan's nomogram in a pathological subgroup. BJU Int 2004; 93:961-964.

130. Ohori M, Scardino PT, Lapin SL, Seale-Hawkins C, Link J, Wheeler TM. The mechanisms and prognostic significance of seminal vesicle involvement by prostate cancer. Am J Surg Pathol 1993; 17:1252-1261.

131. John H, Hauri D. Seminal vesicle-sparing radical prostatectomy: a novel concept to restore early urinary continence. Urology 2000 ; 55:820-824.

132. Ravery V, Chastang C, Toublanc M, Boccon-Gibod L, Delmas V. Percentage of cancer on biopsy cores accurately predicts extracapsular extension and biochemical relapse after radical prostatectomy for T1-T2 prostate cancer. Eur Urol 2000; 37:449-455.

133. Cagiannos I, Karakiewicz P, Eastham JA, et al. A preoperative nomogram identifying decreased risk of positive pelvic lymph nodes in patients with prostate cancer. J Urol 2003; 170:1798-1803.

134. Gervasi LA, Mata J, Easley JD, et al. Prognostic significance of lymph nodal metastases in prostate cancer. J Urol 1989; 142:332-336.

135. Parker CC, Dearnaley DP. Radical radiotherapy for prostate cancer. Cancer Treat Rev 2003; 29:161-169.

136. Naya $Y$, Fritsche HA, Cheli CD, et al. Volume indexes of total, free, and complexed prostate-specific antigen enhance prediction of extraprostatic disease extension in men with nonpalpable prostate cancer. Urology 2003; 62:1058-1062.

137. Harisinghani MG, Barentsz J, Hahn PF, et al. Noninvasive detection of clinically occult lymph-node metastases in prostate cancer. N Eng J Med 2003; 348:2491-2499.

138. Borley N, Fabrin K, Sriprasad S, et al. Laparoscopic pelvic lymph node dissection allows significantly more accurate staging in "high-risk" prostate cancer compared to MRI or CT. Scand J Urol Nephrol 2003; 37:382-386.

139. Bellin MF, Roy C, Kinkel K, et al. Lymph node metastases: safety and effectiveness of MR imaging with ultrasmall superparamagnetic iron oxide particles--initial clinical experience. Radiology 1998; 207:799-808.

140. Young $\mathrm{HH}$. The early diagnosis and radical cure of carcinoma of the prostate. Being a study of 40 cases and presentation of a radical operation which was carried out in four cases. 1905. J Urol 2002; 168:914-921.

141. Walsh PC, Donker PJ. Impotence following radical prostatectomy: insight into etiology and prevention. J Urol 1982; $128: 492-497$.

142. Abbas F, Scardino PT. Why neoadjuvant androgen deprivation prior to radical prostatectomy is unnecessary. Urol Clin North Am 1996; 23:587-604

143. Kim ED, Nath R, Slawin KM, Kadmon D, Miles BJ, Scardino PT. Bilateral nerve grafting during radical retropubic prostatectomy: extended follow-up. Urology 2001; 58:983-987.

144. Kim ED, Nath R, Kadmon D, et al. Bilateral nerve graft during radical retropubic prostatectomy: 1-year followup. J Urol 2001; 165:19501956.

145. Kim ED, Scardino PT, Hampel O, Mills NL, Wheeler TM, Nath RK. Interposition of sural nerve restores function of cavernous nerves resected during radical prostatectomy. J Urol 1999; 161:188-192.

146. Karakiewicz PI, Tanguay S, Kattan MW, Elhilali MM, Aprikian AG. Erectile and urinary dysfunction after radical prostatectomy for prostate cancer in Quebec: a population-based study of 2415 men. Eur Urol 2004; 46:188-194.

147. Rabbani F, Stapleton AM, Kattan MW, Wheeler TM, Scardino PT. Factors predicting recovery of erections after radical prostatectomy. J Urol 2000; 164:1929-1934.

148. Rosen MA, Goldstone L, Lapin S, Wheeler T, Scardino PT. Frequency and location of extracapsular extension and positive surgical margins in radical prostatectomy specimens. J Urol 1992; 148:331-337.

149. Eastham JA, Scardino PT. Early diagnosis and treatment of prostate cancer. Dis Mon 2001; 47:421-459.

150. Sofer M, Hamilton-Nelson KL, Schlesselman JJ, Soloway MS. Risk of positive margins and biochemical recurrence in relation to nervesparing radical prostatectomy. J Clin Oncol 2002; 20:1853-1858

151. Sofer M, Hamilton-Nelson KL, Civantos F, Soloway MS. Positive surgical margins after radical retropubic prostatectomy: the influence of site and number on progression. J Urol 2002; 167:2453-2456.

152. Aranha O, Vaishampayan U.PSA relapse prostate cancer: the importance of tailored therapy. Urol Oncol 2004; 22:62-69.

153. Freedland SJ, Humphreys EB, Mangold LA, et al. Risk of prostate cancer-specific mortality following biochemical recurrence after radical prostatectomy. Jama 2005; 294:433-439.

154. Han M, Partin AW, Zahurak M, Piantadosi S, Epstein J, Walsh PC. Biochemical (prostate specific antigen) recurrence probability following radical prostatectomy for clinically localized prostate cancer. J Urol 2003; 169:517-523.

155. Tewari A, Gamito EJ, Crawford ED, Menon M. Biochemical recurrence and survival prediction models for the management of clinically localized prostate cancer. Clin Prostate Cancer 2004; 2:220-227.

156. Moul JW. Biochemical recurrence of prostate cancer. Curr Probl Cancer 2003; 27:243-272.

157. Stephenson AJ, Scardino PT, Eastham JA, et al. Preoperative nomogram predicting the 10-year probability of prostate cancer recurrence after radical prostatectomy. J Natl Cancer Inst 2006; 98:715-717.

158. Stephenson AJ, Scardino PT, Eastham JA, et al. Postoperative nomogram predicting the 10-year probability of prostate cancer recurrence after radical prostatectomy. J Clin Oncol 2005; 23:7005-7012.

159. Graefen M, Karakiewicz PI, Cagiannos I, et al. A validation of two preoperative nomograms predicting recurrence following radical prostatectomy in a cohort of European men. Urol Oncol 2002; 7:141-146.

160. Hricak H, Schoder H, Pucar D, et al. Advances in imaging in the postoperative patient with a rising prostate-specific antigen level. Semin Oncol 2003; 30:616-634

161. Albertsen PC, Fryback DG, Storer BE, Kolon TF, Fine J. Long-term survival among men with conservatively treated localized prostate cancer. Jama 1995; 274:626-631

162. Fryback DG, Albertsen PC, Storer BE. Prostatectomy and survival among men with clinically localized prostate cancer. Jama 1996; 276:1723-1724; author reply 1724.

163. Gleason DF. Classification of prostatic carcinomas. Cancer Chemother Rep 1966; 50:125-128.

164. Gleason DF, Mellinger GT. Prediction of prognosis for prostatic adenocarcinoma by combined histological grading and clinical staging. J Urol 1974: 111:58-64.

165. Epstein J, Algaba F, Allsbrook Jea. Acinar adenocarcinoma. In: Eble JN, Sauter G, Epstein Jl, et al, eds. World Health Organization Classification of Tumours. Pathology \& Genetics: Tumours of the Urinary System and Male Genital Organs. . Lyon, France: IARC Press, 2004 2004:179-184. 
166. Epstein Jl, Allsbrook WC, Jr., Amin MB, Egevad LL. The 2005 International Society of Urological Pathology (ISUP) Consensus Conference on Gleason Grading of Prostatic Carcinoma. Am J Surg Pathol 2005; 29:1228-1242.

167. Epstein J. What's new in prostate cancer disease assessment in 2006? Curr Opin Urol 2006; 16:146-151.

168. Epstein Jl, Allsbrook WC, Jr., Amin MB, Egevad LL. Update on the Gleason grading system for prostate cancer: results of an international consensus conference of urologic pathologists. Adv Anat Pathol 2006; 13:57-59.

169. Wang L, Hussain SM, Heerebeek BV, Pattynama PM, Vogel MW, Krestin GP. Postprocessing of Dynamic Gadolinium-enhanced MRI Exams of the upper Abdominal Organs: Explanation and Potential Clinical Application of Color-coded Qualitative and Quantitative Analysis. Radiology 2000; $217(P): 635$.

170. Radjenovic A, Ridgway JP, Smith MA. A method for pharmacokinetic modelling of dynamic contrast enhanced MRI studies of rapidly enhancing lesions acquired in a clinical setting. Phys Med Biol 2006; 51:N187-197.

171. Port RE, Knopp MV, Hoffmann U, Milker-Zabel S, Brix G. Multicompartment analysis of gadolinium chelate kinetics: blood-tissue exchange in mammary tumors as monitored by dynamic MR imaging. J Magn Reson Imaging 1999; 10:233-241.

172. Krestin GP, Lorenz R, Steinbrich W. [Magnetic resonance tomography of adrenal gland tumors. Detection and differentiation using fast gradient echo sequences and dynamic contrast media studies]. Radiologe 1990; 30:228-234.

173. Krestin GP, Freidmann G, Fishbach R, Neufang KF, Allolio B. Evaluation of adrenal masses in oncologic patients: dynamic contrastenhanced MR vs CT. J Comput Assist Tomogr 1991; 15:104-110.

174. Kuhl CK, Mielcareck P, Klaschik S, et al. Dynamic breast MR imaging: are signal intensity time course data useful for differential diagnosis of enhancing lesions? Radiology 1999; 211:101-110.

175. Kim JK, Hong SS, Choi YJ, et al. Wash-in rate on the basis of dynamic contrast-enhanced MRI: usefulness for prostate cancer detection and localization. J Magn Reson Imaging 2005; 22:639-646.

176. van Vliet M, van Dijke CF, Wielopolski PA, et al. MR angiography of tumor-related vasculature: from the clinic to the micro-environment. Radiographics 2005; 25 Suppl 1:S85-97; discussion S97-88.

177. Preda A, van Vliet M, Krestin GP, Brasch RC, van Dijke CF. Magnetic resonance macromolecular agents for monitoring tumor microvessels and angiogenesis inhibition. Invest Radiol 2006; 41:325-331.

178. Tofts PS, Brix G, Buckley DL, et al. Estimating kinetic parameters from dynamic contrast-enhanced T(1)-weighted MRI of a diffusable tracer: standardized quantities and symbols. J Magn Reson Imaging 1999; 10:223-232.

179. Verstraete KL, Vanzieleghem B, De Deene Y, et al. Static, dynamic and first-pass MR imaging of musculoskeletal lesions using gadodiamide injection. Acta Radiol 1995; 36:27-36

180. Verstraete KL, De Deene Y, Roels H, Dierick A, Uyttendaele D, Kunnen M. Benign and malignant musculoskeletal lesions: dynamic contrastenhanced MR imaging--parametric "first-pass" images depict tissue vascularization and perfusion. Radiology 1994; 192:835-843.

181. Brown G, Macvicar DA, Ayton V, Husband JE. The role of intravenous contrast enhancement in magnetic resonance imaging of prostatic carcinoma. Clin Radiol 1995; 50:601-606

182. Jager GJ, Ruijter ET, van de Kaa CA, et al. Dynamic TurboFLASH subtraction technique for contrast-enhanced MR imaging of the prostate: correlation with histopathologic results. Radiology 1997; 203:645-652.

183. Padhani AR, Gapinski CJ, Macvicar DA, et al. Dynamic contrast enhanced MRI of prostate cancer: correlation with morphology and tumour stage, histological grade and PSA. Clin Radiol 2000; 55:99-109.

184. Ogura K, Maekawa S, Okubo K, et al. Dynamic endorectal magnetic resonance imaging for local staging and detection of neurovascular bundle involvement of prostate cancer: correlation with histopathologic results. Urology 2001; 57:721-726.

185. Rinck PA, Torheim G, Lombardi M. Image postprocessing and contrast agents in clinical MR imaging--an introductory overview. Acta Radiol Suppl 1997; 412:7-19.

186. Parker GJ, Tofts PS. Pharmacokinetic analysis of neoplasms using contrast-enhanced dynamic magnetic resonance imaging. Top Magn Reson Imaging 1999; 10:130-142.

187. Hamm B, Mahfouz AE, Taupitz M, et al. Liver metastases: improved detection with dynamic gadolinium-enhanced MR imaging? Radiology 1997; 202:677-682.

188. Chen B, Pogue BW, Luna JM, Hardman RL, Hoopes PJ, Hasan T. Tumor vascular permeabilization by vascular-targeting photosensitization: effects, mechanism, and therapeutic implications. Clin Cancer Res 2006; 12:917-923.

189. Rouviere O, Raudrant A, Ecochard R, et al. Characterization of time-enhancement curves of benign and malignant prostate tissue at dynamic MR imaging. Eur Radiol 2003; 13:931-942.

190. Schlemmer HP, Merkle J, Grobholz R, et al. Can pre-operative contrast-enhanced dynamic MR imaging for prostate cancer predict microvessel density in prostatectomy specimens? Eur Radiol 2004; 14:309-317.

191. van Dorsten FA, van der Graaf M, Engelbrecht MR, et al. Combined quantitative dynamic contrast-enhanced MR imaging and (1)H MR spectroscopic imaging of human prostate cancer. J Magn Reson Imaging 2004; 20:279-287.

192. Buckley DL, Roberts C, Parker GJ, Logue JP, Hutchinson CE. Prostate cancer: evaluation of vascular characteristics with dynamic contrastenhanced T1-weighted MR imaging--initial experience. Radiology 2004; 233:709-715

193. Preziosi P, Orlacchio A, Di Giambattista G, et al. Enhancement patterns of prostate cancer in dynamic MRI. Eur Radiol 2003; 13:925-930.

194. Huisman HJ, Engelbrecht MR, Barentsz JO. Accurate estimation of pharmacokinetic contrast-enhanced dynamic MRI parameters of the prostate. J Magn Reson Imaging 2001; 13:607-614.

195. Ito H, Kamoi K, Yokoyama K, Yamada K, Nishimura T. Visualization of prostate cancer using dynamic contrast-enhanced MRI: comparison with transrectal power Doppler ultrasound. Br J Radiol 2003; 76:617-624.

196. Engelbrecht MR, Huisman HJ, Laheij RJ, Jager GJ, van Leenders GJ, Hulsbergen-Van, De Kaa CA dIRJ, Blickman JG, Barentsz JO. Discrimination of prostate cancer from normal peripheral zone and central gland tissue by using dynamic contrast-enhanced MR imaging. Radiology 2003; 229:248-254.

197. Kiessling F, Lichy M, Grobholz R, et al. Simple models improve the discrimination of prostate cancers from the peripheral gland by T1weighted dynamic MRI. Eur Radiol 2004; 14:1793-1801.

198. Barentsz JO, Engelbrecht M, Jager GJ, et al. Fast dynamic gadolinium-enhanced MR imaging of urinary bladder and prostate cancer. J Magn Reson Imaging 1999; 10:295-304.

199. Oyen RH. Dynamic contrast-enhanced MRI of the prostate: is this the way to proceed for characterization of prostatic carcinoma? Eur Radiol 2003; 13:921-924.

200. Hanahan D, Folkman J. Patterns and emerging mechanisms of the angiogenic switch during tumorigenesis. Cell 1996; 86:353-364.

201. Li WW. Tumor angiogenesis: molecular pathology, therapeutic targeting, and imaging. Acad Radiol 2000; 7:800-811.

202. Shames DM, Kuwatsuru R, Vexler V, Muhler A, Brasch RC. Measurement of capillary permeability to macromolecules by dynamic magnetic resonance imaging: a quantitative noninvasive technique. Magn Reson Med 1993; 29:616-622. 
203. Jain RK. Barriers to drug delivery in solid tumors. Sci Am 1994; 271:58-65.

204. Brasch R, Turetschek K. MRI characterization of tumors and grading angiogenesis using macromolecular contrast media: status report. Eur J Radiol 2000; 34:148-155.

205. Roberts C, Issa B, Stone A, Jackson A, Waterton JC, Parker GJ. Comparative study into the robustness of compartmental modeling and model-free analysis in DCE-MRI studies. J Magn Reson Imaging 2006; 23:554-563.

206. Pham CD, Roberts TP, van Bruggen N, et al. Magnetic resonance imaging detects suppression of tumor vascular permeability after administration of antibody to vascular endothelial growth factor. Cancer Invest 1998; 16:225-230.

207. Ito K. Hepatocellular carcinoma: conventional MRI findings including gadolinium-enhanced dynamic imaging. Eur J Radiol 2006; 58:186199.

208. Rouviere O, Hartman RP, Lyonnet D. Prostate MR imaging at high-field strength: evolution or revolution? Eur Radiol 2006; 16:276-284.

209. Hricak H. New horizons in genitourinary oncologic imaging. Abdom Imaging 2006; 31:182-187.

210. Hussain SM, Wielopolski PA, Martin DR. Abdominal Magnetic Resonance Imaging at 3.0 T: Problem or a Promise for the Future? Top Magn Reson Imaging 2005; 16:325-335

211. Beyersdorff D, Taymoorian K, Knosel T, et al. MRI of prostate cancer at 1.5 and 3.0 T: comparison of image quality in tumor detection and staging. AJR Am J Roentgenol 2005; 185:1214-1220.

212. Futterer JJ, Heijmink SW, Scheenen TW, et al. Prostate cancer: local staging at 3-T endorectal MR imaging--early experience. Radiology 2006; 238:184-191.

213. Futterer JJ, Scheenen TW, Huisman HJ, et al. Initial experience of 3 tesla endorectal coil magnetic resonance imaging and $1 \mathrm{H}$-spectroscopic imaging of the prostate. Invest Radiol 2004; 39:671-680.

214. Schick F. Whole-body MRI at high field: technical limits and clinical potential. Eur Radiol 2005; 15:946-959.

215. Takahashi M, Uematsu H, Hatabu H. MR imaging at high magnetic fields. Eur J Radiol 2003; 46:45-52.

216. Uematsu H, Takahashi M, Dougherty L, Hatabu H. High field body MR imaging: preliminary experiences. Clin Imaging $2004 ; 28: 159-162$.

217. Kim HW, Buckley DL, Peterson DM, et al. In vivo prostate magnetic resonance imaging and magnetic resonance spectroscopy at 3 Tesla using a transceive pelvic phased array coil: preliminary results. Invest Radiol 2003; 38:443-451.

218. de Bazelaire CM, Duhamel GD, Rofsky NM, Alsop DC. MR imaging relaxation times of abdominal and pelvic tissues measured in vivo at 3.0 T: preliminary results. Radiology 2004; 230:652-659.

219. Sosna J, Rofsky NM, Gaston SM, DeWolf WC, Lenkinski RE. Determinations of prostate volume at 3-Tesla using an external phased array coil: comparison to pathologic specimens. Acad Radiol 2003; 10:846-853.

220. Sosna J, Pedrosa I, Dewolf WC, Mahallati H, Lenkinski RE, Rofsky NM. MR imaging of the prostate at 3 Tesla: comparison of an external phased-array coil to imaging with an endorectal coil at 1.5 Tesla. Acad Radiol 2004; 11:857-862.

221. Bloch BN, Rofsky NM, Baroni RH, Marquis RP, Pedrosa I, Lenkinski RE. 3 Tesla magnetic resonance imaging of the prostate with combined pelvic phased-array and endorectal coils; Initial experience(1). Acad Radiol 2004; 11:863-867. 


\section{Cripteneral Summary and Conclusions}




\section{General Summary and Conclusions}

$\mathbf{T}$

The high incidence of prostate cancer, combined with downstaging at diagnosis and the slow natural progression of the disease, has made its management a complex and controversial issue. Endorectal MRI is emerging as the most accurate imaging modality for the local anatomic assessment of prostate cancer. This dissertation assesses the value of current State-of-the-Art endorectal MRI in clinical practice and discusses the promise of the modality for improving prostate cancer management.

MR images allow noninvasive assessment of the local extent of prostate cancer (including organ-confinement, extracapsular extension, seminal vesicle invasion and lymph node metastasis) and can provide an indication of tumor aggressiveness based on signal intensity; thus MRI can assist in local staging and assessment of recurrence-free probabilities while providing surgeons with a visual road-map for optimal treatment planning. MRSI of the prostate depicts the altered metabolism associated with prostate cancer. The addition of $1 \mathrm{H}$ MRSI to MRI has further improved the accuracy of MR imaging in prostate cancer staging. It may become possible to use MRI/1H MRSI to achieve more precise stratification of patients in clinical trials, to monitor the progress of patients who select watchful waiting or minimally aggressive cancer therapies, and to guide and assess emerging local prostate cancer therapies. The second part of this dissertation illustrates the potential clinical application of color-coded parameter imaging derived from postprocessing of dynamic multiphasic gadolinium-enhanced MRI (DMGE-MRI) of abdominal tumors. With more refined techniques, such as high-field imaging, and with more experienced readers and more uniform image interpretation, the MR imaging-MR spectroscopy approach will have a tremendous capacity to improve patient care.

Chapter 2 shows that MR findings (from endorectal MRI or combined endorectal MRI/MRSI) contribute significant incremental value to clinical staging nomograms in the prediction of organ-confined prostate cancer (OCPC) in all risk groups (low, intermediate, and high). The incorporation of endorectal MR imaging into future staging nomograms for the prediction of OCPC may therefore be warranted, particularly since clinical staging in the current staging nomograms is based only on digital rectal examination. Moreover, unlike MRI, the staging nomograms cannot assist in the localization of ECE, which is critical for optimal treatment planning. It is worth noting that in the study, the accuracy of radiologists' predictions of OCPC was higher in the combined MR group (MRI+MRSI) than in the endorectal MR imaging.

Chapter 3 indicates that endorectal MR imaging findings are significant presurgical predictors of extracapsular extension (ECE) in patients with prostate cancer and add incremental value to clinical variables. At univariate analysis, all variables were associated with ECE. At ROC univariate analysis, endorectal MR imaging findings had the largest area under the ROC curve. At multivariate analysis, serum PSA level, greatest percentage of cancer in all core biopsy specimens, and endorectal MR imaging findings were predictors of ECE. A model containing endorectal MR imaging findings had a significantly larger area under the ROC curve than a model containing only clinical variables. In addition, endorectal MR imaging findings are spatially localized, and therefore, unlike clinical variables, they have the potential to allow tailored treatment modifications.

Chapter 4 demonstrates that endorectal MR imaging findings can be significant predictors for ECE in patients with prostate cancer, after controlling for PSA level, Gleason score, greatest percentage of cancer in all core biopsy specimens, percentage of cancer-positive core specimens in all core biopsy specimens, PNI, and clinical stage of tumor. A comparison of the ROC curves drawn from the results of readings performed by genitourinary MR imaging radiologists and general body MR imaging radiologists showed that endorectal MR imaging findings added value to all other predictor variables only when MR images were interpreted by genitourinary MR imaging radiologists. In the genitourinary radiologist group endorectal MR imaging findings displayed a combination of sensitivity and specificity that was superior to that of all other predictors tested. In the general body radiologist group, however, the combination of sensitivity and specificity of endorectal MR imaging findings was similar to that of the clinical predictors. Advances in technology and in the expertise of radiologists dedicated to the genitourinary field suggest that endorectal MR imaging could play an increasingly useful role in the treatment of patients with prostate cancer. 
Chapter 5 shows that endorectal MRI findings can contribute significant incremental value to the Kattan nomogram for predicting SVI. On both univariate and multivariate analysis, endorectal MRI findings were a significant presurgical predictor of seminal vesicle invasion.

Chapter 6 shows that a PACS cross-referencing tool allows radiologists to more accurately interpret prostate MR imaging, improving prostate cancer tumor staging by MRI. In the detection of ECE and SVI, radiologists performed substantially better when using MRI with PACS cross-referencing rather than MRI alone. PACS cross-referencing is particularly helpful in displaying the junction of the seminal vesicles and the central zone of the prostate.

Chapter 7 describes a study showing that incorporation of the Partin nomogram results with MRI findings regarding both extracapsular extension and seminal vesicle invasion improves the MR prediction of LNM in patients with prostate cancer. The study confirmed that MRI has a high negative predictive value and an exceptionally high specificity in the prediction of LNM compared with clinical and histological variables. As MRI also provides anatomical information that is useful in treatment planning, it could potentially be used along with the Partin nomogram to determine whether additional imaging with lymphotropic superparamagnetic nanoparticles is indicated.

Chapter 8 shows that review of preoperative MRI findings significantly improves the surgeon's decision to preserve or resect the NVB(s) during radical prostatectomy. Preoperative MRI improved surgical planning in high-risk patients and provided appropriate reassurance for preserving the NVB(s) in other patients. The strength of MRI for low-risk and intermediate-risk patients lies in a high negative predictive value (i.e., demonstration of the absence of tumor in the region of interest).

Chapter 9 shows that the addition of MRI findings significantly improves the accuracy of the Kattan pre-operative nomogram in predicting postoperatively determined recurrence-free probabilities in the total study cohort and in a subset of patients (PSA $\geq 10$, Gleason score $\geq 7$ on biopsy or clinical stage T2) at high risk for biochemical recurrence.

Chapter $\mathbf{1 0}$ discusses a study demonstrating that MRI has potential in the noninvasive assessment of prostate cancer biological aggressiveness. First, there is a significant correlation between prostate cancer Gleason grade and tumor-to-muscle signal intensity ratio on T2-weighted MR images; a higher Gleason grade is associated with a lower tumor-to-muscle signal intensity ratio. Second, on T2-weighted MR images, Gleason grade 3 cancer in the transition zone has a significantly lower tumor-to-muscle signal intensity ratio than Gleason grade 3 cancer in the peripheral zone of the prostate.

Chapter 11 is restricted to current reviews of major topics in radiology and brief case reports on postprocessing of dynamic multiphasic gadolinium-enhanced MRI (DMGE-MRI) of the abdominal tumors. Post-processing of dynamic multiphasic gadolinium-enhanced MRI (DMGE-MRI) of the abdomen allows the generation of color-coded parameter images and time-intensity curves that provide new opportunities for research and for improving the detection, staging and therapeutic monitoring of disease and the development of anti-tumor drugs. First the color-coded parameter images and time-intensity curves contribute to a better understanding of tumor enhancement patterns and angiogenesis, and hence may result in improved quantitative characterization of tumors. Second, the mechanisms behind the differential signal enhancement of dynamic multiphasic gadolinium-enhanced MRI (DMGE-MRI) are thought to include differences in tumor perfusion and the levels of tumor capillary wall permeability and hydrostatic pressure. Third, the time-intensity curves and only a small number of parameters need to be displayed as color-coded parameter images on a workstation. These robust parameters include: 1) relative enhancement; 2) wash-in rate; 3 ) wash-out rate; 4) brevity of enhancement.

In Chapter 12, the results of all the studies mentioned above are discussed in relation to one another and to the current literature. Recommendations for clinical practice are made. 


\section{Conclusions}

\section{The following overall conclusions can be made:}

1. In patients with prostate cancer, endorectal MR imaging non-invasively improves pretreatment staging and treatment planning and the evaluation of recurrence-free probabilities; it can also provide an indication of cancer aggressiveness.

2. The incorporation of endorectal MR findings into future nomograms for the prediction of prostate cancer stage and freedom from biochemical recurrence is warranted.

3. Advances in technology, such as a PACS cross-referencing tool, and in the expertise of radiologists dedicated to the genitourinary field suggest that endorectal MR imaging can play an increasingly useful role in prostate cancer management.

4. Information from preoperative MR imaging allows the surgeon to significantly refine the surgical plan, maximizing the preservation of periprostatic tissues (important for recovery of urinary and sexual function)

5. Inclusion of time-intensity curves and color-coded images as part of the routine abdominal MR imaging work-up protocol facilitates the diagnostic work-up of disease for detection, staging, and monitoring of anti-tumor therapy. 
13 


\section{Samenvatting}

D e hoge incidentie van prostaatkanker in combinatie met een vroegere opsporing, downstaging ten tijde van de diagnose en de langzame natuurlijke voortgang van deze ziekte heeft de beheersing ervan tot een complexe en controversiële kwestie gemaakt. De meest nauwkeurige beeldvormende modaliteit voor de lokale anatomische beoordeling van prostaatkanker blijkt MRI te zijn.

Dit proefschrift presenteert de huidige toepassing van geavanceerde MRI in de klinische praktijk en bespreekt de verwachtingen van deze modaliteit voor een betere beheersing van prostaatkanker. MR-beelden maken een nietinvasieve beoordeling van de lokale omvang (met inbegrip van OC, ECE, SVI en LNM) en verandering van de signaalintensiteit mogelijk, waardoor deze behulpzaam kunnen zijn bij de lokale stadiëring en bij het bepalen van de kans op agressieve tumoren en de kans dat tumoren niet zullen terugkeren, terwijl het artsen voorziet van een visuele routekaart voor de behandelingsplanning om ervan verzekerd te zijn dat de kanker in zijn geheel wordt verwijderd en dat kanker op de lange termijn beter kan worden bestreden zonder dat de kritieke zenuwen en de sfincter die de geslachts- en urinefunctie regelen verwijderd hoeven te worden. MRSI van de prostaat geeft een beeld van het veranderde metabolisme dat verband houdt met prostaatkanker. Door $1 \mathrm{H}$ MRSI toe te voegen aan MRI is de nauwkeurigheid van MR bij de stadiëring van prostaatkanker nog verder verbeterd. Wellicht wordt het mogelijk om door middel van MRI/1H MRSI in klinische proeven een preciezere stratificatie van patiënten te bewerkstelligen, om de voortgang te bewaken bij patiënten die kiezen voor oplettend afwachten of minimaal agressieve kankertherapieën, en om nieuwe lokale prostaatkankertherapieën te begeleiden en te beoordelen. Het tweede deel van het proefschrift beschrijft de potentiële klinische toepassing van kleurgecodeerde parameterbeeldvorming die is afgeleid van de nabewerking van dynamische meerfasige met gadolinium verbeterde MRI (DMGE-MRI) van abdominale tumoren: prostaatkanker als één van paradigma's. Met meer verfijnde technieken, zoals beeldvorming met hoge veldsterkte, meer ervaren lezers en een meer uniforme beeldinterpretatie, zal de benadering van MRI en MR-spectroscopie belangrijke kansen bieden om de patiëntenzorg te verbeteren.

In Hoofdstuk 2 wordt aangetoond dat MR-bevindingen (op basis van endorectale MRI of een combinatie van endorectale MRI en MR-spectografische beeldvorming) aanzienlijke incrementele waarde toevoegen de klinische stadiëringsnomogrammen bij de predictie van orgaanbeperkte prostaatkanker. Hierdoor kan worden gewaarborgd dat endorectale MRI in de toekomst deel zal uitmaken van stadiëringsnomogrammen voor de predictie van orgaanbeperkte prostaatkanker. Ten eerste is er, ondanks het krachtige predictievermogen en de kosteneffectiviteit van de stadiëringsnomogrammen, ruimte voor verbetering van de nauwkeurigheid van de predictie, vooral omdat de klinische stadiëring bij de stadiëringsnomogrammen uitsluitend gebaseerd is op digitaal rectaal onderzoek. Bovendien kunnen de stadiëringsnomogrammen niet gebruikt worden voor het lokaliseren van ECE, wat voor een optimale behandelingsplanning essentieel is. Ten tweede is het oppervlak onder de ROC-curve door de toevoeging van MR-bevindingen zowel bij de groep met een laag risico als bij de groepen met een middelmatig en hoog risico aanzienlijk groter geworden. Ten derde is de nauwkeurigheid van de predictie van orgaanbeperkte prostaatkanker door radiologen bij de groep met een combinatie van MR (MRI + MRSI) groter dan bij de groep met endorectale MRI.

In Hoofdstuk 3 wordt aangegeven dat de bevindingen op basis van endorectale MRI bij patiënten met prostaatkanker belangrijke prechirurgische predictoren van ECE zijn en incrementele waarde toevoegen aan klinische variabelen. Ten eerste werden bij de univariate analyse alle variabelen in verband gebracht met ECE. Bij de univariate analyse van de ROC bleek het oppervlak onder de ROC-curve bij de bevindingen op basis van endorectale MRI het grootst te zijn. Bij de multivariate analyse waren het PSA-gehalte in het serum, het percentage kanker bij alle kritieke biopten en de bevindingen op basis van endorectale MRI predictoren van ECE. Een model met daarin de bevindingen op basis van endorectale MRI heeft onder de ROC-curve een aanzienlijk groter oppervlak dan een model met uitsluitend klinische variabelen. Bovendien zijn de bevindingen op basis van endorectale MRI ruimtelijk gelokaliseerd en bieden deze hierdoor in tegenstelling tot klinische variabelen mogelijkheden voor maatwerk in de behandeling.

In Hoofdstuk 4 wordt aangetoond dat de bevindingen op basis van endorectale MRI bij patiënten met prostaatkanker belangrijke predictoren van ECE kunnen zijn na rekening te hebben gehouden met het PSA-gehalte, de Gleason-score, het hoogste percentage kanker in alle kernbiopten, het percentage kankerpositieve kernbiopten bij alle kernbiopten, PNI en de klinische stage van de tumor. Uit een vergelijking van de ROC-curves, getekend op basis 
van de resultaten van de metingen die zijn verricht door radiologen met behulp van MRI van de genitourinaire tractus en door radiologen met behulp van MRI van het lichaam in zijn algemeenheid, is gebleken dat de bevindingen op basis van endorectale MRI alleen waarde toevoegen aan alle andere predictievariabelen wanneer de MRbeelden worden geïnterpreteerd door de radiologen die gewerkt hadden met MRI van de genitourinaire tractus. Ten eerste lieten de bevindingen op basis van endorectale MRI door de groep radiologen van de genitourinaire tractus een combinatie van gevoeligheid en specificiteit zien die beter was dan bij alle andere geteste predictoren. Bij de groep radiologen die gewerkt hadden met MRI van het lichaam in zijn algemeenheid, leek de combinatie van gevoeligheid en specificiteit van de bevindingen op basis van endorectale MRI echter op die van de klinische predictoren. Ten tweede voegen de bevindingen op basis van endorectale MRI aanzienlijke waarde toe bij de diagnose van ECE bij patiënten met prostaatkanker wanneer MR-beelden worden geïnterpreteerd door radiologen met ervaring in MRI van de prostaat. De ontwikkelingen in de technologie en in de deskundigheid van radiologen die gespecialiseerd zijn in de genitourinaire tractus wijzen erop dat de endorectale MRI een steeds nuttigere rol zou kunnen spelen bij de behandeling van patiënten met prostaatkanker.

In Hoofdstuk 5 wordt aangegeven dat de bevindingen op basis van endorectale MRI aanzienlijke incrementele waarde kunnen toevoegen aan het Kattan-nomogram voor de predictie van SVI. Hoewel verdere bevestigende onderzoeken van meerdere centra nuttig zouden zijn, gaan wij ervan uit dat MRI een belangrijke rol kan spelen bij de aan behandeling voorafgaande niet-invasieve evaluatie van prostaatkanker, met inbegrip van de predictie van SVI. Ten eerste zijn de bevindingen op basis van endorectale MRI na univariate en multivariate analyse een belangrijke prechirurgische predictor van aantasting van de zaadblaasjes (seminal vesicle-invasie). Ten tweede wordt er door het toevoegen van de bevindingen op basis van endorectale MRI aanzienlijke incrementele waarde toegevoegd aan het Kattan-nomogram voor de predictie van SVI.

Hoofdstuk 6 beschrijft het PACS-referentiehulpmiddel dat radiologen in staat stelt om de MRI van de prostaat nauwkeuriger te interpreteren waardoor de stadiëring van prostaatkankertumoren door MRI aanzienlijk wordt verbeterd. Bij het opsporen van ECE en SVI presteerden radiologen bij het toepassen van MRI met PACS-referentie aanzienlijk beter dan met alleen MRI. PACS-referentie is vooral nuttig voor beeldvorming van de verbinding tussen de zaadblaasjes en de centrale zone van de prostaat.

In Hoofdstuk 7 wordt nader ingegaan op het feit dat de MR-predictie van LNM bij patiënten met prostaatkanker wordt verbeterd wanneer de resultaten van Partin-nomogrammen en de bevindingen van MRI betreffende extracapsulaire extensie en seminal vesicle-invasie mede in beschouwing worden genomen. Ten tweede zou een combinatie van endorectale en gefaseerde reeks MRI's, aangevuld met de Partin-nomogrammen, gebruikt kunnen worden om te bepalen of aanvullende beeldvorming met lymfotropische superparamagnetische nanodeeltjes geïndiceerd is. Ten derde wordt bevestigd dat erMRI in vergelijking met klinische en histologische variabelen een hoge negatieve predictieve waarde en een uitzonderlijk hoge specificiteit heeft bij de predictie van LNM. Ten vierde verschaft erMRI als zodanig anatomische informatie die nuttig is voor de behandelingsplanning.

In Hoofdstuk 8 wordt de incrementele waarde van endorectale magnetische resonantiebeeldvorming (eMRI) voor deze voorbehandelingsplanning bepaald. Door een preoperatieve MRI kan de chirurg veel beter bepalen of de NVB moet worden behouden of operatief moet worden verwijderd tijdens radicale prostatectomie, en daardoor aanvullende kritieke informatie verschaffen zodat de behandeling optimaal kan worden afgestemd op de patiënt. Ten eerste leidde eMRI voorafgaand aan RP bij patiënten met een hoog risico tot een betere chirurgische planning, en zorgde deze bij de andere patiënten voor de nodige geruststelling omdat de NVB kon worden behouden. Bij de groep met een hoog risico heeft MRI incrementele waarde bij de klinische beoordeling als aanvulling op het Partinstadiëringsnomogram, een vereenvoudigde klinische risicostratificatie (met gebruikmaking van het PSA-gehalte, de Gleason-score en het klinische ziektestadium) of het percentage positieve biopsiescores. Ten tweede hielp MRI bij de groepen met een laag en middelmatig risico om voor de nodige geruststelling te zorgen in die gevallen waarin behoud van de NVB in de chirurgische planning vóór de MRI de voorkeur genoot. Voor patiënten met een laag en middelmatig risico ligt de kracht van MRI in een hoge negatieve predictiewaarde (d.w.z. aantonen dat er in de betreffende regio geen tumoren aanwezig zijn).

In Hoofdstuk 9 wordt aangegeven dat de toevoeging van de bevindingen op basis van MRI tot een aanzienlijke verbetering leidt van de nauwkeurigheid van het preoperatieve Kattan-nomogram bij de predictie van postoperatief bepaalde waarschijnlijkheden van het niet-terugkeren van de kanker bij de totale onderzoekscohort en bij een 
deelgroep van patiënten (PSA $\geq 10$, Gleason-score $\geq 7$ na biopsie of klinisch stadium T2) met een hoog risico van biochemische herhaling.

In Hoofdstuk 10 wordt aangetoond dat MRI mogelijkheden biedt bij de niet-invasieve beoordeling van de agressiviteit van prostaatkanker. Ten eerste toont de signaalintensiteit van prostaatkanker op een T2-gewogen MRI een duidelijk verband met de pathologische Gleason-graad. De prostaattumor-spierverhouding op een T2-gewogen MRI toont een duidelijk negatief verband met de Gleason-score die is verkregen op basis van chirurgische pathologie. Ten tweede zijn de PZ-tumor-spiersignaalverhoudingen hoger dan die bij TZ.

Hoofdstuk 11 is beperkt tot actuele besprekingen van belangrijke onderwerpen op het gebied van radiologie en korte casusverslagen over de nabewerking van een dynamische meerfasige met gadolinium versterkte MRI (DMGEMRI) van de abdominale tumoren: prostaatkanker als één van paradigma's. De nabewerking van een dynamische meerfasige met gadolinium versterkte MRI (DMGE-MRI) van het abdomen maakt het mogelijk om kleurgecodeerde parameterbeelden en tijdsintensiteitscurves te genereren die nieuwe kansen bieden voor onderzoek en verbetering van de diagnostiek van ziekten voor de opsporing, stadiëring, therapeutische bewaking en ontwikkeling van medicijnen voor het bestrijden van tumoren. Ten eerste dragen de kleurgecodeerde parameterbeelden en tijdsintensiteitscurves op basis van de farmacokinetische modellering bij aan een beter begrip van tumorversterkingspatronen en angiogenese, en kan daardoor resulteren in een betere kwantitatieve karakterisering van tumoren. Ten tweede wordt gedacht dat de mechanismen achter de differentiaalsignaalversterking van dynamische, meerfasige met gadolinium versterkte MRI (DMGE-MRI) verschillen bevatten in tumorperfusie en de mate van hydrostatische druk en permeabiliteit van de capillaire wand van de tumor. Ten derde hoeven op een werkstation alleen de tijdsintensiteitscurves en slechts een klein aantal parameters te worden weergegeven als kleurgecodeerde parameterbeelden. Deze krachtige parameters zijn onder andere: 1) de relatieve versterking; 2) de instroomsnelheid (WIR); 3) de uitstroomsnelheid (WOR); 4) de korte duur van de versterking.

In Hoofdstuk 12 worden de resultaten van alle bovengenoemde onderzoeken besproken, zowel in relatie tot elkaar als in relatie tot de actuele literatuur. Er worden aanbevelingen voor de klinische praktijk gedaan. 
14 
Criptatement of Appreciation 


\section{Statement of Appreciation}

$\mathbf{F}$ irst of all, I would like to thank my thesis advisors, Prof. Dr. Gabriel P. Krestin at Erasmus MC, University Medical Center Rotterdam, the Netherlands, and Prof. Dr. Hedvig Hricak at Memorial Sloan-Kettering Cancer Center, the United States, for their constant guidance, encouragement and unconditional support throughout my Ph.D. research and for being excellent mentors and sharing their wisdom. Their perpetual pursuit of the highest level of perfection has inspired me to set high standards for myself.

I am deeply grateful to Prof. Dr. Wang Chengyuan at Tongji Medical University, China, and Prof. Dr. Li Guozhen at Beijing Hospital, China, for opening the window to western medical radiology and for being a constant inspiration. 我的硕士生导师同济医科大学王承缘教授: 您给我开启通向世界放射学的门。然后对我说:你是我的首屈一指 的学生留学荷兰 (后来美国)。您象我的妈妈一样叮嘱鼓励我在海外的工作, 学习和生活。在此, 谨向您致以 最诚挚的谢意。

Professor Dr. M.G. Myriam Hunink, thank you for being such a nice mentor regarding medical decision making. Professor Dr. S.M. Hussain, thank you for your valuable comment on the manuscript concerning tumor enhancement on dynamic MRI. Professor Dr. David M. Panicek, thank you for all the help and encouragement.

Special thanks are due to Ada Muellner, for being an excellent editor. Ada, you always kindly helped me with English, which is not my native language. I would like to thank Marja Thijse, Linda Everse, Adriaan Moelker, Indra van den Bos and Marc RW Engelbrecht, Bert V Heerebeek: Marja, thank you for communicating with me and Prof. Dr. Krestin in the past seven years and for having my summary translated into Dutch. Linda, thank you for your help with all the steps that had to be taken before the manuscript could be submitted based on Erasmus's Doctoral Regulations. Adriaan, we used to sit together and get along well. Thank you for all your helps including your PhD thesis and being paranimf in my thesis defense. Indra, thank you for revising our manuscript about tumor enhancement on dynamic MRI. Marc, thank you for your helpful comment on the manuscript concerning tumor enhancement on dynamic MRI. Bert, we used to work together for research projects and get along well. I am grateful to Ton Everaers for the lay-out and creative cover design.

Many thanks to my professors, doctors, and colleagues: Peter T. Scardino, Jason A. Koutcher, Jingbo Zhang, Kristen L. Zakian, Michael W. Kattan, Fergus V. Coakley, Oguz Akin, Amita Dave, Yousef Mazaheri Tehrani, Kentaro Kuroiwa, Christopher Riedl, Chinyere Onyebuchi, Darko Pucar, Chaya Moskowitz, Nicole M. Ishill, Peter M. T. Pattynama, Marc C. J. M. Kock, Mika W. Vogel, Mohamed Ouhlous, Jeroen J. Nikken, Edwin H. G. Oei, Andries Zwamborn, and others.

For their love, support, and encouragement, I want to express my deepest gratitude to my family: father Wang Xinchun, mother Chen Tiejv, wife Qiubai, and son Ming. 爸爸妈妈 (王心淳陈铁菊), 我获得博士学位了, 还是洋博士 学位。如果没有您们的精心养育和全力支持, 我不可能完成我的漫长的求学之路。秋白明明,我们仁有一个温 暖的家，有三张可爱的笑脸. Thanks to Qiubai for being paranimf in my thesis defense. I appreciate the Father's day gift from my four-year-old son: "Dear Daddy, The tree. A bird was standing next to a tree, and he dropped an egg. He dropped one more egg. Love, Ming Ming" 
15 


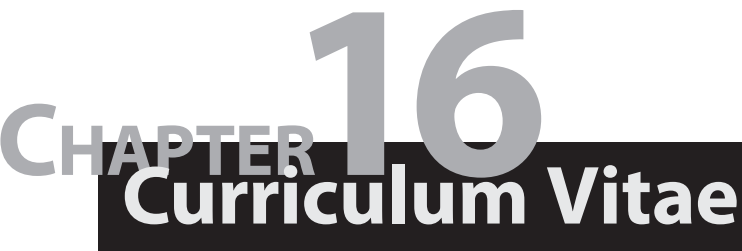




\section{Curriculum Vitae}

D r. Liang Wang was born in Lanzhou, China on June 27, 1966. His father is Xinchun Wang, a pioneering electrical and mechanical engineer since the 1950s. His mother is Tiejv Chen, a loving and caring financial accountant. Under their influence, he developed a strong interest in medical science. He obtained his medical degree (MD) in 1987 at the Medical College of Three Gorges University in China and completed a residency in medical radiology at the same institution in 1991. He obtained his master's (MS) degree in radiology at the Tongji Medical University in China in1994 after he received training in combined neuroradiology and abdominal imaging and after he completed his MS thesis on "Intravenous bolus dynamic enhanced permeability CT: a novel technique for intracranial tumors and vascular anatomy and diseases" mentored by Prof. Dr. Chengyuan Wang. He subsequently became a radiologist at the Chinese University Teaching Hospitals in Shenzhen, China.

In 1999, he worked at the Erasmus MC, University Medical Center Rotterdam, the Netherlands, where he discussed with Prof. Dr. Gabriel P. Krestin, Chairman of the Department of Radiology, the opportunity to do his Ph.D. and completed his radiology clinical research program in 2001. Subsequently, mentored by Prof. Dr. Hedvig Hricak, he did a research fellowship on MRI of the prostate at Memorial Sloan-Kettering Cancer Center in New York, USA, the results of which can be found in this book.

The board of Directors and the Program Committee of the Radiological Society of North America (RSNA) presented the 2004 Research Trainee Award to him for one of his RSNA presentations, which was part of his Ph.D. research. Subsequently, a Reuters Health article discussed the publication of his work showing that MRI improved prostate cancer staging nomograms. He has served as an invited reviewer for scientific journal: European Urology, and was invited to referee papers in his area of expertise for Nature Clinical Practice Urology. He is a member of several international societies (RSNA, ARRS, ASCO, SUR, AUR, NYAS) and is the author of over 20 original articles. 
16 


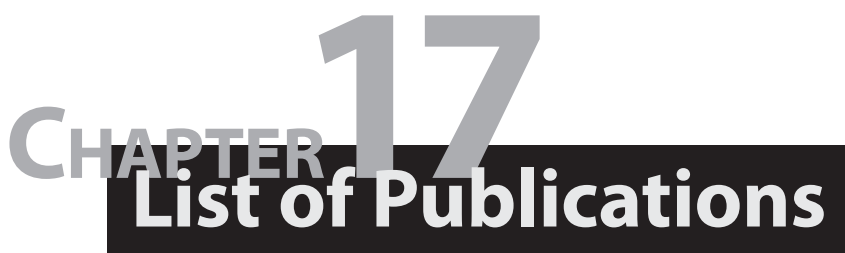




\section{List of Publications}

\section{Journal Papers}

1. L Wang, Y Mazaheri, J Zhang, NM Ishill, K Kuroiwa, H Hricak. Assessment of biological aggressiveness of prostate cancer: Correlation of MR signal intensity with Gleason grade on whole-mount step-section pathologic analysis after radical prostatectomy. (accepted for publication in Radiology)

2. JF Donohue, L Wang, H Hricak, HN Chen, JA Eastham, PT Scardino, MK Kattan.The role of MRI in improving pre-operative nomograms to predict biochemical recurrence-free probability in patients undergoing radical prostatectomy. Journal of Urology (submitted)

3. L Wang, IC vd Bos, SM Hussain, PMT Pattynama, MW Vogel, GP Krestin. Postprocessing of dynamic gadoliniumenhanced MRI of abdominal tumors (prostate cancer as one of paradigms): explanation and potential clinical application of color-coded qualitative and quantitative analysis. (accepted for publication in Acta Radiologica )

4. J Zhang, RA Lefkowitz, L Wang, NM Ishill, CS Moskowitz, P Russo, H Hricak. Significance of peritumoral vascularity on CT in evaluation of renal cortical tumor. Journal of Computer Assisted Tomography (in press, 2007)

5. L Wang, H Hricak, MW Kattan,HN Chen, K Kuroiwa, HF Eisenberg, PT Scardino. Prediction of seminal vesicle invasion in prostate cancer: Incremental value of adding endorectal MR imaging to the Kattan nomogram. Radiology 242:182-8 (2007)

6. L Wang, J Zhang, LH Schwartz, H Eisenberg, NM Ishill, CS Moskowitz, P Scardino, H Hricak. Incremental value of multiplanar cross-referencing for prostate cancer staging with endorectal MRI. American Journal of Roentgenology 188:99-104 (2007)

7. B Zhao, LH Schwartz, L Jiang, J Colville, CS Moskowitz, L Wang, R Leftowitz, F Liu, J Kalaigian. Shape-constraint region growing for delineation of hepatic metastases on contrast-enhanced computed tomograph scans. Investigative Radiology 41:753-62 (2006)

8. J Yan, B Zhao, L Wang, A Zelenetz, LH Schwartz. Marker-controlled watershed for lymphoma segmentation in sequential CT images. Medical Physics 33:2452-60 (2006)

9. SR Grobmyer, L Wang, M Gonen, Y Fong, D Klimstra, M D'angelica, RP Dematteo, LH Schwartz, LH Blumgart, WR Jarnagin. Perihepatic lymph node assessment in patients undergoing partial hepatectomy for malignancy. Annals of Surgery 244:260-4 (2006)

10. LH Schwartz, JA Colville, MS Ginsberg, L Wang, M Mazumdar, J Kalaigian, H Hricak, D Ilson, GK Schwartz. Measuring tumor response and shape change on CT: Esophageal cancer as a paradigm. Annals of Oncology 17:1018$23(2006)$

11. L Wang, H Hricak, MK Kattan, HN Chen, L Schwartz, S Eberhardt, PT Scardino. Combined endorectal and phased array MRI in the prediction of pelvic lymph node metastasis in prostate cancer. American Journal of Roentgenology 186:743-8 (2006)

12. L Wang, H Hricak, MK Kattan, HN Chen, PT Scardino. Prediction of organ-confined prostate cancer: the incremental value of MR imaging and MR spectroscopic imaging to the prostate cancer staging nomograms. Radiology 238:597-603 (2006)

13. F Liu, B Zhao, PK Kijewski, L Wang, LH Schwartz. Liver segmentation for CT images using GVF snake. Medical Physics 32: 3699-706 (2005) 
14. B Zhao, LH Schwartz, CS Moskowitz, L Wang, MS Ginsberg, CA Cooper, L Jiang, JP Kalaigian. Pulmonary metastases: effect of CT section thickness on measurement--initial experience. Radiology 234:934-9 (2005)

15. L Wang, M Mullerad, HN Chen, S Eberhardt, MK Kattan, PT Scardino, H Hricak. Prediction of prostate cancer extracapsular extension: the incremental value of endorectal coil magnetic resonance imaging. Radiology 232:133-9 (2004)

16. M Mullerad, H Hricak, L Wang, HN Chen, MK Kattan, P Scardino. Detection of prostate cancer extracapsular extension on magnetic resonance imaging: genitourinary MRI and general body MRI radiologists. Radiology 232:140-6 (2004)

17. H Hricak, L Wang, DC Wei, FV Coakley, O Akin, VE Reuter, M Gonen, MW Kattan, CN Onyebuchi, PT Scardino. The role of preoperative endorectal magnetic resonance imaging in the decision regarding whether to preserve or resect neurovascular bundles during radical retropubic prostatectomy. Cancer 100:2655-63 (2004)

18. LHSchwartz, M Mazumdar, L Wang, A Smith, S Marion, DM Panicek, RJ Motzer. Response assessment classification in patients with advanced renal cell carcinoma treated on clinical trials. Cancer 98:1611-9 (2003)

19. L Wang, YH Li, KM Wang, et al. [Radiograph films processing and quality evaluation in teleradiology]. Chinese Journal of Radiology (Chinese) 33:33-6 (1999)

20. L Wang, LJ Cai. [Intravenous bolus dynamic enhanced permeability CT: A novel technique for vascular anatomy and diseases]. Intercommunion Medicine (Chinese) 10:1-3 (1996)

21. L Wang, CY Wang, M Xiao, et al. Intravenous bolus dynamic enhanced permeability CT: A novel technique for intracranial tumors. Periodical of Applied Radiology (Chinese) 12:11-6 (1996)

22. L Wang, CY Wang, RP Chen. [CT diagnostic features of Choroid plexus papillary carcinoma]. Chinese Journal of Radiology (Chinese) 29:558-60 (1995)

23. L Wang, CY Wang, DY Feng, et al. [CT study on distribution of contrast media by single/double phase intravenous bolus injection]. Journal of Clinical Radiology (Chinese) 14:15-9 (1995)

24. L Wang, CY Wang, JF Wang, et al. [The adverse reactions of contrast media with different flow rates and single/ double phase intravenous bolus injection in dynamic enhanced permeability CT]. Journal of Clinical Radiology (Chinese) 13:1-5 (1994)

25. L Wang, CY Wang, [Dynamic enhanced permeability CT: a novel technique]. Abstracts of Foreign Medical Radiology (Chinese) 13:152-4 (1994)

\section{Conference Papers}

1. F Liu, B Zhao, P Kijewski, MS Ginsberg, L Wang, LH Schwartz. Automatic liver contour segmentation using GVF snake. Medical Imaging 2004: Image Processing, J. Michael Fitzpatrick and Milan Sonka ( eds.), vol. 5370 of Proceedings of SPIE, SPIE Press, San Diego, CA, February 2004, pp. 1466-1473

2. B Zhao, LH Schwartz, RA Lefkowitz, L Wang. Measuring Tumor Burden: Comparison of automatic and manual techniques. Medical Imaging 2004: Image Processing, J. Michael Fitzpatrick and Milan Sonka ( eds.), vol. 5370 of Proceedings of SPIE, SPIE Press, San Diego, CA, February 2004, pp. 1695-1700 


\section{Conference Abstracts}

1. L Wang, LH. Schwartz, M Mazumdar, A. Smith, NE. Kemeny. Robustness of response: A new measure of response assessment: its impact on metricies of response in colorectal cancer. ASCO 2005: 41st Annual meeting, American Society of Clinical Oncology, Orlando, FL, USA, May 13 - 17, 2005. in Journal of Clinical Oncology, vol. 23 (16), June, 2005, p263

2. L Wang, H Hricak, MK Kattan, HN Chen, PT Scardino. Prediction of prostate cancer organ confined disease: The incremental value of endorectal coil magnetic resonance imaging to Partin staging nomograms (2001 version). RSNA 2004: 90th Scientific Assembly and Annual Meeting of the Radiological Society of North America, Chicago, IL, USA, November 28 - December 3, 2004. in Radiology, vol. 233(P), November, 2004, p270

3. B Zhao, L H Schwartz, L Jiang, J A Colville, L Wang, R A Lefkowitz, et al. Automatic measurements of hepatic metastases on contrast enhanced CT scan - comparison with radiologists' manual results. RSNA 2004: 90th Scientific Assembly and Annual Meeting of the Radiological Society of North America, Chicago, IL, USA, November 28 - December 3, 2004. in Radiology, vol. 233(P), November, 2004, p277

4. JA Colville, MJ Gollub, L Wang, P Paty, LH Schwartz. Recurrent rectal carcinoma: MRI evaluation. RSNA 2004: 90th Scientific Assembly and Annual Meeting of the Radiological Society of North America, Chicago, IL, USA, November 28 - December 3, 2004. in Radiology, vol. 233(P), November, 2004, p436

5. L Wang, G.K. Abou-Alfa, F. Lui, L.B. Saltz, J. Kalagian, B. Zhao, J. Colville, J. Nyoro, B. Schwartz, L. Schwartz. Novel methodology of response assessment in hepatocellular carcinoma (HCC) - Assessing response by change in tumor enhancement in distinction to conventional means. ASCO 2004: 40th Annual meeting, American Society of Clinical Oncology, New Orleans, Louisiana, USA, June 5 - 8, 2004. in Journal of Clinical Oncology, vol. 22 (14), Jul, 2004, p221

6. L.H. Schwartz, J.A.Colville, L.Wang, H.Hricak, D.llson, G.Schwartz. Reponse assessment parameters in esophageal cancer- conventional and novel techniques. ASCO 2004: 40th Annual meeting, American Society of Clinical Oncology, New Orleans, Louisiana, USA, June 5 - 8, 2004. in Journal of Clinical Oncology, vol. 22 (14), Jul, 2004, p321

7. SR Grobmyer, L Wang, Y Fong, M D'Angelica, M Gonen, LH. Schwartz, LH. Blumgart, WR. Jarnagin. A prospective analysis of perihepatic lymph node status in patients undergoing hepatic resection for malignancy. IHPBA 2004: 6th World Congress of the International Hepato-Pancreato-Biliary Association, Washington, DC, USA, June 2 - 6, 2004. in Proceedings of 6th World Congress of the International HepatoPancreato-Biliary Association, June 2004, p120

8. L Wang, H Hricak, S Eberhardt, HN Chen, M Kattan, P Scardino. Prostate cancer - value of 3D Endorectal MR Imaging in the evaluation of seminal vesicle invasion in patients treated by radical prostatectomy. ARRS 2004: 104th annual meeting of the American Roentgen Ray Society, Miami, FL, USA, May 2-7, 2004. in AJR Am J Roentgenol, vol. 182 (4), May 2004, p67

9. L Wang, H Hricak, S Eberhardt, HN Chen, M Kattan, P Scardino. Prostate Cancer: Combined use of eMRI and Partin Nomogram 2001 in the Prediction of pelvic lymph node metastasis. ARRS 2004: 104th annual meeting of the American Roentgen Ray Society, Miami, FL, USA, May 2-7, 2004. in AJR Am J Roentgenol, vol. 182 (4), May 2004, p117

10. LH Schwartz, B Zhao, L Jiang, M Mazumdar, L Wang, NE Kemeny. Automated hepatic metastasis tumor assessment on imaging. RSNA 2003: 89th Scientific Assembly and Annual Meeting of the Radiological Society of North America, Chicago, IL, USA, November 30 - December 5, 2003. in Radiology, vol. 229(P), November, 2003, p363

11. B Zhao, LH Schwartz, CS Moskowitz, MS Ginsberg, L Wang, L Jiang, C Cooper, J Kalagian. Automated tumor measurement: impact of CT slice thickness on measurement techniques and results. RSNA 2003: 89th 
Scientific Assembly and Annual Meeting of the Radiological Society of North America, Chicago, IL, USA, November 30 - December 5, 2003. in Radiology, vol. 229(P), November, 2003, p556

12. B Zhao, L Wang, J Kalaigian, P Kijewski, LH Schwartz. Quantification of body fat distribution in the abdomen and pelvis on CT with automated fat segmentation. RSNA 2003: 89th Scientific Assembly and Annual Meeting of the Radiological Society of North America, Chicago, IL, USA, November 30 - December 5, 2003. in Radiology, vol. 229(P), November, 2003, p573

13. L Wang, L Schwartz, M Mazumdar, A Smith, NE Kemeny, D Panicek. Impact of measurement technique on response assessment criteria in colorectal cancer. ASCO 2003: 39th Annual meeting, American Society of Clinical Oncology, Chicago, IL, USA, May 3 - June 3, 2003. in Journal of Clinical Oncology, vol. 22 , June, 2003, p278

14. L.H. Schwartz, M.Mazumdar, L.Wang, A.Smith, S. Marion, D. Panicek, R. Motzer. Effect of response criteria on outcome assessment for patients with advance renal cell carcinoma. ASCO 2003: 39th Annual meeting, American Society of Clinical Oncology, Chicago, IL, USA, May 3 - June 3, 2003. in Journal of Clinical Oncology, vol. 22, June, 2003, p385

15. L Wang, H Hricak, S Eberhardt, J Qin, MK Kattan, P Scardino. Prostate cancer: predicting extracapsule invasion: incremental value of endorectal MRI to finding of perineural invasion on sextant biopsy. RSNA 2002: 88th Scientific Assembly and Annual Meeting of the Radiological Society of North America, Chicago, IL, USA, December 1-6, 2002, in Radiology, vol. 225(P), November, 2002, p123

16. L Wang, Hricak H, Qin J, Koutcher J, Kattan M, Scardino P. Prostate cancer: combined use of eMRI and updated Partin nomogram in the prediction of extracapsular extension. RSNA 2002: 88th Scientific Assembly and Annual Meeting of the Radiological Society of North America, Chicago, IL, USA, December 1-6, 2002, in Radiology, vol. 225(P), November, 2002, p629

17. L Wang, D Buljevac, EH Oei, ZH Flach. Relapsing-remitting multiple sclerosis: influence of clinical infection on T2 lesion load on magnetic resonance imaging. RSNA 2001: 87th Scientific Assembly and Annual Meeting of the Radiological Society of North America, Chicago, IL, USA, November 25-30, 2001, in Radiology, vol. 221(P), November, 2001, p134

18. BV Heerebeek, SM Hussain, MC Kock, L Wang, MG Hunink. MRI: a one-stop shop imaging modality for the preoperative evaluation of potential living renal donors. RSNA 2001: 87th Scientific Assembly and Annual Meeting of the Radiological Society of North America, Chicago, IL, USA, November 25-30, 2001, in Radiology, vol. 221(P), November, 2001, p709

19. L Wang, MC Kock, HA Vrooman, SH Hussain, GP Krestin GP, MG Hunink.What are really interactive 3-

dimensional MRI reconstructions in evaluation of the living renal donor? RSNA 2001: 87th Scientific Assembly and Annual Meeting of the Radiological Society of North America, Chicago, IL, USA, November 25-30, 2001, in Radiology, vol. 221(P), November, 2001, p728

20. L Wang, SM Hussain, BV Heerebeek, PM Pattynama, MW Vogel, GP Krestin. Postprocessing of dynamic gadolinium-enhanced MRI exams of the upper abdominal organs: explanation and potential clinical application of color-coded qualitative and quantitative analysis. RSNA 2000: 86th Scientific Assembly and Annual Meeting of the Radiological Society of North America, Chicago, IL, USA, November 25-30, 2000, in Radiology, vol. 217 (P), November, 2000, p635

21. L Wang. Dynamic computed tomography in the characterization of hepatocellular carcinoma and cavernous hemangioma. The third Chinese Medical Radiology Conference. Xi-An, April 1997.

22. L Wang, CY Wang,M Xiao, etal. Intravenous bolus dynamic enhanced permeability CT: a novel technique for vascular anatomy and diseases. The fourth Chinese Cerebrovascular Diseases Conference. Cheng-Du, October 12, 1995 
23. L Wang. Dynamic contrast-enhanced permeability CT and surgical correlations in the evaluation of intracranial tumors and intracranial vascular pathologies. the seventh Chinese Congress of Radiology (CCR), the Chinese Society of Radiology (CSR) of the Chinese Medical Society (CMA); the conference of the Chinese Society of Radiologic Technologists (CSRT) of the Chinese Medical Society (CMA); the Chinese Convention of Excellent Medical Radiologic Comparative Film Appraisal. In Honor of the Centennial Anniversary of the Discovery of X-rays by Wilhelm Conrad Röntgen. Beijing, China, June 1995

24. L Wang, Wang CY. Dynamic enhanced permeability brain CT. The National Neuroradiology Seminar, Suzhou, China, 1992 
17 


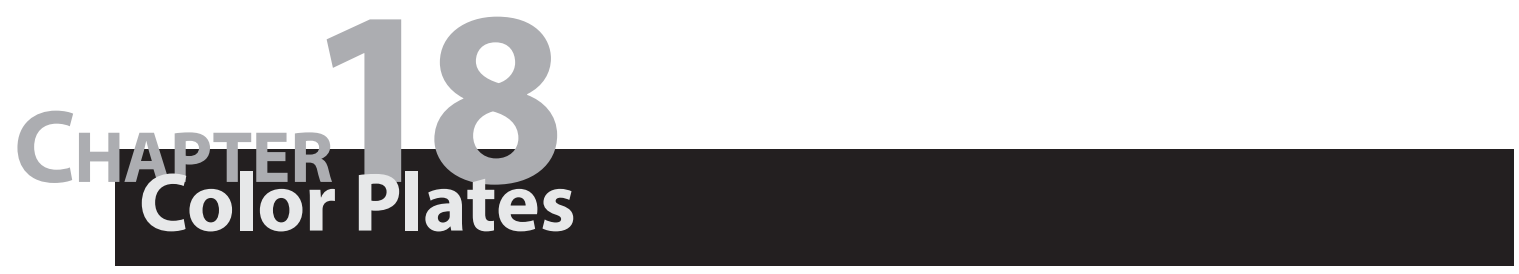




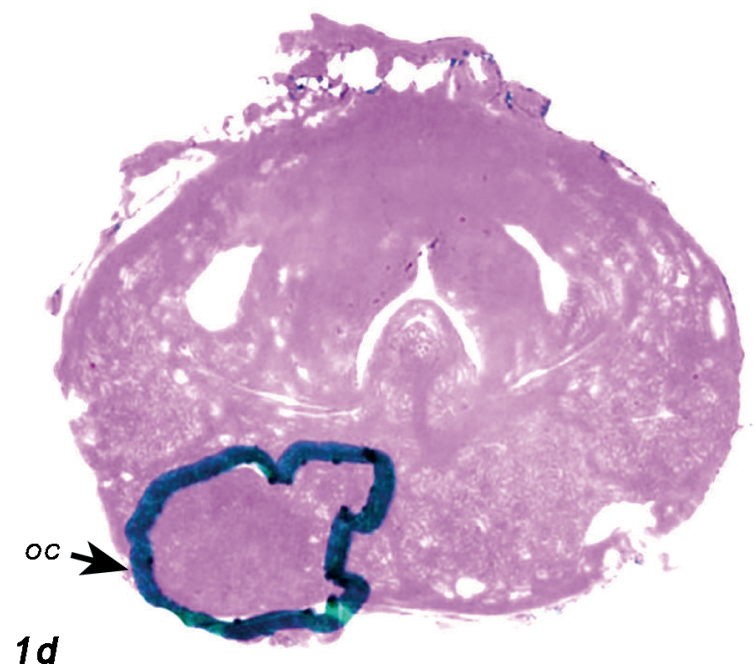

Figure 1d: Images in a 55-year-old man with a small palpable clinical stage T2 prostate nodule and PSA level of $0.80 \mathrm{ng} / \mathrm{mL}$. Sextant biopsy results showed Gleason grade $3+3$ cancer involving 10\% of submitted tissue from the right side (50\% of the cores) and no perineural invasion. By using staging nomograms, the likelihood of OCPC was determined to be $81 \%$. (a-c) T2-weighted fast spinecho MR images. (a) Transverse 3-mm-thick MR (4900/118) image shows OCPC (arrow) in the right apex. (b) Sagittal 4-mm-thick MR (6000/97) image shows a focus of cancer (arrow) in the right apex and midgland. (c) Coronal 3-mm-thick MR (4666/96) image shows cancer (arrow) in the right apex and midgland. (d) Whole-mount serial section of the removed prostate shows organ-confined cancer (arrow) involving the right posterior quadrant of the prostate.

\section{Chapter 3}

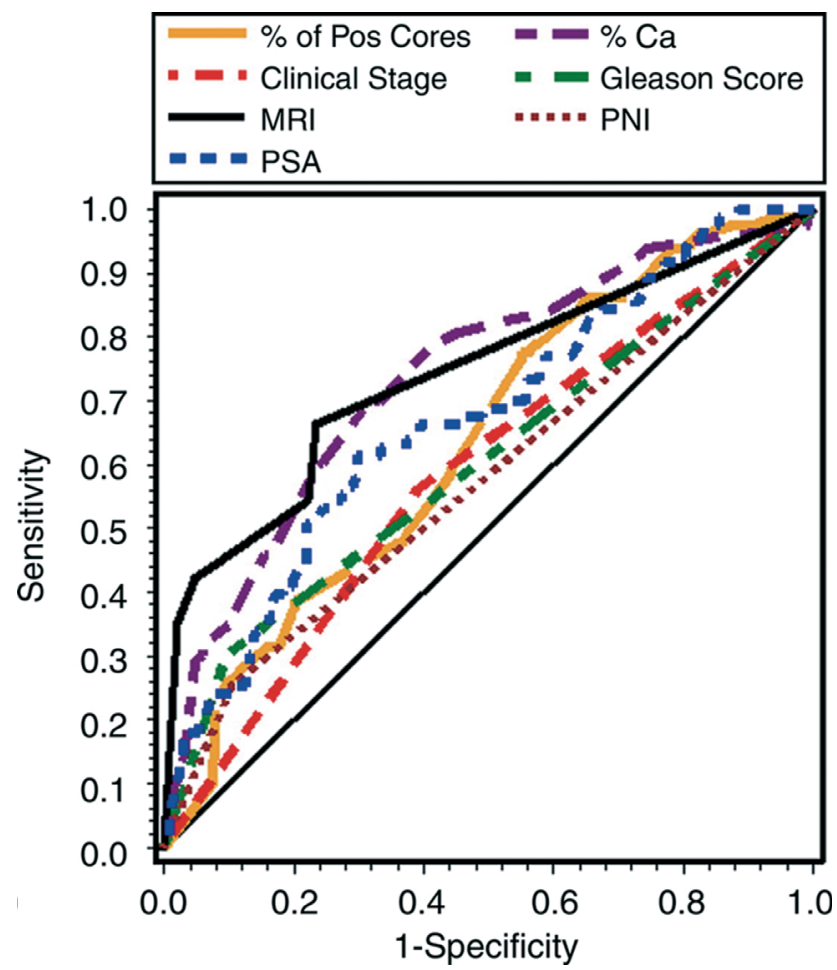

Figure 1. Graph shows comparison of ROC curves of seven variables for prediction of ECE. Area under ROC curve for MR imaging findings is 0.743 . Diagonal line indicates area under ROC curve of 0.500 (ie, no separation between patients with and patients without ECE). Predictor variables treated as continuous variables except for Gleason score (categorized as $3+2,3+3$, and $3+4$ vs $3+5,4+3,4+4,4+5$, and $5+5)$ and clinical stage of cancer (categorized as T1c vs T2a, T2b, and $\mathrm{T} 2 \mathrm{c}$ ). \% Ca $=$ greatest percentage of cancer in all core biopsy specimens, \% of Pos Cores = percentage of cancerpositive core specimens in all core biopsy specimens. 


\section{Chapter 4}

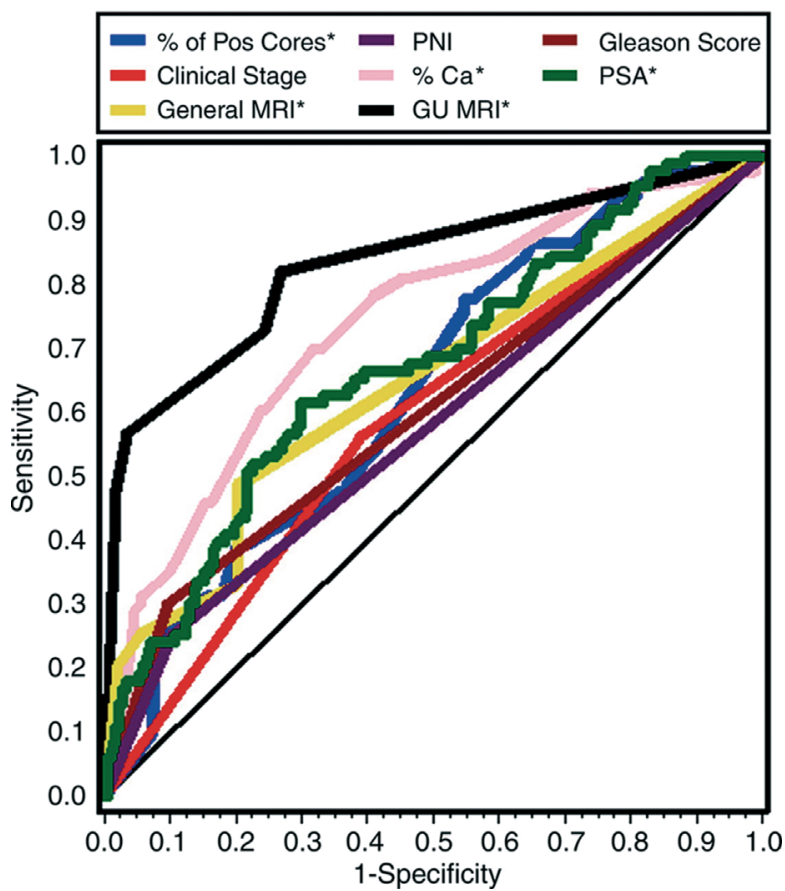

Figure 1. Graph shows ROC curves of univariate analysis results for all predictors of ECE. Predictors analyzed as continuous variables are indicated $\left(^{*}\right)$. Gleason score was categorized as $3+2$ and $3+3$ versus $3+4,4+3,3+5,4+$ $4,4+5$, and $5+5$. Clinical stage of tumor was categorized according to TNM classifications as T1c versus $\mathrm{T} 2 \mathrm{a}, \mathrm{T} 2 \mathrm{~b}$, and T2c. Diagonal line indicates area under ROC curve of 0.500 . General MRI = endorectal MR imaging findings for general body radiologist group, \% $\mathrm{Ca}$ = greatest percentage of cancer in all core biopsy specimens, GU MRI = endorectal MR imaging findings for genitourinary radiologist group, \% of Pos Cores $=$ percentage of cancer-positive core specimens in all core biopsy specimens.

\section{Chapter 5}

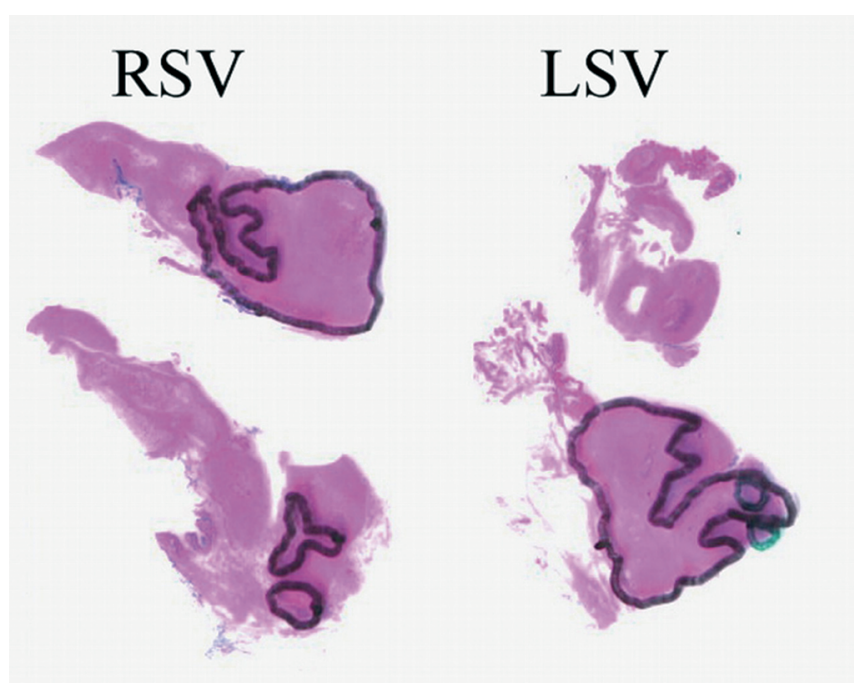

Figure 1c: pT4 prostate cancer (clinical stage T2b with PSA level of $12.42 \mathrm{ng} / \mathrm{mL}$ ) and bilateral seminal vesicle involvement in a 63-year-old man. Sextant biopsy results showed Gleason grade $4+3$ tumors involving $50 \%$ of the total submitted tissue on both sides ( $94 \%$ of total submitted cores were positive) and PNI in left peripheral and transition zones. According to the Kattan staging nomogram, the likelihood of SVI was $18 \%$. (a) Transverse T2-weighted fast spin-echo 3-mm-thick MR image (4900/118 [repetition time msec/effective echo time msec]) and (b) coronal T2-weighted fast spin-echo 3-mm-thick MR image (4666/96) show bilateral SVI (arrows). (c) Whole-mount pathologic step section shows prostate cancer with bilateral seminal vesicle involvement. The green circle represents the area with a Gleason grade of 3, and the black circle represents the area with a Gleason grade of 4 or a mixture of Gleason grades 4 and 5. LSV = left seminal vesicle, RSV = right seminal vesicle. 


\section{Chapter 6}
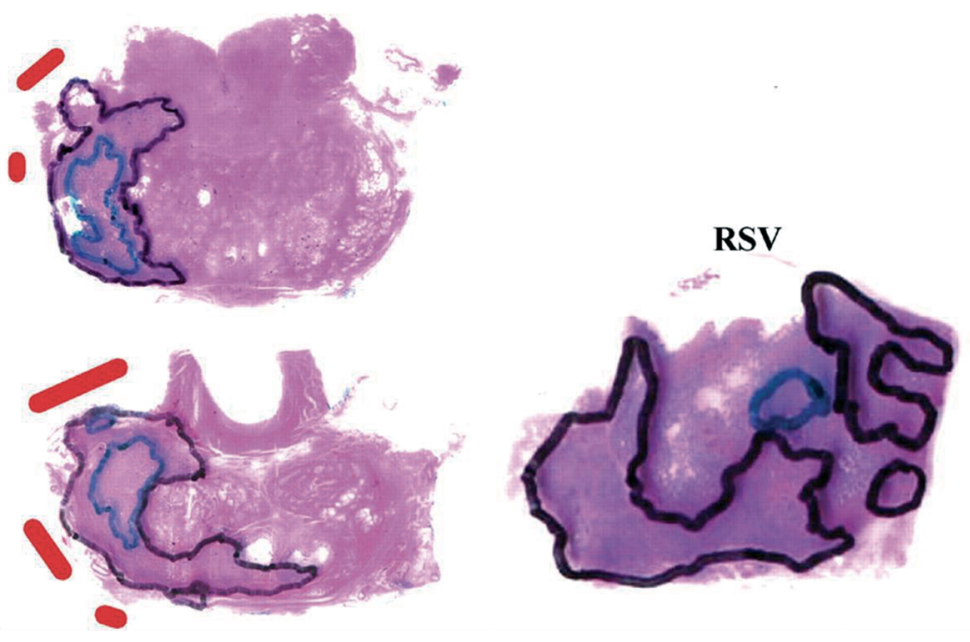

Fig. 1D: 53-year-old man with clinical stage T1c prostate carcinoma with Gleason score of $3+3$ and prostate-specific antigen level of $11.9 \mathrm{ng} / \mathrm{mL}$. MRI without PACS cross-referencing indicated possible extracapsular extension (ECE) at right base and possible right seminal vesicle invasion (SVI) (scores of 3 and 3, respectively). However, MRI with PACS cross-referencing indicated definite ECE and definite SVI (scores of 5 and 5, respectively). Histologic photograph shows whole-mount sections that confirm presence of ECE (red) at right base and right seminal vesicle invasion (blue). RSV = right seminal vesicle.

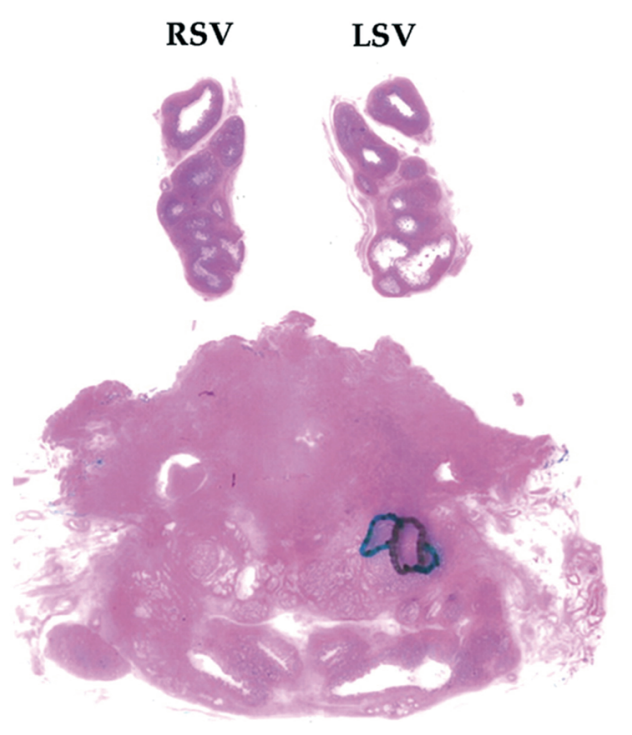

Fig. 2D: 63-year-old man with clinical stage $T 1 \mathrm{c}$ prostate carcinoma with Gleason score of $4+4$, prostate-specific antigen level of $12.9 \mathrm{ng} / \mathrm{mL}$. MRI without PACS cross-referencing indicated possible extracapsular extension (ECE) at left base and possible left seminal vesicle invasion (SVI) (scores of 3 and 3 , respectively). However, cross-referenced MR images (A-C) indicated no ECE and no SVI (scores of 1 and 1, respectively). Histopathologic photograph shows whole-mount sections confirming absence of extracapsular extension and seminal vesicle invasion at left base. RSV = right seminal vesicle, $L S V=$ left seminal vesicle. 

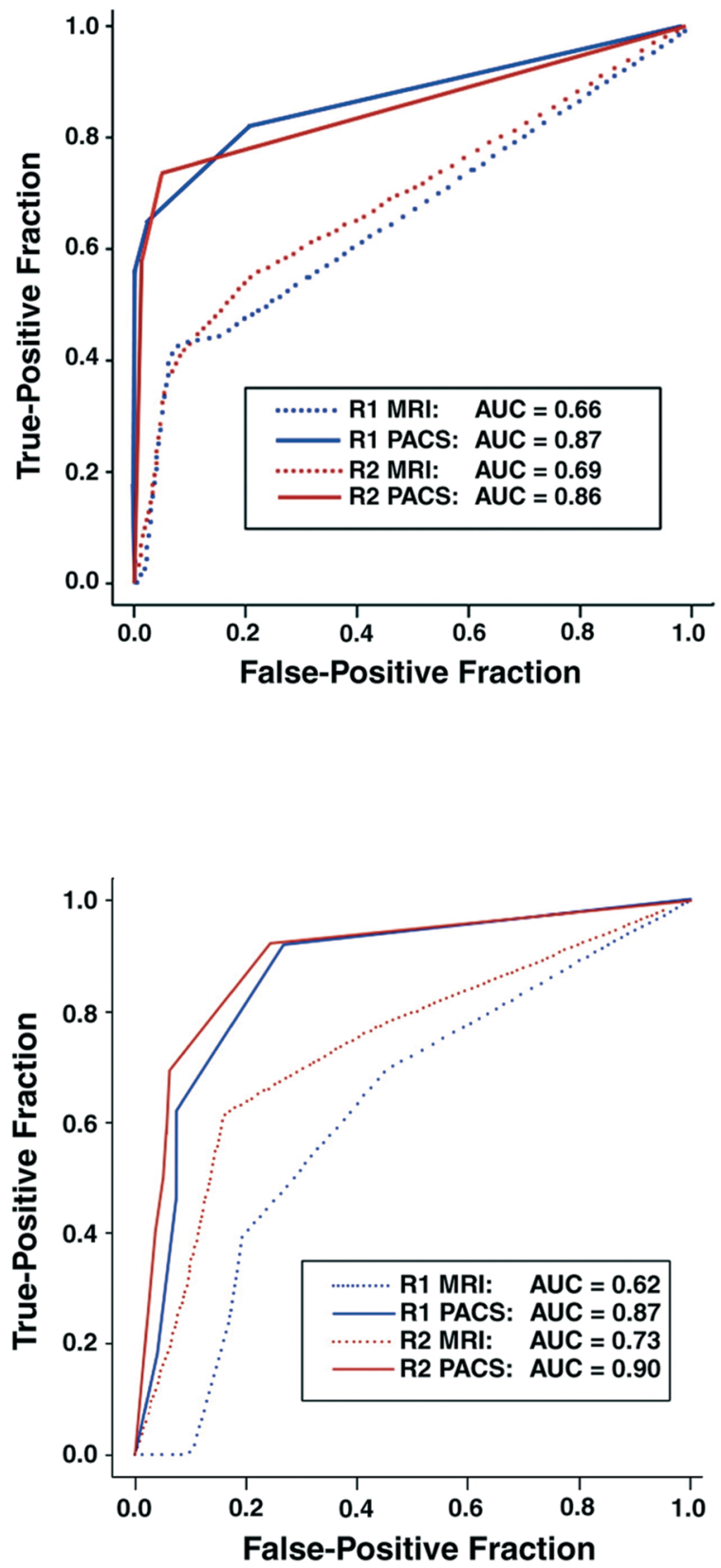

Fig. 3: Graph shows results of receiver operating characteristic analysis for detection of extracapsular extension with MRI alone and MRI with PACS cross-referencing. R1 = reviewer 1, R2 = reviewer 2, MRI = MRI alone, PACS = MRI with PACS cross-referencing, AUC $=$ area under curve
Fig. 4: Graph shows results of receiver operating characteristic analysis for detection of seminal vesicle invasion with MRI with PACS cross-referencing and MRI alone. $R 1=$ reviewer $1 ; R 2=$ reviewer 2; MRI = MRI alone; PACS = MRI with PACS cross-referencing, AUC $=$ area under curve. 


\section{Chapter 7}

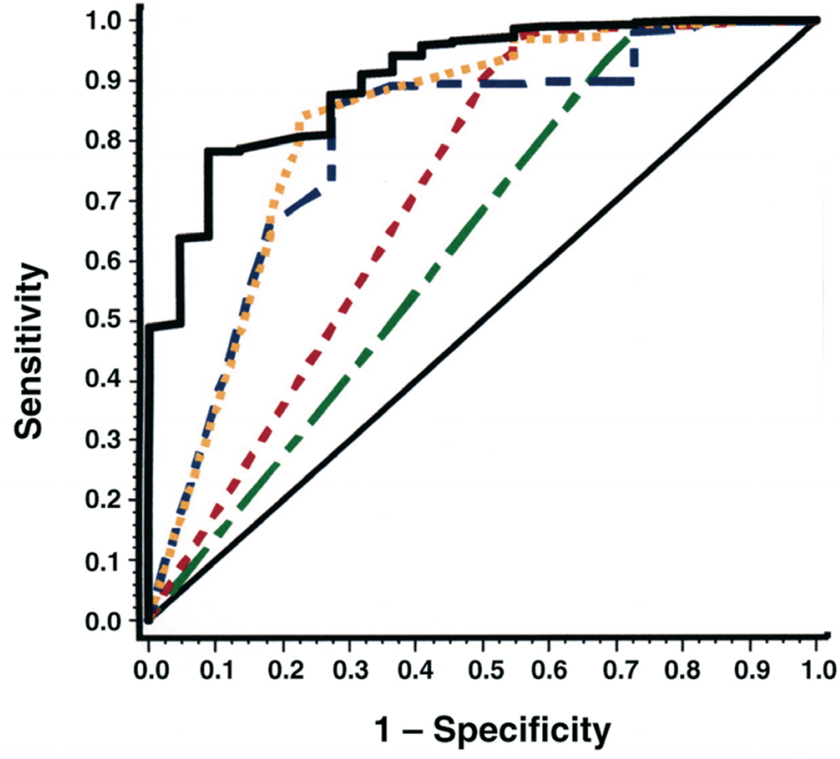

Fig. 1: Graph shows that each multivariate model had greater area under receiver operating characteristic curve (AUC) than model A (green) (Table 7), which included only MRI findings for lymph node metastasis (LMN). Model combining MRI findings for seminal vesicle invasion and LNM (model F) (red) had greater AUC than model A: 0.714 vs 0.633 , respectively $(p=0.08)$. Model combining MRI findings for extracapsular extension and LNM (model G) (blue) had greater AUC than model A: 0.798 vs 0.633 , respectively ( $p=0.15$ ). Model combining MRI findings for extracapsular extension, seminal vesicle invasion, and LNM (model H) (yellow) had greater AUC than model A: 0.813 vs 0.633 , respectively $(p=0.11)$. Model combining MRI findings for extracapsular extension, seminal vesicle invasion, and LNM and Partin tomogram prediction of LNM (model I) (black) had significantly greater AUC than model A: 0.892 vs 0.633 , respectively $(p<0.01)$. 


\section{Chapter 8}

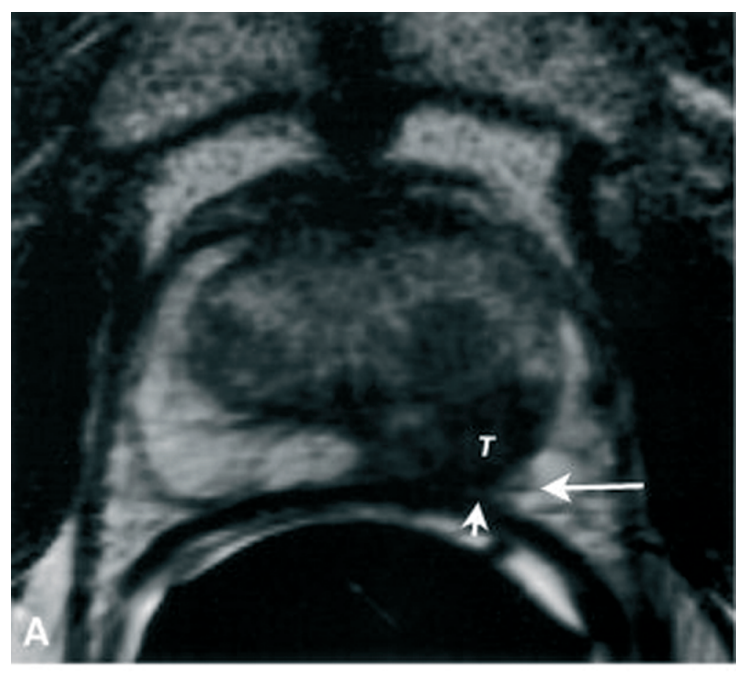

Figure 1. Case 1. Clinical stage T2a prostate carcinoma with a Gleason score of 7 in a 61-year-old patient with a prostate-specific antigen (PSA) level of $5.4 \mathrm{ng} / \mathrm{mL}$. The premagnetic resonance imaging (pre-MRI) surgical plan indicated probable preservation of the left neurovascular bundle (NVB) (a score of 2 of 5 , where 1 indicated definite preservation of the NVB and 5 indicated definite resection of the NVB) and probable resection of the right NVB (a score of 4 of 5). (A) An axial T2-weighted MRI image showed a large low-signal intensity tumor $(T)$ in the left peripheral zone; there was obliteration of the rectoprostatic angle (arrowhead) and asymmetry of the NVB (arrow); both findings indicate extracapsular extension (ECE) (MRI score of 5). No tumor was noted in the right peripheral zone. As a result, the surgical plan was changed and was more aggressive on the left NVB (changing from 2 to 5 ) and less aggressive on the right NVB (changing from 4 to 1). (B) A corresponding histopathologic step-section map demonstrated established ECE at the left posterior (base); all surgical margins were free of tumor. MRI findings appropriately changed the clinical impression of the urologist and the surgical plan for the left and right NVB.

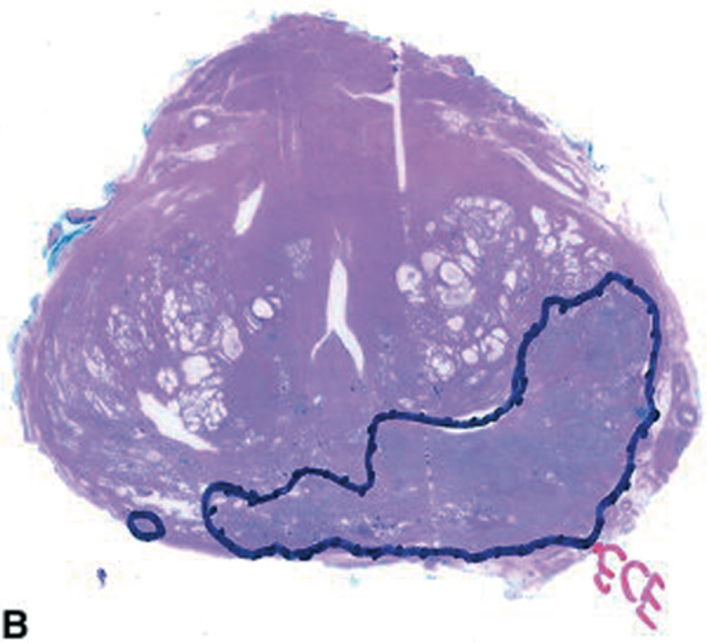



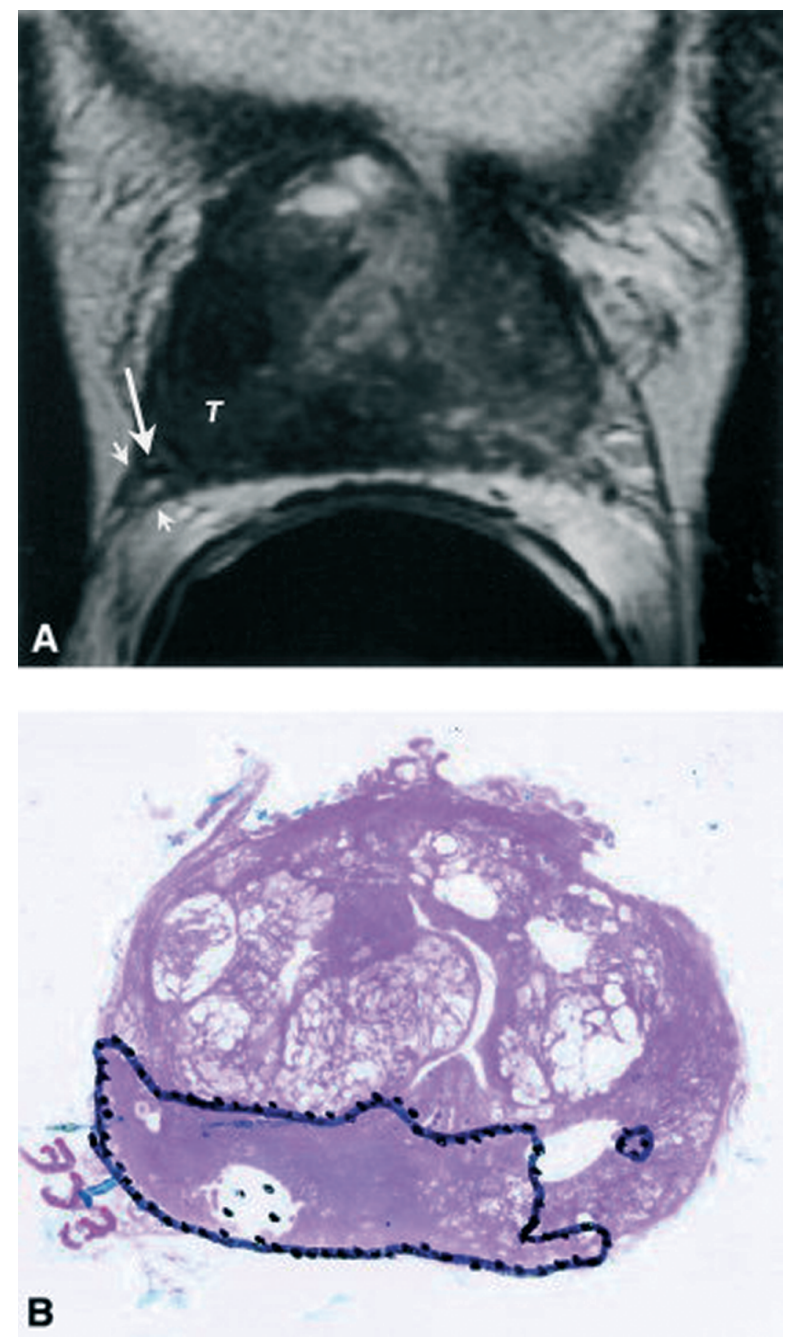

Figure 2. Case 2. Clinical stage T1c prostate carcinoma with a Gleason score of 9 in a 60-year-old patient with a prostate-specific antigen (PSA) level of $8.45 \mathrm{ng} / \mathrm{mL}$. The premagnetic resonance imaging (pre-MRI) surgical plan indicated probable preservation of the right neurovascular bundle (NVB) (a score of 2 of 5 , where 1 indicated definite preservation of the NVB and 5 indicated definite resection of the NVB) and possible resection of the left NVB (a score of 3 of 5). (A) An axial T2-weighted MRI image showed a large low-signal intensity tumor $(\mathrm{T})$ in the right peripheral zone. The contour of the gland was angulated and there was direct tumor extracapsular extension (ECE) (arrowheads), and envelopment of the right NVB (arrow); findings indicated right-sided ECE (MRI score of 5). There was no MRI evidence of left-sided ECE. As a result, the surgical plan was altered and was more aggressive with regard to the right NVB (changing from 2 to 5). The surgical plan for the left NVB remained unchanged. (B) A corresponding histopathologic step-section map showed established, posterior, right-sided ECE. In this case, aggressive management of the right NVB was appropriate. 


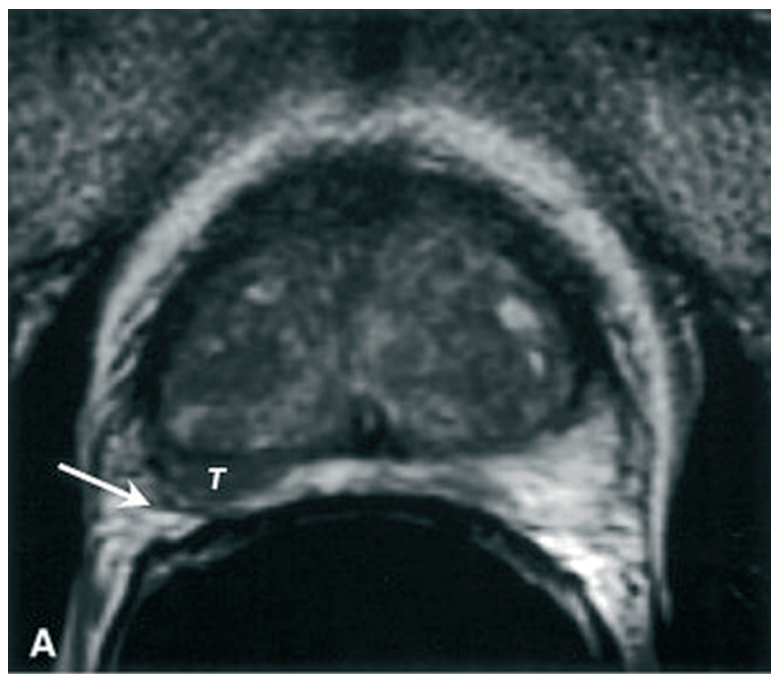

Figure 3. Case 3 . Clinical stage T1c prostate carcinoma with a Gleason score of 6 in a 55-year-old patient with a prostate-specific antigen (PSA) level of $6.31 \mathrm{ng} / \mathrm{mL}$. The promagnetic resonance imaging (pre-MRI) surgical plan indicated definite preservation of both neurovascular bundles (NVBs) (a score of 1 of 5 for both, where 1 indicated definite preservation of the NVB and 5 indicated definite resection of the NVB). (A) An axial T2-weighted MRI image demonstrated a focal low-signal intensity tumor (T) in the right peripheral zone. The tumor extended to the capsular margin in the region of the right NVB (arrow). Because the tumor abutted the right NVB, early extracapsular extension (ECE) could not be excluded (MRI score of 3). Consequently, the surgical plan was changed and was more aggressive with regard to the right NVB (changing from 1 to 3 ). MRI showed no tumor on the left. Thus, the surgical plan for the left NVB remained unchanged. (B) A corresponding histopathologic step-section map revealed no ECE and all surgical margins were free of tumor. In this case, MRI findings inappropriately changed the surgical plan of the urologist with regard to the right NVB but confirmed the decision to preserve the left NVB.

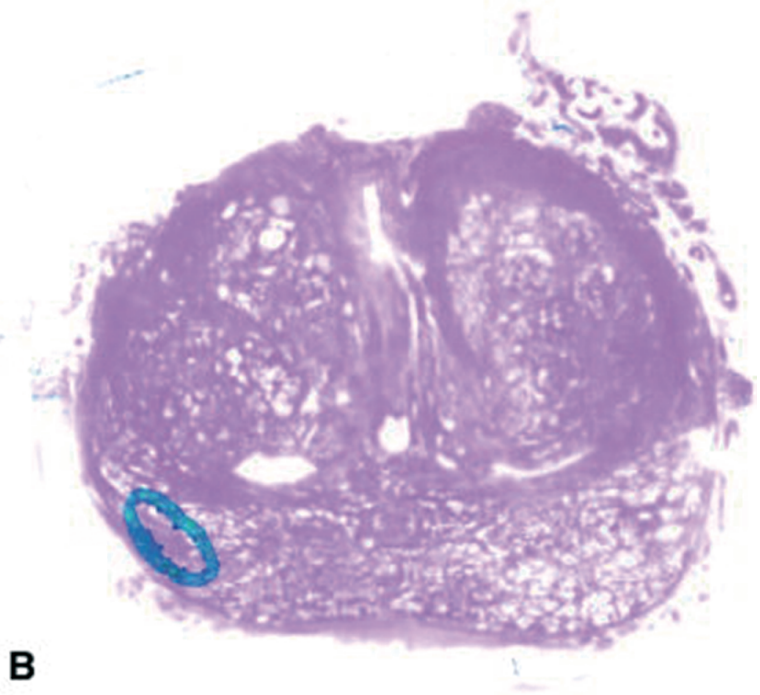




\section{Chapter 10}
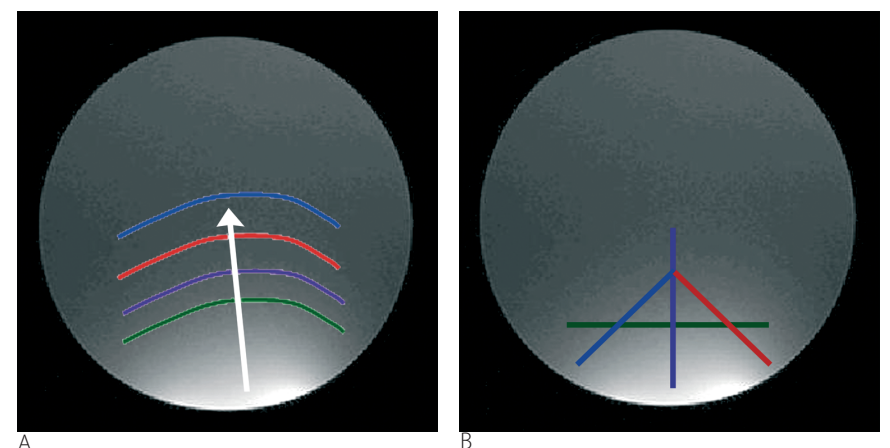

Figure 1: Generating iso-surface image profiles from the phantom study. (a) and (b) show the iso-surface and projections on a phantom, respectively; (c) and (d) show the corresponding signal intensity profiles for the configurations in (a) and (b). Signal intensity from the mid $1 / 3$ of the balloon-covered expandable endorectal coil remained homogeneous in the $z$ dimension (e).
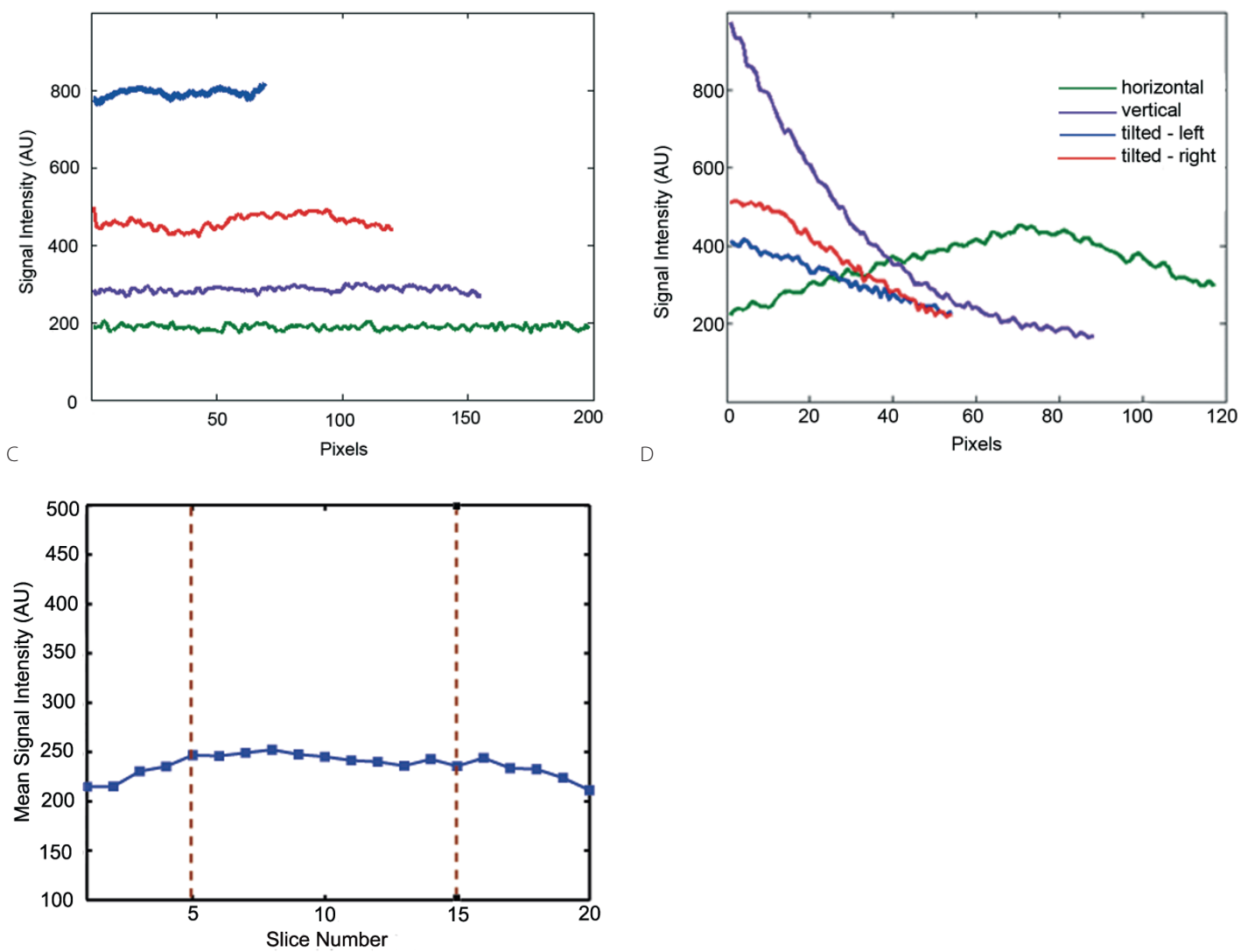

E 

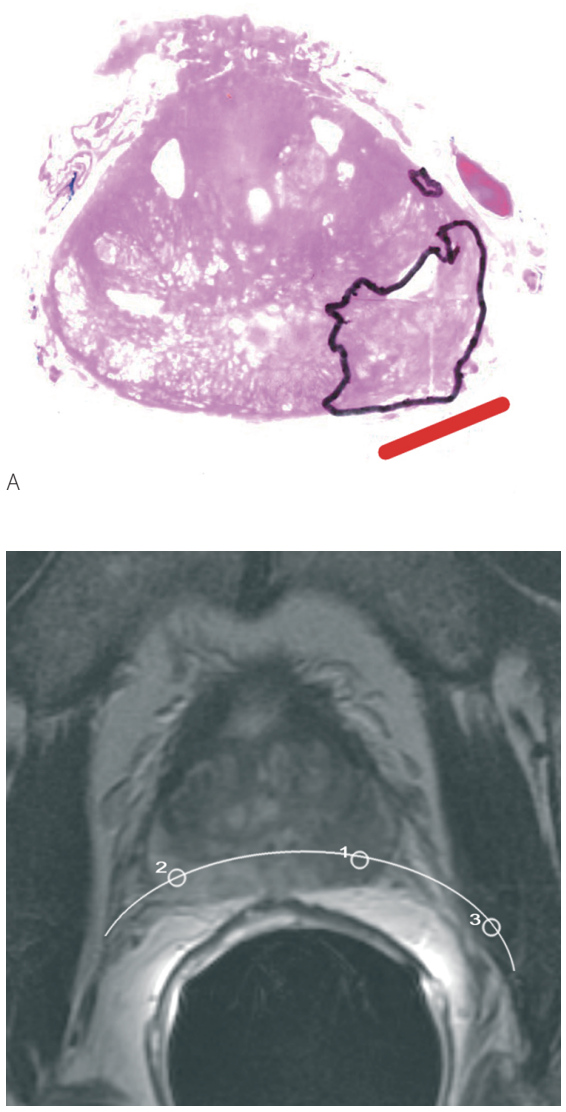

Figure 2: 54 -year-old male with Gleason grade 4 prostate cancer in the peripheral zone (PZ) (weight $100.2 \mathrm{~kg}, \mathrm{PSA} 4.4 \mathrm{ng} / \mathrm{ml}$, clinical stage T2b, pathologic stage T3b). (a) Whole-mount step-section pathologic tumor map shows an index lesion of Gleason grade 4 in left PZ. The black circle represents the area of Gleason grade 4 . (b) On the corresponding transverse uncorrected T2-weighted MR image (repetition time msec/echo time msec, 4916.7/102.48; echo train length, 12; field of view, 14 cm; section thickness, $3 \mathrm{~mm}$; no section gap) ROls were placed on the center of the tumor (ROI-1) on non-tumor prostatic tissue (ROI-2) and on internal obturator muscle (ROI-3). The PZ tumor-to-muscle SIR was 2.27. The PZ non-tumor prostatic tissue-tomuscle SIR was 4.86. (c) On the corresponding transverse T2-weighted MR image corrected with prostatic analytical coil correction (PACC) software, ROls were placed the center of the tumor using (ROI-4) on contralateral non-tumor prostatic tissue (ROI-5) and on internal obturator muscle (ROI-6). The PZ tumor-to-muscle SIR was 1.82. The $\mathrm{PZ}$ non-tumor prostatic tissue-to-muscle SIR was 3.44

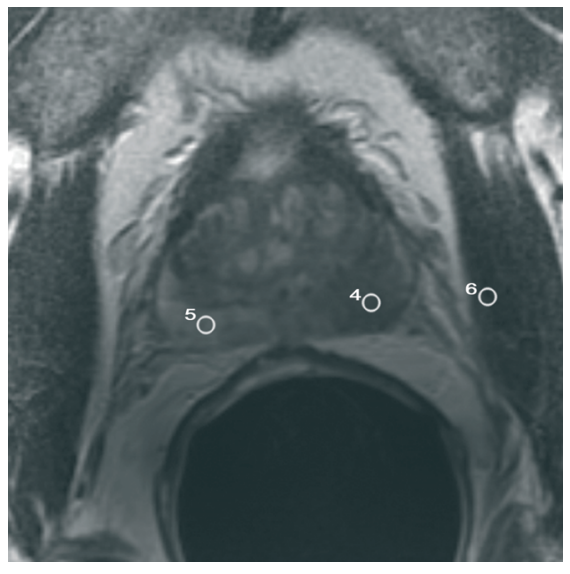

C 
CAHAPTHR

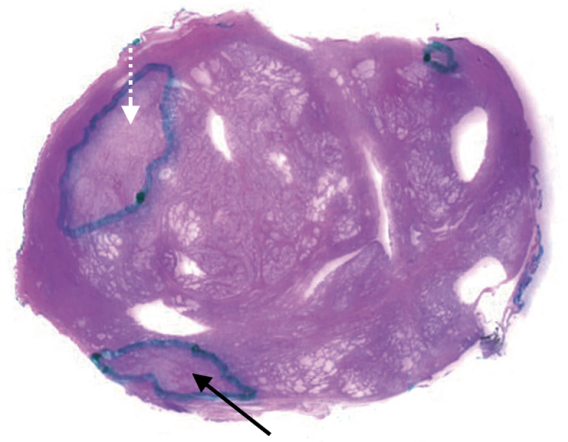

A

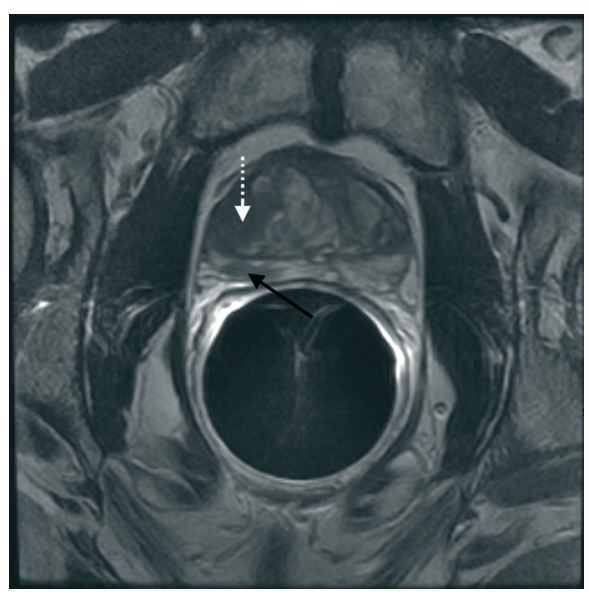

B

Figure 3: 60 -year-old male with Gleason grade 3 prostate cancers in the PZ and TZ (weight $80.7 \mathrm{~kg}$, PSA $5.1 \mathrm{ng} / \mathrm{ml}$, clinical stage T2b, pathologic stage T2c). (a) Whole-mount step-section pathologic tumor map shows an index lesion of Gleason grade 3 in the right TZ (dashed white arrow), and a second dominant lesion of Gleason grade 3 (black arrow) in the right posterior PZ. The outlined areas represent the two tumors. (b) Corresponding transverse T2-weighted MR image (TR/effective TE = 4000/103; echo-train length, 16; field of view, $14 \mathrm{~cm}$; acquisition matrix, $256 \times$ 192; section thickness, 3 mm; no section gap) corrected with PACC software shows the index Gleason grade3 lesion (dashed white arrow) in the TZ, and the second dominant Gleason grade 3 lesion (black arrow) in the PZ. The tumor-to-muscle SIR (1.77) in the TZ is lower than that in the PZ (3.99). Also note that the PZ tumor-to-muscle SIR in this Gleason grade 3 lesion (3.99) is higher than that of the Gleason grade 4 lesion (1.82) in a different patient shown in Figure 2. 


\section{Chapter 11}
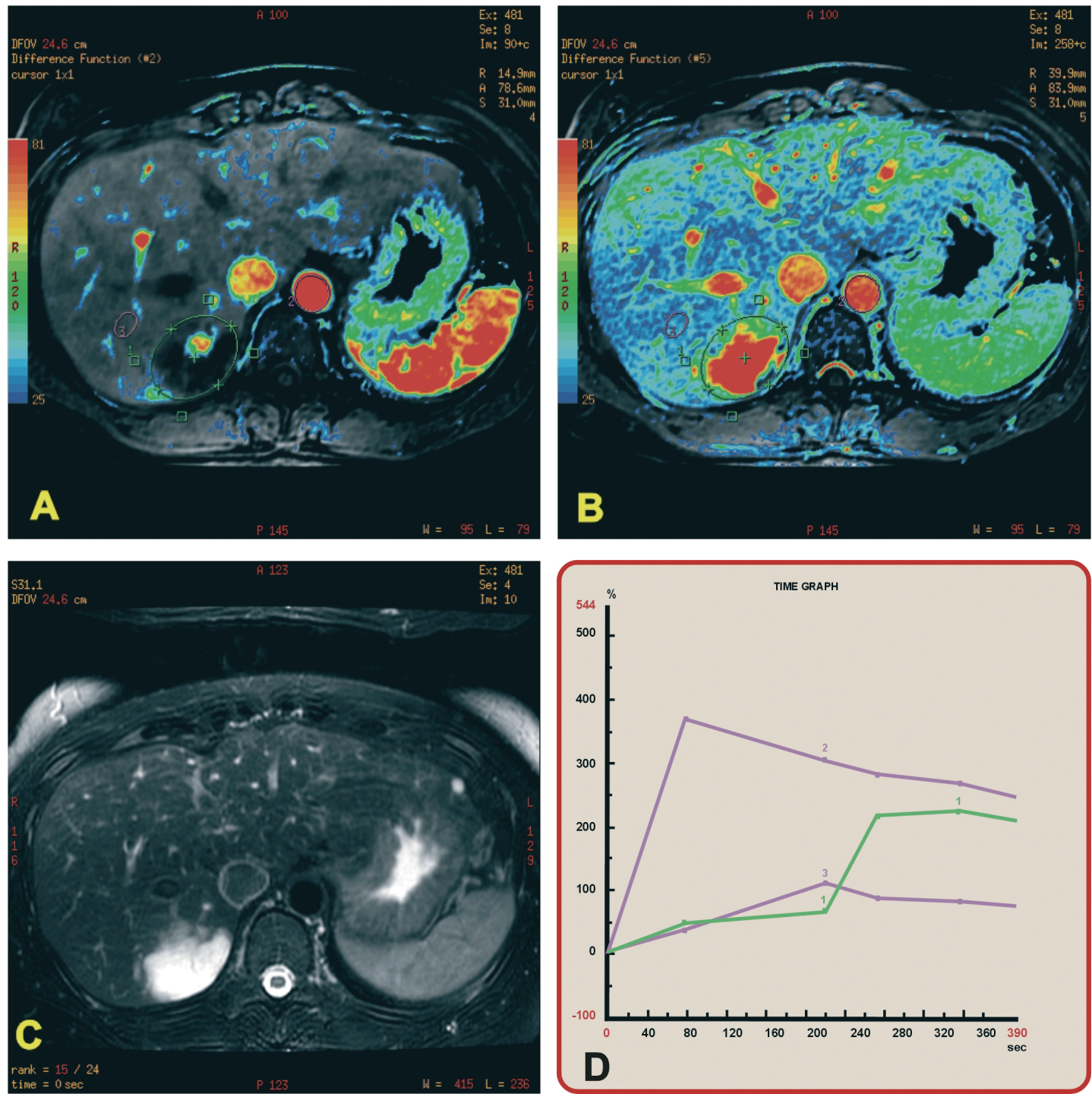

Figure 3: Hemangioma

40-year-old female with a $48 \times 35 \mathrm{~mm}$ hemangioma in the right lobe of the liver. A) (Color-coded parameter image from the arterial phase, calculated using relative enhancement) Nodular enhancement in the periphery of the lesion. B) (Color-coded parameter image from the delayed phase, calculated using relative enhancement) Complete fill-in of the entire lesion. The lesion is entirely red. C) (T2-weighted image, TR/TE=1059/99.4 msec) High signal intensity, lobulated and well-circumscribed lesion typical for hemangioma. D) The time-intensity curve from the ROI of the lesion (green curve) reflects the steady enhancement of hemangioma (atypical Type I curve). The time-intensity curve from the ROI of the blue curve (line 3) shows normal liver parenchyma enhancement, and line 2 represents the signal intensity within the aorta at the acquired vascular phase. Note: decremental value in color spectrum: red $>$ orange $>$ yellow $>$ green $>$ blue $>$ indigo $>$ violet. 
CAHAPTHR
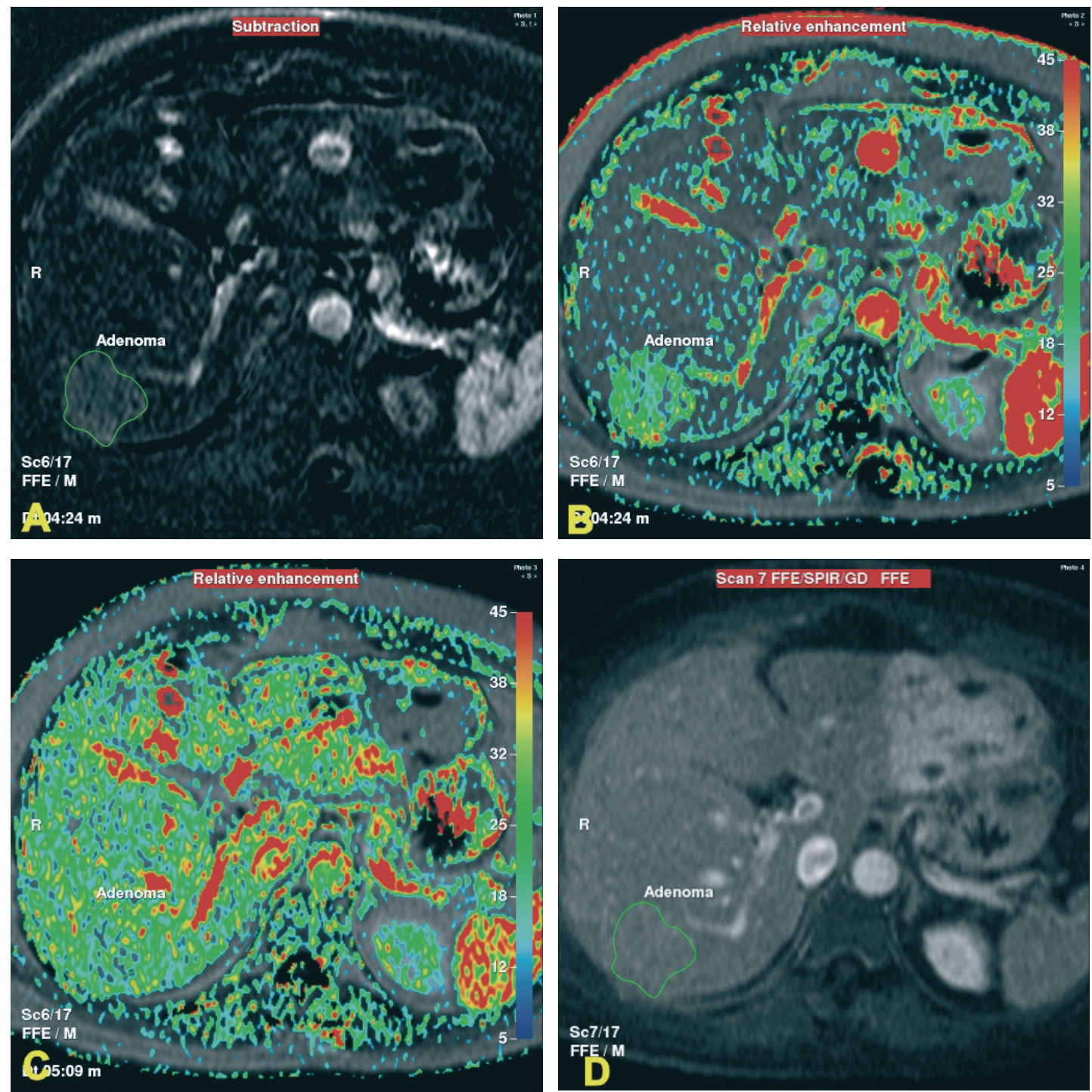

\section{Figure 4: Hepatocellular adenoma}

32-year-old female with a hepatocellular adenoma in the right lobe of liver and a hemangioma in the left lobe of the liver. A) (Subtraction image from the arterial phase) Hypervascular lesion. B) (Color-coded parameter image from the arterial phase, calculated using relative enhancement) Homogeneous blush of the lesion. C) (Color-coded parameter image from the portal phase, calculated using relative enhancement) Isointensity of the lesion with the surrounding liver. D) (Delayed fat-suppressed T1-weighted image) Isointensity of the adenoma in the right lobe of the liver and homogeneously enhanced hemangioma in the left lobe of the liver. Note: decremental value in color spectrum: red $>$ orange $>$ yellow $>$ green $>$ blue > indigo > violet. 

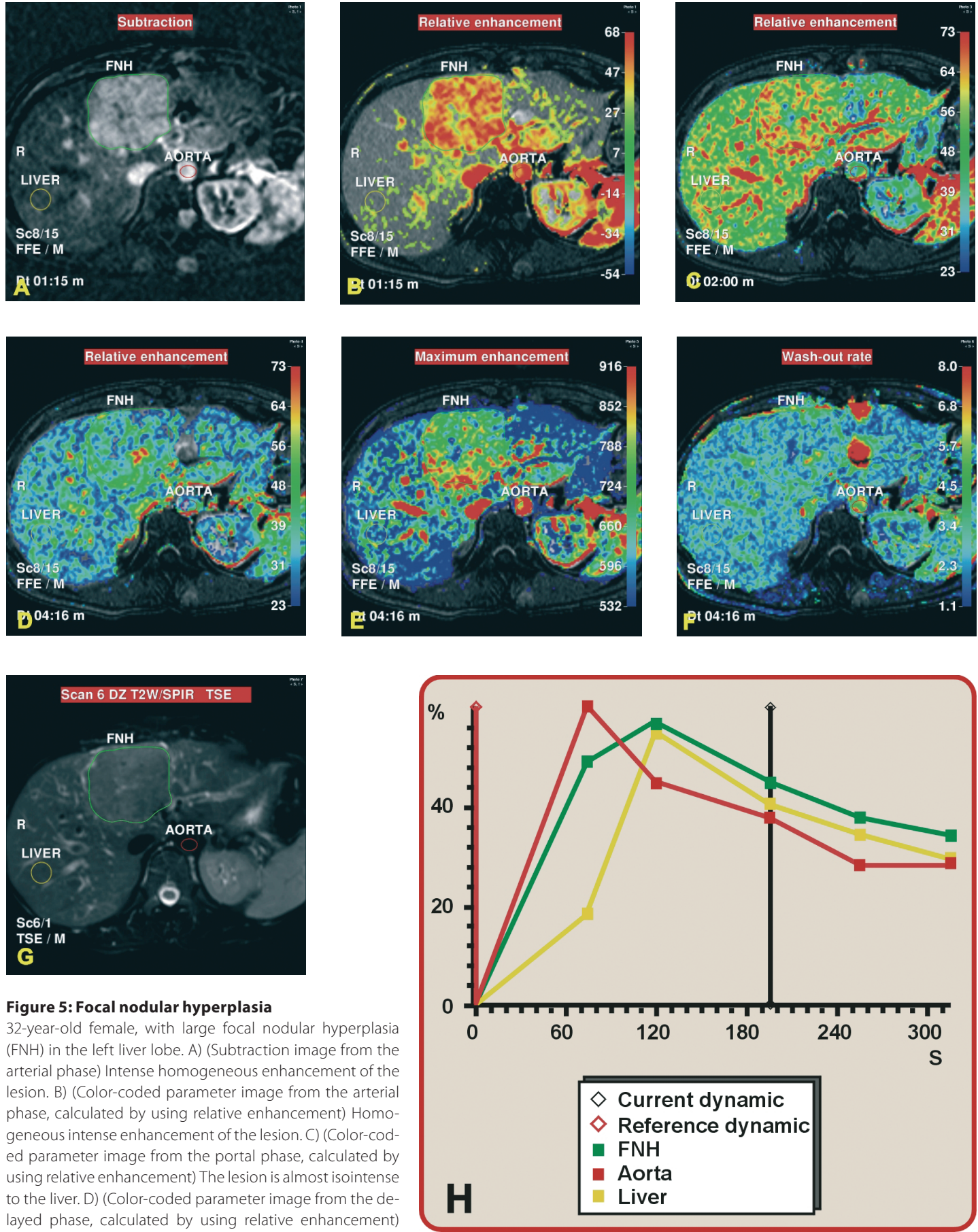

\section{Figure 5: Focal nodular hyperplasia}

32-year-old female, with large focal nodular hyperplasia (FNH) in the left liver lobe. A) (Subtraction image from the arterial phase) Intense homogeneous enhancement of the lesion. B) (Color-coded parameter image from the arterial phase, calculated by using relative enhancement) Homogeneous intense enhancement of the lesion. C) (Color-coded parameter image from the portal phase, calculated by using relative enhancement) The lesion is almost isointense to the liver. D) (Color-coded parameter image from the delayed phase, calculated by using relative enhancement) Delayed enhancement of the central scar. E) (Color-coded parameter image, calculated by using maximum enhancement) The absolute values of enhancement of the lesions are higher than the liver. F) (Color-coded parameter image, calculated by using wash-out rate) This lesion has a similar wash-out rate as the surrounding liver. G) (T2-weighted image (TR/TE=1500/80 msec) The lesion is only slightly hyperintense to the liver. H) The time-intensity curve of the lesion (green curve) parallels the curve of the aorta in the arterial phase, indicating a very steep enhancement (Type III). In the delayed phases it parallels the curve of the liver, reflecting its benignity as well as its similarity to the normal liver tissue. Note: decremental value in color spectrum: red $>$ orange $>$ yellow $>$ green $>$ blue $>$ indigo $>$ violet. 
CAHAPTHR
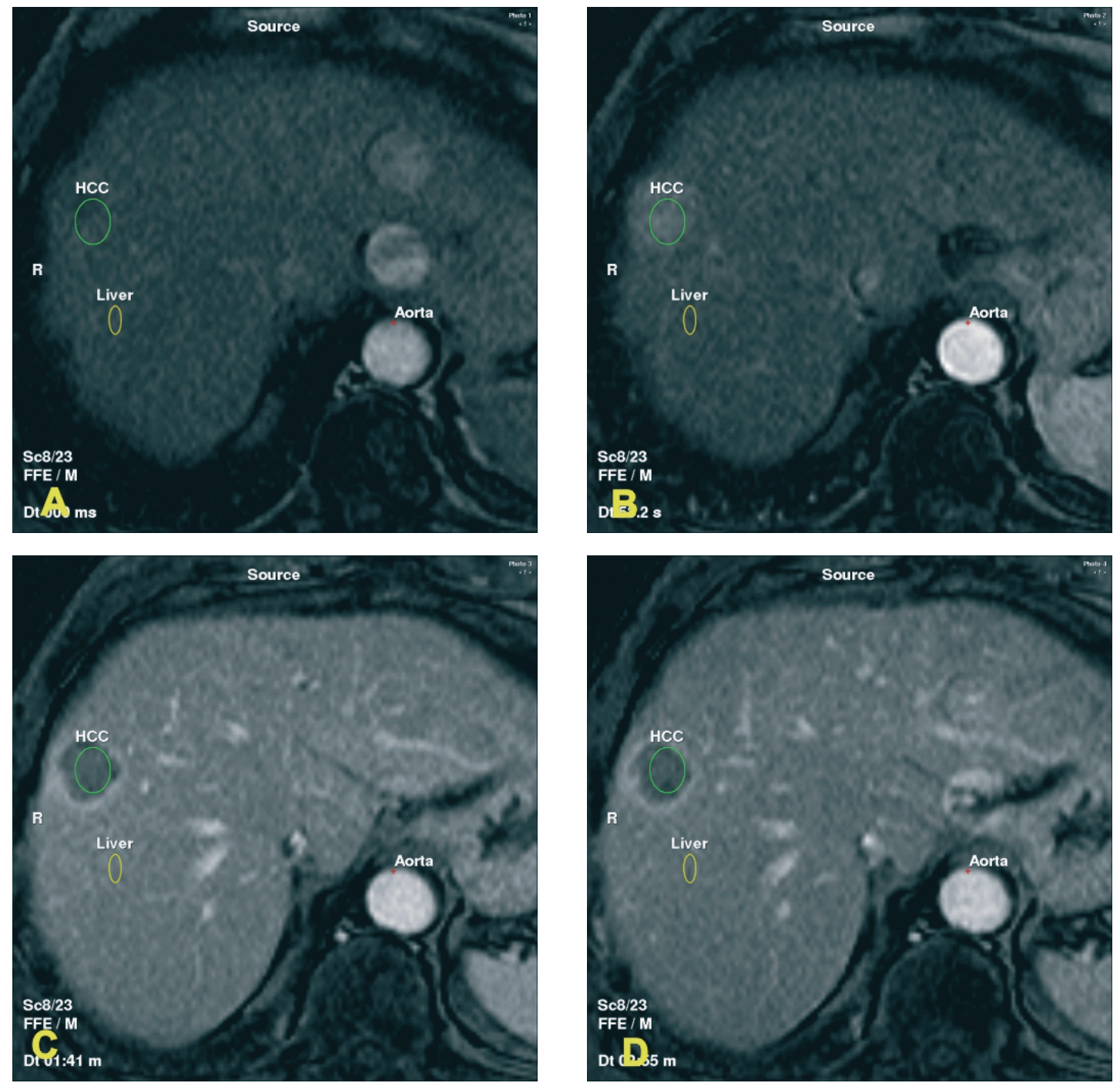

\section{Figure 6: Hepatocellular carcinoma}

62-year-old male with hepatocellular carcinoma (HCC) in the right liver lobe. A-D) Four images illustrating the typical four phases of dynamic gadolinium enhanced sequence, including the pre-contrast phase (A), arterial phase (B), portal phase (C), and delayed phase (D). Note that the HCC is intensely enhancing in the arterial phase and shows wash-out in the delayed phases with late enhancement of a pseudocapsule. 

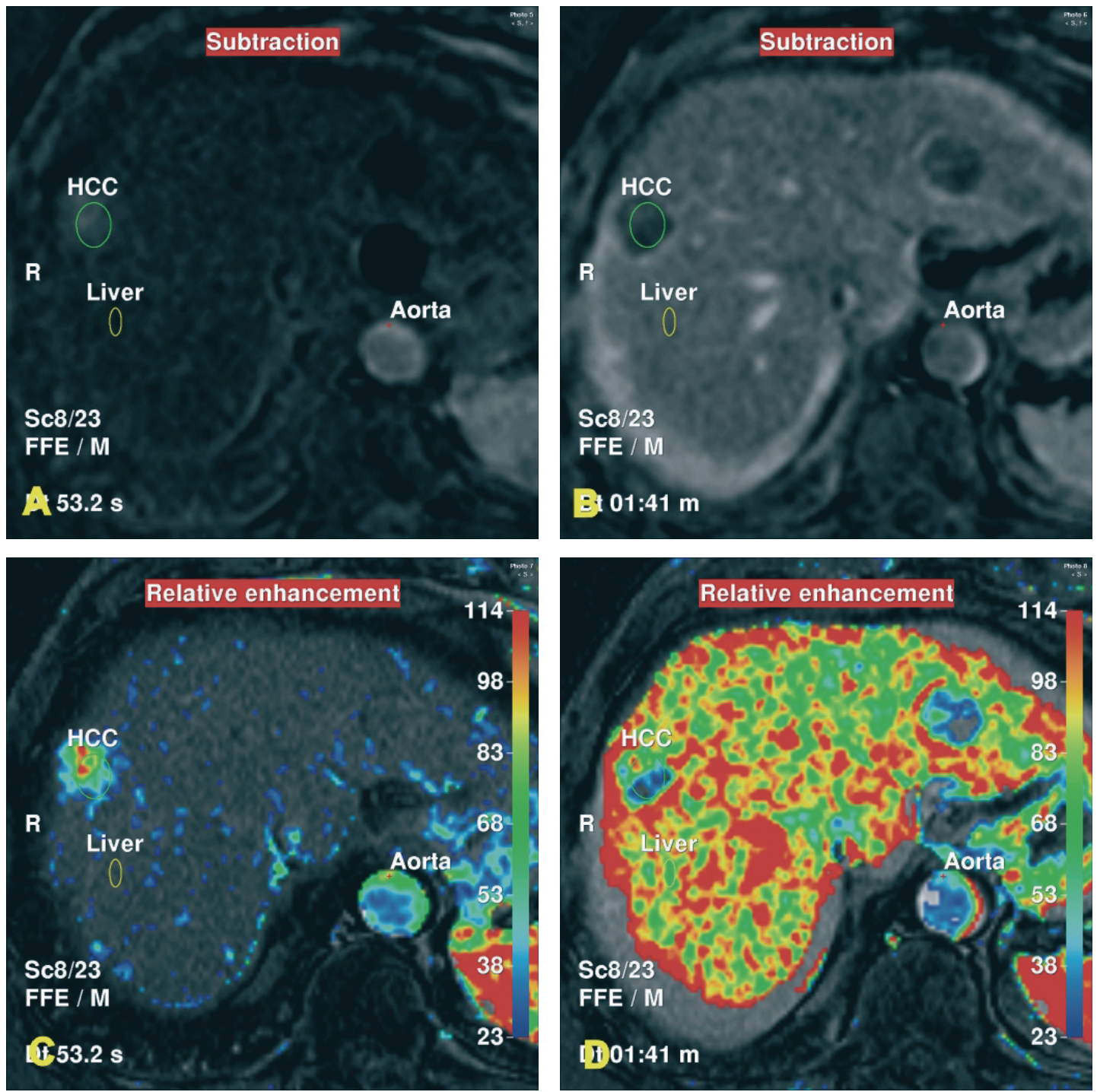

\section{Figure 7: Hepatocellular carcinoma}

The same patient as in Figure 6. A-B) (Two subtraction images from the arterial and portal phases, respectively) Improved visualization of the HCC. C-D) (Two color-coded parameter images from the arterial and portal phases, respectively, calculated by using relative enhancement) Heterogeneous enhancement of the lesion, i.e. the lesion is enhancing more intensely in the center than in the periphery (more red in the center) (C), and lost contrast in the center (sign of a high wash-out rate), with enhancing pseudocapsule (more red in the periphery of the lesion) (D). 

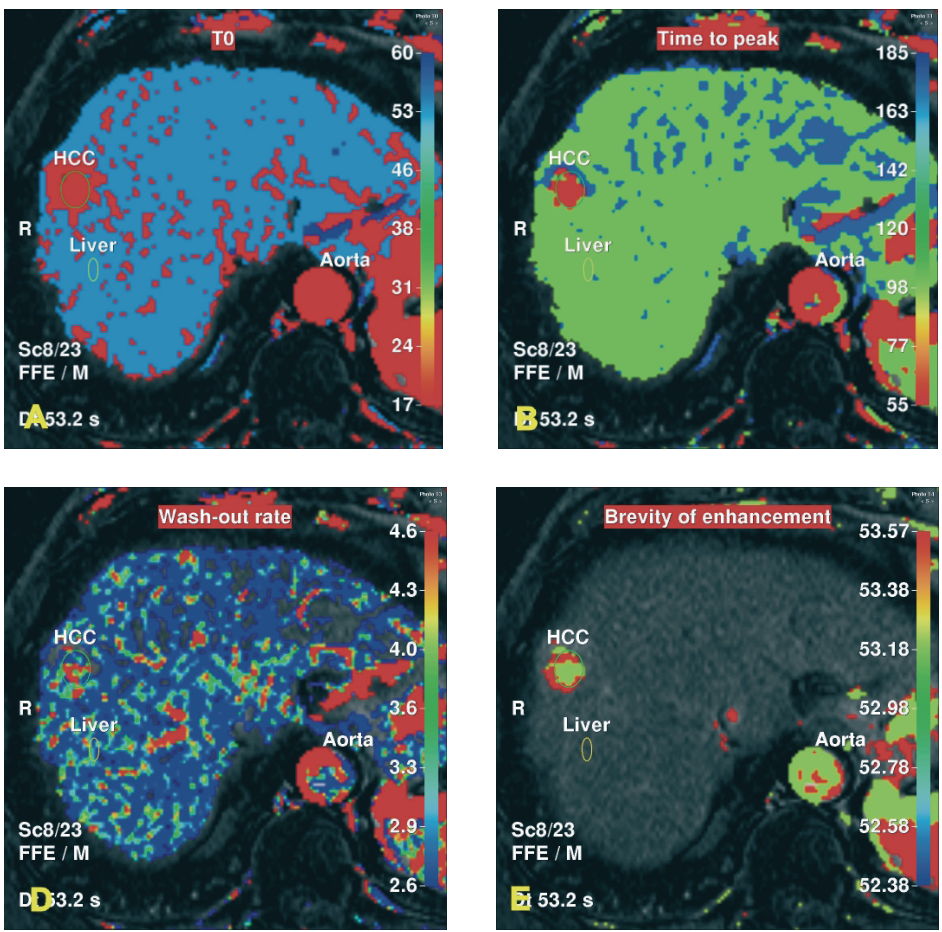
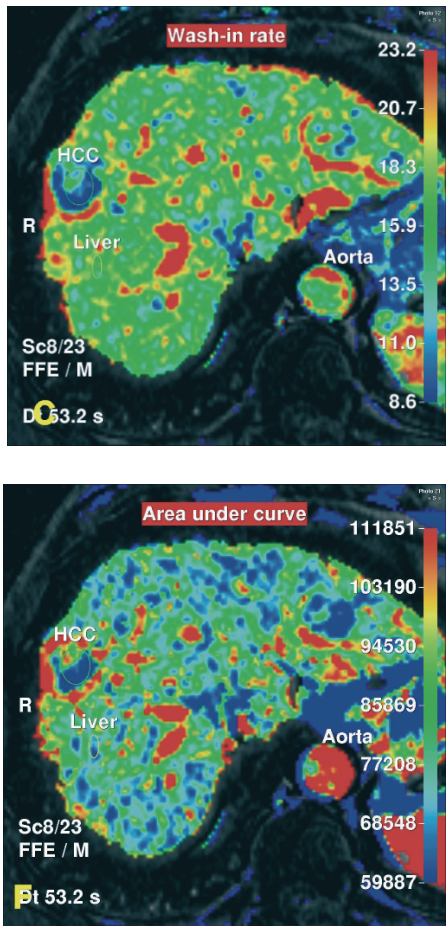

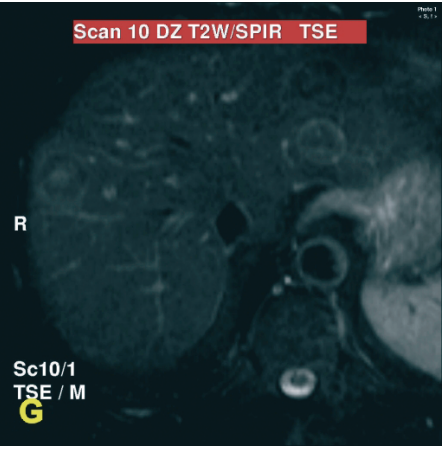

Figure 8: Hepatocellular carcinoma

The same patient as in Figures 6-7. This figure shows various parameters as color-coded images. A) (Color-coded parameter image, calculated by using T0) The lesion has a shorter T0 (i.e. enhances earlier) than the surrounding liver. B) (Color-coded parameter image, calculated by using time-to-peak (TTP)) The center of this lesion has a shorted TTP as compared to the pseudocapsule. This indicates that the peak of contrast reaches the center of the lesion in an earlier phase. C) (Color-coded parameter image, calculated by using wash-in rate) The center of this lesion has a lower wash-in rate as compared to the pseudocapsule. D) (Colorcoded parameter imaging, calculated by using wash-out rate) The center of the lesion has a slightly higher wash-out rate than the surrounding liver. E) (Color-coded parameter image, calculated by using brevity-of-enhancement) The time between the wash-in rate and wash-out rate is longer in the center of this lesion as compared to the surrounding liver. F) (Color-coded parameter image, calculated by using area-under-curve) The pseudocapsule of this lesion received a higher dosage of contrast agent than the center of the lesion. G) (T2- weighted image (TR/TE=1500/80 msec) Slightly hyperintensity in the center as well as the pseudocapsule of the lesion. H) The time-intensity curve shows that the lesion (green curve) enhances more than the liver and less than aorta in the arterial phase, and loses contrast in the later phases (sign of wash-out). Please note that the curve, unlike the curve in the FNH (see Figure $5 \mathrm{H}$ ), is not parallel to the curve of the liver in any phase. Note: decremental value in color spectrum: red $>$ orange $>$ yellow $>$ green $>$ blue $>$ indigo $>$ violet.

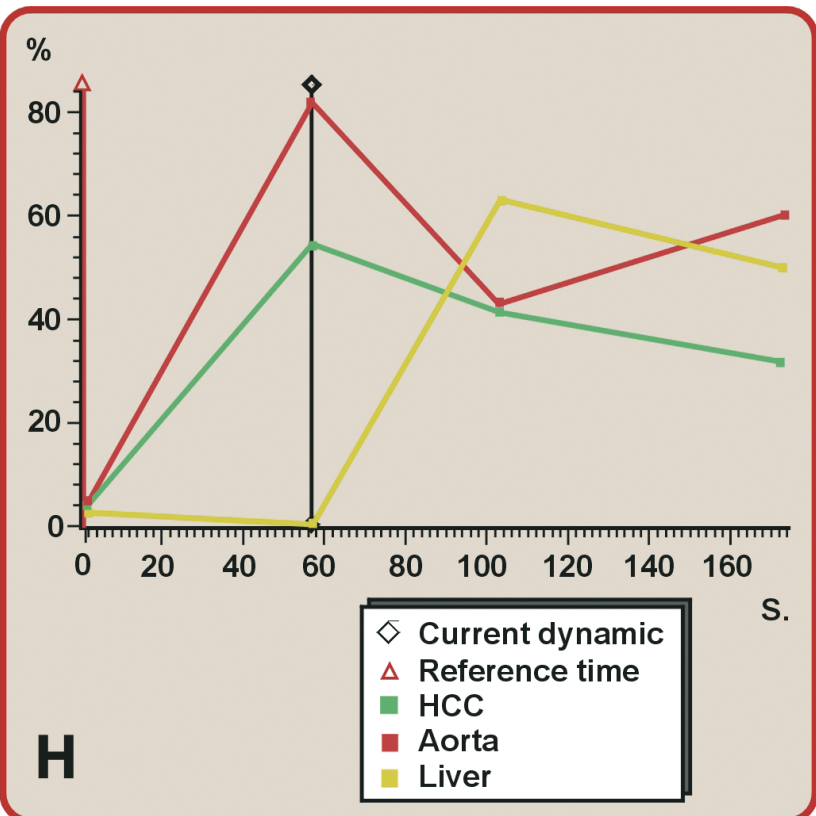



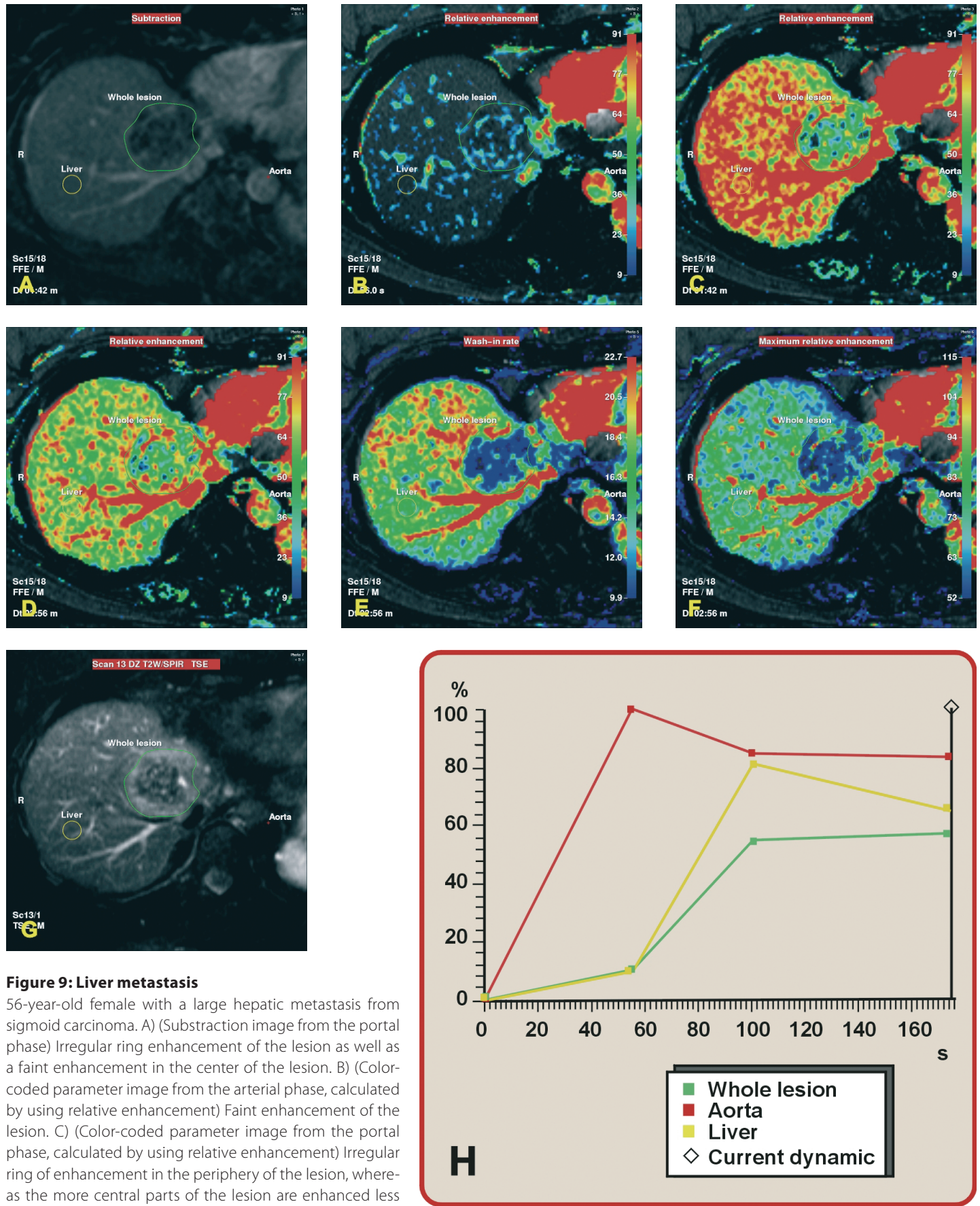

\section{Figure 9: Liver metastasis}

56-year-old female with a large hepatic metastasis from sigmoid carcinoma. A) (Substraction image from the portal phase) Irregular ring enhancement of the lesion as well as a faint enhancement in the center of the lesion. B) (Colorcoded parameter image from the arterial phase, calculated by using relative enhancement) Faint enhancement of the lesion. C) (Color-coded parameter image from the portal phase, calculated by using relative enhancement) Irregular ring of enhancement in the periphery of the lesion, whereas the more central parts of the lesion are enhanced less than the surrounding liver. D) (Color-coded parameter im-

age from the delayed phase, calculated by using relative enhancement) The lesion has enhanced slightly more than in the previous phase, whereas the liver has lost some of its contrast. Therefore, the conspicuity of lesion has decreased. E) (Color-coded parameter image, calculated by using wash-in rate) The periphery of the lesion has a higher wash-in rate than the center of the lesion. F) (Color-coded parameter image, calculated by using the maximum relative enhancement) The central parts of the lesion enhance less than the surrounding liver. G) (T2- weighted image (TR/ $T E=1500 / 80 \mathrm{msec}$ ) The periphery of the lesion is slightly hyperintense to the liver. H) The time-intensity curve of the lesion (green curve) shows that the lesion is enhancing similar to the liver in the arterial phase. In the portal phase, there is the greatest difference in enhancement between the lesion and the surrounding liver. In the delayed phase, the lesion shows slight further increase in enhancement, whereas, the liver loss some contrast. Therefore, the conspicuity of the lesion decreases after the portal phase. Note: decremental value in color spectrum: red > orange > yellow $>$ green $>$ blue $>$ indigo $>$ violet. 


\section{CARAPTHi}
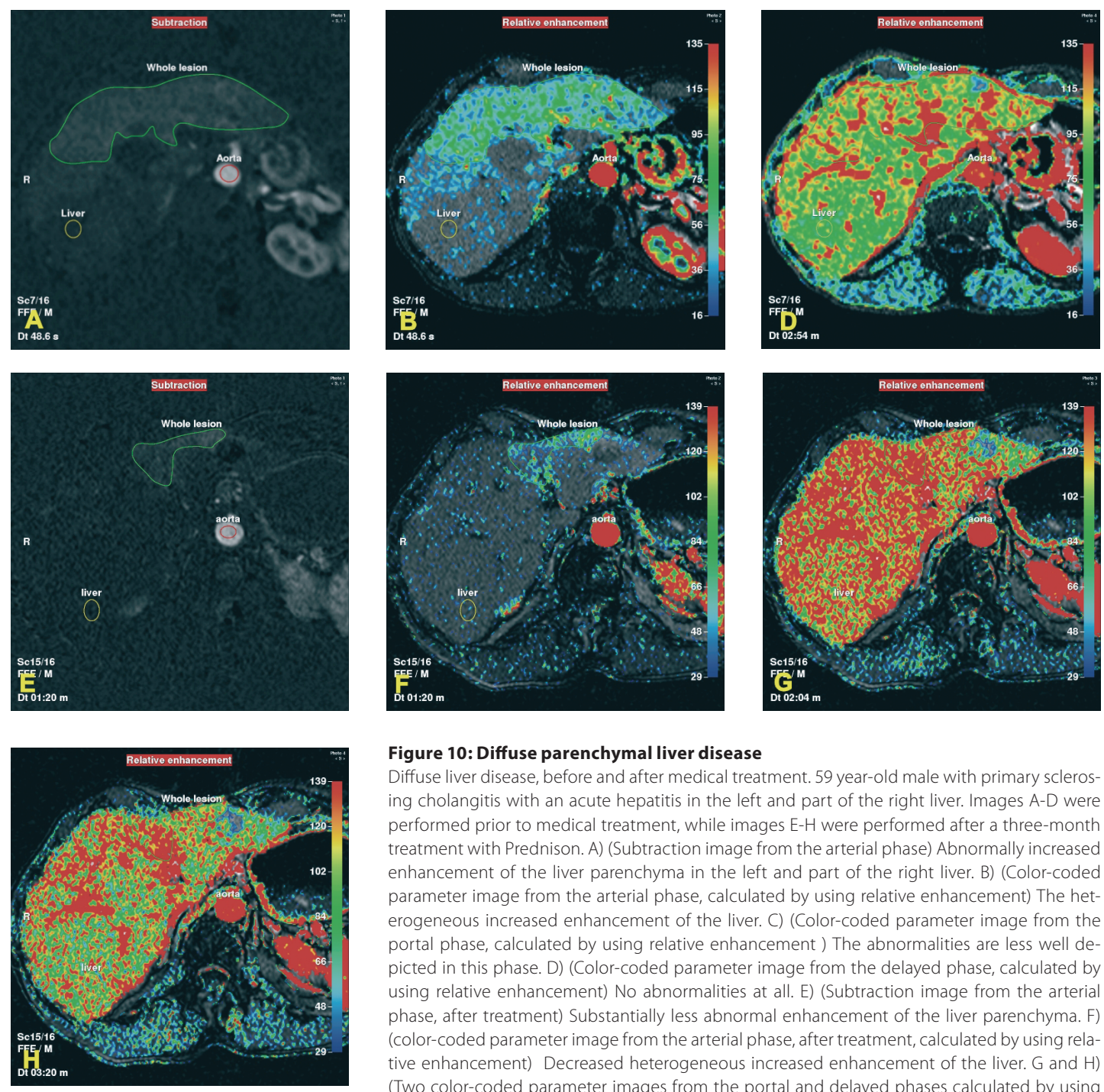

\section{Figure 10: Diffuse parenchymal liver disease}

Diffuse liver disease, before and after medical treatment. 59 year-old male with primary sclerosing cholangitis with an acute hepatitis in the left and part of the right liver. Images A-D were performed prior to medical treatment, while images $\mathrm{E}-\mathrm{H}$ were performed after a three-month treatment with Prednison. A) (Subtraction image from the arterial phase) Abnormally increased enhancement of the liver parenchyma in the left and part of the right liver. B) (Color-coded parameter image from the arterial phase, calculated by using relative enhancement) The heterogeneous increased enhancement of the liver. C) (Color-coded parameter image from the portal phase, calculated by using relative enhancement ) The abnormalities are less well depicted in this phase. D) (Color-coded parameter image from the delayed phase, calculated by using relative enhancement) No abnormalities at all. E) (Subtraction image from the arterial phase, after treatment) Substantially less abnormal enhancement of the liver parenchyma. F) (color-coded parameter image from the arterial phase, after treatment, calculated by using relative enhancement) Decreased heterogeneous increased enhancement of the liver. $\mathrm{G}$ and $\mathrm{H}$ ) (Two color-coded parameter images from the portal and delayed phases calculated by using relative enhancement) The abnormalities have become occult. Note: decremental value in color spectrum: red $>$ orange $>$ yellow $>$ green $>$ blue $>$ indigo $>$ violet. 